\title{
International EcoHealth One Health Congress 2016
}

\section{3}

\section{Economic Impact of Dengue in Rondonia State, Brazil, After Hydroelectric Plants Construction}

\section{Karina Abe and Simone Miraglia}

Universidade Federal de São Paulo

The Health Impact Assessment (HIA) has been developed in many countries and has its origin in Environmental Impact Assessment (EIA) studies. However, EIA did not comprehend fully health impacts derived from environmental aspects and HIA came to overcome it. The objective of this work was to analyze the association and economic impact of dengue cases near a hydroelectric construction area in Brazil. The methods presented a suggestive approach of HIA and included screening, scoping, appraisal of health impacts, economic valuation and recommendations. Rapid HIA was chosen and applied to dengue cases in Rondonia state, Brazil. Statistical analysis were performed by segmented regression followed by economic defensive expenditure method. The statistical analysis showed the construction of hydroelectric plants have contributed to increase the dengue cases in the state $(\mathrm{p} \leq 0.05)$ and the annual average cost has increased from US\$ 2,462,528 (after the construction) to US\$ 6,621,543. The hydroelectric plants in Brazil, despite having severe impacts on the environment and health of the population, were approved and released for construction before the impacts were properly assessed, including economic evaluation of health impacts. In this sense, the incorporation of HIA within the EIA process could be useful in the health management.

\section{Attention to the 'Bottom Billion', Their Animals and Shared Environment}

Bernadette Abela-Ridder ${ }^{1}$, Johannes Sommerfeld ${ }^{2}$ and Joss Kessels ${ }^{3}$

${ }^{1}$ Neglected Zoonotic Diseases, Department of the Control of Neglected Tropical Diseases, World Health Organization, Geneva, Switzerland

${ }^{2}$ Special Programme for Research and Training in Tropical Diseases, World Health Organisation, Geneva, Switzerland ${ }^{3}$ Joss Kessels, University of Queensland, Australia

The 'bottom billion' refers to the poorest and most marginalized people in the world today. These populations experience social and economic inequality, structural violence, harsh environmental conditions with often inadequate water supply and sanitation and close proximity to their animals. They lack access to basic preventative health measures, and experience higher incidences of diseases eliminated elsewhere. Preventable, endemic diseases are rarely prioritised for surveillance as they do not pose a risk of epidemic or pandemic outbreak. This is a failing on two levels: (1) the presence of preventable diseases acts as an indicator of the overall state of the health system; and (2) knowledge of 'usual' allows detection 
of the unusual. Strengthening surveillance and other systems for endemic diseases, infectious or otherwise, provides necessary infrastructure to both combat the existing and target the emerging.

This symposium aims at an interactive discussion on operationalising One Health in the context of neglected endemic diseases and strategies to work across sectors and platforms to maximise reach and impact. Our international panel will discuss perspectives on community engagement, disease surveillance, zoonotic risks, devastating animal bites, biodiversity loss, water and sanitation, with field examples on the control of specific issues as rabies, snake bites, cystic echinococcosis, among others.

679

\title{
SEACFMD Roadmap: A Framework to Control FMD in South-East Asia, China and Mongolia (2016-2020)
}

\section{Ronello Abila}

OIE South-East Asia

The regional initiative to control foot and mouth disease (FMD) in South-East started in 1994 when countries in the region agreed to set-up an OIE Sub-Commission for FMD control. In 1997, the members formally launched the South East-Asia FMD (SEAFMD) Campaign to coordinate a sub-regional programme to control the disease. In 2010, China joined the Campaign, thus it was renamed as South-East and China Foot and Mouth Disease (SEACFMD). In 2016, Mongolia joined the Campaign. To provide long-term guidance to the Campaign, a SEAFMD Roadmap 2020 was launched in 2007. Then in 2011, the 2nd edition was launched to give more emphasis at reducing the overall FMD prevalence by targeting hotspots and critical nodes along movement pathways, and progressive zoning approach in areas with the most advance stage of FMD control. Maintenance of FMD free countries and zones was also highlighted in the 2nd edition. In 2015, a 3rd edition of the Roadmap was developed to provide a framework to guide the Campaign from 2016 to 2020. The current SEACFMD roadmap continue a risk-based approach which identify areas where possible sources of FMD viruses are circulating, and focus the intervention in those areas.

\section{9}

\section{The Victorian Rabbit Action Network: Socio-Political-Ecological Insights from a Wicked Problem}

\author{
Lisa B. Adams ${ }^{1}$, Theodore R. Alter ${ }^{2}$, Margot W. Parkes ${ }^{3}$, Andrew P. Woolnough ${ }^{4}$, Michael Reid ${ }^{4}$ \\ ${ }^{1}$ Lisa Adams and Associates \\ ${ }^{2}$ The Pennsylvania State University \\ ${ }^{3}$ University of Northern British Columbia \\ ${ }^{4}$ Victorian Government Department of Economic Development, Jobs, Transport and Resources
}

The European rabbit was introduced into Australia for hunting in the 1880s. It has since colonized the continent and the hearts and minds of its people. Alongside these emotional attachments are the ecological and economic impacts; rabbits threaten over 300 vulnerable species and cost agribusinesses more than AUD\$200 million per year. These and other factors make rabbit management a pressing socio-ecological issue in Australia today. An ongoing challenge is to develop landscapescale management strategies that have broad community support. In 2013, the Invasive Animals Cooperative Research Centre initiated a research project to support community-led action for more sustainable and effective rabbit management in Victoria. The project led to the creation of the Victorian Rabbit Action Network (VRAN). We describe the emergence of VRAN to draw out insights on political economy and collective action. We invite those working with complex problems 
(such as invasive species and more generally), to consider the socio-political-ecological dimensions of these problems and the implications for professional practice.

\section{Global Health True Leaders: Internalizing the Core Life Values of True Leader in the Curriculum}

Wiku Adisasmito ${ }^{1,2}$, Vilda Rachman Amir $^{2}$, Ni Made Hermiyanti², Putu Mas Dewi Pratiwi², Alexandra Tatgyana Suatan ${ }^{2}$, Allan F Lauder ${ }^{3}$ and Marc Baril ${ }^{4}$

${ }^{1}$ Faculty of Public Health, Universitas Indonesia

${ }^{2}$ Indonesia One Health University Network

${ }^{3}$ Faculty of Humanities, Universitas Indonesia

${ }^{4}$ Stelerix Strategic Management, Inc., Canada

In the era of globalization with the borderless world, people and animal mobilization enables the countless threat of disease pathogen to spread across the world. As a threat faced by multisector, leading collaboration across sector is challenging. A true leader is needed to lead multisector collaboration to tackle disease threats. It motivated Universitas Indonesia and the Indonesia One Health University Network to start a program called Global Health True Leaders (GHTL) to nurture prospective one health workforce with core values of true leader in supporting multisector collaboration. The program is a combination of in-class training, field work, and leadership training which developed and integrated 10 core values on all activities. INDOHUN formed the values through leadership value mapping from national and international references. The values then summarized into 10 core values consist of 5 true leader characters (smart, responsible, brave, integrity, wise), and 5 true leader abilities (to think, to decide, to act, to communicate, and endurance). GHTL trainers will nurture these values through each module activities. This approach is expected to improve leadership skill among the participants to collaborate in combating one health problems.

967

\section{One Health Field Epidemiology Education and Training Program: FETP Revitalization in Indonesia}

Wiku Adisasmito ${ }^{1,2}$, Vilda Rachman Amir $^{2}$, Ni Made Hermiyanti ${ }^{2}$, Nurul Maretia Rahmayanti ${ }^{2}$, Putri Viona Sari ${ }^{2}$, Kaylee Myhre Errecaborde ${ }^{3}$ and Katey Pelican ${ }^{3}$

${ }^{1}$ Faculty of Public Health, Universitas Indonesia

${ }^{2}$ Indonesia One Health University Network

${ }^{3}$ Department of Veterinary Population Medicine, University of Minnesota

Threats from infectious diseases need to be addressed at the human-animal-environmental interface and require strong surveillance system that can support early detection and response. The application of field epidemiology skill is extremely relevant considering the risk of disease outbreaks in Indonesia. Indonesia needs 1250 field epidemiologists by 2020 , while it is estimated that current FETP will only able to prepare 420 field epidemiologists. FETP revitalization using multisector approach may needed to fulfill national demand and improve surveillance system. Two working groups that gather members from government, academia, and practitioners are established to address education and regulation issues. Working group for education focuses on curriculum standardization as well as the academic process. Working group for regulation advocates policy maker to protect the profession as well as approach them to utilize the workforce in central and local level. The members support FETP revitalization and promote the process among national stakeholders. 


\title{
Identifying Factors Associated with Rabies Mortality in Humans Through Verbal Autopsy
}

Be-Nazir Ahmed ${ }^{1}$, Umme Ruman Siddiqui ${ }^{2}$, Dhananjaya Karuna Ratna ${ }^{3}$, Sohel Rana ${ }^{4}$, Md Kamrul Islam ${ }^{4}$, Chandan Kumar Sarker ${ }^{4}$, Rashed Ali Shah ${ }^{5}$ and Aunta Melan ${ }^{6}$

${ }^{1} \mathrm{NIPSOM}$

${ }^{2} \mathrm{MO}$ CDC DGHS MHFW

${ }^{3}$ World Animal Protection

${ }^{4}$ CDC DGHS

${ }^{5} \mathrm{CDC}, \mathrm{DGHS}$

${ }^{6}$ Mymensingh Medical College

Bangladesh ranks third highest among rabies endemic countries. Causes of rabies often remain undetected. A verbal autopsy was conducted among 62 cases of rabies occurring from January to June, 2014 in Bangladesh to identify the associated factors. Majority (44\%) victims were of below years 15 and were male (66\%). The most responsible animal was dogs $(81 \%)$ that were mostly $(73 \%)$ stray with unknown vaccination status. The highest $(44 \%)$ injuries were in lower limbs and were of category III $(68 \%)$. Most of the victims (68\%) didn't receive post exposure prophylaxis but $70 \%$ of the victims did seek treatment from traditional healers. The incubation period ranged from 21 to 330 days with an average of 34 days. The characteristic manifestations were hydrophobia, photophobia and aerophobia. It is necessary to ensure better accessibility and availability of rabies post exposure prophylaxis in rural areas of Bangladesh.

\section{4}

\section{Addressing Antimicrobial Resistance Globally at the Human-Animal Inter- face Using a One Health Approach- World Health Organization (WHO) Initiatives}

\author{
Awa Aidara-Kane \\ World Health Organization (WHO)
}

WHO's work on the public Health impact of the use of antimicrobials in food producing animals is undertaken through different initiatives. WHO provide support to WHO Member countries to effectively respond to the increasing threat of antimicrobial resistance, with due consideration given to the importance of the food chain as a driver for emerging resistance patterns that threaten human health. In May 2015 WHO member states have adopted the Global Action Plan (GAP) to combat antimicrobial resistance emphasizing the need for a "One health" approach. With the support of the WHO Advisory Group on Integrated Surveillance of Antimicrobial Resistance (AGISAR) WHO developed a 5-year strategic plan to support implementation of the GAP at the human-animal interface. WHO activities include: (1) supporting the development and implementation of "One Health" national action plans to combat AMR; (2) Capacity building through dissemination of a WHO guidance document on integrated surveillance of AMR, training workshops and AGISAR country pilot projects promoting a multisectoral approach to AMR surveillance; (3) developing a global "Tricycle" AGISAR curriculum on integrated surveillance of ESBL Ecoli in humans, the food chain and the environment; and (4) developing recommendations to preserve the efficacy of critically important antimicrobials for human health. 


\title{
Microbial Source Tracking of Fecal Contaminants Using 16S rRNA Gene Sequencing and Phylogenetic Analysis
}

\author{
Fareeha Akhtar \\ University of Veterinary and Animal Sciences (UVAS) Lahore, and member of Pakistan biological safety association (life time \\ member)
}

The present study was designed for microbial source tracking of fecal contaminants using 16s rRNA gene sequencing and phylogenetic analysis. The targeted population in this experimental trial was that community of humans who lived in close proximity with animals with poor sanitary management system at ten different districts of Punjab Pakistan. The fecal, stool and droppings samples were collected from human and almost all types of animals and birds (either a pet or domesticated). Similarly drinking water samples and sewage samples were also collected from the same localities from where fecal samples were collected. The indicator organism in this study was E .coli. Phylogenetic analysis of $16 S \mathrm{rRNA}$ gene of the E. coli isolates recovered from feces of animals, poultry, human, sewage water and drinking water was performed. Comparison of 16S rRNA gene sequences of all the isolates by using Neighbor joining method with 1000 bootstrap value revealed that the $E$. coli isolate recovered from drinking water was sharing the same clade with the E. coli isolate from dog stool sample and sewage water sample. Similarly another sequence of E. coli isolate recovered from drinking water was found in the same clade with E. coli from horse dung. These results gave an indication of the possible contamination of drinking water with sewage water with more emphasis on the involvement of dog and horse fecal material. The results of this study revealed that the use of 16s rRNA gene sequencing and phylogenetic analysis can be a useful tool for source tracking the fecal contaminants of drinking water.

627

\section{Reported Zoonotic Diseases in the Sultanate of Oman, 2005-2015: Calling for One Health Surveillance System Approach}

Mohammed AL-Yaaqoubi and Michael Ward

University of Sydney

In this century the global healthcare system is facing many challenges, one notable example is zoonoses. The World Animal Health Database (WAHID) interface was reviewed for notification of zoonoses between 2005 and 2015 in the Sultanate of Oman. Only five zoonoses were reported: Brucellosis, Salmonellosis, Leptospirosis, Leishmeniasis and Crimean-Congo Hemorrhage Fever (CCHF). In general the number of human disease notifications for each were higher than in animals. Among these brucellosis was the most common disease notification in both humans and animals, whereas for CCHF (a cause of the highest mortality in humans) there were no confirmed notification in animals. Human brucellosis cases increased in number during the study period whereas reporting of animal cases fluctuated. Despite the reporting and prevalence of zoonoses apparently there is no integrated surveillance system for human and animal cases. The healthcare system in the Sultanate of Oman (i.e. Ministry of Health) includes a division for communicable diseases, however a similar division focusing on zoonoses is lacking. Development of such a surveillance system for animal health and the establishment of a One Health surveillance system for zoonotic diseases is recommended to address the impact of these diseases.

153

\section{Fixing Broken Food Systems}

Robyn Alders ${ }^{1}$, Mike Nunn ${ }^{2}$, Brigitte Bagnol ${ }^{3}$, Julian $\mathrm{Cribb}^{4}$, Richard Kock ${ }^{5}$ and Jonathan Rushton ${ }^{5}$ ${ }^{1}$ International Association for Ecology and Health 
${ }^{2}$ Australian Centre for International Agricultural Research

${ }^{3}$ University of Sydney

${ }^{4}$ Julian Cribb and Associates

${ }^{5}$ Royal Veterinary College

Defining and facilitating sustainable and ethical food systems that contribute to human and planetary health is amongst the greatest challenges facing our world today. Broken food systems have delivered the double burden of under- and over-nutrition, contributed to degradation of ecosystems, resulted in farming families becoming the working poor and perpetuate women's carrying the burden of health problems and poverty. By focusing on good nutrition and on nutrient cycles we can better understand and strengthen interrelationships between farmers, traders, regulators, consumers and policy-makers to determine policies and food systems that deliver appropriate, sustainable, diverse, ethical and nutritious diets nationally and globally.

To achieve sustainable food systems and adequately nourish 9 billion people by 2050, a paradigm shift is required and involves direct action from the soil level to the plate. We review options for improvements that will see sustainable, nutritious and safe food being produced and delivered with minimal waste. Such improvements will help consumers (re-) connect to the environment, with food gatherers and producers contributing to enhanced physical and mental human health and more resilient planetary health.

769

\title{
Coupled Dynamics of Water Quality, Childhood Diarrhea, and Wildlife Dynamics in a Dryland River System in Botswana
}

Kathleen A. Alexander, John Tyler Fox and Claire Sanderson

Virginia Tech

Water connects humans, animals, and microbial communities across diverse landscapes. Our work on water quality-health in the Chobe River region of Northern Botswana identifies important linkages between human-wildlife health and landscape dynamics. We quantified seasonal variation of Escherichia coli and Total Suspended Solids (TSS) in the Chobe River using spatiotemporal and geostatistical modeling of water quality time series data collected along a transect spanning a mosaic of protected, urban, and developing urban land use. We found significant relationships in dry season E. coli concentrations and protected land use, floodplain habitat, and fecal counts from elephant and other wildlife. Dry season fecal loading by both elephant and other wildlife were important predictors of early wet season $E$. coli concentrations. Outbreaks of diarrheal disease in children under five years of age (2006-2015) living in this region covaried significantly with water quality declines and the magnitude and timing of hydrological and meteorological factors. Multidrug resistance among E. coli isolated from wildlife in the protected areas up river of the urban center are similar to patients from the local hospital. Couplings between the environment, animals, and human health are complex and dynamic involving multiple interdependent drivers and feedback processes.

884

\section{Global Impact of Food Borne Diseases - A Case Study of a One Health Approach for Control of Taenia solium in Lao PDR}

\author{
John Allen ${ }^{1}$, Anna Okello ${ }^{2}$, Amanda Ash ${ }^{3}$, RC Andrew Thompson ${ }^{3}$, Phouth Inthavong ${ }^{4}$ and Boualam Kham- \\ lome ${ }^{5}$ \\ ${ }^{1}$ CSIRO Australian Animal Health Laboratory \\ ${ }^{2}$ CSIRO Australian Animal Health Laboratory and College of Medicine and Veterinary Medicine, University of Edinburgh \\ ${ }^{3}$ School of Veterinary and Life Sciences, Murdoch University \\ ${ }^{4}$ National Animal Health Laboratory, Ministry of Agriculture and Forestry
}


${ }^{5}$ Department of Communicable Disease Control, Ministry of Health

WHO publications characterise 31 food borne diseases comprising: 11 diarrhoeal agents; 7 invasive infectious agents; 10 helminths; and 3 chemical toxins. While the burden of foodborne disease is a global concern, WHO highlights African and South East Asia Regions with the highest incidence and death rates. Taenia solium taeniasis/cysticercosis is a Neglected Tropical Disease ranked first on the global scale of foodborne parasites and is of significant public health importance across Africa, Asia and Latin America. Humans are both the definitive and accidental dead-end host of the parasite, with consumption of undercooked pork, open defecation and free ranging pig production systems important risk factors for disease transmission. A coordinated approach by the human and animal health sectors in endemic countries is recommended for the effective control of taeniasis/cysticercosis. Yet to date, there has been little published on the outcomes of such coordinated approaches. Results of a coordinated intervention in a T. solium hyper-endemic village in Lao PDR are presented. Together with economic assessment of the cost effectiveness (abstract presented separately), this work provides evidence for policy makers to devise appropriate government intervention programs.

\title{
Using Problem Based Learning and Interactive Technology to Provide an Immersive Experiential Learning Environment in One Health for the Health Sciences Programs at Tufts University
}

\author{
Janetrix Hellen Amuguni ${ }^{1}$ and Melissa Mazan ${ }^{2}$ \\ ${ }^{1}$ Tufts University, Cummings school of Veterinary Medicne \\ ${ }^{2}$ Tufts University Cummings School of Veterinary Medicine
}

There is a critical need for students in veterinary, human and dental medicine to appreciate and develop expertise in One Health in order to promote and improve health for people, animals, and the environment. In this presentation, we describe an interdisciplinary web-based and manually available program developing and piloting One Health Problem Based Learning cases for use across the health sciences programs at Tufts University. In order to teach our students to practice effectively within the burgeoning field of One Health, we employ an approach that involves multidisciplinary collaborative efforts to combine the experience and knowledge base of practitioners across the Allied Health fields in order to develop intellectual resources within our students as well as teach valuable skills in teamwork and communication. Through funding from the Tufts Innovates Program a grant that supports multidisciplinary enquiry and collaboration, we assembled a cadre of faculty and students from across the schools to identify areas of thematic importance to create a One Health community of educators and learners. The outcome is six multi-disciplinary One Health PBL cases used across the health sciences schools. Facilitator and student OHPBL guides will be revised, completed and availed on line and manually.

\section{9}

\section{Mainstreaming Gender in One Health and Emerging Pandemic Threats Through a Regional Training of Trainers Program in East and Central Africa}

\author{
Janetrix Hellen Amuguni ${ }^{1}$, Anthony Mugisha ${ }^{2}$ and Niyati Shah ${ }^{3}$ \\ ${ }^{1}$ Tufts University, Cummings school of Veterinary Medicine \\ ${ }^{2}$ Makerere University College of Veterinary Medicine, Animal Resources and Biosecurity \\ ${ }^{3}$ United States Agency for International Development
}


The diverse roles played by men and women create different exposure mechanisms to emerging pandemic threats and infectious diseases. Gender roles, the distribution of labor, access and control over resources play an important part in the biosecurity, control, prevention and response to infectious diseases. Therefore gender differences need to be addressed to respond effectively to public health threats. One Health Central and Eastern Africa (OHCEA), a network of 17 schools of Public health and veterinary medicine in Africa has identified Gender as a key One Health core competency. With this in mind, OHCEA set out to ensure that gender as a strategic concept is included as an analytical and planning tool across programs by creating a Gender and One health training program. The training applies gender analysis tools to disease surveillance, response, prevention and control mechanisms, engenders One Health skills and competencies, identifies gender gaps and develops gender sensitive indicators and policies to address those gaps. Trainings target in service personnel in multiple disciplines, the private sector and university faculty and students from OHCEA institutions. The end goal is to create a gender integration training curriculum that weaves the key principles of gender equality and gender analysis throughout OHCEA institutions.

\title{
Complex Systems and Intensive Agriculture: 150 Years of Attempted Cropping in Northern Australia
}

\author{
Kate Andrews \\ Fenner School of Environment and Society, ANU
}

The persistent drive for nation building in Australia has revived the push to develop the great 'untapped' agricultural potential of northern Australia; framed by the need for international food security. The current Commonwealth Government has again allocated hundreds of millions of dollars for investment in the development of northern Australia. Yet over the last one hundred and fifty years failed attempts have accumulated; from ambitious Texan entrepreneurs losing millions of dollars to Australian families walking off small farms to Indigenous Australians being even further marginalised. Despite investing millions of dollars in supporting agricultural development in northern Australia the huge region has countered the international trend of agriculture intensification. Many factors contribute to this, including social and environmental. More importantly it is the relationship between these factors that inhibit the growth of intensive agriculture, and hinder our capacity to learn from experience. This paper explores those factors and our need to better understand complex systems in our agricultural and policy contexts.

907

\section{Impact of Integrated Approaches on the Reduction of Health and Environ- mental Risks Related to Water, Sanitation and Hygiene (WASH) in Disad- vantaged Urban Areas' of Abidjan (Côte d'Ivoire)}

\author{
Eliachie Larissa Emeline Angoua ${ }^{1,2,3}$, Kouassi Dongo ${ }^{1,2}$, Bassirou Bonfoh ${ }^{2}$ and Jakob Zinsstag ${ }^{3}$ \\ ${ }^{1}$ Université Félix Houphouët Boigny d'Abidjan (Côte d'ivoire) \\ ${ }^{2}$ Centre Suisse de Recherches Scientifiques en Côte d'Ivoire (CSRS) \\ ${ }^{3}$ Swiss Tropical and Public Health Institute (Swiss TPH)
}

Environmental and health risks related to WASH is still among the acute challenges facing by decision-makers and researchers. Improving WASH at the household level is therefore one key element in fighting human and environmental risk exposure. In Côte d'Ivoire, despite efforts to address the issue, particularly in slums, the situation fails from improvement. This study aims at reducing environmental and health risks related to WASH in urban slums using integrated approaches. The methodology combined field observations, surveys, and water bodies sampling. Firstly, we assessed the current status of sanitation in six selected slums. Secondly, we implemented integrated interventions (CLTS, Health 
education). Finally, the impact of the intervention will be assessed. The preliminary findings showed different social characteristics. But, in all the slums there is a lack of access to improved water (23\%), improved sanitation (57\%) and hygiene $(76 \%)$. This situation leads to environmental and water pollution, and causes health burdens (malaria, diarrhea...) among the population. The preliminary results allowed establishing relevant indicators to monitor and evaluate the impact of the interventions: (i) end of open defecation, (ii) end of illegal dumping of solid waste and wastewater in the streets, (iv) Hygiene practices around water sources and (v) latrines.

1022

\section{Using Climate and Weather Data to Predict and Mitigate Disease}

\section{Assaf Anyamba \\ United Space Research Association/NASA}

While not typically directly integrated into health resource allocation planning, climate and weather forecasting can provide important inputs for risk assessment and response for global and local health. Advances in satellite remote sensing enhance precision in risk prediction. In addition to increasingly-recognized utility for vector-borne disease (e.g. Rift Valley Fever virus) and extreme weather effects, this technology can also support action to reduce burden of non-communicable diseases (such as expected respiratory illness attributable to forest fires during periods of drought, or reduced food provision or supply due to flood events). Examples from 2015 to 2016 Global Climate Anomaly and Potential Disease Risks forecasting report will be presented, with consideration of additional data and systems that can support further improvement in risk prediction, as well as potential preventive measures that can be mobilized in partnership with other sectors for a more integrated understanding of the environmental determinants of health. Emerging technologies and data applications can enable us to anticipate and better mitigate and prepare for health threats.

175

\section{An Ecohealth Approach to Mitigating Antimicrobial Resistance: Antibiotic Use in Layer Farms in Central Java Province, Indonesia}

Riana Arief ${ }^{1}$, Ridvana Dwibawa Darmawan ${ }^{1}$, Sunandar ${ }^{1}$, Maria Digna Winda Widyastuti ${ }^{1}$, Erianto Nugroho ${ }^{1}$, Andri Jatikusumah ${ }^{1}$, Anak Agung Gde Putra, Edi Basuno ${ }^{2}$, Anis Karuniawati ${ }^{3}$, Hadri Latif, Agus Suwandono ${ }^{4}$, Iwan Willyanto and Imron Suandy

${ }^{1}$ Center for Indonesian Veterinary Analytical Studies

${ }^{2}$ The Center for Agriculture Socio-Economics and Policy Studies, Ministry of Agriculture

${ }^{3}$ Microbiology Department, Faculty of Medicine, University of Indonesia

${ }^{4}$ National Institute of Health, Research and Development, Ministry of Health

Imprudent use of antibiotics in both public and animal health fields raises the risk of antimicrobial resistance, a global health issue complicated by the human-animal interface. An ecohealth approach was applied to facilitate better understanding and develop potential intervention strategies. A cross-sectional survey of 40 layer farms in Karanganyar, Sukoharjo and Klaten districts in Central Java province, Indonesia, was conducted to collect baseline data on antibiotic use, accessibility, and farmer knowledge on antibiotic use and resistance. Majority of respondents were small scale farms with $\leq 5000$ birds $(62.5 \%)$ and had poor knowledge of antibiotics and resistance (53\%). Antibiotics were readily available at poultry shops $(60 \%)$ and technical services of pharmaceutical companies (53\%). Commonly used antibiotics were enrofloxacin $(60 \%)$, oxytetracycline $(38 \%)$, and brand combinations tetracycline-erythromycin $(38 \%)$ and oxytetracycline-neomycin (35\%), for both preventive and treatment purposes. Farms have self-combined different brands of antibiotics to treat difficult cases $(20 \%)$, a likely indication of antibiotic resistance. The presence of animal health services was weak in farms; 
only one had a veterinarian. However, $68 \%$ of respondents were open to advice from veterinarians and/or technical services. Based on these findings, intervention was focused on educating farmers and increasing access to veterinary services in farms.

649

\title{
An Ecohealth Approach to Mitigating Antimicrobial Resistance: Assessment of Doctor Kap (Knowledge, Attitude, Practice) And Programs in Health Facilities in Central Java Province, Indonesia
}

Winda Widyastuti ${ }^{1}$, Riana Arief ${ }^{1}$, Ridvana Dwibawa Darmawan ${ }^{1}$, Sunandar Sunandar ${ }^{1}$, Erianto Nugroho ${ }^{1}$, Andri Jatikususmah ${ }^{1}$, Hadri Latif ${ }^{2}$, Imron Suandy ${ }^{3}$, Edi Basuno ${ }^{4}$, Anak Agung Gde Putra ${ }^{5}$, Iwan Willyanto ${ }^{6}$, Agus Suwandono and Anis Karuniawati

${ }^{1}$ Center for Indonesian Veterinary Analytical Studies

${ }^{2}$ Animal Health and Veterinary Public Health Department, Faculty of Veterinary Medicine, Bogor Agricultural University, Bogor-Indonesia, Center for Indonesian Veterinary Analytical Studies

${ }^{3}$ Directorate of Veterinary Public Health, Directorate General of Livestock and Animal Health, Ministry of Agriculture, JakartaIndonesia

${ }^{4}$ Professional Consultant, The Center for Agriculture Socio-Economics and Policy Studies (ICASEPS), Ministry of Agriculture, Bogor-Indonesia

${ }^{5}$ Professional Consultant, Denpasar-Indonesia

${ }^{6}$ Professional Consultant, Surabaya-Indonesia

An ecosystem health approach was used to assess the capacity of health facilities and their medical staff in supporting antimicrobial resistance (AMR) control programs. Forty community health centers and 14 hospitals in Central Java, Indonesia were surveyed in 2014. Structured interviews were used to assess doctor knowledge, attitude and practices and the antibiotic provision system in the facility. Majority of doctors in community health centers (CHC) and hospitals had moderate $(77.5 \%)$ and high (71.4\%) levels of knowledge on antibiotics and drug resistance, respectively. All doctors expressed positive attitudes. In CHCs and hospitals, there was no patient education or surveillance program for AMR and doctors still prescribe antibiotics for non-specific diarrhea and acute respiratory infections. Antibiotic provision in CHCs is supervised by local Health Services. However, reporting of antibiotic use in private health facilities is not mandatory. This study recommends increasing doctor knowledge on antibiotics and AMR, establishing public education and information services on AMR in health facilities, initiating an AMR surveillance programs in hospitals and CHCs, and implement mandatory reporting of antibiotic use in private health facilities to local Health Services.

\section{Knowledge, Attitudes and Practices (Kap) Relating to Brucellosis in Small- holder Farmers in Pakistan}

\author{
Shumaila Arif ${ }^{1}$, Marta Hernandez-Jover ${ }^{1}$, Peter Thomson ${ }^{2}$, David McGill ${ }^{3}$ and Jane Heller ${ }^{1}$ \\ ${ }^{1}$ Charles Sturt University \\ ${ }^{2}$ The University of Sydney \\ ${ }^{3}$ The University of Melbourne
}

The aim of this study was to assess the extent of knowledge and understanding of brucellosis in smallholder dairy farmers in Pakistan and identify practices at the farm and household level that might pose a risk for humans contracting brucellosis. Between February and June 2015 a cross-sectional study was conducted among smallholder farms $(n=420)$ in the seven districts of Pakistan. Farmers were interviewed using a questionnaire to obtain information on farmers' knowledge about 
brucellosis and the potential risks for contracting brucellosis that are present for dairy farmers and their families. Logistic regression and ordinal logistic models were used to investigate potential predictors for risky behaviours. All farmers regularly performed at least one risky practice for brucellosis transmission from animal to human and animal to animal. From the multivariable model, it was demonstrated that the level of education and having heard about brucellosis were associated with prevalence of risky practices. In general, respondents with no formal education and those who had not heard of the disease displayed greater risky behaviour. Poor knowledge, wrong perception and attitude towards treatment, supports the need for including "one health" education in rural communities for any future control programmes in the country.

972

\section{Veterinary Aspects of Porcine Cysticercosis in Jayawijaya Regency Papua Province, Indonesia}

\section{Inriyanti Assa \\ Cenderawasih University}

Taenia solium cysticercosis is a significant public health problem in Papua, Indonesia. Porcine cysticercosis is most prevalent in areas of Jayawijaya with poor sanitation and traditional pig-keeping systems. The aim of this study was to determine veterinary aspects of porcine cysticercosis. The survey was carried out in eight districts of Jayawijaya between October 2009 and June 2011. A total of 104 pigs were tested serologically. Serum samples were tested for the presence of circulating parasite antigens using monoclonal antibody-based sandwich enzyme-linked immunosorbent assays (MoAb-ELISA). Forty samples (37.4\%) were positive by MoAb-ELISA; the highest prevalence was found in the district of Asolokobal (92.86\%) and the lowest prevalence (5.88\%) was in Wamena Kota. Pigs in the Asolokobal district were found to be highly vulnerable to porcine cysticercosis. The temperature of stones used to cook pork in a traditional manner is $300{ }^{\circ} \mathrm{C}$; cooking for 90 minutes with these stones results in an interior meat temperature of $60-90{ }^{\circ} \mathrm{C}$. It is therefore necessary to incorporate an anthropological approach to pig husbandry systems and pork cooking patterns into efforts to reduce porcine cysticercosis in Jayawijaya.

\section{A Health-Education Intervention to Reduce Cysticercosis/Taeniosis in Jayawijaya Regency, Papua}

\section{Inriyanti Assa \\ Cenderawasih University}

The purpose of this study was to assess the level of knowledge and behavior the community of Wamena, Jayawijaya Regency. The samples were housewives in Hubikosi $(\mathrm{n}=16)$ village, Yiwika $(\mathrm{n}=12)$, Milima $(\mathrm{n}=6)$ and Isaima $(\mathrm{n}=7)$. This research was health education experiment that divided by three phase; pre-intervention, post-intervention and one week after intervention, about knowledge and behavior of respondents, analyzed by two related sample Wilcoxon and paired sample t-test. The rate of knowledge during pre-intervention was $7.62 \%$. Post-intervention results obtained that knowledge about the causes and prevention of cysticercosis/taeniosis in terms of personal hygiene increase (87.37\%). Preintervention about behavior were washing hands after defecation (6.70\%) and duration pork cooking take more than an hour (95.83\%). Post-intervention, behavior of respondents were washing hands before meals (69.35\%), hand washing after defecation $(28.57 \%)$, defecation at latrine (13.99\%), boiling water $(50.30 \%)$, wash the pork before cooked $(20.16 \%)$, duration of burning stones more an hour (100\%) and eat pork contained cysts (91.67\%). One week after education, the knowledge (36.59\%) and behavior (21.39\%) increased. Health education continuously will reduce the prevalence of cysticercosis/taeniosis in Jayawijaya. 


\title{
Domestic Dogs as a Potential Bridge-Host Between Wild and Domestic/ Urban Habitats: OneHealth Implications
}

\author{
Francisca Astorga ${ }^{1}$, Daniela Poo-Muñoz ${ }^{2}$, Luis Emilio Escobar ${ }^{3}$ and Gonzalo Medina-Vogel ${ }^{4}$ \\ ${ }^{1}$ Pulso Ambiental/Amevefas \\ ${ }^{2}$ Escuela de Medicina Veterinaria, Universidad Santo Tomás Sede Temuco \\ ${ }^{3}$ Minnesota Aquatic Invasive Species Research Center and Department of Veterinary Population Medicine, University of \\ Minnesota \\ ${ }^{4}$ Facultad de Ecología y Recursos Naturales, Universidad Andres Bello
}

Domestic dogs, humans' best friend, are an emerging problem in many developing countries. Free ranging dogs, which may invade a wide range of natural areas, have an impact on wildlife (e.g., predation, competition, disease transmission) and a role in many zoonotic diseases. Dogs may be a subsidized bridge between wild and urban environments, or between wild and domestic cycles of diseases. In this study, we explored the potential role of dogs as bridges between bat-borne rabies and humans in Chile, which reports only a few canine-borne cases of rabies in the last decades, and maintains an endemic wildcycle in bats. For this, we developed a spatial analysis, using ecological niche models of bat-reservoirs, dog density, and the reported cases of dog rabies in the last 30 years. Previous studies have determined that the threshold for dog-rabies maintenance is $4.5 \mathrm{dogs} / \mathrm{km}^{2}$. Based on this, we developed a risk map for bat-borne rabies in dogs at a national scale, and evaluated it considering previous cases. Our risk-map revealed that the spatial approach is a useful tool for targeting strategies plans to control bat-borne rabies in Chile. Dog overpopulation needs a multidisciplinary approach, as it involves social, environmental, ecological and public health elements.

803

\section{Clinically Relevant ESBL-Producing Enterobacteriaceae in Wild and Domestic Birds: Evidence of Wide-Scale Environmental Dissemination of Antibiotic Resistance in Bangladesh?}

\section{Hasan Badrul \\ Department of Medical Sciences, Uppsala University}

The environmental dissemination of members of the Enterobacteriaceae family with ESBLs and MBLs has become a global concern. To explore the environmental contamination of antibiotic resistance in Bangladesh, and of ESBLs and MBLs in particular, fecal samples from poultry and wild birds were studied. Fecal droppings wild ducks and, poultry ( $\mathrm{n}=96$ ), gull $(\mathrm{n}=150)$, Open bill stork $(\mathrm{n}=170)$, crows $(\mathrm{n}=238)$ and Pigeon $(\mathrm{n}=150)$ were collected from house hold, coasts, lakes and hospitals grounds. ESBL producing Isolates were identified and characterized by culture, antibiotic susceptibility, PCR, sequencing and MLST. Plasmid transferability of ESBL-producers was assayed by conjugation experiments. Our results showed that ESBL and their multi-resistance in bacteria from different birds varied among bird populations-mostly based on their lifestyle and feeding behavior. No NDM-producer found among these birds, but ESBL-producers could be found in up to $59 \%$ of the crows, the birds with the highest carriage rate of multiresistant Enterobacteriaceae of all bird species studied. The most common ESBL-type was CTX-M-15, which also common in human population in Bangladesh. Birds carried clinically important sequence types including E. coli clone O25b-ST131. ESBL-producing E. coli had transferable plasmids. The high level of antibiotic resistant and ESBL-producing bacteria in the bird population of Bangladesh is worrying, and there is no easy solution in sight. 


\section{Investigation of Brucella spp Isolates of Humans and Livestock in Mongolia Using Multi Locus Variable Number of Tandem Repeat Analysis (MLVA-16)}

Zolzaya Baljinnyam ${ }^{1}$, Batbaatar Vanaabaatar ${ }^{2}$, Ulziisaikhan Gombosuren ${ }^{2}$, Enkhtuul Batchuluun ${ }^{2}$, Khurtsbaatar Ochirbat $^{2}$, Erdenebaatar Janchivdorj ${ }^{2}$, Selenge Tsend ${ }^{3}$, Esther Schelling ${ }^{4}$, Paola Pilo ${ }^{5}$ and Jakob Zinsstag ${ }^{4}$

${ }^{1}$ Animal Health Project, Swiss Agency for Development and Cooperation SDC, Ulaaanbaatar, Mongolia

${ }^{2}$ Institute of Veterinary Medicine, Mongolian University of Life Science, Ulaanbaatar, Mongolia

${ }^{3}$ National Centre for Communicable Diseases, Ulaanbaatar, Mongolia

${ }^{4}$ Swiss Tropical and Public Health Institute, University of Basel, Switzerland

${ }^{5}$ Institute for Veterinary Bacteriology, University of Bern, Switzerland

Brucellosis is one of the most common zoonotic diseases found worldwide. In Mongolia, human brucellosis first became an issue during the 1960s and since 1975, a mass vaccination strategy of small ruminants and cattle has successfully reduced human brucellosis cases from 48 cases (1974 year) to 0.23 case per 100,000 population at the end of the 1985. After 1990, human brucellosis re-emerged due to the severe decline in medical and veterinary services and lack of economic resources during the post-communist transition period. By 2003, Mongolia was ranked the second highest in terms of human brucellosis cases worldwide. It is therefore surprising, that despite the high prevalence of brucellosis, there has been hardly any genetic characterization of brucellosis in the country. In this study we characterized 58 isolates of B. melitensis and B. abortus from humans and livestock within five provinces of Mongolia using a 16 Multi Locus Variable number tandem repeat Analysis (MLVA-16). The 58 Mongolian strains were genetically more diverse when compared to Central Asian strains. Human strains were most closely associated to B. melitensis strains from sheep and goats. To the best of our knowledge, this is the first report on MLVA-16 characterized Brucella spp. strains from Mongolia.

\section{Forest-Based Livelihoods, Deforestation and Malaria in Southeast Asia: The Relevance of the Chronotone for Understanding Malaria Persistence at the Forest Fringe}

Melanie Bannister-Tyrrell ${ }^{1}$, Annette Erhart ${ }^{2}$, Nguyen Xuan $\mathrm{Xa}^{3}$, Lies Durnez ${ }^{1}$, Vincent Sluydts ${ }^{1}$, Charlotte Gryseels ${ }^{1}$, Sochantha Tho ${ }^{4}$, Marc Coosemans ${ }^{1}$, Po Ly ${ }^{4}$, Nicola James ${ }^{5}$, Anna Rosanas-Urgell ${ }^{1}$, Shunmay Yeung ${ }^{5}$ and Koen Peeters Grietens

${ }^{1}$ Institute of Tropical Medicine, Antwerp

${ }^{2}$ Medical Research Council, The Gambia

${ }^{3}$ National Institute of Malariology, Parasitology and Entomology, Vietnam

${ }^{4}$ National Center for Parasitology Entomology and Malaria Control, Cambodia

${ }^{5}$ London School of Hygiene and Tropical Medicine

Deforestation in Southeast Asia has coincided with a substantial decline in malaria across the region, principally because local vectors require shaded forest environments. Malaria transmission is now limited to remote forested regions, especially border areas, where the forest is a focus of legal and illegal economic activities. Often these areas are inhabited by ethnic minorities, who are traditionally dependent on slash and burn agriculture, requiring overnight stays at their farms located in the forest. In these remaining malaria hotspots, 'forest-going' is recognized as the major risk factor for malaria infection, however how 'forest-going' manifests as a risk behavior in the context of deforestation becomes more complex. Using a case study approach, based on extensive mixed-methods field research in three settings in Vietnam and Cambodia, the concept of the chronotone will be employed to demonstrate that transitions in livelihoods as the forest recedes can contribute to local 
malaria hotspots persisting, even as malaria incidence in the region declines. It will be argued that persistent malaria transmission is driven by shifting access to forest resources, and that the exposure risks associated with 'forest-going' are best understood at a social network rather than individual level.

873

\title{
The "Mosquito Police" Initiative in Northern Haiti: Engaging Communities in Larval Source Management for Integrated Control of Aedes, Culex and Anopheles Vectors
}

\begin{abstract}
Kevin Bardosh
Despite the growing consensus that current mosquito control tools are inadequate, surprisingly few large-scale programs deploy community-based larval surveillance and control. In Latin America and the Caribbean, Zika virus has challenged us to develop more robust social mobilization techniques. This paper will discuss the design and implementation of a demonstration project in Northern Haiti that aims to enact major policy changes in how "top-down" vector control teams engage communities for the integrated control of Zika, dengue, Chikungunya, malaria and lymphatic filariasis. Our approach (currently implemented in 8 villages, $>2,000$ households) is based on a stepwise process of community engagement orientated around a grassroots network of "Mosquito Police" (Polisy Kont Moustik, in Creole). This network conducts weekly larval surveillance and mobilizes their communities for a series of didactic behavior change activities, including environmental management, household modifications and community-wide visual arts competitions. This presentation will discuss how local culture, social norms, political dynamics and environmental conditions influenced our intervention and the scale-up process. We will situate this research within the broader global context of integrated vector management (IVM), with specific reference to new elimination goals for malaria and the international Zika response.
\end{abstract}

659

\section{Using an EcoHealth Approach to Understand the Smallholder Pig Production System in San Simon, Pampanga, Philippines}

Tamsin Barnes ${ }^{1}$, Paul John J. Alvaran ${ }^{2}$, Tessa Lyrene D. C. Lantican ${ }^{2}$, Eduardo L. Lapuz ${ }^{2}$, Augusto S. Baluyut ${ }^{3}$, Christopher R. Parke ${ }^{1}$, Gomathy Palaniappan ${ }^{1}$, Don Cameron ${ }^{1}$, Sherri Maigne A. Meneses ${ }^{4}$, Rico C. Ancog ${ }^{5}$, Ronnie Domingo ${ }^{6}$, Milagros R. Mananggit, Conny Turni, Joanne Meers, Chiara Palmieri, John I. Alawneh, Ronilo de Castro, Edwin C. Villar and Patrick J. Blackall

${ }^{1}$ The University of Queensland, Gatton, Australia

${ }^{2}$ Department of Agriculture 3-RADDL, Pampanga, Philippines

${ }^{3}$ Provincial Veterinary Office of Pampanga, City of San Fernando, Pampanga, Philippines

${ }^{4}$ Livestock Research Division PCAARRD-DOST, Los Baños, Laguna, Philippines

${ }^{5}$ University of the Philippines, Los Baños, Philippines

${ }^{6}$ Bureau of Animal Industries, Quezon City, Philippines

Smallholders are important contributors to pork production in the Philippines. A project aiming to improve the competitiveness of the smallholder pig system using an EcoHealth approach is underway in Pampanga, Philippines. A baseline survey of smallholder pig raisers was conducted, followed by farm visits, semi-structured interviews and focus groups with farmers and other stakeholders. Several management issues were identified. Water provision to sows was often restricted; some farmers believed that free access to water could drown piglets in utero/cause diarrhoea in piglets. The diet of sows during gestation was often inadequate in protein and energy. Other problems reported included diarrhoea, ill thrift and crushing in piglets. These findings were discussed with a focus group of farmers. Several interventions were proposed: 
seminars on water/nutrition and piglet management with input from successful farmers, a workshop to develop a sustainable record keeping system for smallholders and laboratory analyses of diarrhoea samples and drinking water. These steps will be the action component of the first of a series of cycles. Reflection will be used for knowledge generation in the next cycle. The strong participatory component of this research gives ownership to farmers and should help to improve their pig production and thereby their livelihoods.

\title{
AfyaData, a Mobile and Web Application Pair for Community Level One Health Security
}

Eric Beda Mutagahywa ${ }^{1}$, Esron Karimuribo ${ }^{2}$, Calvin Sindato ${ }^{3}$, Mpoki Mwabukusi $^{1}$, Godluck Akyoo ${ }^{1}$, Renfrid Ngolongolo ${ }^{1}$ and Mark Rweyemamu

${ }^{1}$ Southern African Centre for Infectious Disease Surveillance

${ }^{2}$ Sokoine University of Agriculture

${ }^{3}$ Tanzania National Institute for Medical Research

We present AfyaData, A novel one-health community centric surveillance system comprised by a mobile and web application pair. The goal of the system is to provide tools that foster one health security at community level, by empowering community health workers (CHW) in (a) active disease surveillance; by using AfyaData mobile tool for reporting symptoms and clinical signs. (b) Respond to health events within their community; by receiving immediate feedback on advisable course of action in response to submitted data; and (c) equipping health experts with community submitted health events, charting and mapping tools and ability to directly interact with CHW. This completes the cycle of data submission, expert advice and feedback. At the centre of AfyaData is a One Health Knowledge Repository and intelligent analytical scoring subsystem that matches and grades submitted symptoms and clinical signs to a set of pre-configured clinical case definitions and expert authored health content. Various thresholds based on location, occurrences may be configured to trigger alerts. The surveillance system developed is linked with national human and animal health disease surveillance and response systems so that capacity for early detection and response to disease events occurring at community level are managed in near to real time.

1051

\section{Rethinking Tuberculosis: Understanding Disease Progression and Consequences}

\author{
Brianna Beechler ${ }^{1}$, Anna Jolles ${ }^{2}$, Vanessa Ezenwa ${ }^{3}$ and Kate Boersma ${ }^{4}$ \\ ${ }^{1}$ Oregon State University College of Veterinary Medicine \\ ${ }^{2}$ Oregon State University \\ ${ }^{3}$ University of Georgia \\ ${ }^{4}$ University of San Diego
}

Tuberculosis (TB), caused by Mycobacterium tuberculosis and M. bovis, is notoriously hard to prevent reliably, eradicate fully from human and animal populations and even to treat in individual hosts, especially since the advent of MDR and XDR TB. Despite massive investment in its control in human and animal populations, the World Health Organization reports there were still 1.5 million people killed by TB in 2015 and 9.6 million cases were reported. We followed 317 freeliving buffalo in Kruger National Park for 4 years to monitor bovine tuberculosis incidence and progression. We have previously demonstrated that buffalo suffer health consequences, including declines in condition and increased risk of mortality-but that this risk can be prevented with the removal of gastrointestinal helminths. In this presentation we will 
also demonstrate that TB dramatically changes the host's microbial infra-community, increasing the prevalence and diversity of co-infecting parasites and pathogens and restructuring the animals' microbiomes. The information we have gleaned from this longitudinal study of African buffalo is pertinent not only to understanding the spread of BTB in bovids (buffalo and cattle), but also as a model for understanding control strategies in humans.

874

\title{
Using the Global Green and Healthy Hospital Network to Create a Healthier World
}

Carol Behne

The health sector is a significant contributor to greenhouse gas emissions and ecological harm. Both of these are risk factors for human health, despite the health sector's mission to protect and promote health. As one of the biggest industries in the world, the health sector can play a leading role in responding to climate change and reducing its ecological impact. The Global Green and Healthy Hospitals Network is a worldwide community working together to reduce their environmental footprint, and move to healthier, more sustainable low carbon operations. Launched in 2012, the network is driving transformative changes in the health care sector with over 20,000 members worldwide. The network is a virtual global community, supported by innovative technology, with members working together to chart their progress, share best practices and find solutions to common challenges. Nurses, facilities managers, executives, and sustainability professionals are all part of spearheading initiatives across ten goal areas - leadership, energy, waste, water, buildings, chemicals, food, transport, pharmaceuticals and purchasing. With members in every continent, Global Green and Healthy Hospitals is demonstrating the health care sector can lead a transformation in sustainable operations through collaboration, and a commitment to finding shared solutions to global and local challenges.

1039

\section{What Interest of Microscopy in Diagnosis of Plasmodium Falciparum for Better Management of Malaria in Current Context?}

\author{
Sylvain Beourou ${ }^{1}$, Alphonsine Kouassi $^{2}$, andre toure ${ }^{3}$, Issiaka Bassinka ${ }^{4}$, Louis Penali ${ }^{5}$ and Ronan Jambou ${ }^{6}$ \\ ${ }^{1}$ Pasteur Institute of Côte d'Ivoire \\ ${ }^{2}$ Institut Pasteur de Côte d'Ivoire
}

Microscopy remains WHO gold standard. It is performed in hospitals as a standard method. Through the external quality assessment programs (EQA) in microbiology conducted. One third of the regional health centers are still performing it. The purpose being to control the microscopy diagnosis quality and technicians performance. Thick blood smears, thin blood film, stained slides were performed by the National Reference Center (NRC) for malaria chemoresistance housed in IPCI. A questionnaire was submitted to participants. Including slide code, clinical information about the patient, the result of thick blood smear with parasite density, the result of thin blood film with identification of species and sexual forms and results interpretation. On a total of twenty-four (24) smears stained slides and notwithstanding the parasitemia, only $30 \%$ of correct answers were recorded for P. falciparum identification and none for other species. Parasitemia was approximate. Microscopy identification of parasite being WHO standard method entails a major problem. It requires a high qualified staff which is not available in areas where malaria is endemic. Unfortunately rapid tests are not satisfactory as well. New tools like PCR strip should be evaluated to replace microscopy for a strategic fight against malaria. 


\title{
New Communication Technologies: Connecting People and Data for Public Health Emergencies
}

\author{
Theresa Bernardo \\ University of Guelph
}

The 2010 Haitian earthquake represented a turning point in the use of digital technologies (cell phones, social media, open software) by virtual volunteers (programmers, GIS experts, translators, project managers) to augment the efforts of official responders. Since then, the Digital Humanitarian Network has grown to include tens of thousands of volunteers. With each new emergency, they have improved their skills and abilities under a variety of circumstances. They have incorporated new tools and capabilities, such as drones and big data analytics, as they became available. Although initially met with skepticism, virtual volunteers have proven their worth and their methods are being adopted by traditional responders. Rather than waiting for first responders to arrive, or adding to the operational burden by increasing the number of persons on site, it makes sense to do as much as possible remotely. Advances in artificial intelligence are improving our ability to analyze big data in real time and simple methods, like text and voice, are being developed to interact with big data. Barriers such as place and language are being overcome. Cheaper, faster and ubiquitous technologies will aid in the future response to public health emergencies, but can also be used to prevent them.

\section{4}

\section{Involve Me And I Understand: Using Experiential Learning In A Community One Health "Startup"}

Amanda Berrian ${ }^{1}$, Martin Smith ${ }^{1}$, Jacques van Rooyen ${ }^{2}$, Beatriz Martinez Lopez ${ }^{1}$, Woutrina Smith ${ }^{1}$ and Patricia Conrad $^{1}$

${ }^{1}$ University of California, Davis, USA

${ }^{2}$ University of Pretoria, South Africa

In the Mpumalanga Province of South Africa, bordering the Great Limpopo Transfrontier Conservation Area, agro-pastoralist communities reside and are characterized by a high degree of poverty and chronic infections, including HIV. To begin to address the health challenges at this human-domestic animal-wildlife interface, we developed an innovative One Health Training and Leadership program that utilizes a comprehensive biosecurity curriculum to teach the concepts of pathogen transmission, risk assessment, and risk mitigation. Local facilitators who receive pedagogical and leadership training implement the curriculum to promote community participation and sustainability. The curriculum uses an experiential learning model and has participants conduct an integrated One Health risk assessment of their household and environment. Participants photo-document high-risk interfaces to facilitate the development of an individual, actionable risk mitigation plan. In its first implementation, nearly $90 \%$ (69/78) of participants completed the multi-week training. Mean scores on pre/post written assessments for facilitators and participant cohorts improved by $17 \%(\mathrm{p}=0.0015)$ and up to $9 \%(\mathrm{p}=0.0509)$, respectively. Qualitative analytics revealed the early adoption of learned concepts, including improved hygiene practices and enhanced livestock housing. The program's model is scalable, allowing for adaptation and implementation in resource-poor, high-risk communities around the world.

\section{4}

\section{Estimating Public Burden Of Rabies In Ethiopia}

Tariku Jibat Beyene, Monique C.M. Mourits ${ }^{1}$, Abraham Haile ${ }^{2}$ and Henk Hogeveen ${ }^{1}$

${ }^{1}$ Wageningen University

${ }^{2}$ Ethiopian Public Health Institute 
The current public health burden of rabies in Ethiopia is quantified by assessing the cost of illness based on data obtained from extensive animal bite case survey, reflecting the human rabies exposure during the period September 2013 to August 2014. The cost quantification accounted for direct and indirect medical costs related to the treatment of humans being bitten by rabid suspected dogs, as well as the disability adjusted life years (DALY) resulting from human rabid cases. Animal bite victims were traced using data collected from recorded cases at health centres as well as by information obtained from questioning the local community to trace unregistered cases. Annually 135, 101 and 86 potential rabid dog exposures were estimated per 100,000 inhabitants in urban, rural highland and rural lowland districts respectively. The average monetary loss per corresponding district was estimated to be 23,25.6 and 32.7 USD per bite case while indirect cost in terms of DALY equalled 19.4, 136.7 and 82.4 DALY/100,000 population/year. This study demonstrated that rabies has a substantial health burden and economic impact comparable to the top 20 diseases causing premature deaths in Ethiopia. This calls for urgent one health approach action and implementation of safe and effective control measures.

688

\title{
Maintaining Dog-Mediated Rabies Elimination in Sikkim, India
}

\author{
Thinlay Bhutia ${ }^{1}$, Diki Palmu Sherpa ${ }^{2}$, Helen Byrnes ${ }^{3}$ and Andrea Britton ${ }^{3}$ \\ ${ }^{1}$ Sikkiim Anti-Rabies and Animal Health Division \& Program \\ ${ }^{2}$ SARAH Program \\ ${ }^{3}$ Vets Beyond Borders
}

The Sikkim Anti-Rabies and Animal Health program (SARAH) is a state-wide rabies program successfully eliminating dogmediated human rabies for over ten years. Maintaining freedom from dog-mediated rabies has been challenging with distemper outbreaks diminishing the vaccinated dog population and socioeconomic developments on the West Bengal borders of Sikkim. Two rabies incursions have been investigated using a One Health approach with Chief Medical Officers and Department of Forestry Officers in southern Sikkim notifying the SARAH Division of suspect rabies in people, dogs and cattle. The two outbreaks investigated occurred in early January 2015 and 2016, the first involving a jackal biting two people and a dog, and the second report involving an unvaccinated imported dog biting its owner who subsequently died five weeks later with symptoms of rabies. Laboratory confirmed diagnosis of human rabies is difficult due to community resistance and remote location. Rapid antigen test is used on suspect animal rabid cases but further laboratory diagnosis and molecular testing is needed. Rapid response teams were sent to the area to conduct tracing of in-contact animals and mass vaccination of dogs, cats and some livestock in a ten kilometre radius. An active surveillance program is being implemented and legislation strengthened for pre-border control.

\section{6}

\section{The Challenges of Curriculum Development for One Health Education: Insights From a Survey in Southeast Asia}

\author{
Flavie Luce Goutard ${ }^{1}$, Sabine Didierlaurent ${ }^{2}$, Francois Roger ${ }^{1}$, Aurelie Binot ${ }^{1}$ and Mathilde Paul ${ }^{3}$ \\ ${ }^{1}$ UR AGIRs, CIRAD \\ ${ }^{2} U R$ AGIRs, CIRAD/DGAL \\ ${ }^{3} I N P-E N V T$
}

Integrating $\mathrm{OH}$ approaches into education programs is now recognized as a way to cut across the silos of disciplines. The goal of this study was to provide an overview of the status quo of academic teaching activities in $\mathrm{OH}$ in Southeast Asia in 2015, and identify possible room for improvement under the new $\mathrm{OH}$ program InterRisk. Courses related to $\mathrm{OH}$ or Ecohealth concepts were identified, and contacts were taken with people involved into these programs to implement in depth interviews. 
Questionnaires were structured in 3 sections addressing (i) pedagogic objectives (ii) modules' structure and (iii) funding scheme. Twelve educational programs were identified in Thailand, Vietnam, Indonesia and Cambodia, and 21 interviews were conducted. The majorities of the courses $(8 / 12)$ are very broadly open to different disciplinary branches, relating to health, ecological or environmental sciences. Only the ones of short duration are attracting diverse disciplines. Environmental modules are well represented, while economic and social sciences are present only in half of the programs. This study provides a first overview of $\mathrm{OH}$ academic training in Southeast Asia. It can bring ways of improvement about the need for teachers' training, the promotion of interdisciplinarity and a better integration of social sciences in education.

901

\title{
Addressing Food Security Within a One Health Approach: Integration of Health and Agriculture Issues in the Socio-Ecosystem's Dynamics
}

\author{
Aurelie Binot \\ CIRAD-AGIRS, Campus International de Baillarguet
}

In a global context of increasing urbanization and high population densities, growing intensification of trade and farming systems, drastic land use changes and biodiversity erosion, food security is directly related to complex health issues emerging at the Animal-Human-Environmental interface. Indeed, global changes affecting biodiversity, water management and agricultural production may unexpectedly increase the risk of zoonotic and environmental diseases transmission, threatening food security, with devastating socioeconomic and wellbeing impacts for the poorest communities. Assessing and managing these risks implies taking into account socioecological dynamics, in link with epidemiological patterns and public-health policies. For instance, epidemiological surveillance of animal diseases has to involve economic costs and social impacts associated to disease reporting for the farmers. As zoonotic diseases' spreading can be largely induced by important ecological modifications, it implies to deeply understand eco-epidemiological systems involving pathogens and their reservoir, vectors and hosts. It requests a holistic approach to understand the relationship between "Health" and "Agriculture" within the socioecosystem's dynamics. It implies to underline the institutional constraints and potential coordination gaps across sectors and institutions and across action levels. Such integrative approach at the interface between ecosystems and societies cannot be achieved spontaneously, and implies methodological guidelines for cross-sectorial and interdisciplinary collaborations.

974

\section{A Collaborative Framework for Advancing Pathogen Detection at the Livestock - Wildlife Interface}

\author{
Peter Black, Chintana Chanthavisouk
}

FAO

A collaborative framework for pathogen detection at the livestock - wildlife interface is being implemented in a number of countries in Asia and Africa as a key component of the USAID funded Emerging Pandemic Threats Phase 2 (EPT-2) Programme. This framework contributes to the broader goal of determining the risk of pathogen spillover at human, livestock and wildlife interfaces. The framework aims to collect a common core set of samples from livestock species (e.g. poultry, pigs and cattle) - at the same times and at the same locations - that wildlife samples are collected. Staff from PREDICT arrange and perform the wildlife sampling while the United Nations Food and Agriculture Organization (FAO) arranges the livestock sampling. FAO has close relationships in country with the counterpart Departments of Agriculture and it is this relationship which underpins the capacity for livestock sampling within this collaborative framework. This synchronized surveillance requires good collaboration and coordination between officers from FAO, Departments of Agriculture and PREDICT. Site selection, data collection, animal sampling, testing and information management system issues will be described using the experience of implementing the framework in Lao PDR during 2016 as an example. 
1021

\title{
Strategic Foresight for Sustainable Agriculture for People, Animals and Environment
}

\section{Peter Black}

Food and Agriculture Organization of the UN

Strategic foresight - the process of organizational planning to anticipate future uncertainties - can guide more futureoriented planning around risks as well as opportunities. Between now and 2050, it is estimated that agricultural production will need to increase by $70 \%$ to meet projected demand. In this context, it is vital to reconcile and form synergies around often seemingly siloed priorities including food and nutrition security, animal and human health, biosecurity, and economic development - all while under increasing pressure from climate and other environmental change. Strategic foresight approaches lead to the conclusion that sustainability is about maintaining the complex systems that support our longerterm survival and health. When this is realised, the most fundamental drivers that underpin sustainable agriculture for people, animals and environment can be more readily recognized and reconciled. However, this process is extremely challenging as it does require a fundamental shift in our thinking and a close examination of what it is that societies value. Key research questions and challenges from the food and agriculture sector will be raised to stimulate thinking and discussion on areas of collaboration in animal, human and environmental health.

\section{4}

\section{Towards One Health? Embedding 'Social Licence to Operate' in Stakeholder Engagement for Mosquito Population Control}

Erin Bohensky, Samantha Stone-Jovicich, Lucy Carter and James Butler CSIRO Land and Water

With mosquito-borne diseases such as Zika and dengue on the rise, there are unprecedented investments in novel technologies for reducing wild mosquito populations that transmit disease. However, advancing these technologies comprises complex social, ethical, legal, and environmental considerations that span multiple disciplines and partnerships, i.e. a One Health approach. In this paper we explore the potential of the 'social licence to operate' (SLO) concept in technology-centred control of mosquito-borne diseases. SLO, widely used in the mining industry, refers to the informal acceptance, approval or trust a local community extends to a corporate entity or industry sector. We discuss our use of a SLO approach in partnership with Australian communities to assess the acceptability of releasing sterile male mosquitoes for dengue control. We present the approach, developed through an iterative process of integrating findings of previous SLO studies, justice approaches in ethics, and adaptive collaborative management, and engagement with community members and other stakeholders. We discuss the potential and limitations of using such an approach to guide meaningful stakeholder engagement and technological practices that reflect the social expectations and values of communities; increase trust and goodwill; and enhance relationships between private industry, scientists and communities.

\section{Building Capacity in One Health Research to Fill Health System Gaps in Africa: Supply and Demand of Approaches}

\author{
Bassirou Bonfoh, Katharina Heitz-Tokpa and Gilbert Fokou \\ Afrique One-ASPIRE/CSRS
}

Human and animal populations in sub-Saharan Africa live in a highly dynamic socio-ecological context that maintains endemic and favours emerging zoonotic diseases. Their transmission is complex, making it difficult to apply classical 
biological risk-based intervention approaches. The recent Ebola outbreak in West Africa has shown serious gaps in the public health system and the urgent need for a joint human and animal diseases surveillance-response system. In such environments, how do we introduce the One Health thinking at different scales of the health system? Transdisciplinary capacity in One Health thinking has the merit of achieving high health intervention impact during crisis, but it needs to be sustained with comprehensive socio-economic and cultural incentives. Building on the achievements of the consortium Afrique One, the new «African Science Partnership for Intervention Research Excellence » programme aspires to bring together leading institutions in One Health to develop research capacity and training for the next generation of scientists and practitioners facing ecosystem health, disease control and potential elimination challenges. This contribution shares success stories and challenges of how research-based knowledge of One Health can be transferred to different stakeholder groups in order to address common health problems and resilience patterns in the global South.

467

\section{Implications for Surveillance in Wild Birds and Poultry Following Recent Highly Pathogenic Avian Influenza Activity in Europe}

Andrew Breed, Adam Brouwer, Mark Arnold, Pablo Alarcon, Guus Koch, Ruth Bouwstra and Ian Brown Animal and Plant Health Agency

During 2014-2016 changes to patterns in detections of Highly Pathogenic Avian Influenza (HPAI) in Europe showed altered epidemiology affecting the performance of surveillance programmes. Incursion of a new lineage of H5 HPAI (clade 2.3.4.4) from East Asia caused multiple outbreaks in various poultry species and wild birds across six countries with an apparent reduction in mortality rates in domestic and wild waterfowl. This impacts the role and efficiency of scanning surveillance in poultry and surveillance of wild birds found dead. Significant antigenic variation was also observed on serological tests for this lineage of virus affecting test performance. Haemagglutination inhibition testing of sera from ducks with standard EU-recommended antigens suggested much lower sensitivity than when the homologous antigen was used. Results of a Bayesian analysis of these data will be presented. A separate outbreak of European lineage H5 virus in France affected over 90 farms with multiple Neuraminidase subtype combinations detected (H5N1, H5N2, H5N3 and H5N9). Infections were detected most often in domestic ducks, often in the absence of clinical signs of infection. The increasing availability and power of molecular epidemiology and genotyping has potential to rapidly inform on likely transmission pathways and identify strains or mutations of particular concern.

\section{Between-Roost Contact and Population Size are Essential for Maintenance of European Bat Lyssavirus Type 2 Infection in Myotis daubentonii: 'The Swarming Hypothesis'}

Andrew Breed, Daniel Horton, Mark Arnold, Graham Smith, James Aegerter, Lorraine McElhinney, Nick Johnson, Ash Banyard, Paul Racey and Tony Fooks

Animal and Plant Health Agency

Understanding the dynamics of zoonotic pathogens in their reservoir hosts is crucial to inform spill-over risk, yet our understanding of these dynamics is frequently insufficient. A particular challenge is presented by viral infections in bats as host-pathogen interactions may differ from other mammals. We investigated viral persistence in a wild bat population by combining empirical data and in silico analyses to test hypotheses concerning methods of viral persistence. This study investigates a fatal zoonotic virus, European Bat Lyssavirus type 2 (EBLV-2), in the Daubenton's bat. A total of 1800 bats were sampled for evidence for antibody and virus excretion during a nine year serial cross-sectional survey. Multivariate 
statistical models demonstrated age-related differences in seroprevalence, with significant variation in seropositivity over time and between roosts. An approximate Bayesian computation approach was used to model the infection dynamics, under multiple scenarios incorporating the known host ecology. These results demonstrate that EBLV-2 is endemic in the study population, and suggest that seasonal mixing between roosts during swarming events is necessary to maintain EBLV-2 in the population. These findings contribute to understanding how bat viruses can persist despite low prevalence of infection, and why infections may be constrained to certain bat species in multispecies roosts and ecosystems.

\section{Exploring Animal Rabies Endemicity to Inform Human Control Programs: A Case Study in the Punjab, India}

Victoria Brookes $^{1}$, G. S. Gill ${ }^{2}$, C. K. Singh ${ }^{2}$, B. S. Sandhu ${ }^{2}$, Navneet Dhand ${ }^{1}$, B. B. Singh ${ }^{2}$, J. P. S. Gill ${ }^{2}$ and Michael P. Ward ${ }^{1}$

${ }^{1}$ Faculty of Veterinary Science, School of Life and Environmental Sciences, The University of Sydney, NSW, Australia

${ }^{2}$ Guru Angad Dev Veterinary and Animal Sciences University (GADVASU), Ludhiana, Punjab, India

Recent estimates suggest that one-third of the annual global burden of rabies ( $\sim 20,000$ cases) occurs in India. With $>95 \%$ of cases due to dog bites, surveillance of animal cases is important to assess the risk to humans and the efficacy of control strategies. We analysed passive surveillance data on 556 samples submitted from 2004 to 2014 to GADVASU in Punjab, India. Most (320;57.6\%) were confirmed rabies cases, including dogs (40.6\%), buffalo (29.7\%) and cattle (23.1\%). Of cases in dogs in which bite history was recorded, $50.7 \%$ had shown biting behaviour. Regression analysis of monthly cases in dogs showed seasonal variation with significant increases in March and August. Although monthly case numbers in buffalo decreased, no long-term temporal trend was detected in dog and cattle cases. Time-series models identified significant cross-correlations between dog and buffalo cases. Significant spatio-temporal clusters were not identified (SaTScan) and mean geographic centres of cases were stable (un-weighted and weighted by species and year). These results demonstrate that overall, the number of rabies cases in animals was temporally and spatially stable during 2004-2014. The endemic nature of rabies transmission in this region demands a coordinated and sustained control program based on a One Health approach.

\section{Can the Sustainable Control of Gastrointestinal Parasites in Small Ruminants Contribute to Improved Child Nutrition in Smallholder Households in Tanzania?}

Mieghan Bruce ${ }^{1}$, Furaha Mramba ${ }^{2}$, Robyn Alders ${ }^{3}$, Julia de Bruyn ${ }^{3}$, Wende Maulaga ${ }^{2}$, Msafiri Kalloka $^{2}$ and Jonathan Rushton ${ }^{1}$

${ }^{1}$ Royal Veterinary College

${ }^{2}$ Tanzania Veterinary Laboratory Agency

${ }^{3}$ University of Sydney

Animal-source foods are some of the best sources of high-quality protein and micronutrients needed for healthy physical and cognitive development, especially among children. Animal health interventions have great potential to improve human nutrition because they can increase income and food security. However, livestock contributions to health and nutrition are complex; the dynamic nature and multiple dimensions of nutrition security require an integrated approach. A system dynamics model was developed to explore the links between gastrointestinal parasites in small ruminants and child nutrition within rural smallholder households in central Tanzania. To quantify the model, household-level data on small 
ruminant ownership and food security is coupled with individual-level data on parasite burden in livestock and nutritional status in children under 24 months of age. There was widespread evidence of gastrointestinal parasites in sheep and goats; $42 \%$ having a heavy parasite burden and associated clinical disease. Despite this, only $7 \%$ of farmers reported controlling parasites in their flock. Model simulations of parasite control strategies predicted reduced livestock morbidity and correspondingly increased household food security and child nutritional status. The results demonstrate proof-of-concept for the utility of system dynamics in the evaluation of the complex linkages between animal health interventions and child nutrition.

\section{7}

\section{Towards Zero Antibiotic Use in Agriculture}

\section{Mark Bryan}

VetSouth Ltd

The New Zealand Veterinary Association has made an aspirational goal that 'by 2030, NZ Inc will no longer use antimicrobials for the maintenance of health and welfare of animals.' This goal was set with the focus of helping mitigate the risk of antimicrobial resistance firmly in mind. New Zealand agriculture already has one of the lowest rates of use of antimicrobials globally, with a recent paper identifying it as the third lowest of those countries with reliable data. However, to achieve the 2030 goal a shift in mindset of the agricultural industry towards a more sustainable approach to production is required.

This paper will report on the current state of the industry in New Zealand with regard to antimicrobial use. In particular, it will discuss monitoring programmes that are being considered, the steps that may be required to achieve the 2030 goal, and the problems that may impede progress. This goal is unique from a global perspective and the journey that New Zealand is starting could provide a valuable template for other agricultural countries.

777

\section{"Lots of Data, Little Information": Beyond Environmental Assessment and Towards the Next Generation of Integrative Cumulative Impact Assessment}

\section{Chris Buse}

$U N B C$

Resource development is a driver of local and international economies and has resulted in extraordinary advances for modern society. As the global thirst for lumber, minerals and energy grows, an increasing amount of stress is placed on the land and its ability to sustain life. With multiple industries now operating adjacent to one another, the cumulative impacts of diverse land-use has become a significant area of scientific inquiry, and it is increasingly recognized that project-based environmental assessment is limited in its ability to capture the impacts of multiple land-uses. This paper reports on the state of the art of cumulative impact assessment in relation to policy developments in British Columbia, Canada. The paper then discusses a new research tool developed by the Cumulative Impacts Research Consortium in collaboration with rural and remote communities with long and storied histories of resource development. The tool is explicitly designed to address shortcomings in the environmental assessment process; to bolster third-party monitoring initiatives at the regional level by integrating environmental, community and health data into a historical understanding of cumulative impacts. Implications for utilizing data across scales and improving an understanding of health and its determinants as related to resource development are discussed. 
475

\section{EcoHealth Scaling up in Latin America: Lessons Learned from Six Vector- Borne Diseases Projects}

Gabriel Carrasquilla ${ }^{1}$, Mauricio Fuentes-Vallejo ${ }^{1}$, Tatiana Garcia-Betancourt ${ }^{1}$, Mariapia Bevilacqua ${ }^{2}$, Vianney de Abrego ${ }^{3}$, Pablo Manrique-Saide ${ }^{4}$, Fabian Mendez ${ }^{5}$, Carlota Monroy ${ }^{6}$ and Concepcion Zuñiga ${ }^{7}$

${ }^{1}$ Fundacion Santa Fe de Bogotá

${ }^{2}$ Asociación Venezolana para la Conservación de Áreas Naturales-ACOANA

${ }^{3}$ Universidad del Salvador

${ }^{4}$ Universidad Autonoma de Yucatán

${ }^{5}$ Universidad del Valle

${ }^{6}$ Universidad de San Carlos

${ }^{7}$ Ministry of Health

The Initiative for Leadership in Ecohealth for Vector Borne Diseases (VBD) in Latin-America supported scaling up experiences for prevention and control of dengue (Colombia and Mexico), Chagas disease (El Salvador, Guatemala, Honduras), and Malaria in Venezuela. We identified factors associated to the process of scaling up. Interviews were conducted to investigators, governmental officers, community leaders and NGOs and private sector. In the analysis we included 1) political context, 2) financing, 3) Stakeholders participation.

In the political context, constraining factors were the instability of the political system, turn over of decision makers and program officers and the difficulty of intersectoral coordination because responsibility is left to the health sector only.

One positive factor was the nature of ecohealth based interventions, because they were addressed not only for the control of the vector but went farther to increase quality of life by improving the dwelling.

Communication about the evidence of results of the intervention and participation of different stakeholders were factors facilitating the scaling up. In addition diffusion of results of intervention strengthens social mobilization that in turn was a way to make pressure to local governments to scale up the interventions to achieve political will and allocation of financial resources.

693

\section{Flaviviruses Detected in Various Species of Mosquitoes Across the Atlantic Forest of Brazil}

Lilian Catenacci ${ }^{1}$, Thito da $\mathrm{Paz}^{2}$, Sharon Deem ${ }^{3}$, Joaquim Nunes Neto ${ }^{4}$, Hamilton Monteiro ${ }^{4}$, Francisco Castro ${ }^{4}$, Elizabeth $\mathrm{Rosa}^{4}$ and Ana Cecilia da Cruz ${ }^{4}$

${ }^{1}$ Post Graduate Program at Evandro Chagas Institute

${ }^{2}$ Universidade Estadual do Pará

${ }^{3}$ Saint Louis Zoo Institute for Conservation Medicine, Saint Louis

${ }^{4}$ Center for Arbovirus Research and Diagnostic Reference at Evandro Chagas Institute

In response to the recent Zika, Chikungunya and dengue virus epidemics, the Brazilian Public Health service has expanded arbovirus surveillance, including in arthropods that may be ecologically competent vectors. The goal of this study was to design and test a screening method for the rapid detection of flaviviruses in mosquito samples. This technique is based on a sensitive reverse transcriptase polymerase chain reaction (RT-PCR) that amplifies a fragment of the flavivirus NS5 gene from infected mosquitoes. Adult mosquitoes in the Southern Atlantic Forest in Bahia, Brazil were captured using CDC light traps, entomological net and an oral suction tube. Mosquitoes were pooled (1-150 individuals) by species, collection site, and date. Laboratory analyses were carried out at the Evandro Chagas Institute, Brazil. From the 233/304 pools that we have 
analyzed to date, 18 generated amplicons and thus confirming the presence of flaviviruses. Various mosquitos species observed in our study carry flavivirus, including: Aedes fulvus, Coquillettidia venezuelensis, Haemagogus janthinomys, Limatus durhamii, Limatus pseudomethysticus, Trichoprosopon digitatum, Anopheles triannulatus, Phoniomyia sp., Sabethes sp., Runchomyia sp., Anopheles sp and Phlebotominae. This study is improving the diagnostic technology necessary for quick arbovirus surveillance in mosquitoes, which in turn will help to inform public health policies in Brazil.

\title{
Social Network Analysis of Poultry Movement and Contact Patterns to Assess Transmission Potential of Exotic Subtypes of Avian Influenza in Poultry- Dense Districts of Bangladesh
}

\author{
Shovon Chakma ${ }^{1,2}$, Kamrul Islam ${ }^{1,2}$, Muhammad Asaduzzaman ${ }^{1,2}$, Md Nurul Islam ${ }^{1,2}$, Mohammad Nizam \\ Uddin Chowdhury $^{1,2}$, Sultan Mahmood ${ }^{1,2}$, Mahbubur Rahman ${ }^{2}$, Ahmad Raihan Sharif ${ }^{2}$, Md Ahasanul Hoque ${ }^{3}$, \\ M Salim uzzaman ${ }^{2}$, Mahmudur Rahman $^{2}$, Sithar Dorjee ${ }^{4}$, Joanna McKenzie ${ }^{4}$, Peter Jolly ${ }^{4}$, Masako Wada ${ }^{4}$ and \\ Roger Morris 5 \\ ${ }^{1}$ One Health Epidemiology Postgraduate Fellow, Massey University, New Zealand \\ ${ }^{2}$ Institute of Epidemiology Disease Control and Research, Directorate General of Health Services, Bangladesh \\ ${ }^{3}$ Chittagong Veterinary and Animal Sciences University, Bangladesh \\ ${ }^{4}$ Institute of Veterinary Animal and Biomedical Sciences, Massey University, New Zealand \\ ${ }^{5}$ MorVet Ltd, Consultancy services in health risk management and food safety policy and programs, Masterton, New Zealand
}

Bangladesh is at risk of introduction of the recently emerged subtypes of avian influenza which are circulating in Asia, having already been affected by the H5N1 subtype. Movement and contact patterns to and from broiler flocks, layer flocks and backyard flocks were investigated in a district of Bangladesh with predominantly commercial flocks, and one with predominantly backyard flocks. Density of poultry in both districts is very high, and they would be severely affected if a novel subtype entered the district. Movement patterns differed in a number of respects between the two districts, and this information has been used to inform the movement parameter settings in a simulation model of avian influenza incursion built in the spatial disease simulation model HandiSpread. The findings provide guidance on control strategies which would be appropriate in the case of an incursion of a novel subtype into each of the types of populations represented by the two study districts.

\section{Knowledge and Practices Towards Rabies: Questionnaire Survey in School Children of Kathmandu District, Nepal}

\section{Rakesh Chand}

National Zoonoses and Food Hygiene Research Centre

A cross-sectional survey was conducted among 351 schools students of grade 8, 9 and 10 of Kathmandu district. With school education being the major source of information (54.2\%), majority (91.5\%) replied that they have heard about rabies, but when specific questions relating to the disease were asked the correct/closest answers kept declining. $80.7 \%$ knew dog bite as transmitting factor and $11.8 \%$ only knew about all the signs of rabies. Dog being the commonest pet (90.4\%), only $75.9 \%$ vaccinated their pets against rabies. $55.4 \%$ pet dogs had access to community/stray dogs. Bite cases were high with $56.7 \%$ children knowing someone who has been bitten and $28.5 \%$ were themselves bitten. 55\% replied of not having provoked the dog and in majority of the instances (69\%) the dog ran away after biting. Hospital based treatments $(72 \%)$ and $\mathrm{PEP} /$ vaccination (64\%) were common. A significant association was seen between knowledge/practice towards rabies 
and type of school, pet ownership and grade of students. These findings demonstrate that although majority of the children knew about Rabies, still there seems to be a knowledge gap. Therefore effective awareness must be conducted starting from the school level as one of the important means of battling rabies in Nepal.

796

\title{
Market and Policy Drivers of Antibiotic Use in Smallholder Periurban Dairy Farms: A Scoping Literature Review Using One Health Approaches
}

\author{
Pranab Chatterjee ${ }^{1}$ and Manish Kakkar ${ }^{2}$ \\ ${ }^{1}$ Public Health Foundation of India \\ ${ }^{2}$ Senior Public Health Specialist, Public Health Foundation of India
}

Antibiotic use in the veterinary sector is a major contributor to the global antimicrobial resistance crisis. From a One Health perspective, policies and market are important components of the environment within which antibiotic usage in veterinary sector takes place. A scoping review was undertaken using the Arksey and O'Malley framework to identify the market and policy drivers of antibiotic use in smallholder periurban dairy farms. The process included: identifying the research question; identifying relevant studies; relevance screening; data charting; collating, summarizing, reporting the data; and expert consultation. Data was iteratively interpreted to develop a conceptual framework. Themes were grouped into three classes: market, farm and systems. Proximal drivers included adoption of intensive production norms, a preference for rearing high-yield exotic breeds in environments with poor hygiene and sanitation, with minimal infection control and biosecurity measures, causing high morbidity levels, necessitating higher antibiotic consumption. Distal drivers included market-level factors like urbanization, population growth, globalized markets, and changing food preferences. Systemic weaknesses, like the absence of antibiotic stewardship, monitoring frameworks, and surveillance and laboratory networks also drove higher antibiotic use. Farm-level antibiotic use is thus driven by a complex interplay of market, farm and systems-level factors needing One Health approach to address them.

\section{8}

\section{Veterinary Antibiotic Use in Smallholder Periurban Dairy Farms of India: A Qualitative Study}

\author{
Abhimanyu Singh Chauhan ${ }^{1}$, Mathew Sunil George ${ }^{2}$, Pranab Chatterjee ${ }^{1}$ and Manish Kakkar \\ ${ }^{1}$ Public Health Foundation of India \\ ${ }^{2}$ Indian Institute of Public Health
}

Although food-producing animals account for a major proportion of antibiotics consumed annually, little is known about the local factors that drive this. This study looked at the knowledge-attitude-practices, influences, and drivers in the key actors in the dairy farming enterprise in peri urban India. In-depth interviews were conducted till attainment of saturation. Coding of core themes was followed by etiological enquiry and generation of a conceptual model. Convergence and divergence of perceptions were mapped across the major stakeholders (dairy farmers, veterinarians, para-veterinarians, traders, pharmacists, and civic officials or union representatives). Cost was a deterrent in seeking care for sick animals; quacks were the major service providers at the farm level. Veterinarians were approached as a final resort. Although civic officials emphasized the availability of laboratory services, farmers denied having ready access. Screening of animals was absent, and routine surveillance was weak. The risk of losing the razor thin profit margins to sickness fueled antibiotic overuse. Direct to farmer marketing, availability of antibiotics over the counter (without or by reusing prescriptions), and informal prescribers providing antibiotics for animal health issues further drove antibiotic. Understanding local dynamics assist in developing sustainable interventions to address the issue of irrational antibiotic consumption. 


\title{
Serological Detection of Bat Coroanvirus Speicific Antibodies in Three Bat Speciese of Taiwan
}

\author{
Yi-Ning Chen ${ }^{1}$, Bo-Gang $\mathrm{Su}^{1}$, Hung Chang $\mathrm{Chen}^{2}$ and Hsi-Chi Cheng ${ }^{3}$ \\ ${ }^{1}$ Chung Yuan Christian University/Department of Bioscience Technology \\ ${ }^{2}$ National Taiwan University/Institute of Ecology and Evolutionary Biology \\ ${ }^{3}$ Endemic Species Research Institute
}

Rhinolophus severe acute respiratory syndrome (SARS)-related CoV, Scotophilus bat CoV 512, and Miniopterus bat CoV 1A were detected in the faeces of 9 bat species in Taiwan by reverse transcriptase polymerase chain reaction (RT-PCR) targeting RNA-dependent RNA polymerase (RdRp) gene. Short infection period of CoV, instable viral RNA, and PCR inhibitor can cause low sensitivity of RT-PCR. To better access CoV epidemiology in the bat populations under surveillance, western blot (WB) and indirect enzyme-linked immunosorbent assay (ELISA) were developed for detecting CoV-specific antibodies. The carboxyl terminal fragment of nucleocapsid protein (N3) without a highly conserved motif among all known CoVs from SARS-CoV and Scotophilus bat CoV 512 were used. Within 52 serum samples from chestnut bats (Scotophilus kuhlii), 26\% was positive by WB and 71\% were positive by ELISA. Within 63 serum samples from Formosan lesser horseshoe bats (Rhinolophus monoceros), $37 \%$ was positive to SARS-CoV, $40 \%$ was positive to Scotophilus bat CoV 512, and $26 \%$ was positive to both CoVs. Only 1 out of 18 Miniopterus bat serum was positive for Scotophilus bat CoV 512 by ELISA. Lactating females had higher detection rate of CoV-specific antibodies. Serological assays were sensitive to assess the infection history of $\mathrm{CoV}$ in bats.

963

\section{Development of Pseudovirus-Based Assay to Investigate the Mechanism for Interspecies Transmission of Bat Coronavirus}

\author{
Yi-Ning Chen and Sheng-Wei Wang \\ Department of Bioscience Technology/Chung Yuan Christian University
}

Bats have been proven to be the natural host of severe acute respiratory syndrome coronavirus (SARS-CoV) so infection studies of bat $\mathrm{CoV}$ on cells of different animal species are required to understand the zoonotic risk of bat CoVs. However, bat CoVs are difficult to isolate and grow in cell culture system. This study aimed to produce bat CoV pseudovirus by using lentivirus-based pseudovirus system. The full length of S gene amplified from Scotophilus bat CoV 512 strain isolated in Taiwan (CYCU-S1/2013) has been cloned as pEGFP-N3-Sco-S. Pseudovirus expressing S protein of Scotophilus bat CoV 512 and green fluorescent protein (GFP) were produce after co-transfection of pEGFP-N3-Sco-S, packaging plasmid and transfer plasmid containing red fluorescent protein (RFP) gene. The production of Scotophilus bat CoV pseudovirus was confirmed by virus infection test on 293T cells, immunofluorescent antibody (IFA) assay against GFP and p24 protein of lentivirus, and electron microscopy. The Scotophilus bat CoV pseudovirus-infected 293T cells expressed red fluorescence and were tested positive for p24 capsid protein of lentivirus. The results showed that lentivirus-based pseudovirus system can produce Scotophilus bat $\mathrm{CoV}$ pseudovirus for further infection studies on cells of different animal species to understand the cross-species transmission of bat $\mathrm{CoV}$. 


\title{
Social, Environment and Climate Change Impacts on Vector-borne Diseases in Arid Areas of Southern Africa
}

\author{
Moses Chimbari ${ }^{1}$, Samson Mukaratirwa ${ }^{1}$, Peter Furu ${ }^{2}$, Barbara Ngwenya ${ }^{3}$, Tawanda Manyangadze ${ }^{1}$, Resign \\ Gunda $^{1}$, Margaret Macherera ${ }^{4}$, Alexio Mbereko ${ }^{1}$, Rose Musesengwa ${ }^{1}$ and Owen Rubaba ${ }^{1}$ \\ ${ }^{1}$ University of KwaZulu-Natal \\ ${ }^{2}$ University of Copenhagen \\ ${ }^{3}$ University of Botswana \\ ${ }^{4}$ National University of Science and Technology
}

We assessed the influence of social, institutional and environmental factors on malaria and bilharzia transmission in vulnerable communities in Botswana, South Africa and Zimbabwe using the ecohealth approach. Quantitative and qualitative data was collected through community and household surveys, epidemiological surveys, GIS and Remote Sensing tools and participatory rural appraisal workshops. Burden of malaria at one study location was very high in 2011 (218 DALYs) but reduced over years to 0.21 in 2015. We demonstrated the value of using the Maxent model to determine snail habitat suitability with small data of presence-only sampling sites. Distance of homestead from piped water collection points, distance from open water sources, religion and toilet use were key determinants of a child being infected with bilharzia. Communities were aware of malaria and bilharzia and how the diseases may be influenced by climate factors. However, knowledge on transmission of the diseases was limited thus compromising community capacity to adequately adapt. Communities claimed that they could predict rainfall based on indicators such as insect and animal behavior and abundance of plants. Engagement between the communities and researchers was very high. Factors influencing transmission of malaria and bilharzia in the context of climate change were analyzed.

\section{Microbial Quality of Dust, Freshwaters, and Ready-to-Eat Fruits and Vegetables Predicts Incidences of Gastroenteritis in Zimbabwe: The Case of Bindura Town}

\author{
Walter Chingwaru ${ }^{1}$, Edwina Mashoko ${ }^{1}$, Tasca Dhori Chingoto ${ }^{1}$ and Jerneja Vidmar ${ }^{2}$ \\ ${ }^{1}$ Bindura University of Science Education \\ ${ }^{2}$ Institute/Zavod Ceres
}

Zimbabwe has been hit by erratic outbreaks of cholera, typhoid and other gastrointestinal diseases in recent years. Capacities of towns and cities in the country to treat wastewaters have become grossly limited due to high urbanisation rates and lack of upgrades to water treatment systems. High average monthly diarrhoea cases were reported in Bindura (30 patients at a clinic; 80 patients at a public hospital; 11 at a private hospital). The following total aerobic counts and E. coli were recorded (respectively): freshwater steams $\left(1.5 \times 10^{5}\right.$ to $\left.1.9 \times 10^{9} \mathrm{CFU} / \mathrm{mL} ;>1.9 \times 10^{4} \mathrm{CFU} / \mathrm{mL}\right)$; cabbage $\left(>3.0 \times 10^{4} \mathrm{CFU} / \mathrm{mL} ; 2.0 \times 10^{3} \mathrm{CFU} /\right.$ $\mathrm{mL})$, tomatoes $\left(5.5 \times 10^{3} \mathrm{CFU} / \mathrm{mL} ; 1.3 \times 10^{2} \mathrm{CFU} / \mathrm{mL}\right)$, carrots $\left(>3.0 \times 10^{4} \mathrm{CFU} / \mathrm{mL} ; 2.3 \times 10^{3} \mathrm{CFU} / \mathrm{mL}\right)$, cucumber $\left(1.6 \times 10^{3} \mathrm{CFU} / \mathrm{mL} ; 2.9 \times 10^{2} \mathrm{CFU} / \mathrm{mL}\right)$, apples $\left(3.9 \times 10^{2} \mathrm{CFU} / \mathrm{mL} ; 0 \mathrm{CFU} / \mathrm{mL}\right)$, dust $\left(2.0 \times 10^{3} \mathrm{CFU} / \mathrm{mL} ; 1.2 \times 10^{2} \mathrm{CFU} /\right.$ $\mathrm{mL}$ at vegetable markets and $<3 \times 10^{0} \mathrm{CFU} / \mathrm{mL} 5 \mathrm{~km}$ away) $(p=0.002)$. All public freshwaters, ready-to-eat fruits (except apples), vegetables, and dust in the town had 'unsatisfactory microbial quality'. Upgrades to the town's wastewater treatment plants and better sanitation are urged for the town to contain diarrhoea. 


\title{
Mainstreaming Biodiversity Conservation and Sustainable Use for Improved Human Nutrition and Wellbeing in Mediterranean Region in Turkey
}

\author{
Ahu Cinar, Saadet Tugrul Ay and Firat Ayas \\ Bati Akdeniz Agricultural Research Institute
}

With over 12.000 plant taxa recorded of which 3905 are endemic, Turkey supports some of the richest plant biodiversity in Europe and Asia. In some parts of Turkey, particularly in rural areas, wild edible plants are collected and used for home consumption or sold in local markets so wild edible plants are not only complementing people's diets but also representing an additional income for many households. Rural people in the Mediterranean Region use many wild plants for nutrition and health. With this study we explore some of these uses. Surveys and questionnaires targeting users, collectors and sellers in rural and urban markets for selected 20 plant taxa were undertaken. Local using procedures and intended uses were documented and plants were sampled for nutrient content analysis. 16 of these plants are commonly used for food while 4 are used for medicinal purposes. In conclusion using wild edible plants for both nutrition and health still plays an important role in rural areas, though knowledge of urban consumers is limited. Nowadays these kind of new choices foods attract attention in markets and this situation causes an ever-increasing livelihood for rural.

1073

\section{Chasing a Shifting Baseline: Reflecting on Surveillance During Disease Emergence Using Lyme Disease in Canada as a Case Study}

Katie Clow ${ }^{1}$, Patrick Leighton ${ }^{2}$, Nicholas Ogden ${ }^{3}$, Robbin Lindsay ${ }^{3}$, David Pearl ${ }^{1}$, Pascal Michel ${ }^{3}$ and Claire Jardine $^{1}$

${ }^{1}$ University of Guelph

${ }^{2}$ University of Montreal

${ }^{3}$ Public Health Agency of Canada

Surveillance for emerging and re-emerging zoonotic and vector-borne disease is critical to assess disease risk and initiate management and prevention measures. However, given the continuous change that accompanies disease emergence, surveillance can be plagued with challenges. In Europe and North America, there has been a notable geographic spread of the vector for Lyme disease, and a subsequent increase in the incidence of disease. We conducted a Realist Review of the literature, using the emergence of Lyme disease in Canada as a case study. Our objectives were to: (1) illustrate the evolution of surveillance approaches across space and time; (2) demonstrate the challenges with past and present surveillance programs; (3) reinforce the value of surveillance; (4) determine the requirements for effective surveillance programs for the future. Based on our review, there appears to be little consistency in surveillance approaches, although many countries have been conducting surveillance for Lyme disease for decades. As a result, limited standardized data are available that can be used to gain a greater understanding into disease emergence and risk. Lyme disease, like other zoonotic and vector-borne diseases, does not respect political boundaries, and we need to move towards a collaborative, integrated and multidisciplinary approach to surveillance.

1071

\section{The Effect of Abiotic and Biotic Factors on the Establishment of Ixodes Scapularis Populations in Ontario, Canada}

Katie Clow ${ }^{1}$, Nicholas Ogden ${ }^{2}$, Robbin Lindsay ${ }^{2}$, David Pearl ${ }^{1}$, Pascal Michel ${ }^{2}$ and Claire Jardine ${ }^{1}$

${ }^{1}$ University of Guelph 
${ }^{2}$ Public Health Agency of Canada

In eastern North America, the hard tick Ixodes scapularis, is the vector for the causative agent of Lyme disease, Borrelia burgdorferi. Within the last two decades the spread of I. scapularis has accelerated northward and this spread is hypothesized to continue, in part due to climate change. Other ecological factors also influence I. scapularis and may play a role in population establishment. The objective of this study was to understand what abiotic and biotic factors are significant in the establishment of I. scapularis in Ontario. Tick dragging was conducted at 154 sites in Ontario during the months of May to October 2014 and 2015. Ecological and georeferenced data was collected for each site. Multi-variable mixed logistic regression models were created to assess the impact of the ecological factors on the presence of I. scapularis. Cumulative annual degree days was positively associated with the presence of I. scapularis, as well as the density of the understory, the presence of shrubs and the interaction of these two ecological factors. Elevation was negatively associated. These findings enhance our understanding of the factors contributing to I. scapularis population establishment, and can be used to enhance current predictive models and risks maps for I. scapularis.

997

\title{
A Model for Integrating One Health into Health Care Delivery Training: The University of Global Health Equity in Rwanda
}

\author{
Jeanne L. Coffin, Janetrix Hellen Amuguni, Peter Drobac, Sarah Trent and Brienna Naughton
}

The University of Global Health Equity (UGHE), operated by Partners In Health, is a groundbreaking health sciences university in Rwanda training the next generation of global leaders in health care delivery. Leveraging expertise from the Government of Rwanda and academic partners including Harvard University, Tufts University and the University of Rwanda, UGHE is an accredited, private institution aiming to utilize innovative pedagogy and multidisciplinary curricula to produce graduates uniquely literate in both global health delivery and equity. UGHE's flagship program, the Master of Science in Global Health Delivery (MGHD), is designed to provide students a robust foundation in global health, One Health management, research methods, and biosocial approaches. The MGHD targets mid-career professionals in medical, nursing, dental, and veterinary science fields, among others, and will also be integrated into the curriculum for future UGHE health professional training programs. By mainstreaming One Health in MGHD's global health delivery training via curriculum, real world case studies, and strategic planning - UGHE ensures students learn to critically examine the complex roles that humans, animals, and the environment play in health outcomes. This model ensures that graduates emerge with a unique ability to address, solve, and plan for the most complex emerging global health challenges.

\section{Agrichemical Exposure in Victorian Farmers - Is There a Human Cost to Maximising Food Production?}

\author{
Jacqueline Cotton ${ }^{1}$, Susan Brumby ${ }^{1}$, Aziz Rahman ${ }^{1}$, Tam Philips ${ }^{2}$ and John Edwards ${ }^{3}$ \\ ${ }^{1}$ Deakin University/National Centre for Farmer Health \\ ${ }^{2}$ Western District Health Service/National Centre for Farmer Health \\ ${ }^{3}$ Flinders University/School of Environment
}

In-field Personalised Cholinesterase Assessment Project (PCAP) is identifying the effect on cholinesterase (ChE) inhibition in Victorian farmers caused by the application of organophosphates (OPs) and ChE inhibiting insecticides. Accurate field assessment is particularly important for remote rural communities and current available measurement does not accurately estimate individual risk. 
Funded by the Shepherd Foundation and commencing in April 2016, this work trials an innovative, transportable assessment tool, identifying farmers with reduced ChE activity, who are at risk of neurological symptoms and/or chronic exposure. Using oxime regeneration to stimulate recovery $\mathrm{ChE}$ in vitro - a more accurate and meaningful estimate of the percentage inhibition of cholinesterase - is generated for the farmer. This new approach will provide farmers with instant results from a measurement tool using their own ChE inhibition range.

Currently being validated across 10 time points in 5 Victorian locations, the study involves 61 farmers and agricultural workers (crop and/or livestock) aged between 20 and 75 years. This presentation will discuss the first 6 months results generated using this novel, objective and portable field test.

Minimising exposure through engagement and education, this research is providing farmers with evidence and fast, individual results, resulting in practice change, attitude shift and prevention of further exposures.

\title{
Bovine TB Control in New Zealand: The Convergence of Human, Animal and Conservation Medicine
}

\author{
Kevin Crews \\ National Disease Manager, OSPRI New Zealand Ltd.; Member of the Australian \& New Zealand College of Veterinary \\ Scientists Epidemiology Chapter
}

New Zealand has had a long history of Mycobacterium bovis (TB) infection in cattle, and latterly deer, herds. This has been largely driven by wild animal infection, primarily possums, an introduced pest into New Zealand which is also responsible for extensive flora and fauna damage. New Zealand has been able to control its bovine TB problem through the targeted control of these infected wild animal populations and at the same time achieve significant collateral benefit to flora and fauna protection. The New Zealand TB control strategy provides a working example of the convergence of human, animal and conservation medicine. This paper will describe the history of the introduction of mammals (wild and domestic) into New Zealand which has led to the establishment of a complex ecological and epidemiological web of bovine TB in man, domestic animals and particularly wildlife species. The same introductions have led to large scale destruction of native flora and fauna. The paper will describe the various plans and strategies implemented over a seventy year period to successfully control bovine TB in New Zealand, including the final phase of the TB eradication programme, recently approved by the Minister for Primary Industries, through to 2055.

946

\section{'Camp dogs': Their Role in the Culture and Health of Australian Indigenous Communities}

Bonny Cumming and Simon Costello

AMRRIC-Animal Management in Rural and Remote Indigenous Communities

Geographic and socioeconomic factors that contribute to the disproportionate life expectancy between Aboriginal and Torres Strait Islanders and non-Indigenous Australians are well documented. Disparity is particularly prominent in remote communities, where access to services is extremely limited. Despite this, Aboriginal and Torres Strait Islander peoples living in remote communities experience an intimate connection to culture and country that many consider essential to their own wellbeing. In remote Aboriginal and Torres Strait Islander communities the social and cultural determinants that underpin the health and wellbeing of both humans and their companion animals must be respected when delivering health services, including veterinary and education programs. This presentation explores dogs as integral to the fabric of Aboriginal and Torres Strait community and culture and the significance of collaborating with communities to develop culturally 
appropriate programs that meet the needs of the community. Additionally AMRRIC - Animal Management in Rural and Remote Indigenous Communities - shares the key attributes for empowering Aboriginal and Torres Strait Islander communities and creating happier, safer and healthier communities.

945

\title{
AMRRIC's Work to Improve Remote Indigenous Community Health Through Veterinary and Education Programs
}

\author{
Bonny Cumming and Simon Costello \\ AMRRIC-Animal Management in Rural and Remote Indigenous Communities
}

AMRRIC - Animal Management in Rural and Remote Indigenous Communities - is a national charity that coordinates veterinary and education programs in Australian Aboriginal and Torres Strait Islander communities. Our work is delivered in some of the most remote regions of Australia, where geographic and socioeconomic barriers preclude permanent veterinary services. In these communities, un-controlled dog and cat populations result in animal health and welfare concerns, biodiversity impacts and impaired human health and safety. By collaborating with remote Aboriginal and Torres Strait Islander communities to improve the health and management of their pets, AMRRIC's work is improving the health and wellbeing of communities, from the individual to the whole. Our work recognises the inextricable links between human, animal and environmental health and aims to deliver integrated veterinary and education programs that cater to the unique socioeconomic and cultural context of remote communities. The immediate objectives of our programs are stable and healthy companion animal populations, however in the long term, our One Health model builds capacity and sustainability so that communities can confidently and effectively manage their own companion animal populations. Through the sharing of skills and knowledge, AMRRIC's OneHealth approach is helping to create happier, safer and healthier Aboriginal and Torres Strait Islander communities.

\section{Oil Palm Production in the Amazon and Socio-Environmental Impacts on Indigenous Lands}

\author{
Sandra Damiani ${ }^{1}$, Carlos Jose Sousa Passos ${ }^{2}$ and Silvia Maria Ferreira Guimarães ${ }^{3}$ \\ ${ }^{1}$ Centre for Sustainable Development, University of Brasilia \\ ${ }^{2}$ Faculty UnB at Planaltina, University of Brasilia \\ ${ }^{3}$ Faculty UnB at Ceilandia, University of Brasilia
}

The expansion of oil palm crops in the Brazilian Amazon rainforest over the last decade seems to be negatively affecting indigenous populations that claim to have been suffering health problems resulting from land-use changes and intensive pesticide use around their lands. The Pará State is the largest Brazilian palm (Elaeis guineensis) producer, and since 2010 it has tripled its production, recently reaching 166 thousand hectares. According to the agro-ecologic zoning for this palm, the plantations can occupy 31.8 million hectares of areas of supposed degraded land in Brazil. Based on official data, it has been estimated that around 332,000 litres of herbicides are annually applied in these crops, which are officially classified as lowimpact activities, with many of them immediately surrounding communities vulnerable to environmental changes. With an anthropological and ecotoxicological approach, we currently seek to comprehend this issue from an indigenous perspective, by analysing how this form of land-use by neighbouring local oil palm companies has been transforming their territories, and possibly affecting their livelihoods and well-being by disturbing the natural resources they rely on (e.g., freshwater 
availability and quality, biodiversity, hunting areas, among others). Our data will help better planning interventions using an ecosystem approach to human health.

\section{4}

\section{Analyzing the Health Value of a Tropical Forest - New Strategies to Mitigate Pandemic Emergence}

Peter Daszak ${ }^{1}$, Tom Hughes ${ }^{1}$, Katherine D. Lee ${ }^{2}$, David Finnoff ${ }^{2}$, Mei Ho Lee ${ }^{1}$, Jimmy Lee ${ }^{1}$, Jon Epstein ${ }^{1}$, Sudarat Damrongwatanapokin ${ }^{3}$, Daniel Schar ${ }^{3}$, Hume E. Field ${ }^{1}$, Carlos Zambrana-Torrelio ${ }^{1}$, Erica Johnson and Allison White

${ }^{1}$ EcoHealth Alliance

${ }^{2}$ University of Wyoming

${ }^{3}$ USAID RDMA

Land use change is a key threat to conservation and public health, through the loss of tropical forest biodiversity and the emergence of known and novel diseases. Efforts to reduce deforestation have focused on estimating the value of ecosystem services, including carbon sequestration, pollination and other services from biodiversity, and incorporating these into land use planning. However, the economic drivers of deforestation and agricultural development often override conservation priorities. Here we describe a new economic modeling approach that explicitly accounts for the cost of disease outbreaks resulting from deforestation. First we show that accounting for the cost of malaria cases associated with land use change in Brazil halves the economic returns from land use change-more than double the impact of loss of ecosystem services. Secondly, we use detailed data on the costs of malaria and other infectious disease cases in Sabah, Malaysia, and ecosystem services valuations to demonstrate that forest conversion represents a net economic loss for the state. Spatial analysis provides potential for land use planning that can provide economic returns on logging and land conversion, reduce public health risk, and optimize conservation benefits. This approach holds the promise of harnessing government, community, and industry commitments to achieve reduced impact land use planning.

1007

\section{How Ecology and Human Behavior Drive Emerging Diseases - Seeking Solutions}

\section{Peter Daszak}

EcoHealth Alliance

The social, economic and environmental drivers of disease emergence are complex and dynamic. They operate against a backdrop of a potentially large diversity of unknown pathogens with an unknown capacity to infect people and become pandemic. All of this is added to a clear lack of capacity or governance to deal with known threats to global health. Given these challenges - what solutions can we envisage to future disease emergence? In this talk I will lay out a growing body of work that allows us to understand, and even predict aspects of disease emergence - from where, from what species, and with what propensity to spread. I will then discuss three types of programs that may help us pre-empt future pandemics at source, or even before they emerge: 1) Pathogen discovery programs; 2) Capacity building programs targeted to the likely origins of future EIDs; and 3) Efforts to identify and reduce the underlying drivers of disease emergence (e.g. land use change, wildlife trade). All of these have significant challenges, significant gaps in our knowledge, and will incur significant cost. However, given the exponentially increasing drivers of disease emergence and the similarly increasing frequency of new EID events, I will demonstrate how these new programs are likely to provide significant return-on-investment. 


\title{
Envisioning Our Future Health: Threats and Solutions \& Introduction to Future Earth
}

\section{Peter Daszak}

EcoHealth Alliance

Local and global environmental change is profoundly affecting our health and environment, and threatens global health progress achieved in recent decades. In many parts of the world, the burden of non-communicable diseases (NCDs) are growing, in addition to the catastrophic impact of disease emergence events as well as unresolved persistent endemic diseases- creating a socalled "double burden" of infectious and non-communicable disease. While the Sustainable Development Goals have a dedicated set of targets to address existing disease threats, preventive systems must be in place to anticipate and more fully address health impacts will result from widescale land conversion and resource extraction, changing food production systems, rapid trade and travel, and climate change. The sustainability research platform Future Earth, launched in 2015, provides a new opportunity for the One Health/EcoHealth/Planetary Health community to engage in research, policy and practice toward a healthier and more sustainable society. Priorities identified in the Future Earth Strategic Research Agenda "Vision 2025” will be examined.

882

\section{One Health in the Anthropocene}

\author{
Katinka de Balogh ${ }^{1}$, Wantanee Kalpravidh ${ }^{1}$, Baldomero Molina-Flores ${ }^{2}$ and Juan Lubroth ${ }^{1}$ \\ ${ }^{1}$ Food and Agriculture Organization of the United Nations \\ ${ }^{2}$ Panamerican Health Organization
}

OneHealth has been widely defined in terms of the need to confront the collective health risks at the animal-human-ecosystems interface. However, in many instances, the environment or ecosystems related discussion is not much more than an afterthought. Today we are living in the Anthropocene era, with human activities having unprecedented impacts on the state of the Earth's biophysical resource base and ecosystems; the prime risk factor in re-emerging 'old' and emerging new diseases. Demographic factors, land use, animal agriculture, and climate change are part of the changing disease landscape and in fact the root causes of these health threats, calling for a cohesive OneHealth approach. The current, interactive session will present various sets of circumstances encountered in a diverse collection of global hotspots. Attendees will be invited to react to the scenarios presented and challenge the panel regarding the options available to tackle the current instabilities and imbalances, in terms of direct disease flare-up as well as the through human action compromised resilience of the concerned planetary systems.

\section{Village Chicken Ownership, Irrespective of Location of Overnight Housing, Has A Positive Impact on Height-for-Age Z-scores of Infants and Young Children in Central Tanzania}

Julia de Bruyn ${ }^{1}$, Peter Thomson ${ }^{2}$, Ian Darnton-Hill ${ }^{3}$, Brigitte Bagnol ${ }^{4}$, Wende Maulaga ${ }^{5}$, Geofrey Kiswaga ${ }^{6}$, Judy Simpson ${ }^{7}, \mathrm{Mu} \mathrm{Li}^{7}$, Siobhan $\mathrm{Mor}^{1}$ and Robyn Alders ${ }^{8}$

${ }^{1}$ Faculty of Veterinary Science \& Charles Perkins Centre, University of Sydney

${ }^{2}$ Faculty of Veterinary Science, University of Sydney

${ }^{3}$ The Boden Institute of Obesity, Nutrition, Exercise \& Eating Disorders, University of Sydney

${ }^{4}$ International Rural Poultry Centre, Kyeema Foundation; Faculty of Veterinary Science \& Charles Perkins Centre, University of Sydney; Department of Anthropology, University of Witwatersrand 


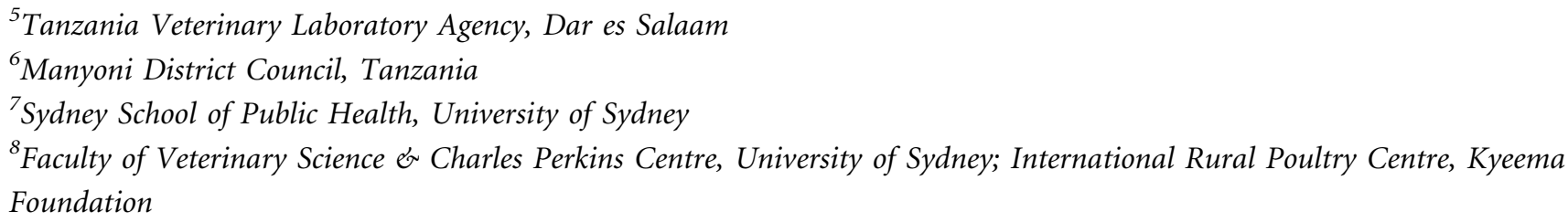

Scavenging poultry transform environmental feed sources into highly nutritious foods for human consumption, of particular value for intrauterine and early postnatal growth and development. Current research is assessing the potential for vaccination programs against Newcastle disease in village chickens to contribute to sustainable improvements in children's nutrition. A total of 510 children $<24$ months of age from eight communities in a semi-arid area of Manyoni District, within the Rift Valley in central Tanzania, were randomly selected to participate in a cluster-randomised controlled trial beginning in May 2014. Sixmonthly data on health and nutrition, household characteristics and livestock ownership, and fortnightly data on chicken numbers and the occurrence of diarrhoea in children, were analysed jointly to assess their impact on height-for-age Z-scores (HAZ). Children from households owning chickens had significantly higher (improved) HAZ than those from households without chickens $(-1.76$ vs. $-1.90 ; \mathrm{p}=0.03)$. Higher HAZ was also associated with improved toilet facilities $(-1.67 \mathrm{vs}-1.99$; $\mathrm{p}=0.02)$ and reduced incidence of diarrhoea $(\mathrm{p}=0.004)$. Separate analysis found no association of diarrhoea in children with chicken ownership $(\mathrm{p}=0.9)$, or with the practice of keeping chickens within human dwellings overnight $(\mathrm{p}=0.2)$. These findings support the potential of poultry-based interventions to improve nutrition in resource-poor settings.

\title{
Health \& Socio-Ecosystems: A Pillar of the Research Platform "Production and Conservation in Partnership" (RP-PCP)
}

\author{
Alexandre Caron, Eddie Mwenje, Mathieu Bourgarel, Daniel Cornelis, Laure Guerrini, Shakkie Kativu, Hervé \\ Fritz, Gift Matope, Billy Mukamuri, Peter Mundy, Prisca Mugabe and Michel de Garine-Wichatitsky \\ Cirad
}

The health of animals and people is a public good, which determines the amount of food, the labour force and well-being of the poorest rural communities. In socio-ecosystems where people coexist with pristine nature, development and conservation objectives need to be concurrently achieved. Health issues at the wildlife/livestock/human interface need to be addressed to mitigate the negative outcomes of these interactions. In parallel, the multi-sectorial dimension of the health issue can be used to trigger more interaction among stakeholders in these socio-ecosystems. Here, we present the Research Platform "Production and Conservation in Partnership", a collaboration between Zimbabwean and French Research institutions launched in 2007, implementing applied research to promote the coexistence between people and nature in Transfrontier Conservation Areas of southern Africa. The platform is currently expending regionally. The "health \& environment" issue is one of the pillars of the platform. We present the community demand-driven, bottom-up applied research agenda that the platform is developing through a local and regional post-graduate training programme. After 10 years of existence, the platform has supported close to 100 postgraduate projects with extensive fieldwork in interface areas and has maintained its objective of scientific excellence through a strong publication policy supported by all partners.

\section{Escherichia coli Population Structure and Antibioresistance at a Buffalo/ Cattle Interface in Southern Africa}

Alexandre Caron, Mathilde Mercat, Olivier Clermont, Méril Massot, Etienne Ruppé, Michel de GarineWichatitsky, Eve Miguel, Hugo Valls-Fox, Daniel Cornelis, Antoine Andremont and Erick Denamur Cirad 
At a human/livestock/wildlife interface, Escherichia coli populations were used to assess the risk of bacteria and antibioresistance dissemination between hosts. We used phenotypic and genotypic characterization techniques to describe the structure and the level of antibioresistance of E. coli commensal populations and the resistant Enterobacteriaceae carriage of sympatric African buffalo (Syncerus caffer) and cattle populations characterized by their contact patterns in the southern part of Hwange ecosystem in Zimbabwe. Our results 1) confirmed our assumption that buffalo and cattle share similar phylogroup profiles, 2) identified a significant gradient of antibioresistance from isolated buffalo to buffalo in contact with cattle and cattle populations; 3 ) evidenced the dissemination of tetracycline, trimethoprim and amoxicillin resistance genes (tet, $d f r A, b l a_{T E M-1}$ in 26 isolated sub-dominant $E$. coli strains between nearby buffalo and cattle populations that led us 4 ) to hypothesize the role of the human/animal interface in the dissemination of genetic material from human to cattle and towards wildlife. The study of antibiotic resistance dissemination in multi-host systems and at anthropised/natural interface is necessary to better understand and mitigate its multiple threats. These results also contribute to attempts aiming at using E. coli as a tool for the identification of pathogen transmission pathway in multi-host systems.

\title{
The Role of Non Government Organizations (NGOs) in the Process of Eradicating Dog Mediated Rabies From Sri Lanka
}

\author{
Ganga de Silva $^{1}$, Nalinika Obeyesekere ${ }^{2}$ and Sivasothy Arumugam ${ }^{3}$ \\ ${ }^{1}$ Director Operations, Blue Paw Trust \\ ${ }^{2}$ Chief Executive Officer, Blue Paw Trust \\ ${ }^{3}$ Additional Director General, Department of Animal Production \& Health, Ministry of Livestock \& Rural Community \\ Development
}

Sri Lanka, a rabies-endemic country, has been trying to control this fatal disease for more than 100 years. Historically the emphasis of control programs have been on Post Exposure Prophylaxis (PEP) though over the past decade new comprehensive intervention measures have been implemented including the successful Colombo City project. This project initiated in 2007 by the NGO, Blue Paw Trust (BPT) together with Colombo Municipal Council and World Society for the Protection of Animals involved mass dog vaccination, sterilization, education and dog managed zones. During five year project period dog rabies cases decreased from 35 to 03. In 2011, the BPT initiated the process together with WHO to develop a National Rabies Strategy with the involvement of Ministry of Health and others. Currently the BPT is assisting the government vets to plan and implement a dog rabies control program for Sri Lanka with a pilot in Eastern Province. The BPT plays the role of mediator and also provides independent monitoring and evaluating to overcome the intrinsic limitations in government managed programs to increase mass dog vaccination coverage from $40 \%$ to the recommended $70 \%$. As a result of inter-sectoral collaboration Sri Lanka has become the SAARC lead country to eradicate rabies.

\section{8}

\section{Nature Relatedness: A Psychological Orientation with Potential to Foster Concurrent Human and Ecosystem Health}

\author{
Julie Dean, Danielle Shanahan, Charles Crockford, Simon Reid and Rich Fuller \\ University of Queensland
}

Increasing urbanisation is leading to reduced contact with biodiverse environments, with fewer opportunities to gain health benefits from contact with nature, as well as emotional disconnection from natural systems. This paper considers the relevance of 'nature relatedness' to ecohealth. Nature relatedness refers to individual differences in the way people view their natural world and their connection with nature, and has been associated with benefits including psychological well-being and pro-environmental behaviour. We present findings from two Australian studies investigating the relationships between 
nature relatedness and health. A study of 1538 participants in a community sample in Brisbane, Queensland considered the relationship between nature relatedness and depression, anxiety, stress and overall health, controlling for a wide range of socio-demographic effects. People with higher nature relatedness had reduced anxiety and better overall health. A study of 251 residents in Cairns, Queensland investigated perceptions of flying foxes including both health risks and ecological benefits of this keystone species. Those with higher nature relatedness were more likely to engage in high risk behaviours such as handling bats, but also indicated greater support for flying fox conservation. We discuss the potential for nature relatedness in public health initiatives to foster both human and ecosystem health.

\title{
221
}

\section{Implementing One Health in Australia: Analyzing Expert perspectives}

\author{
Chris Degeling, Jane Johnson, Michael Ward, Andrew Wilson and Lyn Gilbert
}

To achieve optimal health for humans, animals, and their shared environments, One Health $(\mathrm{OH})$ depends on collaboration between disciplines. Case reports and systematic reviews of $\mathrm{OH}$ success are beginning to emerge. But identifying and assessing barriers to and enablers of effective cross-sectorial collaboration have received scant attention in this literature. We conducted a 4 phase mixed-method Delphi survey with Australian practitioners and policymakers $(n=52)$ to explore areas of conflict and consensus in: (i) how $\mathrm{OH}$ should be defined operationally; (ii) the potential for cross-sectorial collaboration; and (iii) the key priorities that should shape the development of a $\mathrm{OH}$ response to a significant elevation in zoonotic risk. We identified high levels of cross-sectorial consensus on the need for $\mathrm{OH}$ approaches to infectious disease prevention and control, and the key priorities for planning $\mathrm{OH}$ responses to outbreaks. Conflicts occurred in relation to the definition of $\mathrm{OH}$ and the relative importance given to animal health and welfare and economic factors in decision making. Overall the support for a $\mathrm{OH}$ approach across Australian governments and practitioners appears to be strong, but as participants commented, more work needs to be done to ensure crosssectorial differences stimulate better policymaking rather than simply conflict and disagreement.

1035

\section{Three Reasons Why One Health and EcoHealth Need Population Dynamics}

\section{Andrew Dobson \\ Princeton University}

The last ten years have seen a rapid expansion in One Health and EcoHealth; they have achieved this momentum on the thinnest of scientific ice often characterized by weak correlations and over confident specious mapping exercises. If OneHEalth is to truly emerge as a respectable scientific discipline it needs to more warmly embrace a deeper mechanistic understanding of how pathogens are naturally embedded in ecological communities and how disturbance to these systems leads to pathogen emergence. In this talk I'll make some quantitative estimates of levels of pathogen diversity in natural communities; essentially illustrating how trivial it should be to 'discover' new viruses. I'll provide an example of the factors that determine successful emergence and subsequent evolution in a well-studied avian pathogen, before concluding with an analysis of the current and future dynamics of Zika virus and its response to potential control methods.

\section{Challenges and Opportunities for Wildlife Disease Surveillance for Zoonotic Disease Control in Sri Lanka}

Nihal Pushpakumara Don Bamunusinghage ${ }^{1}$, Ashoka Dangolla ${ }^{2}$, Ranjani Hettiarachchi ${ }^{3}$ and Craig Stephen ${ }^{4}$ ${ }^{1}$ Department of Wildlife Conservation, Sri Lanka and Sri Lanka Wildlife Health Center 
${ }^{2}$ Faculty of Veterinary Medicine and Animal Science, University of Peradeniya, Sri Lanka

${ }^{3}$ Department of Animal Production and Health, Sri Lanka

${ }^{4}$ Canadian Wildlife Health Cooperative, Canada

Wildlife play a critical role in emerging and endemic zoonotic diseases. Measures for their prevention, mitigation and early warning require surveillance of wildlife mortality and morbidity, knowledge of the populations at risk and of the changing character of wildlife disease agents. Until recently, Sri Lanka lacked a central wildlife disease surveillance program. The newly created Sri Lanka Wildlife Health Center (SLWHC) brings together ministries responsible for domestic animals, wildlife, human health and the Faculty of Veterinary Medicine and Animal Science of the University of Peradeniya. A questionnaire survey conducted in 2015 and 2016 among field veterinarians, livestock development instructors and wildlife officers $(\mathrm{n}=315)$, indicated that a lack of trained personnel (85\%), inadequate knowledge on wildlife disease (80\%), strict wildlife legislation (60\%), lack of sample collection and storing facilities (95\%), transportation (82\%) and inadequate communication between partner institutes (52\%) were constraints on wildlife disease surveillance. About $70 \%$ of field officers felt they needed additional training. The opportunities for improvement highlighted by the survey included firstly, willingness by $80 \%$ of respondents to participate in wildlife disease surveillance and secondly, the ability through the SLWHC, to improve communication between partners by providing infrastructure, deliver training programs and standardize sample collection, storage and submission.

\section{Quantitative Assessment of Antimicrobial Resistance in Livestock During the Course of a Nationwide Antimicrobial Use Reduction in the Netherlands}

Alejandro Dorado-Garcia ${ }^{1}$, Dik J. Mevius ${ }^{2}$, Jose J.H. Jacobs ${ }^{3}$, Inge M. van Geijlswijk ${ }^{4}$, Johan W. Mouton ${ }^{5}$, Jaap A. Wagenaar ${ }^{6}$ and Dick J.J. Heederik ${ }^{7}$

${ }^{1}$ Institute for Risk Assessment Sciences, Utrecht University//Department of Infectious Diseases \& Immunology, Faculty of Veterinary Medicine, Utrecht University

${ }^{2}$ Central Veterinary Institute, Wageningen UR, Lelystad//Department of Infectious Diseases \& Immunology, Faculty of Veterinary Medicine, Utrecht University

${ }^{3}$ The Netherlands Veterinary Medicines Authority (SDa), Utrecht//Institute for Risk Assessment Sciences, Utrecht University

${ }^{4}$ The Netherlands Veterinary Medicines Authority (SDa), Utrecht, the Netherlands//Pharmacy Department, Faculty of Veterinary Medicine, Utrecht University

${ }^{5}$ The Netherlands Veterinary Medicines Authority (SDa), Utrecht//Department of Medical Microbiology and Infectious Diseases, Erasmus MC, Rotterdam

${ }^{6}$ Department of Infectious Diseases and Immunology, Faculty of Veterinary Medicine, Utrecht University//The Netherlands Veterinary Medicines Authority (SDa), Utrecht

${ }^{7}$ Division of Environmental Epidemiology, Institute for Risk Assessment Sciences, Utrecht University, Utrecht//The Netherlands Veterinary Medicines Authority (SDa), Utrecht

We quantified associations between antimicrobial use (AMU) and acquired resistance in indicator Escherichia coli over a decade (2004 to 2014) during drastic sector-wide antimicrobial use reductions $(\approx 50 \%)$ in main livestock sectors (broilers, pigs, veal calves and dairy cattle) in the Netherlands. We used national data reported annually for the resistance surveillance and AMU program. Two multivariate random-effects logistic models per animal sector were used to relate total and classspecific AMU (as defined daily dosages/animal-year, DDDA/Y) with the probability of E. coli resistance to a panel of 10 antimicrobial agents. Positive dose-response relationships (statistically significant ORs between 1.02 to 1.40) were obtained from all models. In all sectors, total AMU was more associated with resistance phenotypes than class-specific AMU. Resistance to historically widely used antimicrobials (e.g. penicillins, tetracyclines) was, in relative terms, less influenced by AMU changes over time than resistance to newer or less prescribed antimicrobials (e.g. 3rd/4th generation cephalosporins). Thus, drug use history and co-resistance selection were shown as key elements for perpetuation of resistance. The most 
robust associations and positive prevalence predictions related to further AMU reductions were found in pigs and veal calves. The impact of Dutch policies on dairy cows and poultry was more modest.

\section{A Major Reduction in the Use of Antimicrobials for Livestock in the Netherlands Since 2009: The Critical Success Factors}

Jaap A. Wagenaar ${ }^{1}$, Inge M. van Geijlswijk ${ }^{2}$, Johan W. Mouton ${ }^{3}$, Dik J. Mevius ${ }^{4}$, Kees Veldman ${ }^{5}$, Alejandro Dorado-Garcia $^{6}$, Jose J.H. Jacobs ${ }^{7}$ and Dick J.J. Heederik ${ }^{8}$

${ }^{1}$ Department of Infectious Diseases and Immunology, Faculty of Veterinary Medicine, Utrecht University//The Netherlands Veterinary Medicines Authority (SDa), Utrecht

${ }^{2}$ The Netherlands Veterinary Medicines Authority (SDa), Utrecht, the Netherlands//Pharmacy Department, Faculty of Veterinary Medicine, Utrecht University

${ }^{3}$ The Netherlands Veterinary Medicines Authority (SDa), Utrecht//Department of Medical Microbiology and Infectious Diseases, Erasmus MC, Rotterdam

${ }^{4}$ Central Veterinary Institute, Wageningen UR, Lelystad//Department of Infectious Diseases \& Immunology, Faculty of Veterinary Medicine, Utrecht University

${ }^{5}$ Central Veterinary Institute, Wageningen UR, Lelystad, the Netherlands

${ }^{6}$ Institute for Risk Assessment Sciences, Utrecht University//Department of Infectious Diseases \& Immunology, Faculty of Veterinary Medicine, Utrecht University

${ }^{7}$ The Netherlands Veterinary Medicines Authority (SDa), Utrecht//Institute for Risk Assessment Sciences, Utrecht University

${ }^{8}$ Division of Environmental Epidemiology, Institute for Risk Assessment Sciences, Utrecht University, Utrecht//The Netherlands Veterinary Medicines Authority (SDa), Utrecht

In 2008 joint initiatives by the Dutch Government, livestock sectors and the Royal Dutch Veterinary Association (KNMvD) resulted in a covenant describing measures for prudent use of antimicrobials in animals. The Netherlands Veterinary Medicines Authority (SDa) was founded to collect the antimicrobial usage (AMU) data of Dutch livestock, define benchmark targets for AMU in livestock, report annual trends and identify frequent prescribers or misusers. Reduction targets were set by the government at 20,50 and 70\% reduction in 2010, 2013 and 2015, respectively, with reference to 2009. Continuous monitoring of resistance in commensal E. coli had already been set up in livestock from 1998 onwards. The total reduction of AMU (in mass sold) between 2009 and 2015 was $58.4 \%$ and $65 \%$ reduction compared to 2007. The use of antimicrobials defined as "critically important for human health" (fluoroquinolones and $3^{\text {rd }}$ and $4^{\text {th }}$ generation cephalosporins) in livestock has been strongly reduced. As a result of the enforced 1-to-1 relationship of farmers and veterinarians, it was possible to develop the Veterinary Benchmark Indicator allowing to compare prescription levels between veterinarians. Parallel to reduction of AMU there was a reduction of AMR in livestock observed.

\section{Seroprevalence of C. burnetii Among Dairy Cattle Farmers in Chiang Mai, Thailand 2015}

Pawinee Doung-ngern, Punnarai Smithsuwan, Phruetthiphon Sukpom, Somphorn Phornwisetsirikun, Pattarin Opaschaitat, Decha Pangjai, Gilbert Kersh and Pawin Padungtod

Knowledge of Q fever in Thailand is limited. We reported a preliminary analyses of baseline data from a prospective cohort study among dairy cattle farms and farmers in Chiang Mai to describe the magnitude and factors associated with C. burnetii infection among farmers. Two stage random samplings of farms and farmers were performed in five 
dairy cooperatives. Face to face interview with farmers, and blood samples were collected for baseline assessment. Bulk tank milk (BTM) samples were collected from each farm and additional specimens were collected including cows' sera, swab from other animals, and farm environment from the farms with positive BTM. Farmers' sera were tested by Indirect Immunofluorescense Assay. BTM and cow sera were tested by Enzyme-Linked Immunosorbent Assay. Other specimens were tested by polymerase chain reaction. Information of farms and farmers were analyzed using descriptive statistics and Generalized Estimating Equation. Among 637 randomly selected farmers, 532 participated (83.5\%). The overall C. burnetii seroprevalence was 16.9\% (90/532). Working in the farms with BTM positive (OR 2.61, 95\% CI 1.40-4.85), age (OR 1.25, 95\% CI 1.01-1.5), and having contact with cow's birth products (OR 1.95, 95\% CI 0.983.90) were positively associated with the C. burnetii seropositivity. Health education regarding $\mathrm{Q}$ fever prevention to farmers was performed.

\title{
One Health Initiatives for Prevention and Control of Zoonoses in the Kingdom of Bhutan
}

\author{
Kinzang Dukpa ${ }^{1}$, Tenzin Tenzin ${ }^{1}$, Sonam Wangchuk ${ }^{2}$ and Karma Lhazeen ${ }^{3}$ \\ ${ }^{1}$ National Centre for Animal Health, Serbithang Bhutan \\ ${ }^{2}$ Royal centre for Disease Control, Serbithang Thimphu Bhutan \\ ${ }^{3}$ Department of Public Health, Thimphu
}

Bhutan is located in near the Indo-gangetic plains that is notorious for being the hot-spot for emerging diseases such as the Highly Pathogenic Avian Influenza (HPAI) H5N1, Anthrax, and Rabies. Bhutan has experienced clinical cases of HPAI $\mathrm{H} 5 \mathrm{~N} 1$ in poultry; rabies in dogs and cattle; Brucellosis, and Leptospirosis in cattle; and seropositivity against Crimean Congo Haemorrhagic Fever (CCHF) virus in goats thus proving to be a potential indicator for these diseases in the region. Since 2012, Bhutan embarked upon consolidating its One Health Initiatives through a collaborative investigation project funded by the EU and implemented by Massey University. The project strengthened the technical capacity in epidemiological skills; and strengthened local, regional and global collaboration. Disease control documents for HPAI H5N1, Rabies, and Anthrax have been prepared through One Health Approach. The successful control of HPAI H5N1, Rabies, and Anthrax has been possible owing to the success of One Health Initiatives. Bhutan has come up with its One Health Strategic Plan (2016-2020) that consist of key strategies such as institutional set up, disease surveillance, preparedness and response, capacity building, collaborative research, communication, wildlife and environment for implementation of its One Health Programs.

\section{Recent Advances in Defining the Role of Specific Bat Species as Wildlife Reservoirs for Hendra Virus - and Implications When Using Serology to Determine Reservoir Status}

\author{
Peter Durr ${ }^{1}$, Amy Burroughs ${ }^{2}$, Gary Crameri ${ }^{1}$, Kerryne Graham ${ }^{1}$ and Linfa Wang ${ }^{3}$ \\ ${ }^{1}$ CSIRO-AAHL \\ ${ }^{2}$ Department of Health \\ ${ }^{3}$ Duke-NUS Medical School
}

Hendra virus $(\mathrm{HeV})$ was first discovered in southeast Queensland over 20 years ago and has served as an ongoing model for One Health/Ecohealth approaches to bat-borne zoonotic disease investigation and control. Based on serological and virological investigations, all four of the Australian flying fox species have traditionally been considered reservoirs for $\mathrm{HeV}$. 
Recent work - some of it carried out on a colony of grey-headed flying foxes (GHFF) in Geelong - points to this species actually being a "spillover" host and not a primary "reservoir", and this is despite the GHFF being the first bat species in which $\mathrm{HeV}$ was isolated. This finding, if shown to be more generalised, has important implications, as traditionally seropositive status by itself has been used to associate wildlife species as zoonotic reservoirs. Rather our finding indicates that showing sero-positive status is "necessary but not sufficient" when identifying reservoirs, with viral excretion and plausible contact needing to be demonstrated to confirm reservoir status. The need to reassess other zoonotic diseases where multiple bat species have been suggested as reservoir hosts - including MERS-CoV and Ebola - are explored.

670

\title{
Identifying Risk Factors for Nipah Virus Infection in the Southern Philippines
}

Debbie Eagles ${ }^{1}$, Fedelino Malbas ${ }^{2}, E_{d n a}$ Felipe $^{3}$, Catalino Demetria ${ }^{2}$, Edson Simon ${ }^{2}$, Jeneffer Bulawan ${ }^{4}$, Darren Schafer ${ }^{1}$, Stacey Valdeter $^{1}$ and Kim Halpin

${ }^{1}$ CSIRO Australian Animal Health Laboratory

${ }^{2}$ Research Institute of Tropical Medicine, Dept Health

${ }^{3}$ Bureau of Animal Industry

${ }^{4} R A D D L$ XII, Department of Agriculture

In 2014, the first known henipavirus outbreak occurred in the Philippines, in which there were multiple human and horse fatalities. Post-outbreak investigations revealed the most likely routes of transmission for people were from exposure to infected fluids during the slaughter of sick horses, the handling of raw horse meat and very close contact with infected people. A follow-up study has since identified a number of additional possible risk factors for transmission of the virus in this cultural setting. These include the almost ubiquitous consumption of fresh coconut sap, and the high prevalence of consumption of fermented coconut sap (tuba). With collection methods similar to date palm sap, which has been found to contribute to Nipah virus outbreaks in Bangladesh, these require further exploration as possible risk factors. Serological testing of pigs, horses and dogs was also conducted within the study area on the island of Mindanao. All sera tested negative for antibodies to Nipah virus, most likely indicating that the outbreak seen in 2014 was representative of rare spillover of the virus from the reservoir host to domestic animals and people. However, with inevitable encroachment on native ecosystems, these events may become more frequent.

969

\section{Improving Natural Environments and Human Health by Enhancing the Delivery of Environmental Volunteering Programs}

\author{
Matthew Ebden, Rebecca Patrick, Claire Henderson-Wilson and Mardie Townsend \\ Deakin University
}

In Australia environmental volunteers are fundamental to supporting governments, organisations and communities improve and maintain natural environments. They engage in a variety of roles and activities including environmental restoration, environmental monitoring, environmental education, sustainable living, environmental activism and activities that support these areas, such as administration. This paper will report on research that aimed to understand environmental volunteering from the perspectives of volunteers, coordinators and non-volunteers. The research also investigated ways to improve the delivery, coordination and effectiveness of environmental volunteering programs. The research methods comprised of a global review of the literature, in-depth interviews and focus groups involving 46 participants, and surveys of 2744 participants. The research found environmental volunteers are motivated to participate by a variety of factors and that volunteers experience many health and other benefits from volunteering. Most environmental volunteers are satisfied with 
volunteering but much can be done to enhance the experiences of volunteers, volunteer recruitment and retention. Governments and organisations may improve environmental volunteering by developing the capabilities of managers, ensuring a variety of opportunities exist, better using the online environment, improving methods for marketing volunteer opportunities, increasing participation of under-represented groups, building partnerships across government and community, and improving funding and insurance processes.

\title{
919
}

\section{Managing the Health Risks of a Changing Climate}

\section{Kristie Ebi}

School of Public Health, University of Washington

Climate change is one of several global environmental changes that will have far-reaching consequences for animal, plant, and human health across the 21st century. At the same time, development choices will alter underlying vulnerability to these risks, thereby affecting the magnitude and pattern of impacts. The current and projected human health risks of climate change are diverse and wide-ranging, potentially altering the burden of any health outcome sensitive to weather or climate. Climate variability and change can affect morbidity and mortality from extreme weather and climate events, and from changes in air quality arising from changing concentrations of ozone, particulate matter, or aeroallergens. Altering weather patterns and sea level rise also may facilitate changes in the geographic range, seasonality, and incidence of selected infectious diseases in some regions, such as malaria moving into highland areas in parts of sub-Saharan Africa. Changes in water availability and agricultural productivity could affect undernutrition, particularly in parts of Asia and Africa. These risks are not independent, but will interact in complex ways with risks in other sectors. Policies and programs need to explicitly take climate change into account to facilitate sustainable and resilient societies that effectively prepare for, manage, and recover from climate-related hazards.

\section{Zooprophylaxis Contributes to the Interruption of Onchocerca Volvulus Transmission in an Endemic Focus with Intense Cattle Husbandry in North Cameroon}

\author{
Albert Eisenbarth $^{1}$, Alfons Renz ${ }^{2}$ and Daniel Mbunkah Achukwi ${ }^{3}$ \\ ${ }^{1}$ University of Tuebingen \\ ${ }^{2} I R A D$ Wakwa Centre, Ngaoundere
}

Due to onchocerciasis control efforts with mass treatment of the local population with the microfilaricide ivermectin, interruption of the filarial parasite transmission has been confirmed in many American and some African countries. Because in North Cameroon onchocerciasis incidence and transmission has been lower on a highland focus with intense cattle farming compared to an adjacent endemic lowland focus, it has been hypothesized transmission to man is reduced by the presence of animal-borne Onchocerca spp. transmitted by the same vector, a phenomenon called zooprophylaxis. Here we investigated the impact of zooprophylaxis in the very same two foci where vector transmission rates have been surveyed since decades. Microscopic species discrimination of the Onchocerca species was complemented with single-stranded conformation polymorphisms of amplified 16S rRNA sequences to assign unambiguously the species, final host and phylogenetic relationship of nearly all isolated L3's. O. volvulus transmission still prevails in the lowland focus after 29 years of ivermectin treatment, albeit drastically reduced to $3.5 \%$ of baseline. The proportion of animal-borne Onchocerca spp. was $95 \%$. The endemic focus on the highland focus had only bovine Onchocerca spp. in the vector population as soon as 12 years after mass treatment introduction. This clearly demonstrates zooprophylaxis supplements disease control efforts up to the regional interruption of $O$. volvulus transmission. 


\title{
A Place-Based Approach to Farmers' Mental Wellbeing in an Era of Abrupt Climate Change: Notes from the Western Australian Wheatbelt
}

\author{
Neville Ellis \\ Oceania EcoHealth Chapter/Murdoch University
}

Climate change poses a significant mental health risk to Australian family farmers. Previous research examining linkages between climatic adversity (e.g. drought) and farmers' mental wellbeing have tended to highlight economic and social pathways of risk, while overlooking the significance of farmers' place-related attachments and identities for their overall wellbeing. In response, the purpose of this presentation is to highlight place-specific approaches to protecting/enhancing farmers' mental health and wellbeing in the context of abrupt climate change. The presentation draws upon a qualitative community-based case study located in the Western Australian Wheatbelt that sought to understand climate change impacts on family farmers' sense of place and mental wellbeing. The research findings indicate that farmers' sense of place is a significant determinant of farmers' psychological wellbeing, and that interventions that enhance farmers' place-based attachments and identities may deliver cobenefits for community and farmland health. Specific interventions discussed include: 1) negotiating the 'home-work tension', 2) cultural awareness training for new rural health practitioners, and 3) place-sensitive approaches to rural agricultural policy formation. The research findings contribute novel understandings of the determinants of farmers' mental health and highlight the centrality of place in climate change-public (mental) health interventions.

1040

\section{The Ongoing Spreading of AIV-H5 Through Ducks (Anas javanicus) Traded at Sepanjang's Live Bird Market, Sidoarjo, East Java, Indonesia}

\section{A. T. Soelih Estoepangestie \\ Airlangga University}

Ducks and other water fowls have been described as carrier of Avian Influenza Virus (AIV) in the world. AIV-H5 has become endemic in Indonesia since it has been evolved in 2003. Surveillance must be implemented to evaluate and monitor the distribution of AIV and the potential public health problems it may cause in Indonesia, includes ducks meat trading which is one of the most favourites food in East Java Indonesia. To know the role of water fowl as carrier of AIV-H5, the previous study was done to isolate AIV-H5 virus in ducks (Anas javanicus) traded at Sepanjang market, Sidoarjo, East Java, Indonesia. Of totally 156 in three-pooled duck cloacal-swab samples was collected. After isolated in Spesific Anibody Negative (SAN) embryonated chicken eggs Hemagglutination-Inhibition test (HI-test) was done to identify the AIV-H5. The results showed $43(27.6 \%)$ of the samples were positive AIV-H5, and $33(21.2 \%)$ were unidentified. Further identification is needed to identify the untypeable samples.This means that ongoing spreading of AIV-H5and other subtype of AIVin those area were detected which might be lead toreassortment of AIV. Surveillance is needed as an early warning to detectthe emerging of a novel AIV.

\section{Drivers for MERS-CoV Emergence at Human Camel Interface in Qatar}

Elmoubasher Farag, Reina Sikkema ${ }^{1}$, Chantal Reusken ${ }^{2}$, Mohamed Nour $^{3}$, AHmed El-Sayed ${ }^{3}$, Salih Al-Marri ${ }^{3}$, Mohamd Al-Thani ${ }^{3}$, Hazem Ghobashy ${ }^{4}$, Mohd M. AlHajri ${ }^{3}$ and M.P.G. Koopmans ${ }^{5}$

${ }^{1}$ National Institute for Public Health 
${ }^{2}$ Senior Scientist Public Health Virology, Laboratory Preparedness \& Response

${ }^{3}$ Ministry of Health, Doha, Qatar

${ }^{4}$ Ministry of Environment, Doha, Qatar

${ }^{5}$ Erasmus Medical Center and Center for Infectious

The recent discovery of MERS $\mathrm{CoV}$ as a zoonotic disease endemic in dromedary camels raised questions about the possible origin of this infection. Therefore, we set out to review the literature, history and statistics of camel farming, camel Human interface in Qatar, interviews and brainstorming with the stakeholders were conducted, in order to generate hypotheses about the drivers for MERS CoV emergence. The rapid increase in GDP in the past two decades has been paralleled with rapid growth of the production, camel trade and racing industry. As a precious animal, camels are being kissed, hugged, and greeted. Consistent with the disease seasonality, contact with animals intensies during winter time where camel-related activities ourish through race and beauty competitions, trade, camel reproduction and breeding. These activities imply extensive camel movement and mixing along with their owners and workers from all over the Gulf region. Moreover, camel products are used for a variety of domestic purposes. Camel workers live inside camel barns while owners pay regular visits to their barns. Nevertheless, they all strongly deny that MERS-CoV can be transmitted from camels to humans. More in-depth studies were needed to understand the role of social practices in the virus transmission.

\section{Hendra Virus, Horses and Flying-Foxes - Managing the Risk}

Hume Field $^{1}$, David Jordan ${ }^{2}$, Craig Smith $^{3}$, Peter Kirkland ${ }^{2}$, Daniel Edson ${ }^{4}$, Carol de Jong ${ }^{3}$, Deb Melville ${ }^{5}$, Alice Broos $^{6}$, Lee $\mathrm{McMichael}^{7}$, Lauren Goldspink ${ }^{8}$ and Nina Kung ${ }^{8}$

${ }^{1}$ EcoHealth Alliance

${ }^{2}$ New South Wales Department of Primary Industries

${ }^{3}$ Queensland Department of Agriculture and Fisheries

${ }^{4}$ Australian Department of Agriculture and Water Resources

${ }^{5}$ Tolga Bat Rescue and Research

${ }^{6}$ University of Glasgow

${ }^{7}$ The University of Queensland

${ }^{8}$ Queensland Department of Primary Industries

Hendra virus $(\mathrm{HeV})$ causes fatal infection in horses and humans in eastern Australia. Pteropid bats (flying-foxes) are the natural reservoir. We undertook extensive fieldwork to elaborate key aspects of the infection and transmission dynamics in flying-foxes. Viral RNA was detected primarily in two of the four endemic species (Pteropus alecto and P. conspicillatus). Virus was most frequently detected in urine, less so in faeces, and minimally in saliva, nasal discharge, and blood. Spleen and kidney were the tissues most likely to yield virus. Geographically, $\mathrm{HeV}$ prevalence was highest in flying-foxes in southern Queensland/northern New South Wales. Viral RNA was detected in all months, with a marked winter peak in southern Queensland and central and northern NSW, paralleling the spatiotemporal clustering of equine cases. GPS dataloggers showed that flying-fox foraging was repetitious, with a preference for non-native plant species and a resultant increased activity around rural infrastructure. Further, horses used different areas of the paddock night and day, which could contribute to $\mathrm{HeV}$ exposure risk. Satellite telemetry illustrated the mobility of flying foxes and the connectivity of their roosts. Our detailed findings elaborate key aspects of $\mathrm{HeV}$ disease ecology, and allow risk mitigation strategies, including vaccination, to be more effectively targeted. 


\title{
Controlling Q Fever Through a Series of Coordinated Investigations and Interventions Focused on Humans, Goats and the Farming Environment in Victoria, Australia
}

Simon Firestone ${ }^{1}$, Katherine Bond ${ }^{2}$, Michael Muleme ${ }^{1}$, Gemma Vincent $^{3}$, Stephen Graves ${ }^{3}$, Lucinda Franklin ${ }^{4}$, Brett Sutton ${ }^{5}$, Alexander Cameron ${ }^{6}$, Raquel Cowan ${ }^{7}$, Karen Lim ${ }^{8}$, Eugene Athan ${ }^{9}$, Owen Harris ${ }^{10}$, Laura Macfarlane-Berry ${ }^{11}$, Yonatan Segal ${ }^{11}$, Joanne Devlin ${ }^{1}$, Angus Campbell ${ }^{12}$, Tabita Tan ${ }^{12}$, José Canevari ${ }^{1}$, Aminul Islam $^{3}$, Colin Wilks ${ }^{12}$, Jasmin Hufschmid ${ }^{12}$, Glenn Browning ${ }^{1}$, Mark Stevenson ${ }^{1}$ and John Stenos ${ }^{3}$

${ }^{1}$ Asia-Pacific Centre for Animal Health, Faculty of Veterinary and Agricultural Sciences, The University of Melbourne, Victoria, Australia

${ }^{2}$ Communicable Disease Prevention and Control, Department of Health and Human Services, Victoria, Australia; Department of Infectious Diseases, Austin Health, Victoria, Australia

${ }^{3}$ The Australian Rickettsial Reference Laboratory, Geelong, Victoria, Australia

${ }^{4}$ Communicable Diseases Epidemiology and Surveillance, Department of Health and Human Services, Victoria, Australia

${ }^{5}$ Communicable Disease Prevention and Control, Department of Health and Human Services, Victoria, Australia

${ }^{6}$ Meredith Dairy, Meredith, Victoria, Australia

${ }^{7}$ Department of Infectious Diseases, Barwon Health, Australia; St John of God Pathology, Geelong, Australia

${ }^{8}$ Department of Infectious Diseases, Barwon Health, Australia

${ }^{9}$ Department of Infectious Diseases, Barwon Health, Australia; School of Medicine, Deakin University, Australia; Department of Medicine, The University of Melbourne, Australia

${ }^{10}$ St John of God Pathology, Geelong, Australia

${ }^{11}$ Chief Veterinary Officer's Unit, Department of Economic Development, Jobs, Transport and Resources, Victoria, Australia

${ }^{12}$ The Faculty of Veterinary and Agricultural Sciences, The University of Melbourne, Victoria, Australia

Q fever, a zoonotic disease with a complex epidemiology involving farmed and wildlife reservoirs and environmental persistence, threatens human health and an emerging industry in Australia, dairy goat farming. A Q fever outbreak involving a 6000-head goat and sheep dairy enterprise in 2012-2014 required a collaborative interdisciplinary approach into the determinants that rendered this ecosystem vulnerable. A diverse outbreak investigation and management team was constituted including human, animal, environmental and public health agencies, researchers, local government and the affected enterprise. We undertook a series of interviews, risk assessments, site visits and epidemiological studies focused on humans, goats, wildlife and environmental impacts, and implemented coordinated interventions to prevent and control impacts on human and animal health, social well-being and productivity; and to reduce lasting environmental impacts. Several farming and occupational practices were identified as having contributed to the outbreak; some similar factors precipitated the large outbreak in the Netherlands. Local and wide-ranging outcomes of this intervention have included an intensive vaccination program for farm workers, development of a goat vaccine, improved on-farm management, environmental and biosecurity practices, validation of human diagnostics for ruminants and revised regulation of the dairy goat industry. Whilst human cases have ceased; source control interventions are ongoing.

1032

\section{Risk Factors and Determinants of the Geospatial Distribution of Giardiasis in New South Wales, Australia}

\author{
Stephanie Fletcher-Lartey and Soumya Mazumdar \\ South Western Sydney Local Health DIstrict/ NSW Ministry of Health
}

Giardiasis is a common cause of parasitic infection but little is known about the distribution of risk factors, including zoonotic transmission, for giardiasis in Australia. This study seeks to identify the risk factors and the determinants of the 
geospatial distribution of giardiasis in New South Wales. A spatial cluster analyses will be implemented on confirmed giardiasis cases notified to the NSW disease surveillance system from January 2011 to December 2016, in order to delineate areas with high rates of giardiasis. A nested case control study will assess cases notified from January-December 2016, to identify risk factors for giardiasis including zoonotic, water-borne, foodborne and person-person transmission. Preliminary results reveal significant clusters in semi-rural and rural areas as well as urban clusters associated with lifestyle practices. Further analysis will investigate the distribution of giardiasis cases by Local Health District; the association with certain environmental risk factors including proximity to wild-life, use of recycled water, onsite farming, access to municipal water supply, and onsite septic systems and demographic variables. The application of advanced geospatial analysis to the investigation of giardiasis in New South Wales will improve understanding of the epidemiology and geo-spatial distribution of this disease and inform development of appropriate control measures.

1033

\title{
Recurrent Q fever in Occupational Settings: a Case for a Strengthened OneHealth Approach
}

\author{
Stephanie Fletcher-Lartey ${ }^{1}$ and Heidi Lord ${ }^{2}$ \\ ${ }^{1}$ Public Health Association Australia \\ ${ }^{2}$ South Western Sydney Local Health DIstrict/ NSW Ministry of Health
}

Despite the availability of an effective vaccine against $\mathrm{Q}$ fever and legislation to support mandatory reporting of cases in high risk occupational settings, large outbreaks continue to occur in Australia. A cluster of Q fever cases linked to an abattoir was identified in September 2015 by the South Western Sydney Local Health District Public Health Unit. An outbreak investigation employing an OneHealth approach and incorporating active case using a standard case definition, microbiological, environmental and epidemiological assessments was conducted. A confirmed case included detection of Coxiella. burnetti by laboratory methods. Eight cases (seven confirmed and one suspected), all males and currently or having a history of being employed at the same abattoir during their incubation period, reported symptom onset between November 2014 and September 2015. Field investigation identified multiple potential hazards and risk factors at the abattoir and the majority (75\%) of employees were not vaccinated against $\mathrm{Q}$ fever. Widespread lack of risk avoidance and compliance with vaccination requirements were noted. This investigation confirmed the significance of this zoonotic disease as an occupational hazard and identified major gaps in multi-sectoral coordination. An OneHealth approach is needed to strengthen $\mathrm{Q}$ fever control and enforcement of vaccination requirements in high-risk occupational settings.

\section{5}

\section{One Health Aspects of Response to Health Emergencies}

\author{
Heather Fowler ${ }^{1}$, Vickie Ramirez ${ }^{1}$, Janna Schurer ${ }^{2}$ and Peter Rabinowitz ${ }^{1}$ \\ ${ }^{1}$ University of Washington School of Public Health Department of Environmental and Occupational Health Sciences \\ ${ }^{2}$ University of Washington School of Public Health Department of Environmental and Occupational Health Sciences and \\ University of Saskatchewan, Western College of Veterinary Medicine
}

Increasingly, health emergencies are being viewed as One Health opportunities for collaboration and communication between human, animal, and environmental sectors. In practice, however, it can be difficult to overcome operational silos and create a truly coordinated response. The use of emerging communication technologies in these instances may help to resolve some of these barriers. We present an overview of the literature describing recent public health emergencies and the utility of technology in addressing these situations. In addition, we will discuss the cultural and organizational barriers to truly transdisciplinary collaboration that can arise. We will also present an anthropological approach to understanding and overcoming such barriers that incorporates the use of cutting edge communication technology. 


\title{
A One Health Sustainability Metric for Dairy Production
}

\author{
Heather Fowler, Peter Rabinowitz and Vickie Ramirez \\ University of Washington School of Public Health Department of Environmental and Occupational Health Sciences
}

The global production of dairy milk and relate products provides a source of key nutrients needed to sustain an evergrowing global population. At the same time concerns have been raised about the impact of dairy production on the local and global environment. Since dairy farming has critical human, animal, and environmental health aspects, the One Health approach offers a holistic perspective on what can be considered "sustainable" dairy production. Just as the LEED criteria provide an explicit method for rating sustainable building practices, we propose the development of a set of One Health criteria for dairy products that could be eventually used in food labeling to encourage consumers to support sustainable dairy food systems. Such criteria should include aspects such as life cycle analysis to calculate carbon footprint of different production systems and metrics to assess impacts on animal health and welfare, health of dairy workers and consumers and impact on local air, water, and soil environments. We report on initial work toward creating these criteria including development of a conceptual model, qualitative stakeholder assessment, formation of an international advisory board and an evaluation of existing food labels and other dairy production-related metrics.

\section{3}

\section{One Health and Controlling Foodborne Infections}

\author{
Nigel French \\ Massey University
}

In 2015 the World Health Organisation estimated the global burden of foodborne illness to be approximately 600 million illnesses and 420,000 deaths per annum, with a disproportionally high burden experienced in developing countries. Many foodborne pathogens, such as non-typhoidal Salmonella and Campylobacter spp., are maintained in animal reservoirs that transmit infection to humans via food, water and other environmental pathways. Reducing the burden of foodborne infections therefore requires a concerted One Health collaborative effort involving experts from a wide range of disciplines including: medical, veterinary and environmental health; ecology, microbiology; molecular biology; epidemiology; social anthropology; and health economics - working alongside local and national government agencies and the food industry. Understanding the source of foodborne infections is essential for their control and prevention, and this requires identifying the relative contribution of animal reservoirs, transmission pathways and risk factors for infection. Recent advances have led to the development of new tools for 'source attribution', embracing recent developments in pathogen genome sequencing, and evolutionary modelling. This presentation will include examples of the application of source attribution tools in both developing and developed countries, and how they have informed effective policy, leading to a reduction in foodborne disease burden.

\section{1}

\section{Climate Change and Institutional Determinants of Malaria and Schistoso- miasis in Gwanda District, Zimbabwe}

\author{
Peter Furu ${ }^{1}$, Moses Chimbari ${ }^{2}$, Samson Mukaratirwa ${ }^{2}$ and Alexio Mbereko ${ }^{2}$ \\ ${ }^{1}$ Department of Public Health, University of Copenhagen \\ ${ }^{2}$ University of KwaZulu-Natal
}


The spatial and temporal distribution of malaria and schistosomiasis are determined by a series of environmental, biological and social factors including institutional determinants of health. A gap in knowledge exists for some specific institutional determinants and their direct or indirect influence on the epidemiology of malaria and schistosomiasis in Gwanda District, Zimbabwe. Knowledge of these factors is crucial as they may be important confounders in the interpretation of the possible effects of climate change and variability on the two diseases. A detailed inventory and mapping of institutional factors was done with a view to analyse their role in past and present transmission patterns and manifestations in vulnerable population groups. Methods included stakeholder analyses, policy framework analyses, systems analyses and key-informant interviews targeting the sectors of health, environment, agriculture and education. Important sectoral and cross-sectoral policy frameworks, responsibilities and health interventions that may mask the direct effects on disease transmission of climate change and variability and thereby hamper our full understanding of the specific effects of climate change on human health were identified and analysed. Systems, structures and guidelines are in place, however there are operational issues that may influence our understanding of climate change and human health interlinkages.

\title{
617
}

\section{Rabies Transmission Within Wild Dog Populations in Northern Australia}

\author{
Vanessa Gabriele-Rivet ${ }^{1}$, Julie Arsenault ${ }^{2}$, Victoria Brookes ${ }^{1}$ and Michael Ward ${ }^{1}$ \\ ${ }^{1}$ University of Sydney \\ ${ }^{2}$ Université de Montréal
}

Australia is historically free from canine rabies but it is currently spreading through the Indonesian archipelago and is now $300 \mathrm{~km}$ away from northern Australia. Indigenous communities in northern Australia are situated within complex ecosystems and have a high risk of a rabies incursion. They contain large populations of free-roaming domestic dogs and are surrounded by wild dog populations. Therefore, the incursion of rabies into Australia, with spillover to wild dogs, is a genuine threat. The goal of this study is to determine strategies for controlling the spread of rabies in wild dog populations in northern Australia, should an incursion occur. The distribution and density of wild dogs across the study area as well as the extent of interactions between wild and domestic dogs - is being evaluated using a range of field approaches, including motion-activated cameras placed at strategic locations and genetic analysis of fecal samples collected in the bush. A model of rabies spread in wild dogs will be developed and tested using different control scenarios and input parameters. The decision support system developed in this project will help in planning for a potential rabies incursion impacting indigenous communities, wildlife populations and the ecosystem in northern Australia.

22

\section{Women's Health, Livelihood and Biodiversity Conservation}

\author{
Julie Garnier ${ }^{1}$ and Richard Kock ${ }^{2}$ \\ ${ }^{1}$ Odyssey Conservation Trust \\ ${ }^{2}$ Royal Veterinary College
}

Human health, well-being and development are dependent on healthy ecosystems but globally, ecosystems are losing resilience and function as a direct result of land and marine degradation and overexploitation, pollution, expansion of agriculture and human infrastructure. Evidence for this includes climate change, biodiversity loss and the emergence of novel diseases. Efforts to mitigate against these changes include increasing conservation protected areas (PAs). An unintended consequence of this policy is that the costs to adjacent rural communities tend to be local and immediate whilst benefits generated are mostly externalized or inaccessible. Women are particularly vulnerable and are entangled into poverty cycles leading to further environmental degradation and decreased resilience. By examining the roles and responsibilities that local women have in 
managing resources at the human/animal/environment interface, we show how women and children are disproportionately affected by a wide range of health concerns, including infections (zoonotic and anthroponotic) and noncommunicable illnesses. The underlying drivers of disease are limited health care, restricted access to resources, malnutrition, air and water quality, human and animal conflict. We suggest that global conservation strategies need to be reassessed and that the acute problem of poverty with a gender perspective be made a priority around protected areas.

977

\section{Bringing a One Health Approach to Ebola Response and Prevention in Sierra Leone}

\section{Aiah Gbakima \\ Predict}

After more than 28,000 cases and 11,000 deaths, the largest Ebola virus epidemic ever recorded has been nearly brought under control in West Africa. Despite the tremendous progress in controlling the outbreak, the fact that Ebola emerged to affect West Africa in 2013 suggests Ebola virus may have become endemic in the region, potentially by circulation among animal populations. Without the identification of these possible animal "reservoirs and hosts" and development of prevention programs to block transmission from animals to humans, it is likely that future "spillover" of viruses will continue to occur because of continued animal-human contact. The concept of One Health is a collaborative effort to promote and improve the health of humans, animals and our environment through multidisciplinary activities. To avert another Ebola outbreak, a comprehensive, long-term, simultaneous, multi-country coordinated investigation into the potential host range is necessary, including sampling a wide taxonomic range of domestic and wild species. This project, currently underway in Guinea, Liberia and Sierra Leone with assistance from USAID EPT2, may determine how widely distributed the virus may be among animal populations, and how animal host distribution will impact the response and prevention of Ebola in Sierra Leone and other West African countries.

\section{7}

\section{Status of One Health Concept in Veterinary Diagnostic and Investigation Laboratories in Ethiopia}

\section{Solomon Gebre \\ National Animal Health Diagnostic and Investigation Centre}

The National Animal Health Diagnostic and Investigation Center (NAHDIC) of the Ministry of Livestock and Fisheries of Ethiopia is the referral and reference veterinary laboratory in Ethiopia. It is the center of excellence for animal disease surveillance, investigation, diagnosis and research which contributes substantial role in promoting export of animal and animal products, improvement of the livelihood of the smallholder farmers and pastoralists, provision of professional support for investors involved in animal farming and transfer technology for stakeholders. The implementation of the Quality Management System (QMS) in NAHDIC has brought an important milestone for the centre in the face of regional and international community's being as the Regional Referral Diagnostic Laboratory for avian flu and Newcastle diseases. This will have its own impact on international trade of animals and animal products from Ethiopia. Currently, NAHDIC has implemented ISO/IEC17025:2005 in three laboratories selecting 10 tests as its scope of accreditation from SANAS South Africa, since 2009. There are also 14 other regional veterinary laboratories in the country besides many districts, zonal and regional veterinary service clinics providing animal health services for millions of livestock in the country. Emergency response management for zoonotic disease outbreaks of many livestock diseases often involves several different priorities, including protecting animal and human health, food safety and food availability. Disease control measures including 
livestock and poultry quarantines and commodity movement restrictions may disrupt local and international market continuity and food security. Although NAHDIC has started working on surveillance of some zoonotic diseases such as Rift Valley Fever (RVF), Highly Pathogenic Avian Influenza (HPAI), tuberculosis, brucellosis etc. as such it has not been working with concept of one health with other relevant human health and environmental protection Institutions. However thanks to Cambridge university of UK for ETCOBOT project on tuberculosis (bovine and human), DTRA/ ECEB for biosafety and biosecurity and CDC for Brucellosis (human and animal) projects the centre has started working in collaboration with stakeholders dealing human health and environmental protection activities. One health concept has already started in the country some years back by some Universities nevertheless it has not rooted yet in veterinary research and diagnostic laboratories. So the coordinated One Health approach by the human and animal health sectors in Ethiopia will improve the management of zoonoses of public health significance, and ensures the different interests of each sector.

1028

\title{
Plasmid Mediated Colistin Resistance Has Become Wild: Whole Genome Sequencing of Multidrug Resistant E. coli Strain Revealed Putative Zoonotic Connection
}

\author{
Syed Mashkoor Mohsin Gilani ${ }^{1}$, Shahbaz Raza ${ }^{2}$ and Sebastian Guenther ${ }^{3}$ \\ ${ }^{1}$ Institute of Microbiology, University of Agriculture Faisalabad \\ ${ }^{2}$ Institute for Microbiology, University of Agriculture, Faisalabad \\ ${ }^{3}$ Freie Universität Berlin
}

In response to the first report of transmissible colistin resistance mediated by the mor-1 gene in E. coli, we focused on detection of plasmid mediated colistin resistance E. coli from wildlife. Fecal swabs of wintering migratory waterfowls in Pakistan were screened for phenotypic resistance to colistin using VITEK-2 system. RealTime PCR used to confirmed mcr-1 gene. Plasmid conjugation experiment was performed to show colistin gene is transferrable. For genetic characterization, whole genome sequencing (WGS) was carried out on an Illumina MiSeq instrument (Illumina, USA). In-silico analysis WGS data were performed on web service of the Center for Genomic Epidemiology. We found one colistin resistant ESBL-E. coli (MIC $>8 \mathrm{mg} / \mathrm{L}$ ) from migratory Eurasian coot which carried $m c r-1$ gene on IncI2 plasmid. WGS data showed strain carried plasmid resistance genes for beta-lactam (blaTEM-1B, blaCTX-M-15), aminoglycosides (strB, strA, aadA1, aadA2), sulphonamide (sul3, sul2), tetracycline (tetB), trimethoprim (dfrA14). The strains belonged to MLST type ST354. Recently, mor-1-harbouring E. coli of ST354 have also been identified in China from companion dogs and a human with a possible transmission link from dogs to human. The long-range migration of these birds can lead to intercontinental dissemination of $m c r-1$-carrying bacteria.

\section{One Health as Both a Risk and an Approach to Eastern Gorilla (Gorilla ber- ingei) Conservation}

Kirsten Gilardi ${ }^{1}$, Michael Cranfield ${ }^{1}$, Julius Nziza ${ }^{2}$, Eddy Kambale ${ }^{2}$, Benard Ssebide ${ }^{2}$, Linda Lowenstine ${ }^{3}$, Tierra Evans $^{4}$, Joost Philippa ${ }^{1}$, Antoine Mudakikwa ${ }^{5}$, Tracey Goldstein ${ }^{4}$, Christine Kreuder Johnson ${ }^{4}$ and Jonna Mazet ${ }^{1}$ Gorilla Doctors, One Health Institute, UC Davis

${ }^{2}$ Gorilla Doctors, MGVP, Inc.

${ }^{3}$ Gorilla Doctors, UC Davis School of Veterinary Medicine

${ }^{4}$ One Health Institute, UC Davis

${ }^{5}$ Rwanda Development Board, Kigali 
Eastern gorillas (Gorilla beringei) are the most endangered great ape species in the world. At just 880 individuals, the mountain gorilla (G.b.beringei) in Rwanda, Uganda and Democratic Republic of Congo is the only great ape whose numbers in the wild are increasing. This is the result of "extreme conservation" measures centered on park protection, antipoaching patrols and veterinary care. Human habituation of wild mountain gorillas facilitates ecotourism that provides essential revenue to the governments, but brings more than 29,000 people into close contact with mountain gorillas annually. This poses a potential risk for zoonotic pathogen transmission to this endangered species; human pathogens have been confirmed to cause mortality in wild great apes. Habituation also creates an opportunity for One Health interventions: with government partners, Gorilla Doctors provides clinical care to ill and injured gorillas, closely monitors the populations for disease events, cares for confiscated primates, and delivers a preventive health program to park workers. As the implementing partner for the USAID Emerging Pandemic Threats PREDICT project, Gorilla Doctors conducts zoonotic pathogen surveillance and research to better understand diseases in eastern gorillas and inform mitigation strategies for reducing the risk of pathogen transmission between people and gorillas. (word count: 196)

\title{
A One Health Approach for Surveillance of Antimicrobial Resistant Bacteria
}

\author{
Jake Gilliland ${ }^{1}$, Amethyst Holder ${ }^{1}$, Megan Dietz ${ }^{1}$, Mark Sobsey ${ }^{2}$, Samuel Vilchez ${ }^{3}$ and Elizabeth Ryan \\ ${ }^{1}$ ERHS Colorado State University \\ ${ }^{2}$ University of North Carolina \\ ${ }^{3}$ National Autonomous University of Nicaragua, Leon
}

Antimicrobial resistance (AMR) is a major public health concern that merits international surveillance using harmonized approaches across matrices from animals, people and the environment. This interdisciplinary team has identified multiple resistant bacteria using robust and practical methodologies for laboratories across the globe. Wastewater treatment influent and effluent, hospital and community wastewater, recreational waters, livestock, and people were examined for the presence of Methicillin Resistance Staphylococcus aureus (MRSA), Vancomycin-resistant Enteroccocus (VRE), Extended Spectrum $\beta$-Lactamase Enterobacteriaceae (ESBL) and Carbapenem-resistant Enterobacteriaceae (KPC). Relative abundance and AMR properties are measured phenotypically by Kirby-Bauer disk diffusion, isolated with differential and selective agars, and by molecular characterization. Specific results from the enumeration and characterization of AMR strains shared across sample medias and collaborating global sites suggests possible sources and persistence in the environment and community. For example, our preliminary analysis of ESBL showed a $24.06 \%$ positive detection of total coliform in waste water influent compared to $0.03 \%$ positive detection in post treatment waste water effluent. The significance of these findings to veterinary science and human medicine, including agricultural food production, has wide reaching implications for establishing a global One Health surveillance scheme. Funding support provided by Colorado State University One Health Institute.

\section{9}

\section{Social and Cultural Determinants of Health}

\section{Maya Gislason}

Simon Fraser Unviersity

The social and cultural determinants of health are distinct and ideally complementary frameworks which help to articulate the myriad ways in which social and cultural factors impact health and wellbeing and produce differing health outcomes across and between populations, nations and global regions. The social determinants refer to the conditions in which people 'are born, grow, work, live and age' and are particularly focused on the forces and systems which shape these conditions including 'economic policies and systems, development agendas, social norms, social politics and political systems' (WHO 
2016). According to the National Collaborating Centre on Aboriginal Health cultural determinants refer to the systems of meaning that are 'learned, shared, and transmitted from one generation to the next and is reflected in the values, norms practices, systems, ways of life and other social interactions of given culture' (2010, p. 1). Contemplations of health in the nexus between social and cultural factors are necessarily diverse and the voices and wisdom of Indigenous people are integral to this work. In this session a range of theoretical, ontological and epistemological considerations will be given to the subject and a critical and emancipatory ethic will be invited to inform the discussions.

\title{
Cited
}

World Health Organization (2016). 'The Social Determinants of Health'. Accessed on June 24, 2016 at http://www.who. int/social_determinants/en/

National Collaborating Centre for Aboriginal Health (2010). 'Culture and Language as social determinants of First Nations, Inuit and Metis Health'. Accessed on June 24, 2016 at http://www.nccah-ccnsa.ca/docs/fact\%20sheets/social\% 20determinates/NCCAH_fs_culture_language_EN.pdf

\section{The Stop Transboundary Animal Diseases and Zoonoses (STANDZ) Initiative Against Rabies in South-East Asia}

\author{
Mary Joy Gordoncillo and Ronello Abila \\ OIE Sub-Regional Representation for South-East Asia
}

A Grant Agreement between the Government of Australia and the World Organisation for Animal Health (OIE), the STANDZ initiative includes a component addressing dog-mediated rabies in South-East Asia. Under this, STANDZ developed the South-East Asia Rabies Elimination Strategy which was later used as the base document for the joint ASEAN Rabies Elimination Strategy (ARES), and subsequently, the Global Framework for rabies elimination. STANDZ also developed potentially landmark documents relevant to dog rabies elimination including: "Strategic prioritization for mass dog vaccination", "Enhancing animal rabies surveillance through participatory approaches", "Rabies post-vaccination monitoring (PVM)" and "Benchmarking Document for Rabies and Rabies initiatives in ASEAN Member States". STANDZ also supported rabies control initiatives in the Sub-Region with about one million doses of rabies vaccines procured through the OIE Regional Vaccine Bank. STANDZ also supported select countries in implementing mass dog vaccination, public awareness, case investigations, enhanced surveillance, One Health coordination, and political engagement. Other regional and national initiatives include rabies diagnosis capacity building, rabies research, and inter-sectoral coordination. Concluding by the end of December 2016, the STANDZ rabies component will leave South-East Asia with a legacy of materials, capacity, and mechanisms that are all critically relevant in progressing towards rabies-free ASEAN by 2020 .

\section{4}

\section{Relationships Among Dietary Animal Source Food Intake and Disease Status on Measures of Growth in Children Less than 2 Years in Rural Uganda}

\author{
Kellie Curtis ${ }^{1}$, Alexandra Baker ${ }^{1}$, Michael Apamaku ${ }^{2}$, Anita Oberbauer ${ }^{3}$ and Thomas Graham $^{1}$ \\ ${ }^{1}$ Veterinarians Without Borders \\ ${ }^{2}$ National Agricultural Research Organization; Veterinarians Without Borders \\ ${ }^{3}$ Department of Animal Science
}

Chronic malnutrition and illness among pregnant women and children are major modifiable risks to public health and economic development. In Uganda $38 \%$ of children under 5 are stunted and most affected by communicable and other illnesses. These and other factors result in an average IQ in Uganda and SubSaharan Africa 16\% lower relative to developed 
world norms (84 vs 100). We examined 430 Ugandan households in 3 districts to identify effects of animal source food consumption and infection on growth. ASF is consumed less than twice weekly in $50 \%$ of households with $30 \%$ not consuming fish, meat, eggs or milk in their diets. Of 70 males and 61 females under 2 years $(n=131) 30 \%$ were 2 SD under WHO standard for both height and weight for age. However, 54.2\% were 2 SD under standard for height while $32 \%$ were 2 SD under standard for weight. Less than one percent of people had presence of Brucella antibodies. At least $50 \%$ of people older than 21 years were tuberculin test positive. Unexpectedly, 181 individuals were Card Agglutination Test for Trypanosome positive for $T$ gambiense at a dilution of $1 / 2$ or more and 366 were positive on undiluted sera $(n=724)$.

979

\title{
Schistosomiasis and Fascioliasis in Mobile Pastoralists and Their Livestock at Lake Chad: A One Health Approach
}

\author{
Helena Greter ${ }^{1,2}$, Annour Adoum Batil ${ }^{3}$, Bongo Naré Ngandolo ${ }^{3}$, Jürg Utzinger ${ }^{1,2}$ and Jakob Zinsstag ${ }^{1,2}$ \\ ${ }^{1}$ Swiss Tropical and Public Health Institute, Basel \\ ${ }^{2}$ University of Basel, Basel \\ ${ }^{3}$ Institut de Recherche en Elevage pour le Developpement
}

Aiming to sustainably improve human and animal health of mobile pastoralists in the Sahel this project was initiated by the pastoralists' report on economic losses due to fascioliasis in cattle. Fasciola gigantica and Schistosoma bovis are water transmitted trematodes affecting livestock, similar to Schistosoma haematobium and Schistosoma mansoni that cause human disease. By applying One Health research, we investigated these human and animal infections in parallel aiming at elucidating a predictive potential of one to the other and providing the evidence base for One Health intervention strategy development. At Lake Chad, mobile pastoralists of four different ethnic groups participated. In humans $(\mathrm{N}=401)$, Schistosoma haematobium showed a prevalence of $8 \%$. Schistosoma mansoni prevalence was $0.4 \%$. In cattle $(\mathrm{N}=534)$, prevalence of Fasciola gigantica was 31\% and Schistosoma bovis 20\%. Equally in humans and cattle, prevalence correlated within ethnic groups, but varied significantly between ethnic groups. Our results reveal that the mutual predictive value of human schistosomiasis and livestock fascioliasis is defined by transhumance patterns and husbandry system. With a systems understanding of health this shows that taking the ecological and ethnical dimension into consideration is crucial to develop tailor-made locally adapted and accepted interventions, applying the One Health approach.

708

\section{What Drives Rural Poultry Farmers to Implement Avian Influenza Control in Bangladesh?}

Suman Das Gupta ${ }^{1}$, Guillaume Fournié ${ }^{2}$, Md. Ahasanul Hoque ${ }^{3}$, Erling Høg ${ }^{4}$, Tony Barnett ${ }^{4}$, Dirk Pfeiffer ${ }^{2}$ and Joerg Henning ${ }^{1}$

${ }^{1}$ School of Veterinary Science, The University of Queensland (UQ), Gatton 4343, Queensland, Australia

${ }^{2}$ Veterinary Epidemiology \& Public Health Group, Royal Veterinary College (RVC), Hawkshead Lane, North Mymms, Hertfordshire, AL9 7TA, UK

${ }^{3}$ Chittagong Veterinary and Animal Sciences University (CVASU), Khulshi, Chittagong-4202, Bangladesh

${ }^{4}$ Department of Global Health and Development, London School of Hygiene and Tropical Medicine, London, UK

We identify the factors that influence the adoption of effective interventions to control avian influenza (AI) outbreaks among poultry farmers. This involves the use of the Health Belief Model and the Snyder Hope Scale, exploring determinants of AI preventive measures among 108 backyard poultry farmers in Chittagong, Bangladesh. Almost all farmers (> 94\%) acknowledged the severity of AI in poultry, and the contagiousness of AI infection. Farmers were aware of interventions to 
minimise the risk of infection for their flock and themselves, but monetary constraints were reported to impede them from implementing the full range of AI preventive measures. About $72 \%$ of the farmers indicated that the investment cost building separate poultry houses remains a major barrier to AI prevention. We used the Hope Scale, a cognitive model describing the farmer's capacity to initiate actions and to generate routes to reach the goal of controlling AI spread. Statistically significant $(\mathrm{P}<0.05)$ relationships indicated more hopeful groups: 1$)$ farmers who are aware of AI and 2) members of certain religious groups. They may therefore be more likely to explore multiple options for AI prevention. This study suggests that social, structural and individual behavioural determinants should be considered in developing future AI control programs.

\title{
Knowledge, Attitudes and Practices Relating to Brucellosis Among Owners of Small Ruminants in Dohuk, Northern of Iraq: A Venue for One Health Education
}

\author{
Ali Jadaan, Ian Robertson, Anne Barnes and Ihab Habib \\ Murdoch University-School of Veterinary and Life Sciences
}

Brucellosis continues to be a major One Health challenge at the human-animal interface throughout the Middle East, as well as in Iraq. No data on the local knowledge, attitudes and practices (KAP) of Brucellosis in Iraq exist although such information is required for prevention and control measures. We conducted a cross-sectional survey in six districts of the Dohuk Province, in Kurdistan region, Northern Iraq between March and April 2016. We collected information on the socio-demographic characteristics of 72 small ruminants' farmers and captured data on their knowledge, attitude and practice regarding Brucellosis using a structured face-to-face questionnaire.

Only $15 \%$ of the farmers had a good knowledge about Brucellosis. We captured some risky practices, as all of the farmers were found to sell unpasteurised milk directly to consumers, and all did not object feeding the aborted foetus to their dogs. It was remarkable that all of the interviewed farmers showed the same attitude of not separating the animals that had an abortion from the rest of the flock. The knowledge of the small farmers' community in Dohuk, northern Iraq about Brucellosis was very low. Therefore, massive awareness programmes are urgently required to protect the health of people and to limit the economic burden of Brucellosis on their animals.

1082

\section{Poor Access to Care Among Snakebite Impoverished Victims and Their Animals}

\section{Abdulrazaq Habib \\ Arinex Pty Ltd}

Snakebite envenoming (SBE) is a major cause of morbidity and mortality among rural dwellers and agricultural workers throughout the tropics and the developing world. Farmers and livestock herders are at highest risks of poisoning and its burden is underestimated. In West African savannah nomadic Fulani and their cattle herds, and domestic animals are at an increased risk of SBE. It is also common among plantation workers, traditional and pastoral societies in forested and other ecosystems. Envenoming presents mainly with pains, swelling, bleeding, anemia, paralysis and organ failure. Without antivenom treatment, death may result in $>20 \%$ of victims and disabilities such as amputation, blindness and psychological disturbances are common. Antivenom developed from animal sera is lifesaving but is generally neither accessible, available nor affordable to victims especially in remote areas. Delay to access effective antivenom predicts death and marketers exploit impoverished vulnerable populations with inappropriate products further worsening outcome. The burden of human SBE is similar and in some regions much higher than for other encountered Neglected Tropical Diseases. 
Although antivenom is also effective on envenomed animals and livestock, the problem is much underappreciated. Access to antivenom, care and prevention should be prioritized for humans and animals where SBE is common.

\title{
328
}

\section{Demand for Online Education in One Health}

\section{David Hall and Mary Zhou}

This study examined the demand from health professionals for online One Health education topics, preferred formats for electronic learning, and available communication and knowledge exchange technologies. A combination of 20 semi-structured interviews and an online questionnaire completed by 500 participants across Canada were used for data gathering. The topics highest in demand were quantitative epidemiology and statistical methods. Although definitions of One Health were provided, limited understanding of the concept of One Health (as opposed to integrated health disciplinary studies) may have biased those results. The majority of respondents (65\%) had at least an undergraduate degree in a health science field. More than $50 \%$ of respondents were planning to take a Continuing Education course in the next 12 months and of those, more than $85 \%$ were willing to pay more than $\$ 150$ for the online course in One Health. More than $65 \%$ of respondents expected some form of certification from the course. The study provided some insight to learner stated preferences for online One Health education course format, engagement with online learners, and monitoring and evaluation.

\section{6}

\section{Water Public Health on Small Scale Farms in Vietnam}

\author{
David Hall \\ Quynh Ba Le
}

We examined the relation between water public health, small scale integrated farming, and mitigation of emerging infectious diseases (EIDs) using a transdisciplinary participatory approach in Vietnam. Data were collected using participatory methods from 600 farms in North and South Vietnam (Thai Binh and An Giang provinces) using questionnaires, semi-structured interviews, and water quality testing procedures (E. coli, turbidity, and $\mathrm{pH}$ ). Water samples were collected from participants' wells or rain water cisterns and analyzed in government laboratories using WHO standardized methods. Probit analysis was used to investigate the association of demographic variables with levels of E. coli in drinking water and EID mitigation strategies. Farmers raised fish, poultry, a few pigs or cattle, and some crops. Most participants had basic awareness of avian influenza prevention, but limited knowledge of water-borne diseases. More than $80 \%$ of samples contained unacceptable levels of E. coli (10 to several thousand cfu's). Variables significantly associated with unacceptable levels of E. coli included age $(\mathrm{p}<0.01)$, presence of and number of livestock on farm $(\mathrm{p}<0.01)$, history of vaccinating poultry against $\mathrm{H} 5 \mathrm{~N} 1(\mathrm{p}<0.05)$, and declared interest in public health training $(\mathrm{p}<0.01)$. Increased transdisciplinary approaches to increasing awareness of water public health and livestock waste management is recommended.

\section{Bayesian Belief Networks and Water Public Health in Vietnam}

\section{David Hall \\ Quynh Ba Le}

Bayesian Belief Networks (BBNs) model cause and effect relationships between deterministic and probabilistic variables. We developed a BBN using data gathered from 600 small scale integrated farmers in Vietnam to examine relationships between 
predictive variables and levels of E. coli in drinking water from wells and rain water. Sensitivity analysis of the model revealed that choice variables were particularly likely to influence endpoint values, reflecting the highly variable and impactful nature of preferences, attitudes, and beliefs relating to mitigation strategies. This BBN model of SSI farming in Vietnam was helpful to the understanding the complexity of small scale Vietnamese agriculture as well as for identifying and estimating impact of policy options. The BBN model also provided insight to the influence of subjective choice variables and deterministic factors including preferences for learning, understanding of public health concepts, livestock, income, and years of farming on the likelihood of contaminated drinking water. Attention to rural water public health management policies in Vietnam has been limited in scope. This BBN and other policy tools can assist in understanding the role policy can play in helping SSI farmers understand options for engaging in water public health mitigation strategies that do not disrupt their chosen livelihoods.

957

\title{
Zoonotic Diseases in China in an Era of Climate Change
}

\author{
Alana Hansen ${ }^{1}$, Peng Bi ${ }^{1}$, Qiyong Liu $^{2}$ and Jianjun Xiang ${ }^{1}$
}

${ }^{1}$ The University of Adelaide

${ }^{2}$ State Key Laboratory of Infectious Disease Prevention and Control, Collaborative Innovation Center for Diagnosis and Treatment of Infectious Diseases, National Institute for Communicable Disease Control and Prevention, Chinese Center for

Disease Control and Prevention

Zoonotic diseases transmitted by arthropods and rodents can be a serious public health issue in China. We sought experts' opinions on China's capacity to meet the environmental and social challenges of emerging and re-emerging zoonotic diseases in a changing climate. Face-to-face interviews were conducted with 30 infectious diseases experts in four cities in China. Two vector-borne diseases (dengue fever and malaria) and a rodent-borne disease (haemorrhagic fever with renal syndrome) were discussed. A thematic analysis identified strengths and shortcomings of the current system. Participants expressed optimism in China's future capacity to manage these diseases. However, health literacy in the population is lacking, research capacity could be strengthened, and improved training and conditions for staff involved in surveillance and response would aid capacity building. Early warnings of disease outbreaks would be useful, and prompt and coordinated responses are required when outbreaks occur. It was also considered important that health professionals remain skilled in the identification of diseases such as malaria for which incidence is declining in China, so that re-emerging or emerging disease trends can be rapidly identified and interventions activated. The study's recommendations may be useful to policymakers in China and the Asia Pacific region.

925

\section{Is a National One Health Disease Surveillance System Possible?}

\section{Peng Bi, Alana Hansen, Philip Weinstein and Scott Cameron}

The University of Adelaide

The risk of emerging and re-emerging disease outbreaks in Australia and the Asia Pacific region will be amplified in coming decades due to drivers such as demographic changes, increased travel, globalisation of food production, intensive animal husbandry, increased antibiotic resistance and a changing climate. With the likelihood that serious epidemics of emerging zoonotic diseases could arise in coming years, a One Health approach is increasingly being considered the most appropriate way to manage these threats. Australian communicable disease notification systems have to date focused primarily on human diseases. This presentation will discuss the benefits and barriers to integrated veterinary and public health surveillance linking animal and human data. Whilst this has been successful in managing zoonoses to some extent at state 
level, a national system is yet to be realised. Issues such as information sharing, security issues, the challenges of data linkage; and the potential need for a national coordination authority will be discussed.

\title{
Empowering People in Surveillance Systems: Strategies to Motivate and Enable Farmers, Communities and Veterinary Service Personnel
}

\author{
Syibli $^{4}$ \\ ${ }^{1}$ Australian Government Department of Agriculture and Water Resources \\ ${ }^{2}$ AusVet Animal Health Services \\ ${ }^{3}$ Australia Indonesia Partnership for Emerging Infectious Diseases \\ ${ }^{4}$ Indonesian Ministry of Agriculture
}

Jonathan Happold ${ }^{1}$, Angus Cameron ${ }^{2}$, Catriona Mackenzie ${ }^{2}$, Ben Madin ${ }^{2}$, Albertus Muljono ${ }^{3}$ and Muhammad

Biosecurity risks are increasing as a consequence of agricultural intensification, global trade, climate change and other factors, and the need for surveillance for livestock diseases and zoonoses is growing. Within domains of expertise, much is known about surveillance methodologies, information and communication technologies, and sociocultural factors that influence disease reporting. However, few examples exist where cross-disciplinary collaboration has progressed beyond a pilot stage and delivered transformative change in surveillance capacity at a national level. This presentation explores the reasons why investments in surveillance systems that do not address technical, technological and social dimensions are unlikely to be effective or sustainable. Reasons include the tendency to focus on the technical and technological domains and ignore the central role that people play in surveillance systems, as contributors and users of surveillance information. Conversely, the implementation of strategies that motivate and enable people at every level of the surveillance system - from the farmer to the central policy-maker - can substantially enhance surveillance capability and health service delivery. We discuss recent experience in Indonesia which demonstrates how a multidisciplinary team put these theories into practice to establish a national animal health information system that currently receives more than 7,000 reports per day.

\section{Converting Lawns to Gardens: Improving Health and Food Security for New Refugees in the U.S.}

\author{
Kari Hartwig and Meghan Mason \\ St. Catherine University
}

Refugees and new immigrants in a country often struggle with learning a new language, laws and systems while trying to find jobs, housing and also coping with past traumas. Community gardens offer physical and mental health benefits to alleviate some of these stressors for immigrants. In this project, refugee settlement agencies partnered with urban and suburban churches to convert their lawns to gardens and create new community garden space to immigrants in the Twin Cities of Minnesota, USA. This study reports on the evaluation of this project using pre and post surveys and focus groups with gardeners. The surveys had a $44 \%$ response rate. There was a statistically significant increase in vegetable intake throughout the day between the pre and post surveys. Only 3\% of gardeners indicated food insecurity issues but $84 \%$ participated in one or more food subsidy program. Qualitatively, gardeners indicated the value of the gardens to improve their mental and physical health, and the social connections. The results illustrate the value of gardening for improved physical and mental health of refugees and strengthened social connections. In addition, the model of converting lawns into gardens has implications for creating a more sustainable use of the environment. 


\title{
Exploring Mental Health Services Among Climate Victims in a Cyclone Affected Area of Coastal Bangladesh
}

\author{
M Tasdik Hasan ${ }^{1}$, Gourab Adhikary², Sultan Mahmood ${ }^{3}$, Nowshin Papri ${ }^{2}$, Abul Kalam Azad ${ }^{4}$ and Mahbuba \\ Nasreen $^{4}$ \\ ${ }^{1}$ International Centre For Diarrhoeal Disease Research, Bangladesh/Public Health Foundation of Bangladesh (PHFBD) \\ ${ }^{2}$ International Centre for Diarrhoeal Disease Research, Bangladesh \\ ${ }^{3}$ One Health Epidemiology Fellow, Massey University, NZ-IEDCR, Dhaka, Bangladesh \\ ${ }^{4}$ Institute of Disaster Management \& Vulnerability Studies, University of Dhaka (IDMVS), Bangladesh
}

In 2014 German Watch declared Bangladesh as the 5th ranked country in climate risk. Every year it is attacked by cyclone creates huge impact on mental well being but this issue is still a neglected topic. We conducted this exploratory study with a view to explore the situation of mental health services in a cyclone affected village. A social mapping, KII with different stakeholders (10) \& IDI with affected people (10) were done for identification of location of health care service centers, activities of government institution, NGOs, local volunteers, informal health care providers, resources \& possible future steps related to mental health care. It was revealed that natural disasters like cyclone had many psychological impacts on population. People have suffered by post traumatic stress disorders, anxiety, panic, acute stress reaction, sad feelings, suicidal thoughts \& children and female were more vulnerable. The government/NGOs had no specific plan of action/ initiative to address these issues. There was a visible gap in thinking about finding of an effective way to give the people proper psycho social services. To make resilient \& responsive health systems for this vulnerable group of population, implementation of effective mental health program with a strong mental health policy is needed.

1006

\section{Is Exposure to Animal Feces Harmful to Child Nutrition and Health Outcomes? The Evidence so Far}

\section{Derek Headey \\ IFPRI}

This paper reviews existing evidence on whether exposure to livestock is a significant risk factor for child nutrition and health outcomes in poor countries. Formative research from several countries suggests that poultry may be a particular risk, because poultry stay close to the household and defecate in areas where children habitate. Two studies found that children often directly ingest chicken feces, and a third found that $28 \%$ of mothers reported that their children had eaten soil in the past 2 weeks. A three-country nutrition study reported that animal feces were visible in $38-42 \%$ of household compounds, and that this indicator was negatively associated with height-for-age $\mathrm{Z}$ scores in models that adjusted for confounding factors. Surveys from Ethiopia and Bangladesh recorded whether or not poultry were kept in the main household dwelling overnight. Both found negative associations between these indicators and child growth, and the Bangladesh study also found a positive association with markers for environmental enteric disorder. Recent data from 40 countries shows that poultry are the most commonly owned livestock in poor countries. Poultry ownership is therefore likely to be an important risk for child health outcomes, though more research is needed on these issues. 


\title{
One Health, One Lab, One Prototype for Success
}

\author{
Warren Hendrickson \\ $H D R$
}

HDR has developed a prototype laboratory 'kit of parts' design approach consisting of a series of laboratory and supporting space 'Modules' capable of supporting the majority of scientific activities typically present in public and animal health testing laboratories. HDR has used this approach on projects globally including Kenya and Iraq. The modules were designed with consistent dimensions to easily fit together, akin to LEGO blocks. The modules incorporate current international best practices and are designed to ensure the potential for a sustainable approach utilizing local trades, methods and materials. Three dimensional drawings and plans, annotated with equipment requirements, are used for each module. The intent is that the modules can be used in early stage design meetings to allow users to make well informed selections of spaces to quickly realize a schematic design with sufficient information to develop accurate space, service and budget requirements. The primary advantage of this unique approach is to shorten the time and reduce overhead costs related to the design phase. The goal has been to develop a system that allows flexibility to address unique risks in unique situations, while taking advantage of the quality and consistency inherent in a well-developed, repeatable, and sustainable design.

\section{4}

\section{Taenia Solium from a Community Perspective: Baseline Costing Data in Katete/Sinda Districts in Eastern Zambia}

Emma Hobbs ${ }^{1}$, Kabemba Evans Mwape ${ }^{2}$, Brecht Devleesschauwer ${ }^{3}$, Carol Bulaya ${ }^{2}$, Chembensofu Mwelwa ${ }^{2}$, Isaac Phiri ${ }^{2}$, Maxwell Masuku ${ }^{2}$, Gideon Zulu ${ }^{4}$, Angie Colston ${ }^{5}$, Arve Lee Willingham III ${ }^{6}$, Amos Chota ${ }^{2}$, Dirk Berkvens, Pierre Dorny, Emmanuel Bottieau and Nicolas Speybroeck

${ }^{1}$ Ross University School of Veterinary Medicine/Institute of Tropical Medicine Antwerp/Ghent University

${ }^{2}$ University of Zambia

${ }^{3}$ WIV-ISP Belgium

${ }^{4}$ Ministry of Health, Government of the Republic of Zambia

${ }^{5}$ GALVmed

${ }^{6}$ Ross University School of Veterinary Medicine

The zoonotic tapeworm Taenia solium is highly endemic in Zambia, however the societal cost of the parasite is unknown. Baseline economic costs related to T. solium cysticercosis in people and pigs were measured in fifty percent of randomly selected households in the study villages. One questionnaire $(n=245)$ captured individual costs related to clinical signs commonly attributable to human T. solium infections. A pig questionnaire $(\mathrm{n}=271)$ captured herd demographic data, costs of pig-keeping, and economic losses from porcine cysticercosis. Human health data revealed 30 (12.2\%) cases of seizure-like episodes, 90 (36.7\%) of severe chronic headaches, and $54(22.0 \%)$ of blurry vision or blindness, leading to 137 health care consultations and 16 hospitalisations in a five-year period. Hospitalisation costs ranged from $\$ 0$ - $\$ 570$ USD per visit. 489 working days were lost due to the surveyed conditions per year. Median monthly household income is \$0USD (mean \$9.20USD, range \$0-\$250USD). Twenty-five percent of surveyed households keep pigs, mostly free-ranging. Median pig-keeping costs are $\$ 0.50$ USD per pig per month. Over $95 \%$ of adult pigs are sold to local traders, who commonly perform tongue palpation before purchase. 94.5\% (256/271) of survey respondents cannot sell tongue-positive pigs; infected pigs that are sold lose $45 \%$ of their value. 


\title{
One Health Ecohealth Science Approaches Underpinning Human Health and Security in New Zealand: One Organisation's Journey
}

Virginia Hope, Graeme Benny, Libby Harrison, Rob Lake and Phil Carter

ESR-The Institute of Environmental Science and Research

The Institute of Environmental Science and Research in New Zealand (ESR) provides human health, environmental and forensic science support for government agencies in New Zealand. Across the three areas, the human-animal-environment interface is an increasingly relevant aspect of our operational and research work. ESR also shares the location for its outbreak and pandemic related services with animal health experts working for the Ministry for Primary Industries at the National Centre for Biosecurity and Infectious Disease which has led to a range of joint operational activities and research projects. The development of genomic approaches to the management of communicable disease in humans and animals and in water and food quality monitoring has further enhanced our ability to work across this interface in the interests of human health. Investigation and attribution of the burden of zoonotic diseases by ESR is relevant to a number of government agencies, and benefits from taking an integrated health perspective. This presentation will track the growth in importance of One Health Ecohealth aligned activities both across ESR and with external collaborators, and describe support for translation into policy and action.

\section{1}

\section{Areas of Potential Zoonotic Disease Emergence in Africa Related to Live- stock, Wildlife Diversity and Human Density, With Projections to 2050 Based on Shared Socioeconomic Pathways}

\author{
Chia Yi Hou ${ }^{1}$, Carlos Zambrana-Torrelio ${ }^{2}$, Peter Daszak ${ }^{2}$, John Mumford ${ }^{3}$ and L. Roman Carrasco ${ }^{4}$ \\ ${ }^{1}$ National University of Singapore; Imperial College London \\ ${ }^{2}$ EcoHealth Alliance \\ ${ }^{3}$ Imperial College London \\ ${ }^{4}$ National University of Singapore
}

The interface between livestock and humans may play a large role in zoonotic disease emergence. This work aims to understand how differences in Africa's potential future pathways of development may affect infectious disease emergence through intensification of livestock production. Zero-inflated Poisson (ZIP) models of emerging infectious disease (EID) events were developed based on past events, socioeconomic and environmental variables that span human, livestock, and wildlife populations. To represent demand for protein, Shared-Socioeconomic Pathways (SSPs) projections were produced for volume of meat and eggs using projections of human population and GDP to 2050. ZIP results highlight the importance of pig density, wild mammal diversity, and forest cover change to explain zoonotic disease emergence. Sheep and goats are also important livestock groups. Emergence hotspot countries were identified, such as Cameroon and Uganda. The largest increases in projected meat and eggs occurred in SSP5 (high challenges to mitigation and adaptation to climate change) followed by SSP1 (low challenges to mitigation and adaptation). An SSP case study analysis showed areas in Chad, Ghana, and Kenya with high probability for EIDs relating to projected increased pig and human population density. This work may provide direction for scenario development and zoonotic EID preparedness. 


\section{The Necessity of Mobile Phone Technologies for One Health Surveillance in Benin}

Yaovi Mahuton Gildas Hounmanou ${ }^{1}$, Murielle S. S. Agonsanou ${ }^{1}$, Mahugnon H. B. Vodougnon ${ }^{1}$, Ephraim M. Achoh $^{1}$, Tamegnon Victorien Dougnon ${ }^{1}$ and Ezron D. Karimuribo ${ }^{2}$

${ }^{1}$ University of Abomey-Calavi

${ }^{2}$ Sokoine University of Agriculture

A cross-sectional study was conducted in March 2016 to assess the need of mobile phone technologies for health surveillance in Benin. 25 medics, 33 vets and 72 respondents from the public participated from Southern Benin. Questionnaires were used to assess possession, use and need of mobile phone in health surveillance and interventions in Benin. All respondents possess cell phones and respectively 75, 84 and 100\% of the public, medics and Vets already use them for medical purposes. $75 \%$ all respondents acknowledged that the traditional surveillance systems in the country are ineffective and do not capture and share real time information. More than $92 \%$ of the respondents confirmed that mobile phones have potential to improve health interventions and surveillance in the country. Furthermore, all respondents adhered to a nascent project of mobile phone-based health surveillance and confirmed that there is no existing similar approach in the country. However, the most preferred methods by respondents for effective implementation of such platform are phone calls (96.92\%) followed by SMS (49.23\%) and smart phone digital forms (41.53\%). This calls for the development of mobile phone-based surveillance network in Benin for timely and efficient one health surveillance leading to tropical diseases elimination.

\section{An Invitation to Speak: Using Social Science Methods to Dig Deeper into Complex Invasive Species Issues}

\section{Tanya Howard \\ Australian Centre for Agriculture and Law, University of New England}

Invasive species in Australia have economic, environmental and agricultural impacts. They take hold in landscapes that are segmented by land tenure boundaries, fragmented governance regimes and short-term planning cycles. Current management and control approaches are informed by technical expertise in species ecology, however successful implementation also requires sustained and coordinated collective community action. This paper emerges from a multi-disciplinary research program that integrates behavioural science, institutional analysis and community engagement scholarship to build more effective and equitable strategies for invasive species governance. The program seeks to augment technical and scientific knowledge with social science that explores the human dimensions of invasive species management. Specific pest vertebrate species such as feral pigs and wild dogs are highly mobile in the landscape, creating disputes over where responsibility for control should lie. Using narrative interview and analysis techniques, case studies uncover stories of success, complexity and trade offs, particularly in relation to biodiversity and agricultural productivity. Analysis shows that land managers hold diverse and nuanced perspectives about invasive animal control, the role of collective action and the barriers and opportunities for improvement in management practices. This paper presents empirical data and makes suggestions for application of these findings in front-line community engagement practice. 


\title{
"SOS: Summer of Smoke. A Mixed-Methods, Community-Based Study Investigating the Health Effects of a Prolonged, Severe Wildfire Season on a Subarctic Population"
}

Courtney Howard ${ }^{1}$, Caren Rose, $\mathrm{PhD}^{2}$, Warren Dodd ${ }^{3}$, Katherine Kohle, MD ${ }^{4}$ and James Orbinski, OC, MSC, Bsc, MD, $\mathrm{MA}^{5}$

${ }^{1}$ Canadian Association of Physicians for the Environment

${ }^{2}$ University of British Columbia

${ }^{3}$ University of Guelph

${ }^{4}$ Yellowknife Health and Social Services

${ }^{5}$ Balsillie School of International Affairs

Between June 15-Aug 31 2014, wildfires burned 3.4 million hectares of forest in Canada's Northwest Territories (NWT), yielding Canada's most severe urban smoke exposure for Yellowknife, the subarctic capital, and surrounding aboriginal communities. We analyzed PM2.5 levels, salbutamol dispensations, clinic and hospital cardiorespiratory variables, and analyzed in-depth video interviews with community members. $49 \%$ of days June15-Aug 31 in 2014 had a PM2.5 over 30 $\mathrm{mcg} / \mathrm{m}^{3}$, as compared to 3\% in 2012 and $9 \%$ in 2013 and 2015 . Max daily PM 2.5 in 2014 was $320.4 \mathrm{mcg} / \mathrm{m}^{3}$. There was a $22 \%$ increase in outpatient salbutamol dispensations in 2014 compared to the average of 2012, 2013 and 2015. More cough, pneumonia and asthma were seen in clinics compared to 2012-2015 ( $\mathrm{P}<0.001)$. There was a $42 \%$ increase in respiratory ER visits in 2014 compared to 2012-2013, but no change in cardiac variables. The respiratory effect was most pronounced in children 0-4 (114\% increase in ER visits). Qualitative information shows a reduction in traditional activities, and that severely-affected people left. Respiratory and cardiac findings are consistent with previous studies. Further mixed-methods studies are required to detail whole-picture health effects of wildfires in order to best target public health interventions. Funding: Health Canada-Aboriginal Health Branch.

\section{Bat-Human Interactions in Human Populations Highly Exposed to Bats in China: Implications for Zoonotic Disease Transmission}

\author{
Huimin Huang ${ }^{1}$, Shiyue $\mathrm{Li}^{1}$, Zhengli $\mathrm{Shi}^{2}$, Hong $\mathrm{Yan}^{1}$ and Xinglou Yang ${ }^{2}$ \\ ${ }^{1}$ School of Public Health, Wuhan University \\ ${ }^{2}$ Wuhan Institute of Virology, Chinese Academy of Sciences, Wuhan, China
}

Nowadays bats have been proposed as major reservoirs for diverse emerging zoonotic diseases. To explore the relationship between human-bat interactions and virus infection, a survey with standard questionnaire was conducted in July 2014 and January 2015 in Xianning, Hubei province in China. Blood and oropharyngeal swabs were also collected. Of 1098 respondents with an average age of 56 (range: 4-93), 564 (51.4\%) lived in Taiyi Dong near the bats cave, 647 (58.9\%) were women, and $84.2 \%$ were farmers. Animal exposures in the past year included seeing bat around house [13.5\%];having bat fly into house [27.8\%]; going to bat cave for fun [13.8\%]; hunting or getting bite by bats [2.8\%]. In the past half year, influenza-like illness were described by $380(34.6 \%)$ participants. Individuals who lived near the bats cave have a higher rate of contacting with bats (including seeing bat around house, having bat fly into house, going to bat cave for fun) than those who are not $\left(\chi^{2}\right.$ were 19.6,10.5,89.8, p value were $<0.001,0.001,<0.001$ respectively). For virus detection, no batCoronavirus infection was found. Sharing of living spaces may lead to higher chance of human - bat interactions. But exposure to zoonotic pathogens has not yet found. Long term of surveillance of pathogens circulating in both highlyanimal-exposed human and animal population is still needed. 


\title{
Wildlife as Sentinels for Ecosystem Health - Why Skippy's Lumpy Bones May Matter
}

\author{
Jasmin Hufschmid $^{1}$, Clare Death ${ }^{1}$, Graeme Coulson ${ }^{2}$ and Ian Beveridge ${ }^{1}$ \\ ${ }^{1}$ Faculty of Veterinary and Agricultural Sciences, The University of Melbourne \\ ${ }^{2}$ School of BioSciences, The University of Melbourne
}

In the context of One Health, we most commonly think of infectious diseases; however, a range of non-infectious diseases including toxicities may reflect on ecosystem health, an essential component of the One Health concept. Wildlife toxicology is a relatively recent field, but toxicities in free-ranging wildlife often indicate broader issues within the ecosystem, including environmental pollution. Chronic exposure to elevated levels of fluoride have been demonstrated to cause significant pathology and disease in affected human populations. We investigated bone and dental pathology and clinical signs of lameness in a range of marsupial species resident near two fluoride-emitting industrial facilities in southern Victoria and found these to be consistent with chronic fluoride toxicity indicating elevated levels of fluoride in the environment immediately adjacent to the facilities. The monitoring of wildlife for evidence of toxicity may thus be used to flag pollution issues potentially impacting on human populations, especially where fluoride emitting industry is located in close proximity to residential areas.

1020

\section{Diversifying Food and Diets: Using Biodiversity to Improve Global Nutrition}

\section{Danny Hunter \\ Bioversity International}

One of our greatest challenges is ensuring everyone has access to a healthy and affordable diet that is produced in a sustainable manner. Despite advances our global food system still fails to feed a significant part of humanity adequately. While transformations in global agrifood value chains have made a greater variety of food commodities available to consumers in many countries around the globe, they have also led to greater homogeneity in the global food system, compromising dietary diversity and environmental sustainability. The enhanced use of biodiversity is a cornerstone of an integrated approach to address the 'triple burden of malnutrition'. Countries such as Brazil have shown by strategic actions and interventions that it is possible to create better environments to mainstream biodiversity for healthier diets and improved nutrition into government programs and public policies. The 2030 Agenda for Sustainable Development presents an opportunity to reshape food systems and move beyond business-as-usual towards sustainability and the provision of healthy diets, however, at present the importance of biodiversity for healthy agriculture, food systems and diets is not adequately reflected in the SDGs.

964

\section{Lyssavirus: Does Australia Bite into More Than its Fair Share of the World's Immunoglobulin?}

\author{
Penny Hutchinson ${ }^{1}$, Margaret Young ${ }^{2}$, Kari Jarvinen ${ }^{3}$ and James Smith \\ ${ }^{1}$ Darling Downs Hospital and Health Service \\ ${ }^{2}$ Wide Bay Hospital and Health Service \\ ${ }^{3}$ Metro South Hospital and Health Service
}


${ }^{4}$ Metro North Hospital and Health Service

Since discovery of Australian bat lyssavirus (ABLV) in 1996 and human deaths now totalling three, Australia has aggressively managed potential lyssavirus exposures. This has led to significantly greater use of Human Rabies Immunoglobulin (HRIG) in post exposure treatment as defined by current guidelines, and usage has not been curtailed despite a welldocumented chronic global shortage of HRIG and scarcity of the product in countries with endemic canine rabies and high rates of human deaths. Disproportionate HRIG use by risk-averse wealthy countries with low rates of human lyssavirus infection is an issue of global distributive justice. There has been no appetite in Australia to re-assess the tolerance of risk to potential lyssavirus exposures. With Australia's worsening obesity epidemic and increased travel to rabies-endemic areas, the volume of HRIG use will only increase. This presentation argues that current guidelines for management of potential lyssavirus exposures both in Australia and other industrialised nations raise ethical dilemmas that must be addressed. This presentation argues for a unified approach to rigorous risk stratification of potential exposures, according to factors such as mode of exposure (bite vs. scratch), species of animal (non-human primates and rodents), geographic location of exposure and site of injury.

\section{6}

\section{Assessment of Exposure to Toxic Metals in Children From Aquiles Lanza Ghetto-Montevideo, Uruguay}

Fiorella Iaquinta ${ }^{1}$, Nelly Mañay ${ }^{1}$, Adriana Cousillas ${ }^{1}$, Cristina Alvarez ${ }^{1}$ and Mariela Piston ${ }^{2}$

${ }^{1}$ Toxicology Department, Universidad de la República, Facultad de Química

${ }^{2}$ Analytical Department, Universidad de la República, Facultad de Química

Recently a childreńs health survey showed blood lead levels of concern in Aquiles Lanzás gheto. The families were not well advised about this environmental problem and they were worried about their children. These families showed their concern to an NGO, Gurises Unidos that takes care of this ghetto working with the families living there, by taking actions to improve their children's quality of life. Owing to this children's lead health risks issues, families' demanded urgent solutions, so the Toxicology Area of Chemistry Faculty, proposed a joint project with the NGO, based on EcoHealth pillars. This project́s aims were to assess lead and other toxic metals exposure, such as manganese, arsenic and mercury in childreńs hair samples and their possible correlations with health, social and environmental status. Physicians, social workers, teachers, biologists, chemical toxicologists and a biochemist integrated the research team. This team prepared didactic materials to present in several workshops with this community in order to inform and prevent toxic metals exposure. In the meantime, hair samples and individual interviews were taken and laboratory analysis were carry out afterwards. We present the analytical results and statistical processing data with our conclusions and learnings to continue working on Ecohealth approach within these environmental health problems in Uruguay.

671

\section{Modelling the Problems of Rabies Elimination in the Philippines Using One Health}

Van Joe Ibay ${ }^{1}$, Ro McFarlane ${ }^{2}$ and Colin D. Butler ${ }^{3}$

${ }^{1}$ Animal Welfare Coalition Manila, Provincial City and Municipal Veterinarian League of the Philippines, Public Health

Association of Australia

${ }^{2}$ University of Canberra, Faculty of Health

${ }^{3}$ University of Canberra, Health Research Institute and Australian National University, National Centre for Epidemiology and Population Health 
The Philippines has adopted a national, collaborative strategy, involving health experts and government agencies, seeking to eradicate rabies by 2020. Through the "Anti-Rabies Act of 2007", the National Rabies Prevention and Control Program was established. A central strategy for this was One Health. In support, an Administrative Order established the Philippine Interagency Committee on Zoonosis, enabling cross-sectoral and cross-disciplinary collaboration in various levels of government and society. The National Rabies Program has divided the Philippines into 18 regions with 81 provinces in order to pursue stepwise rabies elimination. Of these provinces, 35 have since been declared rabies free zones. Between 2007 and 2015, the number of suspected rabies cases declined by approximately 35\%, from 3,379 submitted samples to 2,207. This presentation examines the system collaboration and mechanism which bridged the human and animal health professions to work with the various stakeholders and agencies at each levels of government. By exploring the various experiences of key stakeholders, we aim to investigate the nature of the collaboration, and to examine the potential and sustainability of One Health as a strategy to address other zoonotic disease issues in the Philippines such as ebola Reston, avian influenza and leptospirosis.

\title{
On the Diversity of Arbovirus Circulating in Monkeys Habituating in Selected Kenyan Urban Centres
}

\author{
Maamun Jeneby ${ }^{1}$, Jandouwe Villinger ${ }^{2}$, Daniel Masiga ${ }^{2}$ and Rosemary Sang ${ }^{3}$ \\ ${ }^{1}$ Institute of Primate Research \\ ${ }^{2}$ International Centre of Insect Physiology and Ecology-icipe \\ ${ }^{3}$ Arbovirology/VHF Unit, CVR/KEMRI, Nairobi
}

Urban centres in East Africa have witnessed increasing mosquito-borne virus (arbovirus) outbreaks in recent decades. The objective of this study was to investigated, for the first time, the diversity of zoonotic arbovirus circulating in free-ranging Chlorocebus aethiops (African green monkey - AGM) and zoophilic mosquitoes within selected urban centres in Kenya, East Africa. A total of $103 \mathrm{AGM}$ and 1500 pools of mosquitoes were screened for arboviruses by virus isolation in cell culture, RT- PCR, nested PCR-high resolution melting analysis and sequencing. Molecular exploration data demonstrate that Chikungunya, Sindbis, Usutu, Bunyamwera and Semliki forest viruses circulate in free-ranging NHPs and zoophillic mosquitoes (Aedes aegypti, Culex pippiens and Culex zombaensis) within Kisumu and Mombasa urban centre in Kenya, East Africa. Retrospectively, the surveillance data from this study yields useful information on possible source of arbovirus infection in humans residing in populated African urban areas.

\section{4}

\section{Health at the Sub-catchment Scale: Typhoid and Its Environmental Determinants in Central Division, Fiji}

\section{Aaron Jenkins \\ Edith Cowan University}

The impact of environmental change on transmission patterns of waterborne enteric diseases is a major public health concern. This study concerns the burden and spatial nature of enteric fever, attributable to Salmonella Typhi in Central Division, Republic of Fiji at a sub-catchment scale over 30-months (2013-2015). Quantitative spatial analysis revealed relationships between environmental conditions of sub-catchments and incidence and recurrence of typhoid. Average incidence per sub-catchment for Central Division was high at 205.9/100 000, with cases recurring in each calendar year in $26 \%$ of sub-catchments. Numbers of cases were highest within dense, urban coastal sub-catchments, but incidence was highest in low-density mountainous rural areas. Significant environmental determinants at this scale suggest increased risk 
of exposure where sediment yields increase following runoff. Small populations living near small, erosional, high-energy headwaters and small streams unconnected to large hydrological networks, appear at reduced risk compared to large systems that broaden into meandering mid-reaches and floodplains with alluvial deposition. This study indicates that anthropogenic alteration of land cover and hydrology (particularly via fragmentation of riparian forest and connectivity between road and river networks) affects the transmission of typhoid and that environmental (long-cycle) transmission of typhoid is important in Fiji.

\title{
Targeting Surveillance for Zoonotic Viruses Based on Risk of Disease Spillover and Spread
}

Christine Johnson ${ }^{1}$, William Karesh ${ }^{2}$, Peta Hitchens ${ }^{3}$, Tracey Goldstein ${ }^{4}$, Damien Joly ${ }^{5}$, Andrew Clements ${ }^{6}$, Peter Daszak ${ }^{2}$ and Jonna Mazet ${ }^{4}$

${ }^{1}$ EpiCenter for Disease Dynamics, One Health Institute, School of Veterinary Medicine, University of California - Davis

${ }^{2}$ EcoHealth Alliance

${ }^{3}$ Swedish University of Agricultural Sciences

${ }^{4}$ One Health Institute, School of Veterinary Medicine, University of California - Davis

${ }^{5}$ Metabiota

${ }^{6}$ Global Health Security and Development Unit, Bureau for Global Health, U.S. Agency for International Development

Emerging zoonotic diseases continue to present a significant burden to global health, particularly where dense human populations and pressures on environmental and economic resources are greatest. To establish a risk-based surveillance strategy to detect emerging diseases that threaten human health for the Emerging Pandemic Threats PREDICT project, we examined past trends in animal hosts and transmission mechanisms involved in spillover of zoonotic viruses and amplification by human-to-human spread. We find that viruses with high host plasticity (i.e. taxonomically diverse host range) were more likely to amplify viral spillover by secondary human-to-human transmission and have broader geographic spread. Viruses transmitted to humans during circumstances that facilitated mixing of diverse animal species had significantly higher host plasticity. We also identified a strong linear relationship between species abundance and viral spillover, with the more common species, especially those increasing in abundance, having transmitted more viruses to humans. Our findings suggest that surveillance should be focused at high-risk interfaces that facilitate mixing of animal species in areas with high biodiversity, dense human populations, and land use change that facilitates frequent contact between people and wildlife, particularly highly adaptable species increasing in abundance.

\section{Implementing a One Health Approach to Emerging Infectious Diseases: Understanding Public Priorities and Values Using a Discrete Choice Experiment}

\author{
Jane Johnson, Gwendolyn Gilbert, Kirsten Howard, Michael Ward, Andrew Wilson and Chris Degeling \\ University of Sydney
}

There is increasing consensus within the scientific community that a One Health approach is the most effective way of responding to threats from emerging and re-emerging infectious diseases (EIDs). However, statutory reviews of past EID events have consistently shown that successful implementation of policies for managing EID outbreaks and risks actually depends on how well these policies align with public values. That is, despite its empirical merit, a One Health approach will 
not succeed if it does not take into account how it will be understood, interpreted and judged by the public; a One Health approach needs to reflect what most Australians will accept as fair, and good for public health. This paper will report on a Discrete Choice Experiment (DCE) involving 2000 Australian citizens, designed to test what members of the public are willing to trade-off to prevent and control outbreaks of EIDs. These results have the potential to inform and improve the implementation of One Health approaches; this paper is the first report of our findings.

495

\title{
Produce and Protect? Extractive Industry Tensions in a Sustainable Development Agenda
}

\author{
Lesley Johnston ${ }^{1}$, Jennifer Jones ${ }^{2}$, Colleen Davison ${ }^{3}$, Oyunaa Lkhagvasuren ${ }^{4}$ and Craig Janes ${ }^{1}$ \\ ${ }^{1}$ University of Waterloo \\ ${ }^{2}$ University of Guelph \\ ${ }^{3}$ Queens University \\ ${ }^{4}$ Simon Fraser University
}

The Sustainable Development Goals direct efforts to ensure sustainable consumption and production patterns, and to protect, restore and promote sustainable use of terrestrial ecosystems. These two goals reveal vexing tensions and represent a compelling challenge for effective and public-health informed governance of extractive industries. Juxtaposing the imperatives of environmental preservation and sustainable development, which includes protecting associated social, cultural and health resources, offers an opportunity to explore how these spaces are being created in historically underserved and underserviced regions. Tools such as environmental, social and health impact assessments, impact and benefit and agreements and regulatory processes that require free, prior and informed consent, with their many opportunities and challenges, begin to offer more systematic, and hopefully just, approaches to the charting of these spaces, while raising many questions, including about the role of equity, participation, adequacy of information, consent and conflict. These questions will be examined and examples will be drawn from work conducted in health impact assessment practices from the Southern Gobi region of Mongolia, and with the impact benefit agreement processes in Northern Canada and Alaska, USA, to explore whether and how sustainable consumption and protection of ecosystems might coexist.

\section{3}

\section{Isolating Geohelminthiasis Infection in School-Aged Children Who Work in Artisanal Mining in North Kivu, Drc}

\author{
Jean paul Kabemba lukusa ${ }^{1}$ and Lesley Johnston ${ }^{2}$ \\ ${ }^{1}$ Volunteers for the Consrvation of Fauna and Flora \\ ${ }^{2}$ University of Waterloo
}

Health is closely related to the quality of the environment in which people live. Geohelminthiasis infections are among the most common infections in the world, affecting the poorest communities. The WHO recommends periodic deworming for children (1-15 years) living in areas where the prevalence of infections is estimated at more than 20\%. In Democratic Republic of Congo, especially in the underserved region of North Kivu, many children working in artisanal mining may not receive necessary treatment. The goal of this study was to identify the parasite carriage in this group. We collected and analyzed 125 fecal samples from children aged 6-15 who work in artisanal mining. We found that $65.6 \%$ of children have Ancylostoma duodenale, 94.4\% have Trichuris trichiura, and 96.8\% have Ascaris lumbricoides, and many of them have 
never been dewormed. Our research demonstrates the immediate need for a deworming program and health education for disease prevention in the North Kivu mining region. The ultimate goal of our work is to eliminate child mining, given the risk of exposure to multiple diseases, injury, and radiation exposure common in artisanal mining, though the challenge is great given the prevalence of poverty and war in region

917

\title{
One Health Rotation for Inter-professional Health Sciences Students
}

\author{
Malika Kachani $^{1}$, Maryam Othman ${ }^{2}$, Japhet Magambo ${ }^{3}$ and Eberhard Zeyhle ${ }^{3}$ \\ ${ }^{1}$ Western University of Health Sciences, College of Veterinary Medicine, California \\ ${ }^{2}$ Western University of Health Sciences, College of Osteopathic Medicine of the Pacific, California \\ ${ }^{3}$ Meru University of Sciences and Technology, Meru
}

Stemming from the concept that "One Health creates a framework for educating the next generation of health professionals", the College of Osteopathic Medicine (COMP) and the College of Veterinary Medicine (CVM) at Western University of Health Sciences (WUHS), California, have initiated a One Health Rotation for fourth year students. The goal of this rotation is to present the students with opportunities and skills that prepare them for the changing landscape of health care and public health to better serve their communities in the future. The setting spans urban and rural environments, where animals provide food and income and are an integral component of household survival strategies. The rotation takes place in Meru University of Science and Technology, Kenya, and includes a one week didactic seminars with an emphasis on zoonotic diseases, environmental health and One Health concepts, followed by an intensive hands-on 3 week experience focusing on zoonotic pathogens and methods to assess and control human, animal and environmental contaminants. The students assess human-animal-environment interactions, develop group research projects and present the results to community leaders to address local challenges. Because of its potential to enhance inter-professional education, this rotation will be expanded to include other health professions at WUHS.

\section{Setting Research Priorities for Zoonoses Control: A Seven-Country Study}

\author{
Manish Kakkar ${ }^{1}$, Fabiola Quesada ${ }^{2}$, Artama Wayan Tunas ${ }^{3}$, Pranab Chatterjee ${ }^{1}$ and Severine Thys ${ }^{4}$ \\ ${ }^{1}$ Public Health Foundation of India \\ ${ }^{2}$ University of Pretoria \\ ${ }^{3}$ Universitas Gadjah Mada \\ ${ }^{4}$ Institute of Tropical Medicine
}

The recent outbreaks of zoonoses and emerging infectious diseases (ZEID), and the resulting global panic, emphasizes that they have become a global concern. Africa and South Asia are hotspots for ZEID risks. In order to understand the knowledge gaps, identify cross-country trends, and develop a global research agenda for zoonoses prevention and control, we deployed the modified CHNRI method in three African (Ghana, Ethiopia, South Africa) and three South Asian (Indonesia, Sri Lanka, Nepal) nations and a developed European country (Belgium), to serve as a comparator. At all sites, a wide range of experts are contributing to identification of priority ZEID issues and developing a strategic agenda in three steps: defining the contexts and identifying critical gaps; generating research options to address these gaps; and weighted scoring to establish a prioritized research agenda. Results from a study carried out on such a global scale is likely to produce insights that streamline the approach to ZEID risks. Emerging results show interesting patterns: for example, while there are some overlapping priorities, Indonesia has more concerns about outbreak prone diseases, while those in South Africa are 
centered around endemic zoonoses. By understanding the convergence and divergence of priorities, this study can help address the ZEID issues in a strategic, evidence-based manner.

\title{
Exploring Human Behaviors and Practices at High-Risk Interfaces for Disease Spillover in Sulawesi, Indonesia
}

\author{
Jusuf Daniel Kalengkongan ${ }^{1}$, dr(Vet) Joko Pamungkas $\mathrm{MSc}^{2}$, Kevin J. Olival $\mathrm{PhD}^{1}$ and Maureen Miller $\mathrm{PhD}^{1}$ \\ ${ }^{1}$ EcoHealth Alliance \\ ${ }^{2} I P B$ Bogor
}

High levels of undiagnosed disease occur in Sulawesi, Indonesia, along with potential behaviors (wildlife consumption) that may be high risk for zoonotic disease spillover. Little is known about the disease experiences and wildlife exposures among people involved in the wildlife trade. We conducted 47 targeted ethnographic interviews with people involved in the wildlife trade in Northern Sulawesi between February and July 2016. Participants included 26 women and 21 men, between 30 to 65 years old, and included hunters, collectors, transporters, vendors and consumers of wildlife. Half of the participants raised domestic animals and had free access to wildlife meat. No one reported using any protective gear when working with wildlife, (e.g., hunters used leaves and plants to carry captured wildlife). Hunters and vendors considered that they had no alternative livelihood options. The majority of participants were unaware of disease risks posed by wildlife, although $80 \%$ had experienced high fevers and other unusual illnesses. Local doctors attributed the illness to viruses of unknown origin. Herbs and traditional practices are used to cure such fevers. The lack of knowledge of disease risks posed by wildlife may facilitate zoonotic disease spillover.

639

\section{Working Towards Attainment of Effective Rabies Control in Zambia}

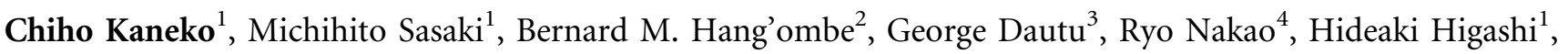
Chikako Kataoka ${ }^{1}$, Ryosuke Omori ${ }^{1}$, Hirofumi Sawa ${ }^{1}$, Ayato Takada ${ }^{1}$, Norikazu Isoda ${ }^{1}$, Aaron S. Mweene, Chihiro Sugimoto

${ }^{1}$ Research Center for Zoonosis Control, Hokkaido University

${ }^{2}$ School of Veterinary Medicine, The University of Zambia

${ }^{3}$ Ministry of Fisheries and Livestock, Zambia

${ }^{4}$ Graduate School of Veterinary Medicine, Hokkaido University

Periodic outbreaks of rabies occur in animals and humans in Zambia. Rabies control programme in Zambia relies on the vaccination of dog populations using domestically produced rabies vaccine which is used mainly in rural areas. However, the efficacy and the field effectiveness of the local vaccine are not elucidated. To estimate the vaccine coverage against rabies in dog populations, we conducted epidemiological surveys in urban $(n=200)$ and rural areas $(n=859)$ in Zambia. The efficacy and effectiveness of the domestic vaccine were evaluated and compared to those of the imported commercial rabies vaccine (Rabisin ${ }^{\circledR}$, Merial, France). The vaccine coverage in domestic dogs in urban area was $24.5 \%$ (95\% confidence interval: 18.5-30.5). The vaccine coverage in domestic dogs in rural area was attained at $42.4 \%$ (95\% confidence interval: 39.1-45.7) following the mass vaccination campaign. The proportion of the dogs which were successfully immunized with Rabisin ${ }^{\circledR}$ single shot was $76.9 \%$ (95\% confidence interval: 46.2-95.0), on the contrary, that with the local vaccine single shot was $12.5 \%$ (95\% confidence interval: 1.55-38.3). For the effective rabies control, studies to better understand the appropriate vaccination strategies are ongoing. 


\title{
Assessing and Understanding Food Safety Risk Practices in Nairobi Pork Food System: A Value Chain Approach
}

\author{
Maurice Karani ${ }^{1}$, Muinde Patrick ${ }^{1}$, Rushton Jonathan ${ }^{2}$, James Akoko ${ }^{1}$, Eric Fevre ${ }^{1,3}$, Dominguez Salas ${ }^{2}$, Dishon \\ Muloi $^{4}$, Barbara Haesler ${ }^{2}$ and Pablo Alarcon ${ }^{2}$ \\ ${ }^{1}$ International Livestock Research Institute \\ ${ }^{2}$ Royal Veterinary College \\ ${ }^{3}$ University of Liverpool \\ ${ }^{4}$ Edinburgh University
}

In Nairobi the pork food system may represent a major source of zoonotic pathogens. Yet, the system and its public health risks have not been described. The study address this gap by identifying and understanding food safety risks practices in the Nairobi pork food system using a value chain approach. A cross-sectional study of the Nairobi pork system collected data through focus group discussions and individual interviews with farmers, traders, abattoir owners, large companies' managers, retailers, government officers and consumers. Data were analysed to identify, describe and quantify the main pork chains, their food safety risk practices and explore their link with chain governance, distribution of benefits and barriers to improving the system. Main food safety risk practices for 'city pig keepers' were: handling and consumption of sick pigs. Pigs in slums were channelled directly to consumers and butchers or through less integrated markets (LIA). In 'LIA', risk practices were: lack of traceability and cold chain. These, with chains of pork from on-farm slaughtering, governed the lower end market. Among retailers, there was a lack of hygiene due to poor infrastructure and scarcity of water. The understanding obtained on chain governance, barriers and system inequalities associated to these risks provide a foundation to design control strategies.

920

\section{Expanding Constituencies for One Health}

\section{William Karesh}

EcoHealth Alliance, New York

In remarkable ways, the embracing of the One Health concept over the last decade has resulted in a dramatic shift in the discussions, practices, polices and partnerships that link the health of people, animals and our shared environments. In part, One Health has benefited from many innovative, collaborative efforts well underway and years in the making. Institutions large and small have found ways to utilize the theme of One Health, though the uptake has been strongest by the animal health and zoonotic disease community. While in itself this evolution has had positive effects, a question remains as to the value of expanding stakeholder engagement in One Health and where opportunities may lie. The principles of One Health are aligned with goals of developing global initiatives with different missions or visions but provide opportunities for highlevel political support. These include the Global Health Security Agenda, the Sustainable Development Goals, Future Earth, the Convention on Biodiversity's partnership with the World Health Organization, and World Bank Group's development initiatives. Thematically, these initiatives recognize that health underpins security and development, and in turn, are dependent on broader approaches to challenges such as climate change, improving resource and land-use decision making, food security, and biodiversity loss. 


\title{
Air Pollution and Respiratory Health in West Africa: Knowledge and Practices of Populations
}

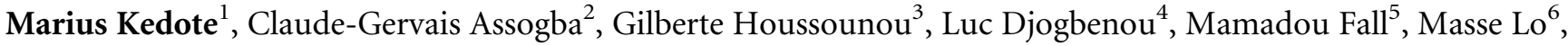
Issaka Tiembre ${ }^{7}$, Yveline Houenou ${ }^{8}$, Abdourahamane Konare ${ }^{9}$, Sandrine Lompo ${ }^{10}$, Karel Houessionon ${ }^{4}$ and Benjamin Fayomi ${ }^{11}$

${ }^{1}$ Ecohealth chair of COPES-AOC

${ }^{2}$ Ecole Nationale d'Aménagement et Gestion des Aires Protégés/Université de Parakou

${ }^{3}$ Consultante/Socio-anthropologie

${ }^{4}$ Institut Régional de Santé Publique/COPES-AOC/Université d'Abomey-Calavi

${ }^{5}$ Laboratoire de Toxicologie et Hydrologie/Université Cheikh Anta Diop de Dakar

${ }^{6}$ Consultant indépendant/Sociologie

${ }^{7}$ Faculté de médecine/Université Félix Houphouët Boigny d'Abidjan

${ }^{8}$ COPES-AOC/Université Félix Houphouët Boigny d'Abidjan

${ }^{9}$ Laboratoire de Physique de l'Atmosphère/Université Félix Houphouët Boigny d'Abidjan

${ }^{10}$ Santé au travail/Université de Ouagadougou

${ }^{11}$ Unité d'enseignement et de recherche de Santé au Travail et Environnement/COPES-AOC, Université d'Abomey-Calavi

In the aim to fight against air pollution, an intervention at community level termed "AIR-SAIN" is planned to be implemented in four West African cities. To assess the effectiveness of an intervention in human population, it is more adequate to perform a pre-intervention study to establish a baseline data, which will be used to evaluate the effect of the intervention.

The main objective of our study was to investigate the population (household) knowledge and behavior related to air pollution before the implementation of "AIR-SAIN" program.

The survey was conducted during the first semester of year 2016 based on a validated questionnaire, which were administrated to 959 households in eight districts from four cities (240 in Abidjan, 248 in Cotonou, 231 in Dakar and 240 in Ouagadougou).

The data collected during the survey were focused mainly on the characteristics of population's houses, their kitchens, their knowledge and practices related to air pollution in general, their beliefs about indoor pollution and their used of health services in the case of respiratory illnesses.

This study contributed to the planning of better activities to prevent air pollution in general and especially indoor air pollution based on real population's needs.

\section{The Ripple Effect: Understanding Culture and Responding to Suicide Stigma in Australian Farmers}

\author{
Alison Kennedy and Susan Brumby \\ Deakin University/National Centre for Farmer Health
}

Australian farmers live and work in cultural and social conditions that affect their wellbeing, and their ability to access appropriate support services. Farming men have an increased risk of suicide and are exposed to the suicidality of others. Self-stigma arising from an experience of suicide is common. Stigma - actual or perceived - can lead to feelings of isolation, weakness, shame, a sense of rejection, an ongoing risk of suicide, and poor health outcomes. 
Validated survey tools - the Stigma of Suicide Scale (SOSS) and the Literacy of Suicide Scale (LOSS) - will be used pre and post intervention to measure changes in stigma and literacy. The target population is 473 farming community males aged 30-64 years with an experience of suicide. The digital intervention comprises 5 chapters comprising digital stories; means for telling their own story; personal goal setting; information about suicide, stigma, health and wellbeing; and, links to support. The website will launch June 30th 2016 and run until January 2017.

This presentation will discuss the first wave of participants and early analysis.

This digital intervention recognises the social and cultural context of farming life and work, particularly farmers' willingness to help others while often avoiding seeking help themselves.

765

\title{
Hantavirus Risk in an Ecosystem Context
}

Hussein Khalil, Frauke Ecke, Gert Olsson, Magnus Evander, Magnus Magnusson, Birger Hörnfeldt

Hantaviruses are emerging infectious pathogens that are spread worldwide and cause disease in humans. Typically, Hantaviruses have one small mammal reservoir; and pathogenic Hantaviruses are often carried by generalist rodents which tolerate human-induced disturbance and persist at low diversity. Detailed ecological knowledge is required to assess and predict disease risk by identify "unhealthy" ecosystems (habitat patches) where the density of infected animals is highest. Both the density of host species and proportion of infected animals are important in determining risk, but may be influenced by different and opposing factors. For example, intermediate levels of fragmentation may result in the local extinction of competitors and predators but enable persistence of high density populations of host species in suitable habitat patches; whereas extreme levels of fragmentations may result in local extinction of the host. We here discuss how habitat type and disturbance, host predators, and competitors interact to determine disease risk and we rank these factors by their importance. Our synthesis is based on the ongoing project in northern Sweden through which multiple studies on Puumala hantavirus and its host have been conducted, and we discuss our results in relation to public health and nature conservation.

\section{4}

\section{Implications of Afghan Conflict on Trans-Boundary Kuchi Nomads' Mobility, Disease Transfer and Livelihood}

\author{
Javed Khan ${ }^{1}$ and Inam Rahim ${ }^{2}$ \\ ${ }^{1}$ Relief International \\ ${ }^{2}$ FRESH Pakistan
}

There are about 1.4 million Kuchi (nomads) rearing more than 50\% livestock of Afghanistan. A sizable portion of these nomads used to move with their herds from the central Afghanistan summer highlands to the Pakistani Indus valley plains wintering lowlands. The past 35 years of conflict in Afghanistan has negatively impacted this trans-boundary mobility and Kuchi's livelihood. This paper reflects for the first time on the demographic make-up of the trans-boundary Kuchis and their livestock, including their migratory patterns and implications of the conflict for their livelihood and zoonoses. Methodology included transect walk across areas occupied by Kuchi during winter, group discussions and semi-structured interviews in Naran an Zhob. Our study shows that toward the North some 350 Kuchi families have shifted their mobility during summer from the central Afghan highlands to the Himalayan uplands of Naran in Northern Pakistan. Toward the south near Zhob, many Kuchi's still cross into Pakistan with their livestock herds. The traditional mobility across the border is hindered and access to summer highlands curtailed due to competing claims of the settled communities. The study reflects on the livestock dynamics, livestock disease pattern and access to services and recommends measures to improve access to services. 


\title{
Biosafety \& Biosecurity Challenges in Pakistan
}

\author{
Muhammad Khan \\ National TB Control Program, Islamabad, Pakistan
}

Research with infectious agents is critical for the development and availability of public health and medical tools that are needed to detect, diagnose, recognize, and respond to outbreaks of infectious disease of both natural and deliberate origin. The expansion of infrastructure and resources dedicated to work with infectious agents has raised concerns regarding global preparedness with respect to Biosafety and Biosecurity. In this context Pakistan finds itself in a precarious position as evidenced by the recent Ebola/Zika outbreaks. While there is limited capacity for addressing Biosafety and Biosecurity in the country per se, these paradigms have scaled some success in the shape of specific vertical programs. The Multi Drug Resistant intervention of the National TB Control Program is in the process of establishing Biocontainment facilities-BSL 2/3 - which meet the desired international requirements for Biosafety \& Biosecurity. The process of establishing these facilities reveal limited understanding of the need of such sites in Pakistan and expertise to enable the same. This warrants government, academic and private sector initiatives to incorporate the complete biorisk management systems. Such initiatives would promote Biosafety and Biosecurity leading to improved safety of those who work within laboratories and the communities that host these facilities.

\section{4}

\section{Epidemiology and Laboratory Collaboration and Information Sharing: One Health Approach}

\section{Oanh Kim}

Epidemiology and laboratory collaboration between the human health and animal health sectors is a fundamental requirement and basis for an effective One Health response to the challenge of emerging infectious diseases in Viet Nam. During the past decade, there has been significant investment in laboratory equipment and training as well as support for quality assurance and improved networking, covering both the human and animal health laboratory systems with a focus on both diagnostic and research capacity related to human, livestock and wildlife samples. There has also been extensive investment in enhanced active and passive surveillance systems as well as epidemiological capacity in both the human and animal health sectors over the same period. However, internal and external assessments and evaluations within both sectors indicate the persistence of specific gaps in the implementation of Joint Circular 16 on coordinated prevention and control of zoonotic diseases, information sharing and inter-sectoral collaboration. It is needed for clear, structured and practical guidance as well as strong commitments from Human Health-Animal Health sectors in strengthening epidemiology and laboratory collaboration and information sharing, based on relevant policies and regulations as well as actual practices.

\section{7}

\section{Climate Change and Vector-borne Diseases: Use of Participatory Epidemiology to Investigate Experience in Vulnerable, Cattle-keeping Pastoral Communities of Monduli District, Northern Tanzania}

\author{
Esther Kimaro, Jenny-Ann Toribio and Siobhan Mor \\ University of Sydney, Australia
}

Climate change is predicted to increase incidence of vector-borne diseases (VBDs) in humans, however little is known about its impacts on livestock. Participatory epidemiological (PE) methods were used with Maasai pastoralists to establish local 
observations on East Coast Fever (ECF) and Animal Trypanasomiasis (AT). Data were collected between November 2014 and January 2015 in ten randomly selected villages involving gender segregated groups. Matrix scoring confirmed that Masaai easily recognise ECF and AT, and that these diseases ranked amongst the top five most important cattle diseases (strong agreement between informant groups; Kendall's $\mathrm{W}=0.399$ for men and 0.451 for women; $\mathrm{p}<0.01$ ). All groups associated ECF with the wet season while AT was more variable throughout the year, with more cases reported in dry seasons. Likewise, different villages reported seasonal differences in occurrence of Rhipicephalus appendiculutus and Tsetse flies. Comparing 2014 to 1984, participant groups consistently reported declines in rainfall, vegetation cover, quality pasture, and increases in severe droughts. Experiences with ECF/AT and vector abundance were more variable across villages. Preliminary analysis reveals a complex interplay between human, animal and environmental factors, understanding of which is urgently required to devise approaches to mitigate effects of climate change in vulnerable areas.

178

\title{
Bringing Together Ecohealth and One Health to Address Zoonotic Disease: Participatory System Dynamics Modelling as a Convergence Methodology
}

\author{
Lucy Kivuti ${ }^{1}$, Alex Macmillan ${ }^{2}$ and Patricia Priest ${ }^{2}$ \\ ${ }^{1}$ University of Otago/University of Nairobi \\ ${ }^{2}$ University of Otago
}

We explored One health and EcoHealth as approaches to addressinginfectious disease, and whether participatory system dynamics (PSD) modelling holds promise as a convergence methodology. Taking zoonotic disease as a case study, we performed a systematic review to identify epistemological and methodological differences between EcoHealth and One Health approaches in both theory and practice. We searched Google Scholar, PubMed, Web of Science, and Scopus for papers published between 2011 and 2016, using the terms 'EcoHealth', 'OneHealth', 'ecosystems', and 'zoonotic'. We drew out epistemological and methodological themes and identified overlaps and difference between the two approaches and considered the extent to which SD modelling brings together the strengths of both. SD enables understanding of non-linear relationships in complex systems, emphasising feedback loops and changing relationships over time. Multiple perspectives can be brought together in causal loop diagrams, which canbe translated into a SD simulation model to test policy options. When combined with a robust collaborative learning process, PSD couldsupport policy formulation for zoonosis reduction, building on robust data in a One Health framework while incorporating the participatory, holistic principles of Ecohealth.

416

\section{Pneumonia Risk for People Living Close to Intensive Animal Farms - Taking GPS Derived Mobility Patterns into Account}

Gijs Klous ${ }^{1}$, Lidwien A.M. Smit ${ }^{2}$, Gudrun S. Freidl ${ }^{3}$, Floor Borlée ${ }^{4}$, Wim van der Hoek ${ }^{5}$, Mirjam E.E. Kretzschmar $^{6}$, Dick J.J. Heederik ${ }^{2}$, Roel A. Coutinho ${ }^{7}$ and Anke Huss ${ }^{2}$

${ }^{1}$ Julius Centre for Health Sciences and Primary Care, University Medical Centre Utrecht, the Netherlands/Institute for Risk Assessment Sciences, division Environmental Epidemiology, Utrecht University, the Netherlands

${ }^{2}$ Institute for Risk Assessment Sciences, IRAS, Utrecht University, the Netherlands 
${ }^{3}$ Centre for Infectious Disease Control, National Institute for Public Health and the Environment (RIVM), Bilthoven, the Netherlands/European Programme for Intervention Epidemiology Training (EPIET), European Centre for Disease Prevention and Control (ECDC), Stockholm, Sweden

${ }^{4}$ Institute for Risk Assessment Sciences, IRAS, Utrecht University, the Netherlands, Netherlands Institute for Health Services Research, NIVEL, Utrecht, the Netherlands

${ }^{5}$ Centre for Infectious Disease Control, National Institute for Public Health and the Environment (RIVM), Bilthoven, the Netherlands

${ }^{6} J u l i u s$ Centre for Health Sciences and Primary Care, University Medical Centre Utrecht, the Netherlands/National Institute for Public Health and the Environment (RIVM), Bilthoven, the Netherlands

${ }^{7}$ Julius Centre for Health Sciences and Primary Care, University Medical Centre Utrecht, the Netherlands/Faculty of veterinary medicine, Utrecht University, the Netherlands

We previously observed an increased incidence of pneumonia in persons living near goat farms, using animal presence around the home to define exposure. However, it is unclear to what extent individual mobility contributes to this increased risk. We aimed at evaluating pneumonia-risk by assessing mobility patterns, considering exposure to goat farms based on movement patterns, compared to exposure assessment based on home address. In a rural Dutch cohort, 667 members logged their mobility using GPS trackers for 7 days. Pneumonia was diagnosed in 68 subjects (self-reported and general practitioners-data, 2011-2014). We used logistic regression to evaluate pneumonia-risk by presence of goat farms within $1000 \mathrm{~m}$ around the home and around GPS-tracks (only non-motorised mobility) or in standard deviation (SD) ellipses (spatially generalized areas around GPS-tracks corresponding to areas where people spend most time). Presence of goats within $1000 \mathrm{~m}$ of homes was associated with pneumonia (OR 2.7 (95\% CI 1.5-4.7)). For buffers around actual tracks, the OR was 3.5 (1.1-11.3), for 1-SD ellipse it was 1.6 (0.9-2.9), and for 2SDs, the OR was $2.8(1.2-6.5)$, with $\mathrm{N}=22, \mathrm{~N}=65, \mathrm{~N}=50$ and $\mathrm{N}=62$ exposed cases, respectively. We will further evaluate whether using mobility patterns instead of just the home address may improve exposure assessment.

805

\title{
Resilience of Populations to Malaria and Schistosomiasis in the Context of Climate Change in West African Sahel (Côte D’ivoire, Mauritania)
}

\author{
Amoin Jeanne d'Arc Koffi ${ }^{1}$, Gilbert Fokou ${ }^{2}$, N’Doumy Noel Abé ${ }^{3}$, Brama Koné ${ }^{4}$ and Bonfoh Bassirou ${ }^{2}$ \\ ${ }^{1}$ University Alassane ouattara, bouaké; Centre Suisse de Recherches Scientifiques, Abidjan, Côte d'Ivoire \\ ${ }^{2}$ Centre Suisse de Recherches Scientifiques, Abidjan, Côte d'Ivoire \\ ${ }^{3}$ Université Alassane ouattara, Bouaké, Côte d'Ivoire \\ ${ }^{4}$ Centre Suisse de Recherches Scientifiques; University Péléforo Gon Coulibaly, Korhogo
}

Malaria and schistosomiasis are still important public health concerns in sub-Saharan Africa, such as Côte d'Ivoire and Mauritania., in spite of tremendous efforts from researchers and policymakers for their control. Those figures are aggravated by the process of climate change that contributes to change relationships between human beings and their environment.. This study aims to analyze proactive and reactive mechanisms of populations to overcome the burden of malaria and schistosomiasis. Ecohealth methodology was implemented. This consisted of two cross-sectional household surveys in rainy and dry seasons in each site. Quantitative data, questionnaire ( $\mathrm{n}=728$ in Korhogo and 721 in Kaèdi) per season. Qualitative data: techniques of photovoice $(\mathrm{n}=80)$ and focus group $(\mathrm{n}=64)$ with communities members. From the preliminary results, it appears that populations rely on various strategies of resilience ranging from individual to institutional strategies. The use of self-medication and natural plants in both locations shows that people rely basically on individual resources. Social resources are also mobilized through family and neighborhood networks. 
However they are not clearly oriented to health problems. Support from structures do not appear to be a major asset for resilience. Conclusively, people in both sites have not yet developed cost-effective resilience mechanisms to malaria and schistosomiasis.

810

\section{Crimean Congo Haemorrhagic Fever, 2014 Sudan}

Claudia Kohl ${ }^{1}$, Therese Muzeniek ${ }^{1}$, Mawahib Eldegail ${ }^{2}$, Iman Mahmoud ${ }^{2}$, Annika Brinkmann ${ }^{1}$, Piotr Wojtek Dabrowski ${ }^{1}$, Livia Schrick ${ }^{1}$, Aleksandar Radonic ${ }^{1}$, Petra Emmerich ${ }^{3}$, Toni Rieger ${ }^{3}$, Stephan Günther ${ }^{3}$, Andreas Nitsche $^{1}$ and Abdalla Abdelkarim Osman ${ }^{2}$

${ }^{1}$ Robert Koch Institute

${ }^{2}$ National Public Health Institute

${ }^{3}$ Bernhard-Nocht-Institute for Tropical Medicine

In 2014 an outbreak of hemorrhagic fever in humans was reported from different states of Sudan. The NPHL, Khartoum investigated the cases and forwarded 28 sera samples from patients suffering from hemorrhagic fever to the RKI. The sample-set included a panel of 10 sera collected during former hemorrhagic fever outbreaks in the same region in 2013. All sera were tested with qPCR assays for Filoviruses and CCHFV. Additionally, all samples were subjected to metagenomic deep sequencing on an Illumina MiSeq sequencer.

CCHF was identified by two independent qPCR assays in a sample from November 2013 and November 2014, respectively. Deep sequencing confirmed these results. Based on the available sequences the novel CCHFV strain 'Sudan 2014' shares 96\% identity (na) with its closest relative CCHFV SPU 187/90 from South Africa.

CCHFV is reported to be transmitted by ticks in Europe, Asia and Africa and known as etiological agent of severe hemorrhagic fever in humans and livestock. Beside insect-repellent no preventive measures are available. The pathogenicity and characteristics of this novel strain have yet to be determined by cell-culture isolation and serology. Further molecular analysis will contribute to clarify the divergence of the CCHFV strains detected in 2013 and 2014. First results including novel viruses identified by metagenomics will be presented.

\section{Integrative Approach to the Control of Malaria and Schistosomiasis in the Northern and Southern Fringes of the Sahelian Belt in a Context of Climate Change}

Brama Kone ${ }^{1}$, Mouhamadou Chouaïbou ${ }^{2}$, Sid'Ahmed Dahdi ${ }^{3}$, Dieudonné K. Silue ${ }^{4}$, Emmanuel L.J-C. Esso ${ }^{4}$, Yves N. Tian-Bi ${ }^{4}$, Gilbert Fokou ${ }^{2}$, Hampaté Bâ ${ }^{5}$, Moussa Keita ${ }^{3}$, Ousmane Bâ ${ }^{3}$, Ibrahima Sy ${ }^{6}$, Gregoire Y. Yapi ${ }^{7}$, Emmanuel Tia ${ }^{7}$, Mohamed Doumbia ${ }^{4}$, Tanoh A.S.R. Nkrumah ${ }^{4}$, Constant Gbalegba ${ }^{8}$, Richard K. Mbra ${ }^{4}$, Jeanned'Arc Koffi ${ }^{4}$, Aboudramane Kaba ${ }^{4}$, Honorate Ballé ${ }^{4}$, Dramane Soro ${ }^{4}$, Moussokoro Sidibé $^{3}$, Cheikh M. M. Seyed ${ }^{3}$, Giovanna Raso ${ }^{9}$ and Benjamin G. Koudou ${ }^{10}$

${ }^{1}$ University Peleforo Gon Coulibaly, Korhogo \& Centre Suisse de Recherches Scientifiques, Abidjan

${ }^{2}$ Centre Suisse de Recherches Scientifiques, Abidjan

${ }^{3}$ University of Sciences and Technologies of Medicine, Nouakchott

${ }^{4}$ University Félix Houphouet Boigny \& Centre Suisse de Recherches Scientifiques, Abidjan

${ }^{5}$ Institut National de Recherches en Santé Publique, Nouakchott

${ }^{6}$ Centre de Suivi Ecologique, Dakar

${ }^{7}$ Université Alassane Ouattara de Bouaké 


\footnotetext{
${ }^{8}$ University Nangui Abrogoua \& Centre Suisse de Recherches Scientifiques, Abidjan

${ }^{9}$ Swiss Tropical and Public Health Institute, Basel

${ }^{10}$ Lyverpool School of Tropical Medecine
}

Ecohealth methodology was implemented from 2013 to 2016 in two cities of the Sahel belt to understand the complexity of hazards, vulnerabilities and exposures to malaria and schistosomiasis and identify sustainable solutions for resilience. In each city, following a multi-stakeholder' engagement process, two cross-sectional surveys were done in dry and rainy seasons, namely, household questionnaire, blood, feces and urine analysis, entomological, malacological and geographical surveys. Additionally, weather and climate data were generated and analysed. In Korhogo (Côte d'Ivoire), intestinal schistosomiasis is predominant (4.6\%) while urinary forms predominate in Kaedi $(4 \%)$ and are statistically higher in the dry season $(\chi 2=5.64$; $\mathrm{p}=0.017)$. Malaria prevalence was $12.5 \%$ and $0.3 \%$ respectively in Korhogo and Kaedi with a predominance of $P$. falciparum. Dissolved oxygen has a positive significant correlation with the presence of Anopheles gambiae larvae $(\mathrm{OR}=1.20 ; \mathrm{p}=0.029)$. Rainfall of the preceding two months was associated to an increase of malaria incidence of 1\%. The most important assets of communities to face the diseases are the individual and social capitals. Results allowed the identification of capacity building activities for community resilience. Preliminary results are proving usefulness of Ecohealth methodology for a sustainable adaptation of malaria and schistosomiasis-affected communities to climate change and/or variability.

\title{
1064
}

\section{Environmental Conditions and Biological Profile of Patients with Major Depressive Disorder With or Without Suicidal behavior}

\author{
Edouard Kouassi ${ }^{1,2,3}$, Réal Labelle ${ }^{2,3,4}$, Charles-Edouard Giguère ${ }^{2,3}$ and Sonia Lupien ${ }^{1,2,3}$ \\ ${ }^{1}$ Faculty of medicine, Université de Montréal \\ ${ }^{2}$ Institut universitaire en santé mentale de Montréal \\ ${ }^{3}$ The Signature Consortium \\ ${ }^{4}$ Université du Québec à Montréal (UQAM)
}

This study characterized the environmental conditions and biological profile of patients with major depressive disorder (MDD) and suicidal behavior (SB) who are admitted in the psychiatric emergency room. The study is based on the Signature Bank, a biopsychosocial bank collected and stored from people with mental health problems. The bank is located in Canada and it is accessible to researchers around the world. The first 50 patients with MDD with or without SB were selected (28 men and 22 women). Their mean age was 43-y (SD, 13-y). Their education level and annual earnings were lower, while they reported more adverse childhood experiences in comparison with a matching control group of 50 non-hospitalized individuals from the 2012 Canadian Community Health Survey on Mental Health. Plasma levels of the inflammatory cytokine tumor necrosis factoralpha (TNF-a) were positively and significantly correlated with suicidal ideations. These findings contribute to better characterize the poor socioeconomic conditions of life of MDD patients admitted in the psychiatric emergency room. Additionally, the data suggest that plasma TNF-a may serve as a susceptibility marker of suicidal risk in patients with MDD. Novel strategies are likely to emerge in order to predict and prevent suicidal risk in MDD patients.

633

\section{One Health Approaches in Dealing with Salmonella and Campylobacter in Queensland}

\author{
Nina Kung ${ }^{1}$, Andrew Wilson ${ }^{2}$ and Anna Sartor ${ }^{3}$ \\ ${ }^{1}$ Biosecurity Queensland, Department of Agriculture and Fisheries \\ ${ }^{2}$ Safe Food Production Queensland \\ ${ }^{3}$ Queensland Health
}


The food-pathogen combinations of Campylobacter-poultry meat, and Salmonella-eggs are frequently associated with foodborne illness. Poultry production facilities, poultry meat, eggs and egg products can easily become contaminated either during primary production, processing, or further along the supply chain. High consumption rates and growing demand for poultry products also increase the risk of exposure by Salmonella and Campylobacter to consumers. In Queensland, the reduction of foodborne illness is a priority and is achieved through a legislative framework which is administrated by three state government agencies - Queensland Health, Safe Food Production Queensland (SFPQ) and Department of Agriculture and Fisheries (DAF) to address food safety at different levels of the food supply chain. The three agencies have developed a strategy to facilitate engagement between Queensland food regulators and the food industry in order to ensure resources are focused on appropriate action along the food supply chain to control food safety risks. These objectives are achieved through the implementation of coordinated strategies, including the Queensland Foodborne Pathogen Risk Reduction Strategy and the formation of a Science Group for a co-ordinated one health, supply chain approach in controlling foodborne pathogens in Queensland.

\title{
Downstream Effects of Globalization and Agricultural Policy on Human Risks to Scrub Typhus and Tick-Borne Diseases
}

\author{
Chi-Chien Kuo, Jing-Lun Huang, Pei-Yun Shu, Pei-Lung Lee, Douglas Kelt and Hsi-Chieh Wang \\ National Taiwan Normal University
}

Globalization can lead to abandonment of farms in countries with higher costs of agricultural practice, and may have important implications for human health. We evaluated how extensive desertion of rice paddies in Taiwan after joining World Trade Organization, and how periodic plow, an agricultural policy targeting for farm pests, can unexpectedly influence ticks and larval trombiculid mites (chiggers). Flooded rice fields were assumed to sustain very few ticks and chiggers. Striped field mice, main hosts for both vectors, harbored 6 times more ticks, and 3 times more chiggers in abandoned (fallow) than in plowed plots. The proportion of ticks infected with Rickettsia spp. (etiologic agent of spotted fever) was $3 x$ higher in fallow plots, while that of Orientia tsutsugamushi (scrub typhus) in chiggers was similar in both treatments. Moreover, ticks and chiggers were dominated by species known to infest humans. We conclude that ticks and chiggers proliferate after rice paddies are abandoned, but periodic plowing may mitigate vector burdens. An unexpected consequence of globalization may be elevated disease risk in the face of economically-induced changes in agricultural practices, although this may be unintentionally mitigated by agricultural policies, calling for further research on vector-borne diseases and their control from broader perspectives.

\section{8}

\section{A Modified Time-Series Regression Approach to Examine Non-Linear Associations of Climate Variability with Infectious Cryptosporidiosis Across Three Climate Zones in Australia}

\author{
Aparna Lal ${ }^{1}$, Simon Hales ${ }^{2}$ and Masahiro Hashizume ${ }^{3}$ \\ ${ }^{1}$ Australian National University \\ ${ }^{2}$ University of Otago \\ ${ }^{3}$ Nagasaki University
}

Associations of health outcomes with global indices of environmental change can provide important insights into the pathways through which such change may impact health, but previous illnesses can modify this time-varying relationship between environmental exposures and disease. To examine the influence of past illnesses, the non-linear and time varying association of cryptosporidiosis with an index of global climate variability, the Dipole Mode Index (DMI), was assessed using models 
modified to include past cryptosporidiosis counts. As exposure-disease relationships vary geographically, we focused on weekly reports of cryptosporidiosis from 2001 to 2012 in Australia from the tropical, subtropical and temperate regions (92\% of all reported cryptosporidiosis). In the subtropical region, disease risk was strongly positively associated with an immediate increase in DMI, although this general relationship was observed across all climate zones. These findings provide some support for the previously established role of rainfall with cryptosporidiosis as the expected increases in the frequency of positive DMI events may result in regional decreases in rainfall, increasing turbidity and pathogen concentration in water sources, leading to more waterborne cryptosporidiosis. Future models for environmentally sensitive infectious diseases that account for previous illness may better represent the time dependant environmental exposure-disease relationship.

682

\title{
Evaluation Capacity Building in an Integrative Research Organization
}

\author{
Steven Lam ${ }^{1}$, Phuc Pham-Duc ${ }^{1}$ and Hung Nguyen-Viet ${ }^{2}$ \\ ${ }^{1}$ Center for Public Health and Ecosystem Research, Hanoi School of Public Health, Hanoi, Vietnam \\ ${ }^{2}$ International Livestock Research Institute, Hanoi, Vietnam
}

Evaluation capacity building has gained traction in many organizations as they face increasing demands for accountability and sustainable evaluation practice. Its goal is to strengthen an organization's knowledge and skills in designing, implementing, and managing effective evaluation projects, and using evaluation findings for decision-making and action. While evaluation capacity building activities and processes vary in organizations, the strategies being used and the overall effectiveness are not well explored. Going further, building capacity in evaluation of integrative approach projects (e.g. Ecohealth projects) come with its own set of challenges. Evaluation capacity building of a new Ecohealth research center has been supported by volunteers from the Volunteers for Healthy Animals and Healthy communities program (Vets without Borders Canada). We present a framework, and discuss teaching and learning strategies used to facilitate capacity building (e.g. a combination of workshops and mentoring). Lessons learned and challenges will be shared, for example essential evaluation core capacities, and operational challenges of Ecohealth research projects. The authors conclude with their own learnings and reflections of building evaluation capacity.

\section{8}

\section{The Impact of Working Equids on the Social Determinants of Health: Evidence from Rural Nicaragua}

\section{Jennifer Lane \\ Congressional Hunger Center}

The importance of working equids (horses, donkeys, and mules) to economic survival and human livelihoods in poor and rural communities around the world is indisputable. There are approximately 112 million working equids in the developing world; in some countries populations are increasing. A strong case exists for the impact of working equids on the social, economic, and cultural determinants of health. Working equids are essential for goods transport and contribute to economies and food security locally and globally. Working equids may enable access to health services or social support networks. Working equid owners are often lower in the social gradient, under social and economic stress, and may be socially excluded. If a working equid is unable to perform, a child or woman may replace that labor, which can adversely affect childhood development. This may also translate into unemployment, lost income and time. Using evidence from a cross sectional survey of working equid owners in rural Nicaragua and the existing literature, the interdependence of livelihoods and health on working equids is illustrated. Understanding how livelihoods and health depend on a working equid's contributions to a household has broad reaching policy and development implications. 


\section{Human Leptospirosis in Fiji: An Eco-epidemiological Approach to Under- standing Zoonotic Disease Transmission and Informing Public Health Inter- ventions}

Colleen Lau ${ }^{1}$, Conall Watson ${ }^{2}$, John Lowry ${ }^{3}$, Michael David ${ }^{4}$, Sarah Wynwood ${ }^{5}$, Scott Craig, Mike Kama ${ }^{6}$ and Eric Nilles ${ }^{7}$

${ }^{1}$ Australian National University

${ }^{2}$ London School of Hygiene \& Tropical Medicine

${ }^{3}$ The University of South Pacific

${ }^{4}$ The University of Queensland

${ }^{5}$ Department of Health, Queensland

${ }^{6}$ Fiji Ministry of Health \& Medical Services

${ }^{7}$ World Health Organization

Leptospirosis is an emerging infectious disease in the Pacific Islands. Disease transmission is strongly driven by humansanimal-environment interactions, which in turn are mediated by broader socio-demographic, cultural and environmental factors. In Fiji, two successive cyclones and severe flooding in 2012 resulted in outbreaks associated with 7\% case-fatality. A field study was conducted in 2013 using an eco-epidemiological approach to integrate survey data with geospatial environmental and census data, and quantify the relative importance of risk factors at different ecological scales. Leptospira antibodies were found in $19.4 \%$ of 2152 participants from 81 communities on the 3 main islands. On multivariable logistic regression modelling, significant individual-level risk factors included male, ethnicity, poor access to water, and working outdoors. Significant environmental predictors of infection included living in rural areas (OR 1.43), poverty rate (OR 1.74 in communities with $\geq 40 \%$ poverty), living $\leq 100 \mathrm{~m}$ from a major river (OR 1.41), pigs in the community (OR 1.54), high cattle density in district (OR 1.04 per head/sqkm), and rainfall in wettest month (OR 1.003 per mm). Predictive accuracy of the model highlights that broad-scale environmental and socio-demographic factors play crucial roles in disease transmission, and the importance of an ecological approach to public health intervention strategies.

924

\section{Expanding Disease Control in South Africa Through the Rabies One Health Model}

\section{Kevin Le Roux}

Disease project manager, South African Veterinary Services KwaZulu-Natal

An international collaborative project to demonstrate the feasibility of eliminating human rabies in KwaZulu-Natal South Africa, has inadvertently left behind it a model for One Health in Developing countries. Historically rabies is probably the "original one health story", and the close multi-sectoral collaboration needed for control, combined with the need to understand the complex symbiotic relationship between humans and animals, has allowed for the formation of a One Health network in terms of research, advocacy and control, that can be generically applied to many zoonotic diseases.

This foundation is now being applied to Brucellosis and has effortlessly created a platform, for research and control that has been absent in South Africa for many years. The tendency to decentralize major disease control to local level, often results in reduced political backing, and collective disease control efforts. In addition fragmentation of effort can erode the advantages of specialisation, and champion driven programs. This One Health model brings individual diseases under specialist management, and ensures purposeful attainment of goals, while allowing existing networks to overlap, expand and complement each other. 


\title{
Justice is the Missing Link in One Health: A Mixed Methods Study in an urban city state
}

\author{
Zohar Lederman ${ }^{1}$, Tamra Lysaght ${ }^{1}$, Benjamin Capps $^{2}$, Michele Bailey ${ }^{1}$, David Bickford ${ }^{1}$, Richard Coker ${ }^{3}$ and \\ Paul Anantharajah Tambyah ${ }^{1}$ \\ ${ }^{1}$ National University of Singapore \\ ${ }^{2}$ Dalhousie University \\ ${ }^{3}$ London School of Hygiene and Tropical Medicine
}

Singapore is one of many countries to embrace the One Health $(\mathrm{OH})$ framework for the prevention and control of Emerging Zoonotic Diseases (EZD). We conducted a mixed methods study using 11 semi-structured interviews and a modified Delphi technique with a panel of 32 key opinion leaders in Singapore. Panellists rated conceptions of $\mathrm{OH}$ and priorities for EZD preparedness planning using a series of scenarios developed through the study. Interview data were analysed qualitatively using thematic analysis techniques. Panellists agreed that $\mathrm{OH}$ is a cross-disciplinary collaborative effort between the veterinary, medical, and ecological sciences and the relevant government agencies encompassing animal, human, and environmental health policy and research. Human health was the most important priority in EZD planning. However, qualitative analysis suggested that consideration of non-human animal health and welfare as well was very important, and that effective pandemic planning demands regional leadership and international cooperation. Findings suggest that affluent urban states, such as Singapore, need to take on greater regional responsibilities and leadership roles in managing EZDs, especially those with potential global health impacts. Findings also suggest that culling is not an ethically viable response to EZDs. EZD planning under an $\mathrm{OH}$ approach would benefit greatly from an ethical framework that accounts for wider considerations of justice.

578

\section{Watershed Diagnostics for Improved Adoption of Management Practices: Integrating Biophysical and Social Factors Across Urban and Agricultural Landscapes}

Paul Leisnham, Hubert Montas and Adel Shirmohammadi

University of Maryland

The Chesapeake Bay situated on east coast of the United States has undergone considerable water quality degradation over the past 60 years. There has been little improvement in water quality with the focus of previous research and intervention on technological or social components alone. We propose a shift away from discipline-specific research and intervention towards an integrative research, extension, education approach that embraces both biophysical and social dimensions of pollution transport and Best Management Practice (BMP). We describe an inter-disciplinary project that has developed next-generation GIS-based assistive tools that integrate biophysical and social factors to target pollution hot spots and prescribe appropriate BMPs in urban and agriculture watersheds. It used social research to evaluate stakeholder attitudes and behaviours towards watershed health and BMP adoption, and combined these results with biophysical research within a Diagnostic Decision Support System (DDSS) to strengthen the technical abilities of community, State, and Federal partners at precisely targeting effective BMPs. Ecosystem-orientated education programs and technology transfer were applied in cooperative partnership with community associations and State officials in study watersheds to improve effective outreach strategies and lower BMP adoption thresholds so that greater advancements and actions can be made towards watershed sustainability. 


\title{
Impacts of Invasive Urban Mosquitoes on Resident Species: Differences in the Outcome of Competition Among Habitats Affects Vector Populations
}

\author{
Paul Leisnham ${ }^{1}$ and Shannon L. LaDeau ${ }^{2}$ \\ ${ }^{1}$ University of Maryland \\ ${ }^{2}$ Cary Institute of Ecosystem Studies
}

Invasive species and infectious disease are signature topics in coupled natural-human systems research. But the social and ecological mechanisms underlying natural-human coupling can be complex and poorly understood. This talk will examine how the outcome of larval resource competition between disease-vector mosquitoes varies across habitats associated with socioeconomic and natural gradients in urban landscapes. As an example, this talk will focus on a study that tested whether competition from the competitively superior invasive mosquito, the Asian tiger mosquito, Aedes albopictus, on the resident mosquito, Culex pipiens, differed among managed containers from residential yards versus trash containers from re-wilding vacant lots in Baltimore, Maryland, USA. Collectively, Ae. albopictus and Cx. pipiens vector a range of arboviruses, and their distributions influence human disease risk. Field surveys showed a greater proportional decrease of Cx. pipiens in trash versus managed containers from early to late summer, when the densities of Ae. albopictus, and associated competition, increased. An accompanying laboratory experiment showed greater decreases in Cx. pipiens survival and development with increasing Ae. albopictus density in trash versus managed container treatments. The results of this study demonstrate how an ecological process, resource competition, is both influenced by human behaviour and affects human health.

\section{5}

\section{Attitudes in China Towards the Wildlife Trade}

\author{
Hongying Li ${ }^{1}$, Peter Daszak ${ }^{1}$, Maureen Miller ${ }^{1}$, Aleksei Chmura ${ }^{1}$, Grace $\mathrm{Ge}^{2}$, Guangjian Zhu \\ ${ }^{1}$ EcoHealth Alliance \\ ${ }^{2}$ International Fund for Animal Welfare
}

An e-survey was conducted among 2,238 Chinese nationals from Dec.15, 2015 to Jan. 15, 2016 to understand the behaviors and attitudes in China towards the wildlife trade. Although results showed an increased awareness of wildlife conservation (85\%), $72 \%$ of respondents were involved in wildlife trade, which was not significantly related to their education levels, geographical regions, or the cognition of wildlife and emerging infectious diseases. The main groups of consumers of wildlife in China are people who reported higher income aged 25-39 years old. There was very limited knowledge of the health risks posed by wildlife among respondents (28\%), which increases the risk of zoonotic disease transmission from animals to humans in China where several high profile zoonotic disease pandemics have emerged. Most respondents believed that not only is it imperative to stop wildlife trade (96.3\%), but that enforcement of policy by government would be the most effective way towards this aim (87\%). The utilitarian theory of wildlife resource and increasing per capita income in China are driving the wildlife trade, a One Health approach with collaborative efforts of multiple disciplines involving local communities is required in order to reduce the health and conservation impacts of wildlife trade in China.

923

\section{New Strategies for Controlling Cystic Echinococcosis and Taenia solium}

\section{Marshall Lightowlers \\ University of Melbourne}


Specific programs targeting human cysticercosis caused by Taenia solium and echinococcosis caused by Echinococcus granulosus have, in most cases, had limited outcomes or effects that were not sustained. Changes in endemic areas such as economic advancement, improvements in sanitation and education could be expected to reduce the prevalence of these diseases, however in many endemic areas these seem unlikely except in the distant future. Characteristics of the transmission cycles for cysticercosis and echinococcosis limit the opportunities that are available for combining interventions for these diseases together with other infections, including other NTDs. Vaccination of livestock animals to prevent transmission of T. solium and E. granulosus through their natural animal intermediate hosts is now a viable option for controlling both cysticercosis and cystic echinococcosis. Field trials of the TSOL18 and EG95 vaccines have demonstrated their effectiveness against field-derived infections in pigs and sheep, respectively. Critical to the further validation of these as control measures will be demonstration of the practicality and sustainability of their use in poor resource settings.

1088

\title{
Sero-Prevalence of Bovine and Human Brucellosis on Selected Farms in South-western Uganda
}

\author{
Nanfuka Mary Lovincer \\ Division of Disease Control, Ministry of Agriculture, Animal Industry and Fisheries
}

Brucellosis is among the zoonotic diseases that continue to afflict man and animals in Uganda. The increase in the number of Brucellosis outbreaks in animals from 1990 to 2013 and the numbers of human patients diagnosed with brucellosis in private clinics and hospitals has placed Brucellosis to be among the top re-emerging diseases in the country. Brucellosis infection in humans is non-specific and caused by direct or indirect contact with infected animals or their products. Brucellosis manifests as intermittent fever, headache, weakness, profuse sweating, chills, weight loss, generalized aching that may involve multiple organ systems in the body. In animals, Brucella organisms localize in the reproductive organs, causing abortions, decreased milk yields and temporary sterility. Its effects impact negatively to the sale value of the affected animals causing financial losses to the animal owners. Brucellosis was investigated in three districts of south Western Uganda by the staff of the Ministry of Agriculture, Animal Industry and Fisheries (MAAIF) in May and June, 2015 under the sponsorship of the United States Department of State Biosecurity Engagement Programme (BEP). Brucellosis test results from a total of 1503 cattle showed a sero-prevalence of $14 \%$ in Kiruhura, 18\% Bushenyi and 23\% Mbarara districts respectively. ELISApositive Brucella cases from farm attendants on the sampled farms in the same districts had a prevalence of $4 \%$ in Kiruhura, 9\% in Bushenyi and 12\% in Mbarara. This underscores Brucella exposure as one of the major re-emerging diseases that should be treated with great concern by both Ministry of Health and MAAIF. Based on our results, we believe that the Ministry of Health and Agriculture should increase on community sensitization on the risk of Brucella.infections in humans from cattle, and promote measures that can protect high risk families from getting infected. This survey indicates that Brucellosis infections are still prevalent in Uganda and continue to occur in the local communities. The public health and animal health service providers need to work together in compiling the disease epidemiological data for a concerted disease intervention measures.

1089

\section{Uganda a Hot Bed for Emerging Zoonotic Diseases: The Importance of Establishing Surveillance and Diagnostic Capacities}

\author{
Julius J. Lutwama
}

Uganda Virus Research Institute 
Uganda in the last couple of years experiences an average of 3 zoonotic diseases outbreaks every year. This makes it one of the most highly venerable countries for highly infectious disease outbreaks in Africa. There have been outbreaks of major zoonotic diseases such as Ebola, Marburg, Rift Valley Fever, Crimean Congo Hemorrhagic fever, Yellow Fever, Influenza, etc. While these are highly infectious diseases and may cause big outbreaks, they have been quickly detected and controlled in Uganda. Uganda has been able to detect and control these diseases without causing major outbreaks because of establishing capacity for surveillance and diagnostics, having a sample referral system, and continuous educaction and sensitization with support from CDC, GHSA and DTRA. Even some new infections like Sosuga virus, Sunguru virus, Bukakata virus, etc., have been identified through these systems.

\section{4}

\section{Zoonotic Arbovirus Monitoring in Victoria, Australia: Past, Present and Future}

Stacey Lynch, Jana Batovska ${ }^{1}$, Karen Brown ${ }^{1}$, Jodie Crowder ${ }^{1}$, Rebecca Feldman ${ }^{2}$, Mark Fegan ${ }^{1}$, Mark Blacket ${ }^{1}$ and Simone Warner

${ }^{1}$ Biosciences Research, Department of Economic Development, Jobs, Transport and Resources (DEDJTR)

${ }^{2}$ Department of Health \& Human Services, Regulation, Health Protection and Emergency Management Division

Vector monitoring, arbovirus detection and vertebrate sentinel surveillance form the foundations of an integrated surveillance program for zoonotic arboviruses. The Flaviviruses Murray Valley encephalitis virus (MVEV) and West Nile virus (subtype Kunjin) (KUNV) are members of the Japanese encephalitis virus serogroup. Both are endemic to Australia and are maintained in an enzootic cycle by wild birds and mosquitoes, with Culex annulirostris the principal bridge vector. Vector and arbovirus surveillance is conducted annually as part of an integrated arbovirus surveillance and control program. MVEV was last detected in Victoria in 2011, following a La Niña climatic event which led to extensive flooding throughout the state. Although there were no confirmed human cases in 2011, MVEV and KUNV (as well as Ross River virus) contributed to one of the largest equine arbovirus outbreaks in Australia. The magnitude of vector abundance and the co-circulation of arboviruses highlighted limitations with the methodologies currently employed within the surveillance program. Recently, advanced molecular methods, such as massive parallel sequencing, have the potential to improve the identification of mosquito species and virus isolates detected by surveillance programs. Such innovations will contribute to smarter surveillance programs, which are responsive to emerging endemic and exotic vector-borne risks.

\section{4}

\section{Climate Change Trends Potential Effects On Malaria And Schistosomiasis Transmission In Korhogo (Northern Côte D’ivoire)}

Kouassi Richard $\mathbf{M}^{\prime} \mathrm{Bra}^{1}$, Brama Koné ${ }^{2}$, Jacques André Ndione ${ }^{3}$, Ibrahima Sy ${ }^{3}$, Nagnin Soro ${ }^{4}$ and Guéladio Cissé ${ }^{5}$

${ }^{1}$ Centre Suisse de Recherches Scientifiques en Côte d'Ivoire (CSRS)

${ }^{2}$ Centre de Suivi Ecologique, Dakar, Senegal

${ }^{3}$ University Félix Houphouët Boiny, Abidjan, Côte d'Ivoire

${ }^{4}$ Swiss Tropical and Public Health Institute (Swiss TPH)

The aim of this study is to analyze potential links between past time-series data for climatic parameters and for malaria and schistosomiasis in Korhogo (Northern Côte d'Ivoire). Clinical malaria and schistosomiasis cases records were collected from health facilities over the decade 2005-2014. Rainfall, temperature and normalized difference vegetation index (NDVI) data over the same decade were obtained from the moderate resolution imaging spectroradiometer (MODIS) satellite 
images. Patterns of time series were explored by boxplots. The link between the variables was studied using quasi Poisson regression models. Each $1 \mathrm{~cm}$-increment in rainfall of two month before was on average associated with a $1.0 \%$ (95\%-CI: $0.4 \%$ to $1.6 \%$ ) increase in malaria incidence. Moreover, each 1 centigrade increment in average monthly temperature was on average associated with a change of $-4.2 \%$ (95\%-CI: -7.6 to $-0.7 \%)$ in malaria incidence. Also, a 0.1 unit increase in NDVI of three months was associated with a $-11.2 \%$ (95\%-CI: -21.59 to 0.6 ) decrease in monthly malaria count. $46.24 \%$ $\left(\mathrm{r}^{2}=0.46, \mathrm{p}=0.016\right)$ of monthly schistosomiasis cases was associated to the temperature of the 2 months before. Preparing appropriate adaptation plans to climate changes will be an important component in the development of strategies for increasing the resilience of communities.

1042

\title{
Antimicrobial Resistant Staphylococcus sp. in Dogs in Remote Australian Aboriginal Communities
}

\author{
Gemma Ma, Jacqueline Norris and Michael Ward
}

Community acquired methicillin-resistant Staphylcocccus aureus (MRSA) is a serious public health concern in Australian Aboriginal communities. Currently little is known about the epidemiology of these infections, however significant differences exist to hospital acquired MRSA infections for which risk factors have been well established. Dogs have been documented as asymptomatic carriers of MRSA in a number of studies despite this bacteria not being considered part of their normal flora. Dogs play an important role in Aboriginal communities with close inter-species contact. In addition dogs in remote communities are frequently overpopulated and have poor skin health. These factors make it likely that rates of MRSA carriage will be higher in dogs in Aboriginal communities than in other populations. A cross-sectional study is proposed for September 2016 to estimate the prevalence of asymptomatic MRSA carriage by dogs in 5 remote Aboriginal communities in NSW. Two hundred dogs will be recruited from a community dog health program. Swabs will be taken from the nares, buccal mucosa, perineum and skin lesions or wounds (if present) for bacterial culture. Antibiotic resistant isolates will undergo whole genome sequencing to determine their significance to human health. Full results will be available and ready for presentation by December 2016.

\section{8}

\section{Developing a Community Centered Early Warning System Based on Indigenous Knowledge: Gwanda District, Zimbabwe}

\author{
Margaret Macherera ${ }^{1}$ and Moses Chimbari ${ }^{2}$ \\ ${ }^{1}$ National University of Science and Technology/University of Kwazulu Natal \\ ${ }^{2}$ University of Kwazulu Natal
}

Malaria continues to be a major public health problem in Sub Saharan Africa despite efforts that have been made to prevent and control the disease. The knowledge on prediction of occurrence of the disease that communities acquired over the years has not been seriously considered in control programmes. This paper reports on studies that aimed to integrate indigenous knowledge systems (IKS) on malaria into the malaria control programme in Gwanda District, Zimbabwe. Data was collected using participatory rural appraisals, key informant interviews and workshops in 3 wards (11, 15 and18) with the highest malaria incidence in Gwanda district. Disease livelihoods calendars produced by the community showed their knowledge on the relationship between malaria and rainfall. Indigenous environmental indicators for the occurrence of malaria were classified as insects, plant phenology, animals, weather and cosmological indicators. Plant phenology was mentioned as the most important indicator. A community based malaria early warning system model was developed using 
the identified IKS indicators. In the model data on indicators was collected at village level by IKS experts, analysed at ward level by IKS experts and health workers and relayed to the District Health Team.

943

\title{
Zoonotic Disease, Biodiversity and the Community
}

\author{
Lenore Manderson \\ University of the Witwatersrand, South Africa
}

A series of ecological, economic and demographic changes are impacting on the distribution and endemicity of zoonotic diseases in resource-poor settings. The first is changes in climate, including in ambient temperature and precipitation, with impact on animal habitat, distribution and density. This has resulted in changes to host-feeding choices, human-vector contact rates and parasite transmission. These changes combine with changed vector habitat and behaviour in response to the use of insecticides, and changes in the density and residence patterns of humans and domesticated animals. In elaborating this, I consider three zoonotic diseases of poverty. In the case of Chagas disease, in South America, I illustrate how triatomin bugs vary their feeding patterns and so the transmission of T. cruzi depending on the location of domesticated animals. While improved human living conditions will reduce the potential for the bugs to infest human dwellings, this will also reduce the probability of human/animal co-residence, so reducing alternative blood meals for the bugs. I then consider African trypanosomiasis (sleeping sickness), which is recurring as human populations lose prior immunity, with a risk too of concentration in domesticated animal hosts as wild host populations decline. My third example, zoonotic schistosomiasis, highlights the complexities of ecosystems where humans and animals coexist, both in Asia and in Africa. In these examples, there is clear evidence of links between shifts in the density and regional settlement of human and animal populations, maximising opportunities for the vector and parasite. Although the direct relationship between biodiversity and disease transmission is uncertain, human settlement and behaviour clearly influence parallel changes in biodiversity and the epidemiology of zoonotic disease.

92

\section{Communicating Zoonoses: Exploring Risks, Perceptions, Practices and Beliefs Among Pastoralists in Northern and Eastern Tanzania}

\author{
Peter Mangesho ${ }^{1}$, Moses Ole Neselle, Esron D. Karimuribo, James E. Mlangwa, Janet George, Kevin Queenan, \\ Leonard E.G. Mboera, Richard Kock, Barbara Haesler and Mark Rweyemamu \\ ${ }^{1}$ Southern African Centre for Infectious Disease Surveillance
}

\begin{abstract}
Suspected zoonoses are reported through the medical authorities among pastoral communities in Tanzania. There is limited knowledge on how pastoral communities understand zoonoses in relation to their livelihoods, culture and their wider ecology. This study was carried out to assess knowledge and perceptions on zoonoses among pastoralists in Ngorongoro, Kibaha and Bagamoyo Districts. Qualitative interviews and participatory epidemiology were used. The understanding amongst pastoralists of zoonotic disease in these areas is still evolving, as there is no specific term in the local language that describes it. Pastoralists from Ngorongoro possessed a higher understanding on the existence of a number of zoonoses than others. Understanding of zoonoses could be categorized into two broad groups: a local syndromic framework, specific symptoms of a particular ailment in humans concurred with symptoms in animals, and the biomedical framework. While the knowledge about zoonoses is increasing, social and cultural practices maintaining social cohesion and certain passage rites will still put pastoralists at risk of zoonoses. Well-managed, equipped meat inspection practices could be used to advocate about zoonoses. These findings show how health trends are perceived, and how epidemiology and biomedicine are shaping pastoralists health perspectives while transforming people's conceptualization of life and health.
\end{abstract}




\title{
Risk Factors and Micro-geographical Heterogeneity of Schistosoma haematobium in Ndumo Area, uMkhanyakude District, KwaZulu-Natal, South Africa
}

\author{
Tawanda Manyangadze ${ }^{1}$, Moses John Chimbari $^{2}$, Michael Gebreslasie ${ }^{3}$ and Samson Mukaratirwa ${ }^{4}$ \\ ${ }^{1}$ School of Nursing and Public Health, Unversity of KwaZulu-Natal \\ ${ }^{2}$ College of Health Sciences, University of KwaZulu-Natal \\ ${ }^{3}$ School of Agriculture, Earth and Environmental Sciences, University of KwaZulu-Natal \\ ${ }^{4}$ School of Life Sciences, University of KwaZulu-Natal
}

This study determined the spatial distribution of Schistosoma haematobium and related risk factors in Ndumo area, uMkhanyakude District, KwaZulu-Natal province in South Africa. A sample of 435 schoolchildren between 10 to 15 years old from 10 primary schools was screened for S. haematobium using the filtration method. The GPS for their homesteads were recorded. Getis-Ord Gi ${ }^{\star}$ and Bernoulli model were used to determine the hotspots of S. haematobium. Semiparametric-Geographically Weighted Regression (s-GWR) model was used to predict the spatial distribution of S. haematobium in relation to environmental and socio-economic factors. Significant $S$. haematobium cases and infection intensity clusters $(\mathrm{p}<0.05)$ were observed. The s-GWR model performance was low $\left(\mathrm{R}^{2}=0.45\right)$ and its residuals did not show autocorrelation (Moran's $I=-0.001$; z-score $=0.003$ and $p$ value $=0.997$ ) indicating that the model was correctly spelled. The coefficients for distances of homesteads from operational piped water collection points, distance from open water sources, religion, toilet use, household head and places of bath and laundry varied at local scale and were significant ( $\mathrm{t}$-values \pm 1.96$)$ in some areas. This evidence may be used for control and management of the disease and further research is required to improve the performance of the s-GWR model.

\section{Building One Health Capacity for EID and Zoonoses Control and Prevention at Local Goverment Level: A Pilot Project in Three Districs in Indonesia}

\author{
Amy Mardiatmi ${ }^{1}$, Gos Ahmad Gozali ${ }^{2}$, Andro Andri Jatikusumah ${ }^{2}$, Azhar Muhammad Azhar ${ }^{2}$, Daisy Daisy \\ Joice $^{2}$, Elly Sawitri ${ }^{2}$, Endang Burni ${ }^{3}$, Irzal Irzal; Azhar ${ }^{4}$, Luuk Schoonman ${ }^{2}$, Yunus Mahmud ${ }^{2}$, Koko Ratmoko \\ Eko Saputro $^{2}$, Ratna Vitta Ekowati, Rince Morita Butar Butar, Ketut I Ketut Diarmita and James Mc. Grane \\ ${ }^{1}$ Directorate Animal Health, MoA \\ ${ }^{2} \mathrm{FAO}$ \\ ${ }^{3} \mathrm{MoH}$ \\ ${ }^{4}$ Ministry of Environment and Forestry
}

Whereas it is widely accepted that thecontrol and prevention of zoonoses and emerging infectious diseases (EID) in the world requires a One Health approach, One Health capacity building and implementation at local government (field) level is often challenging. We describe development of a training curriculum and practical approaches to One Health-focused zoonoses and EID control and prevention in Indonesia. The training targeted sub-district level staff from animal health centres (puskeswan), human health centres (puskesmas), and forestry field officers (polhut) in three districts in Indonesia, identified as potentially having a higher EID risk profile. Previous lessons learned from integrated disease detection, control and prevention activities between human and animal health officers on highly pathogenic avian influenza (HPAI) and rabies were included in curriculum development. This EID training also targeted district staff from the wildlife and forestry department to detect high mortality and unusual events in wildlife at an early stage. It was concluded that for early detection and control of zoonoses and EID an appropriate coordination, integration and information sharing mechanism between the Ministry of Agriculture, Ministry of Health and Ministry of Environment and Forestry is needed at district, provincial and central level. 


\title{
A Multidisciplinary Approach for the Surveillance of Possible Zoonotic Bat Pathogens in South Africa
}

\author{
Wanda Markotter ${ }^{1}$, Muriel Dietrich ${ }^{1}$, Marike Geldenhuys ${ }^{2}$, Petrus Janse van Vuren ${ }^{3}$, Teresa Kearney ${ }^{4}$, Stewart \\ McCulloch $^{1}$, Marinda Mortlock ${ }^{1}$, Ernest Seamark ${ }^{5}$, Jacqueline Weyer ${ }^{3}$ and Janusz Paweska ${ }^{3}$ \\ ${ }^{1}$ Centre for Viral Zoonoses, University of Pretoria \\ ${ }^{2}$ National Institute for Communicable Diseases \\ ${ }^{3}$ Ditsong National Museum of Natural History \\ ${ }^{4}$ AfricanBats
}

In addition to opportunistic short term surveillance, this study included longitudinal surveillance and the possibility of seasonal variation of virus prevalence in bat species in South Africa. In 2003 we initiated surveillance in bats with the initial objective to identify rabies-related lyssaviruses but this has since expanded to include several other pathogens. We used nucleic acid detection techniques to detect viral genomes and virus neutralization assays to test for presence of antibodies. We reported ten new isolations of the rabies related viruses, Lagos bat and Duvenhage. We indicated that frugivorous bats (Epomophorus wahlbergii and Rousettus aegyptiacus) maintain a high level (30-67\%) of seropositivity against Lagos bat virus and Nycteris thebaica and Miniopterus natalensis tested seropositive for Duvenhage virus. RT-PCR and metagenomics identified members of the Paramyxo, Filo, Adeno-, Herpes-, Picorna, Influenza and Coronaviridae. Longitudinal studies, specifically focused on cave dwelling fruit bats and measuring the presence of LBV antibodies over time, indicated cyclic fluctuation of antibodies with marked increases shortly after the parturition period, identifying high risk periods for spillover. We identified several viruses that are being shed into the environment, potentially infecting other species and the collective data can be used to inform prevention and control strategies.

1099

\section{Challenges and Opportunities in Developing One Health Approaches to Studying Zoonotic Diseases - Experiences in South Africa}

\author{
Wanda Markotter ${ }^{1}$ and Janusz Paweska ${ }^{2}$ \\ ${ }^{1}$ Centre for Viral Zoonoses, Department of Medical Virology, Faculty of Health Sciences, University of Pretoria \\ ${ }^{2}$ Centre for Emerging and Zoonotic Diseases, National Institute for Communicable Diseases of the National Health Laboratory \\ Services
}

Old and new zoonotic pathogens continue to emerge, many of which pose significant challenges to animal and human health communities. In the last few decades majority of emerging and remerging pathogens of epidemic prone potential originated from a zoonotic source. Development of sustained and effective prevention, detection and control strategies for these pathogens requires a One Health approach. This presentation will share the knowledge and results obtained after implementation of a One Health programme focusing on zoonotic viruses in bats in South Africa. Bats host a suite of viruses with severe public health consequences including SARS-like and MERS coronaviruses, Marburg, Ebola, Hendra, Nipah as well as rabies and rabies related lyssaviruses. Factors such as the availability of food resources, co-infecting parasites, age, reproductive status, migration and population density have all been demonstrated to increase or decrease the risk of viral infections within bat populations. Longitudinal surveillance on cave dwelling bats, measuring the presence of rabies related and filovirus antibodies over time, indicated cyclic fluctuation with marked increases shortly after the parturition period. We identified several viruses that are being shed into the environment, potentially infecting other species. This information together with our understanding of bat-human interactions is used to formulate feasible public health recommendations to prevent spill-over infections to humans and other animals. 


\section{Supporting Policy Implementation Through Collaboration: Sustainable Solutions to the Food and Nutrition Security Challenge in Tanzania}

Wende Maulaga $^{1}$, Elpidius Rukambile ${ }^{2}$, Robyn Alders ${ }^{3}, J^{\prime}$ lia de Bruyn ${ }^{4}$, Ezekiel Muyengi ${ }^{5}$, Elizabeth Lyimo ${ }^{6}$, Grace $\mathrm{Moshi}^{7}$, Brigitte Bagnol ${ }^{8}$, Furaha $\mathrm{Mramba}^{2}$ and Robyn McConchie ${ }^{9}$

${ }^{1}$ Tanzania Women Leaders in Agriculture and Environment

${ }^{2}$ Tanzania Veterinary Laboratory Agency

${ }^{3}$ Faculty of Veterinary Science \& and Charles Perkins Centre, University of Sydney, Sydney, Australia

${ }^{4}$ Faculty of Veterinary Science \& and Charles Perkins Centre, University of Sydney, Sydney, Australia

${ }^{5}$ Mikocheni Agricultural Research Institute Tanzania

${ }^{6}$ Tanzania Food and Nutrition Centre

${ }^{7}$ Ministry of Health and Social Welfare

${ }^{8}$ International Rural Poultry Centre, Kyeema Foundation, Maputo, Mozambique

${ }^{9}$ University of Sydney, Sydney, Australia;

Despite increases in agricultural production over the past two decades, undernutrition rates in children have not diminished significantly in many developing countries. In Tanzania stunting in children under five, a major determinant of individual development, was estimated to be $34 \%$ in 2014 . There has been an ongoing disconnect between agricultural policies and contemporary nutritional challenges, the persistent problem of micronutrient undernutrition and child stunting. Furthermore, the emerging challenges of other nutritional disorders coupled with food imbalances at household level and adverse environmental disturbances contribute to widening the gap in implementing proposed interventions. Our research is exploring how a multidisciplinary and multisectoral working team covering human and livestock health, food and nutrition security and related policy-making agencies, can create a cohesive team focused on delivering long term solutions. The composition of the Tanzanian Country Coordinating Committee (CCC) of the 'Strengthening food and nutrition security through family poultry and crop production in Tanzania and Zambia' has made acknowledged contributions to nutrition-sensitive policy interventions at District and National levels. Linkages between the CCC and participating households have enabled community members to raise issues across a broad range of nutrition-sensitive interventions.

1026

\section{Designing a One Health Surveillance Strategy for Emerging Diseases: Big Data, Dynamic Challenges, and Encouraging Step-wise Successes}

Jonna Mazet $^{1}$, Christine Johnson ${ }^{1}$, David Wolking ${ }^{1}$, William B. Karesh ${ }^{2}$, Peter Daszak ${ }^{2}$, Damien O. Joly ${ }^{3}$, Nathan Wolfe ${ }^{3}$, Sarah Olson ${ }^{4}$, Suzan Murray ${ }^{5}$, Kirsten Gilardi ${ }^{1}$, Woutrina A. Smith ${ }^{1}$, Simon J .Anthony ${ }^{6}$, Tracey Goldstein $^{1}$ and The PREDICT Consortium ${ }^{7}$

${ }^{1}$ University of California, Davis

${ }^{2}$ EcoHealth Alliance, New York

${ }^{3}$ Metabiota, San Francisco

${ }^{4}$ Wildlife Conservation Society, New York

${ }^{5}$ Smithsonian Institution, Washington

${ }^{6}$ Center for Infection and Immunity, Mailman School of Public Health, Columbia University, New York

${ }^{7}$ The PREDICT Consortium 
In an effort to move from the current reactive disease response paradigm to one of prevention and preparedness, the USAID's PREDICT Consortium has designed and implemented a targeted, risk-based strategy, based not on syndromic sentinels of disease but on detecting viruses early, at their source, where intervention strategies can be implemented before there is opportunity for spread. As a result, the team has advanced One Health capacity in more than 30 countries in emerging infectious disease hotspot regions. Environmental, host, and behavioral data are collected, and samples assayed for the presence of potential zoonoses. In addition to detecting approximately 200 known viruses, we have identified more than 800 previously undetected viruses. By combining these discoveries with data on human-wildlife contact and potential pathogenicity, we are assessing risk to inform mitigation strategies. Focusing our work where environments, human behaviors, and market systems are changing in ways that are conducive to the spillover of viruses among hosts, we locate areas posing the highest risk for exposure; detect and better characterize pathogens of epidemic and pandemic potential; identify significant animal reservoirs and amplification hosts of viruses; and provide information needed to efficiently design intervention strategies that target disease emergence, amplification, and spread.

794

\title{
Community of Practice Evaluation: A Case Study with CoPEH-Canada Exploring What Works
}

\author{
Kaileah McKellar ${ }^{1}$, Johanne Saint-Charles ${ }^{2}$, Whitney Berta ${ }^{1}$, Donald C. Cole ${ }^{1}$ and Rhonda Cockerill ${ }^{1}$ \\ ${ }^{1}$ University of Toronto \\ ${ }^{2}$ Université du Québec à Montréal
}

This paper presents results from a qualitative case study of the Canadian Community of Practice (CoP) in Ecosystems Approaches to Health (CoPEH-Canada). The case study aimed to enrich understanding of CoPEHs, specifically to examine processes and outcomes related to $\mathrm{CoP}$ resilience and sustainability from the perspective of early-career members. The secondary purpose was to test an Evaluation Framework for Extra-Organizational CoPs (developed by the authors) in guiding a case study and informing theory. This framework sets out levels of analysis (e.g. individual, collective) and types of value (e.g. learning, professional connections). This case contributes detailed information regarding the mechanisms through which CoP work (e.g., socialization, individual and collective leaning outcomes as drivers for continued participation). The study also provides a deepened, more nuanced understanding of the resilience and sustainability of CoPEHs, particularly with respect to participation of early-career members. The discussion outlines the extent to which the framework can be applied to different CoPEHs and other forms of partnership in the ecohealth and one health fields (e.g. collaborations between CoPEHs, One Health networks) and the adaptations which could be helpful for evaluations guiding the development of CoP resilience.

\section{3}

\section{Climate Change Vulnerability to Dengue at a Colombian Watershed}

Fabian Mendez ${ }^{1}$, Daniel Elias Cuartas ${ }^{1}$, Diana Maria Caicedo ${ }^{1}$, Delia Ortega ${ }^{1}$, Vikas Kumar ${ }^{2}$, William Ocampo ${ }^{3}$ and Marta Schuhmacher ${ }^{2}$

${ }^{1}$ Universidad del Valle

${ }^{2}$ Universitat Rovira I Virgili

${ }^{3}$ Universidad Javeriana Cali

Climate change will impact the peoplés health worldwide. Among other effects, climate change will increase extreme weather events that have the potential to impact the occurrence of vector borne diseases like dengue with a differential pattern of disease distribution according to local contexts. In this regard, vulnerability assessment is needed to analyze 
different aspects of complex ecological and social systems to intervene and reduce impacts on health. With this purpose, a fuzzy inference system was built including exposure, sensitivity, non-climatic factors and adaptive capacity in a comprehensive model. Data was obtained from primary and secondary sources from multiple sectors and vulnerability was estimated in 42 municipalities in the Cauca river geographical valley at South Western Colombia finding mostly high (69\%) and medium $(31 \%)$ levels of vulnerability. In particular this result is consequence of high exposure to extreme weather events (87\%), medium level of sensitivity (59\%), and average levels of adaptation capacity (89\%) and non-climatic factors (78\%). The processing system allowed to handle the imprecision and uncertainty associated with climate change in the case of dengue. However, given that vulnerability is changing over time, constant evaluation is required for building sound adaptation strategies that take into account local and global influences and consider conditions of local development.

\section{5}

\section{Leprosy in Red Squirrels in the British Isles; A Newly Identified Wildlife Reservoir}

Anna Meredith ${ }^{1}$, Charlotte Avanzi ${ }^{2}$, Jorge Del Pozo ${ }^{1}$, Andrej Benjak ${ }^{2}$, Karen Stevenson ${ }^{3}$, Joyce McLuckie ${ }^{3}$, Darren Shaw ${ }^{1}$, Stewart Cole ${ }^{2}$ and Vic Simpson ${ }^{4}$

${ }^{1}$ Royal (Dick) School of Veterinary Studies and The Roslin Institute, University of Edinburgh

${ }^{2}$ Global Health Institute, Ecole Polytechnique Federale de Lausanne

${ }^{3}$ Moredun Research Institute

${ }^{4}$ Wildlife Veterinary Investigation Centre, Chacewater, Cornwall

Leprosy, once an endemic human disease in the British Isles, arises from infection with Mycobacterium leprae or the recently discovered Mycobacterium lepromatosis, and remains a public health problem in certain low and middle-income countries with $\sim 220,000$ new cases reported annually. In 2014 we reported a novel mycobacterial dermatitis of red squirrels (Sciurus vulgaris) in Scotland, associated with infection with Mycobacterium lepromatosis, and histologically comparable with human diffuse lepromatous leprosy. Since then there have been increasing British reports of red squirrels presenting with leprosy, which we have investigated using genomics, histology and serology. We have now detected and genotyped leprosy bacilli in both overtly diseased and seemingly healthy squirrels from Scotland, England, and Ireland, including a large cluster from Brownsea Island, England where historic evidence indicates observation of affected animals for over forty years. Our findings show that red squirrels are a potential zoonotic source of leprosy and demonstrate that a pathogen can remain undetected in the environment centuries after its clearance from the human reservoir. Further surveys of animal reservoirs of leprosy bacilli are warranted since they may contribute to the inexplicably stubborn plateau in the incidence of the human leprosy epidemic despite effective and widespread treatment with multidrug therapy.

999

\section{EPIDEMIA: An Online Platform for Data Acquisition, Integration, and Analysis to Support Ecological Forecasting of Malaria Outbreaks}

Christopher Merkord ${ }^{1}$, Yi Liu ${ }^{1}$, Michael DeVos ${ }^{1}$, Adam Dosch $^{1}$, Abere Mihretie ${ }^{2}$, Alemayehu M. Lemma ${ }^{2}$, Teklehaymanot Gebrehiwot ${ }^{3}$, Mastewal Worku Lake ${ }^{3}$, Estifanos Bayabil ${ }^{2}$ and Michael Wimberly ${ }^{1}$

${ }^{1}$ South Dakota State University

${ }^{2} H D A M A$

${ }^{3} A R H B$ 
Malaria early detection and early warning systems are important tools for public health decision makers, especially in regions where malaria transmission is unstable and dependent on environmental conditions such as rainfall and temperature. We designed and implemented the EPIDEMIA online platform, an automated tool for forecasting malaria outbreaks in the Amhara Region of Ethiopia. The components of the system handle epidemiological data acquisition whereby public health collaborators upload malaria surveillance data, environmental data acquisition whereby our previously-developed EASTWeb software continuously downloads and summarizes environmental monitoring data derived from satellite remote-sensing products, data integration which harmonizes the various datasets, modeling and forecasting of outbreaks using state-space models, and reporting via PDF, HTML, and Shiny app visualizations. Our modeling and forecasting subsystem uses a new data-driven dynamic linear model based on the Kalman filter with time-varying coefficients that are used to identify malaria outbreaks as they occur (early detection) and predict the location and timing of future outbreaks (early warning). The ecohealth informatics tools implemented in EPIDEMIA can be modified to incorporate new modeling techniques, applied to other geographic areas, or extended to other diseases linked to environmental conditions.

854

\title{
A Network Approach to the Study of Participation, Transdisciplinarity and Sustainability in Ecohealth Research
}

\author{
Frédéric Mertens ${ }^{1}$, Renata Távora ${ }^{1}$ and Johanne Saint-Charles ${ }^{2}$ \\ ${ }^{1}$ Centro de Desenvolvimento Sustentável-Universidade de Brasília \\ ${ }^{2}$ Université du Québec à Montréal
}

In ecohealth research, participatory and transdisciplinary approaches have been proposed in order to allow researchers, communities and policy makers to share and collaborate on complex ecosystem-related health issues, according to their perspectives, concerns and priorities. Social relations, such as information exchanges, collaboration, resource sharing and trust, are central to these collective processes of knowledge generation. We present a synthesis on the use of social network analysis (i) to map the relations among actors involved in participatory and transdisciplinary ecohealth research, and (ii) to characterize the structural characteristics of networks that emerge from these multi-stakeholders collaboration, and (iii) to understand how patterns of relation affect the capacity of the research process to promote health improvements and environmental sustainability. We also propose indicators, based on individual-level and whole network measures, to characterize several key aspects of ecohealth research, such as gender issues, equity in participation, sustainability of the research results, levels of pluridisciplinarity, adaptive capacity to social and environmental changes, robustness and resilience of the knowledge generation processes. Indicators are illustrated and discussed using several ecohealth case studies, including community development projects, health promotion initiatives and international governance programs.

\section{4}

\section{Food Safety Challenges and International Trade}

\section{Charles Milne \\ Department of Economic Development, Jobs, Transport and Resources, Biosecurity Branch}

Animal pathogens can have a direct impact on public health through the contamination of food. Salmonella, Campylobacter and $\mathrm{E}$ coli are recognised as being associated with foodborne infections worldwide and food is a recognised vehicle for the transfer of many other zoonotic organisms. Additionally, public perception of risk can result in food scares and reduced consumption in non zoonotic diseases such as Foot and Mouth disease. In order to trade internationally in animals and animal products reassurances are often required by importing countries regarding the freedom from contamination with zoonotic organisms with the potential to impact on human health. Robust surveillance systems, appropriate diagnostic tools, 
risk assessment and reliable certification arrangements are required to identify and manage risks associated with these infections and to provide the reassurances necessary to provide confidence to underpin both domestic and international trade.

\title{
521
}

\section{Integrative Approaches to Environment, Community \& Health: Innovations and Connections Across Local, Indigenous and Geospatial Knowledge}

\author{
Helen Moewaka Barnes \\ Massey University
}

Te Tai Ao is a research platform within Nga Pae o Te Maramatanga, Maori Centre of Research Excellence in Aotearoa New Zealand. Working in the domain of environmental integrity and sustainability we are building on a unique body of knowledge and practice, bringing together a multi-disciplinary team of Māori researchers and communities with expertise in developing solutions derived from Indigenous knowledge and science. The initial stages of developing a vision that can guide us locally, nationally and internationally will be discussed along with some key success factors and successes in working with indigenous groups in restorative action.

1024

\section{Disease Control: Benefits to Health, Development and Livelihoods}

\section{Subhash Morzaria \\ Food and Agriculture Organization of the UN}

The demand for livestock food products has lead to annual livestock production net growth globally since 1961, and it is expected to further grow to meet demands projected for 2030-2050. Concerns over a growing global demand for meat have emphasized livestock agriculture's role as a leading contributor to greenhouse gas emissions, as well as the anticipated land conversion to support both cattle raising and cattle feed. But could future demand actually be met with current production levels? Disease remains a major contributor to reduced efficiency in livestock production systems, with an estimated onefifth of production loss. Livestock management solutions for improving disease prevention and control will be discussed in the context of sustainable development.

\section{The Cull of the Wild: Can Wild Plant Foods Contribute to Healthy and Diverse Diets and Food Systems?}

Daniela Moura De Oliveira Beltrame ${ }^{1}$, Ayfer $\operatorname{Tan}^{1}$, Camila Neves Soares Oliveira ${ }^{2}$, Lidio Coradin ${ }^{1}$ and Danny Hunter $^{3}$

${ }^{1}$ Biodiversity for Food and Nutrition Project

${ }^{2}$ Ministry of the Environment Brazil

${ }^{3}$ Biodiversity for Food and Nutrition Project, Bioversity International

Despite poor attention to wild foods in food and nutrition policies and strategies they do play an important role in people's diets and livelihood strategies. One reason for this neglect by policy and decision makers is believed to be the limited knowledge on the value and contribution of wild foods to food security and nutrition. There are also many other barriers preventing the wider use of wild foods for food security. The Biodiversity for Food and Nutrition (BFN) Project, a multicountry project, has been working to address these challenges by improving the enabling environment for wild plant foods 
by: improving the evidence base for wild plants by undertaking composition analysis of about 150 prioritized species, based on their nutritional and socioeconomic potential; strategically targeting public policies and markets with the greatest potential for mainstreaming these species e.g. by diversifying public food procurement and school feeding; and, improving national capacity, partnerships and awareness through alliances with universities, governments, civil society, nutritionists and dieticians, chefs and national food movements. This presentation will demonstrate the benefits of a diversified diet that includes wild, nutritious species as well as how these species can be a source of work and income for family farmers.

468

\title{
Proximity of Private Water Wells to Water Public Health Test Facilities in Alberta
}

\author{
Abraham Munene and David Hall \\ University of Calgary
}

More than 400,000 rural Albertans use well water for domestic purposes. In contrast to municipal water supply systems which undergo routine testing and treatment, the responsibility of rural well water testing is left to private owners. Fewer than $10 \%$ of well owners in Alberta routinely test their well water. Distance to water testing facilities may be an important factor limiting well water sample submissions. This study describes the proximity of water wells to public health test facilities in the province. Using service area analysis, we determined the proportion of domestic wells within five impedances $(15,30,45,60$, and $>60 \mathrm{~min})$ of test facilities. There were 19,102 water wells drilled for domestic use within Alberta during the period examined (2006-2015). One hundred and nine water public health test facilities were identified within the province. $7843(41 \%), 7666(40.1 \%), 2069$ (10.8\%), 437 (2\%), and $1087(6 \%)$ water wells were located within one-way travel times of $15,30,45,60$, and $>60$ min respectively of a water public health test facility. Based on one-way travel time, we concluded distance may be a barrier to voluntary water sample submission for testing.

1029

\section{Achieving EcoHealth Principles through Community Engagement}

\author{
Rosemary Musesengwa ${ }^{1,2}$ and Moses Chimbari ${ }^{1,2}$ \\ ${ }^{1}$ University of KwaZulu-Natal, Durban, South Africa \\ ${ }^{2}$ International Association for Ecology and Health
}

Community Engagement (CE) in health research ensures fulfilment of the EcoHealth principle of stakeholder involvement. The challenge for researchers is to attain the necessary skills and resources to implement CE. We describe CE in an EcoHealth community based research project in two rural, vulnerable, research naive communities in Southern Africa. A qualitative, multiple case study approach was used. Data was collected through Participatory Rural Appraisals, FGDs, KII, and observations. The engagement process included: (i) Extensive formative research activities such as PRAs (ii) Introductions to the political, traditional and administrative leaders, (iii) Establishing a community advisory mechanism through Community Advisory Boards, (iv) Community empowerment through education of ordinary community members at biannual meetings, training of community research assistants (CRA) and utilizing indigenous knowledge citizen science groups. Empowerment of CRAs and citizen science groups enabled the study to initiate sustainable community initiated research activities. High stakeholder and community involvement also ensured high research uptake even at policy level. It is possible to achieve the EcoHealth pillars through enhanced, deliberate CE. EcoHealth implementation should be consistent with community values and attitudes. Achieving EcoHealth and CE is constrained by community research literacy levels, time, human and financial resources but creates a conducive research environment. 


\title{
Prevalence of Fasciolosis in Cattle, Sheep and Goats Slaughtered in Slaughter Slabs and Knowledge of Livestock Handlers in Trans NZOIA West Sub county, Kenya
}

\section{Protus Musotsi}

Moi university

A 5 year secondary data retrospective study was carried out to determine the prevalence of fasciolosis among ruminants slaughtered in Trans-Nzoia West, Kenya. Relationship between prevalence and rainfall patterns was determined and the knowledge of meat sellers and farmers in relation to fasciolosis assessed.

A total of 104,221 cattle, sheep and goats were slaughtered in the 5 year period in which 6,098 (5.85\%) were infested with fasciolosis prevalence of $6.52,6.08$ and $4.10 \%$ in cattle, sheep and goats respectively.

Most meat sellers $(72.2 \%)$ were able to identify fasciolosis in infested livers, $88.9 \%$ reported liver flukes infestation resulted in loss of income while 58.3\% were not aware of nutrition depletion associated with fasciolosis. Of the farmers interviewed, 52.9\% grazed in swampy areas, 66.7\% dewormed after 3 months, $78.4 \%$ had heard about fasciolosis and $80.0 \%$ did not know the cause of fasciolosis. Majority (95.0\%) were not aware of the snail species spreading liver flukes while $77.5 \%$ did not know that fasciolosis was a zoonotic disease.

Fasciolosis was prevalent in the area. There was no significant relationship between rainfall and fasciolosis prevalence. Most farmers were not aware of its cause and zoonotic nature. Fasciolosis control should be approached at multidisciplinary angle.

1058

\section{Feeding Vegetable Wastes to Backyard Pigs in Benguet Province, Philippines: Boon or Bane?}

\author{
Monaliz Nagrampa ${ }^{1}$ and Van Joe Ibay ${ }^{2,3,4}$ \\ ${ }^{1}$ Benguet State University College of Veterinary Medicine \\ ${ }^{2}$ Animal Welfare Coalition, Manila \\ ${ }^{3}$ The University of Makati College of Allied Health Studies \\ ${ }^{4}$ Public Health Association of Australia
}

Farmers in developing countries like the Philippines have been relying on pesticide use to meet quantity and quality demands for food. Previous studies in Benguet, the largest vegetable producing province in the Philippines, showed farmers' dependence as soil, water and vegetable samples contain pesticide residues. Agricultural crops serve as major revenue source, likewise, tons of wastes from vegetable rejects and trimmings contributing an estimate of 60 tons daily in 2011. The local government treat wastes as a problem, while some natives view them valuable by utilizing them as feed to backyard pigs. Pork products sourced from this type of practice creates public health risks from exposures to carcinogenic chemicals, chronic disease and antimicrobial resistance. The objective of this study is to provide an assessment of farmers' knowledge, attitudes and practices on pesticide use and residue build up (in meat). Mixed methods will be utilized in performing the study, consisting of constructive grounded theory and quantitative methods. The results will serve as basis creating evidenced base policies for regulatory and surveillance purposes of drug residue in food and agricultural products. 


\section{Building One Health University Education Systems: A Sustainable Approach to One Health Workforce Development By the Ohcea Network}

Irene Naigaga ${ }^{1}$, Timothy Wakabi ${ }^{1}$, Agnes Yawe ${ }^{1}$, Winnie Bikaako ${ }^{1}$, Milly Nattimba ${ }^{1}$, Hellen Amuguni ${ }^{2}$, John David Kabasa $^{1}$ and William Bazeyo ${ }^{1}$

${ }^{1}$ One Health Central and Eastern Africa

${ }^{2}$ Tufts University, Cummings School of Veterinary Medicine

The One Health approach is a strategy to promote multi-sectoral collaboration across disciplines to advance human health, animal health, and ecosystem health. This synergism is needed to achieve more rapid, mutually beneficial and effective responses to potential or existing risks that originate at the animal-human-ecosystems interface. Driven by the primary target and passion to have communities healthier, wealthier and safe, the One Health Central and Eastern Africa (OHCEA) network has embarked on developing One Health Higher Education Systems in her 17-member university institutions. The objective is to produce a One Health graduate having transformative knowledge, ability, attitude and networking skills to prevent and mitigate risks that originate at the animal-human-ecosystems interface. To produce this graduate, universities are building four pillars, namely: A One Health university policy framework/strategy and implementation plan; One Health curricula through massive curriculum reviews to incorporate One Health competencies; One Health curriculum delivery mechanism; and One Health trainers trainers capacitated in terms of One Health skills and facilities. This paper will provide a framework for building One Health curricula pillar and propose strategies on how the revised curricula can be delivered from a One Health perspective.

936

\section{When Pigs Fly: The Role of the International Live Swine Trade in the Emergence of the 2009 Pandemic Influenza A Virus}

Martha Nelson, Ignacio Mena, Adolfo Garcia-Sastre, Philippe Lemey, Cecile Viboud

National Institutes of Health

In early 2009, a novel reassortant influenza A/H1N1 virus of swine origin caused a major outbreak in Mexico and quickly spread globally, resulting in the first influenza pandemic of the 21st century. Segments of the pandemic virus (pdmH1N1) are related to an avian-like Eurasian swine virus lineage (EAsw) that has never been identified in the Americas. It is therefore unclear how a virus that most likely evolved in Asian swine caused its first outbreak in humans in Mexico. In this talk I will describe how increased sequencing of influenza A viruses in swine globally since 2009 has advanced our understanding of the global ecology of the virus, including the key role of international trade of live swine in its intercontinental spread. I will introduce new data from Mexican swine that resolves the debate over whether the swine virus that gave rise to pdmH1N1 emerged in Asia or the Americas. Recognizing how long-distance viral migration driven by the animal trade has altered the global landscape of influenza virus diversity in swine in recent decades is key to identifying new geographical hotspots for the emergence of new pandemic viruses of swine origin.

478

\section{Advancing Ecohealth in Southeast Asia and China: Findings and Lessons from the Field Building Leadership Initiative}

Hung Nguyen-Viet ${ }^{1}$, Wiku Adisasmito ${ }^{2}$, Pattamaporn Kittayapong ${ }^{3}$, Fang Jing ${ }^{4}$, Xuan Tung Dinh ${ }^{5}$ and Phuc Pham Duc ${ }^{6}$

${ }^{1}$ International Livestock Research Institute (ILRI) 


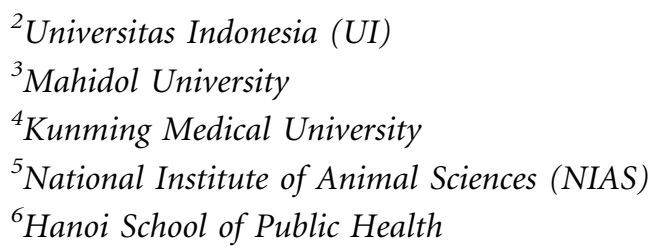

The Field Building Leadership Initiative (FBLI), supported by the International Development Research Centre (IDRC), has been working to understand and address intensive agricultural practices and associated health risks in Southeast Asia and China. Developed jointly by research centres in China, Indonesia, Thailand and Vietnam, and launched in 2012, this fiveyear initiative allows researchers and their partners to carry out research, capacity building, and knowledge translation to inform practice and policy. After 5 years of implementation, we report here the results of four research case studies conducted in 4 countries using an transdisciplinary approach involving stakeholders from the design phase to implementing interventions. Researchers and partners undertook research on a number of issues i) Pesticide use and its impact on human health and agricultural ecosystems in China; ii) Human and animal waste management in Vietnam; iii) Rubber plantations and vector-borne diseases in Thailand; and iv) Small-scale dairying in Indonesia. For capacity, a critical mass of teaching materials have been developed, students and ecohealth practitioner trained, in particular ecohealth future leaders trained widely in the region. Finally, we present how knowledge translation has been implemented to bring research evidences into policy and actions to improve health and environment.

\section{Healthy Family, Healthy Forest: Practical Solutions for Long-term Conservation Outcomes in Papua New Guinea}

\section{Mikal Nolan}

Tree Kangaroo Conservation Program-PNG

A Category VI IUCN Protected Area, the YUS Conservation Area is the only protected area of its type in Papua New Guinea - landscape scale, wholly owned by local people, with the support of the PNG Government for long-term protection. YUS is home to a number of human settlements as well as rare and endemic flora and fauna. The Tree Kangaroo Conservation Program-PNG strives to harmonize the co-existence of human activity and habitat protection for endemic and endangered wildlife within the protected area. This is especially important given the remoteness of the communities and their dependency on natural resources.

TKCP-PNG's Health Family, Healthy Forest project offers a practical solution that seeks to relieve local pressures on the forest ecosystems without degrading the biodiversity of the protected area. The project utilizes the integrated PopulationHealth-Environment (PHE) approach. The key to the success of the project is providing the training to the appropriate members of the community in order to carry out adult and youth peer-education. TKCP-PNG incorporates the PHE approach into livelihood activities, land-use planning, and youth programs. This proposal would explore the project as an example of an integrated and practical solution for promoting both a healthy environment and a healthy population.

\section{2}

\section{Ecological Niche Modeling of Rift Valley Fever Virus Vectors In Baringo Kenya}

Alfred Ochieng ${ }^{1}$, Mark Nanyingi ${ }^{2}$, Edwin Kipruto ${ }^{1}$, Isabella Ondiba ${ }^{3}$, Fred Amimo ${ }^{1}$, Chris Oludhe $^{3}$, Dan Olago ${ }^{3}$, Isaac Nyamongo ${ }^{1}$ and Benson Estambale ${ }^{1}$

${ }^{1}$ Jaramogi Oginga Odinga University of Science and Technology 
${ }^{2}$ Department of Biomedical Sciences, Colorado State University, Fort Collins, CO, USA. Department of Public Health, Pharmacology and Toxicology, University of Nairobi, Kenya

${ }^{3}$ University of Nairobi

Rift Valley fever (RVF) is a vector-borne zoonotic disease that has an impact on human health and animal productivity. The episodic RVF outbreaks are closely linked to interannual climate variability.This study evaluated the effect of climate change on RVF vector distribution in Baringo County, Kenya with an aim of developing risk maps for spatial prediction of RVF outbreaks. The study used vector presence data and Maximum Entryopy (MaxEnt) ecological niche modelling algorithm to predict the effect of climatic change on habitat suitability and the spatial distribution of RVF vectors. Data on species occurrence was obtained from longitudinal sampling of adult mosquitoes and larvae. We used current (2000) and future (2050) Bioclim climate data to model vector distribution. Model results predicted potential suitable areas with high success rates for Culex quinquefasciatus, Culex unvitattus, Mansonia africana and Mansonia uniformis. Under the present climatic conditions, the lowlands were found to be highly suitable for all vector species. Models based on future climatic conditions indicate an increase in the vectors' spatial range, exposing new human and animal populations to RVF risk. Soil types, precipitation in the driest quarter, precipitation seasonality and isothermality showed the highest predictive power for the four species.

\section{4}

\section{Human Health Consequences of Artisanal and Small-scale Gold mining in Ghana}

Eric Odei ${ }^{1}$, Niladri Basu ${ }^{2}$, Edith Clarke ${ }^{3}$, Allyson Green ${ }^{4}$, Benedict Calys-Tagoe ${ }^{5}$, Laurie Chan ${ }^{6}$, Mawuli Dzodzomenyo $^{5}$, Julius Fobil ${ }^{5}$, Rachel Long ${ }^{7}$, Richard Neitzel ${ }^{7}$, Samuel Obiri ${ }^{8}$, Lauretta Ovadje ${ }^{7}$, Reginald Quansah $^{5}$, Mozghon Rajaee ${ }^{7}$ and Mark L. Wilson ${ }^{5}$

${ }^{1}$ Korle Bu Teaching Hospital

${ }^{2}$ McGill University

${ }^{3}$ Ghana Health Service

${ }^{4}$ University of Michigan School of Public Health

${ }^{5}$ University of Ghana School of Public Health

${ }^{6}$ University of Ottawa

${ }^{7}$ University of Michigan School of Public Health

${ }^{8}$ Council for Scientific and Industrial Research-Water Resources Institute

This is one of three synthesis documents emanating from an Integrated Assessment (IA) that aims to increase understanding of artisanal and small-scale gold mining (ASGM) in Ghana. Given the complexities surrounding ASGM, an IA framework was utilized to analyze economic, social, health, and environmental data, and co-develop evidence-based responses with pertinent stakeholders. This current analysis focuses on the health of ASGM miners and community members, and synthesizes extant data from the literature as well as co-authors' recent findings regarding the causes, status, trends, and consequences of ASGM in Ghana. The results provide evidence from across multiple Ghanaian ASGM sites that document relatively high exposures to mercury and other heavy metals, occupational injuries and noise exposure. The work also reviews limited data on psychosocial health, nutrition, cardiovascular and respiratory health, sexual health, and water and sanitation. Taken together, the findings provide a thorough overview of human health issues in Ghanaian ASGM communities. Though more research is needed to further elucidate the relationships between ASGM and health outcomes, the existing research on plausible health consequences of ASGM should guide policies and actions to better address the unique challenges of ASGM in Ghana and potentially elsewhere. 


\title{
Landscapes and Livelihoods to Unpack the Complex Socio-Ecological Dynamics of Human Health: A Case Study from the Brazilian Amazon
}

\author{
Jordan Sky Oestreicher
}

There is a rich reservoir of knowledge on landscapes and livelihoods - both as research concepts and as frameworks for action - that has not yet been widely applied to human health; as such they represent an important contribution to the ecohealth toolkit. Landscapes and livelihoods are outcomes of complex human-ecosystem interactions. In the Brazilian Amazon, the landscape is a mosaic of rainforest, pasture, crops and growing urban areas. The diverse ways people make a living, known as livelihoods, often involves fishing, hunting, farming, and ranching. With on-going development in the region, however, livelihood options and the landscape are rapidly changing. In turn, this is altering the movement of toxins in the environment or the cycling of pathogens and disease vectors. Here, we demonstrate how landscapes and livelihoods can be used to integrate multiple health concerns into a common analysis that draws on interdisciplinarity and complexity thinking. We exemplify using two public health concerns in the Brazilian Amazon: one that is eco-toxicological (methylmercury exposure) and one that is vector-born (Chagas disease risk). Through an analysis of landscape patterns and livelihood activities, we highlight the complex, interrelated, and cross-scale social and ecological processes that drive the emergence of these two health concerns. We also identify pathways for public health interventions and ecosystem management.

\section{9}

\section{Operationalising Transdisciplinarity: How Epidemiologists, Anthropologists and Economists Contributed To Taenia Solium Control in Northern Lao PDR}

\begin{abstract}
Anna Okello, Phouth Inthavong, Boualam Khamlome, Amanda Ash, Walter Okello, Kevin Bardosh, Tassilo Tiemann and John Allen

Suspicions regarding the existence of hyperendemic Taenia solium foci in Lao PDR were confirmed in 2013, when a crude taeniasis prevalence of $30.6 \%$ (95\% C.I. 25.5 - 38.9\%) was identified in a remote village of 300 inhabitants in the north. By early 2015 , a control intervention in both the human and pigs had achieved a 78.7\% reduction of adult parasite levels to $6.5 \%$ (95\% C.I. 3.4-9.5\%). Whilst epidemiological studies were key to demonstrating the overall goal of parasite reduction, careful consideration of diagnostic, anthropological and socioeconomic factors was necessary to balance community acceptability with scientific rigour and economic feasibility. Anthropological assessments flagged previously unknown transmission drivers - such as the significance of raw pork consumption for sacrificial ceremonies - highlighting the need to consider social realities in the design of community-level interventions. Similarly, the requirement to optimise cost effectiveness for policy purposes was a key motive for building soil transmitted helminths and classical swine fever control into the design from the outset. Garnering advocacy and action for disease control requires foresight into not just how diseases are transmitted, but how communities function and how policies are developed and funded. This pilot intervention showcases how transdisciplinarity contributes to the design, monitoring, evaluation - and ultimately the success - of ground level interventions.
\end{abstract}

574

\section{Integrating Market Chain Assessments with Porcine Zoonoses Risk Analysis in Two Cross-Border Value Chains in Lao PDR}

Anna Okello, Tassilo Tiemann, Phouth Inthavong, Boualam Khamlome, Ammaly Phengvilaysouk, Soukhan Keonouchan and John Allen Australian Animal Health Laboratory 
Integration of market chain and zoonoses risk analysis methodologies has the potential to improve our understanding of risk mitigation for transmission of human and animal diseases, including zoonoses. In this study, information gained from smallholder pig farmer/trader interviews was integrated with serological surveys for pig-associated zoonoses such as hepatitis E virus, Taenia solium and Trichinella to identify potential linkages between disease risk and pig production/slaughter in low input systems across the country. Trichinella and HEV exposure was high in both humans and pigs in both study areas, significantly associated with pig slaughter and the subsequent consumption and handling of raw pork products. $T$. solium demonstrated a strong geographical and ethnic association with the northern study area bordering Vietnam. With the right knowledge and accessible, affordable inputs, the majority of smallholder farmers indicated a willingness to increase economic investment in pig production, which could simultaneously improve livelihoods and decrease disease exposure through increased access to formal markets and improved slaughter processes. Assessing risk in this way highlights the importance of a systems-based approach to improving the understanding of the Lao PDR livestock sector in accordance with the country's recent accession to the World Trade Organization.

183

\title{
A Social Ecological Model of Farmers' Attitudes and Behaviours Towards the Control of Animal Diseases
}

\author{
Haini Pao, Dirk Pfeiffer, Elizabeth Jackson \\ Royal Veterinary College, University of London
}

The aim of the research is to understand broiler farmers' opinions, attitudes, and behaviours concerning on-farm biosecurity programmes in Taiwan. This mixed-method research involves two phases aimed at identifying social and epidemiological factors influencing the uptake of recommended on-farm disease management measures. Content analysis of face-to-face interviews with 25 farmers revealed the influence of macro socio-economic conditions on famers' attitudes. A social ecological model was then developed to explain the complexity of factors affecting on-farm biosecurity practices with multilevel social structure. Survey data were collected from 303 farmers to understand the current situation of production processes. Using categorical principal components analysis and two-stage cluster analysis, two types of clusters were identified: attitude clusters (based on 15 variables) and behaviour clusters (characterised by 30 variables), and these findings suggested it is essential to apply appropriate and specified behavioural change theories to groups of farmers. The novelty of this research lies in its wider relevance to Taiwan's poultry production industry in that it identifies the shortcomings of focussing primarily on epidemiological risk factors and not including social factors, as tends to be the case in most animal disease control activities.

869

\section{Integrative Approaches to Environment, Community \& Health: Learning from Local, Indigenous and Geospatial Knowledge in Northern BC, Canada}

\section{Margot Parkes \\ University of Northern British Columbia}

Integrative approaches to environment, community and health issues are demanding new tools and processes. Within our expanding digital toolscape is a growing suite of integrative approaches and geospatial tools that are being used to value and profile diverse knowledges in new ways, and create conversations across local, Indigenous, scientific and organisational knowledge, among others. This presentation will present findings from two interrelated projects underway in northern British Columbia, Canada that are developing, refining and applying integrative and geospatial tools to better understand and respond to the health implications of resource extraction and development in northern British Columbia: the "Nechako Watershed Portal", and the "Cumulative Impacts Research Consortium". Discussion will focus on how these 
complementary approaches are seeking to value local and indigenous knowledge, to foster diverse stakeholder engagement, and to inform intersectoral decision-making in relation to the cumulative environmental, community and health impacts of resource development. Attention will also be given to how efforts in BC compare to related efforts that are developing in other jurisdictions, including Canada, the US (Alaska and Hawaii), and Aotearoa/New Zealand.

1081

\section{Climate Change and Effects on Human and Animal Diseases}

\section{Jonathan Patz}

University of Wisconsin, Madison

Climate change poses large risks due to multiple exposure pathways though which increases in climatic extremes can threaten human and animal health. Case studies will be presented on both anthroponoses and zoonoses, including recent findings on El Niño and Zika virus in South America. Public health preparedness for the range of climate-sensitive diseases will be outlined, as well priority interventions that offer health "co-benefits" from greenhouse gas mitigation options. Examples of the latter include: air quality benefits from use of clean energy technology, opportunities to enhance physical fitness through "active" transportation (via walking and biking), and sustainable food systems via alternative protein sources and cropping methods. Climate change represents one of our greatest health threats, but at the same time, policies to confront fossil fuel combustion at the root of the global climate crisis, offer some of the greatest health opportunities of our times.

\section{3}

\section{Pandemic Predictions: Can Influenza Pandemics Ever be Anticipated?}

\section{Malik Peiris}

School of Public Health, University of Hong Kong

Influenza pandemics emerge from animal influenza viruses. The example of the 2009 pandemic highlighted the rapidity with which a newly emerged pandemic virus spreads globally, making it unlikely that conventional vaccines will be ready in time to mitigate the first wave of the pandemic. There are efforts to enhance surveillance and risk assessment of influenza viruses at the animal-human interface so as to identify viruses of highest potential risk, so that pre-emptive steps to initiate development of candidate vaccine strains can be implemented allowing a head-start in the vaccine development process. Such risk assessments may also help identify animal viruses of the greatest concern, so that interventions may be instituted to minimize risk of pandemic emergence. Recently, influenza virus risk assessment strategies have been developed to make this process more systematized and transparent. These include the Influenza Risk Assessment Tool (IRAT) developed by the US CDC, the Tool for Influenza Pandemic Risk Assessment (TIPRA) being developed by WHO with "One-Health" partners and FluRisk being developed with the European Food Safety Authority. Application of such approaches to risk assessing animal influenza viruses provides an understanding of knowledge gaps and will potentially lead to enhancing the process in an iterative manner.

\section{An Evidence-Based Approach to Improving Cost-effectiveness of Rabies Post Exposure Prophylaxis In High Risk Areas of Bhutan}

Kinley Penjor ${ }^{1}$, Kinley Penjor_V ${ }^{1}$, Chendu Dorji ${ }^{1}$, Chencho Dorjee ${ }^{2}$, Sithar Dorjee ${ }^{3}$, Joanna McKenzie ${ }^{3}$, Peter Jolly ${ }^{3}$ and Roger Morris ${ }^{4}$

${ }^{1}$ One Health Epidemiology Postgraduate Fellow, Massey University and Faculty of Nursing and Public Health, Khesar Gyalpo 
Uinversity of Medical Sciences of Bhutan

${ }^{2}$ Faculty of Nursing and Public Health, Khesar Gyalpo University of Medical Sciences of Bhutan

${ }^{3}$ Institute of Veterinary Animal and Biomedical Sciences, Massey University, New Zealand

${ }^{4}$ Morvet Ltd, Consultancy services in health risk management and food safety policy and programs, Masterton, New Zealand

Post exposure prophylaxis (PEP) is essential for rabies control, but expensive-comprising 6\% of Bhutan's medicines expenditure. Only a few of these people would have been exposed by a rabid dog. Rabies incidence in people in the high risk area adjoining India was 3.14/100,000, whereas without PEP it was predicted to be 19.24/100,000. PEP administration practices by clinicians in high rabies-risk areas were investigated to target PEP more precisely to people with a genuine risk of rabies exposure, avoiding excessive precautionary use. Health workers used a structured questionnaire whilst observing clinician-patient interviews to assess 276 suspected rabies exposure cases by 52 clinicians within 13 district and subdistrict hospitals. This provided evidence of the risk of rabies exposure associated with different forms of dog exposure, leading to development of a classification system which can be used to guide decisions on which patients should receive PEP. The national PEP guidelines will now be revised and training materials developed for clinicians. Savings from reducing PEP expenditure can potentially be diverted to reducing exposure of people through enhanced rabies control effort in dogs in the high-risk areas, further reducing the need for PEP by adopting an integrated One Health control policy.

\title{
142
}

\section{Participatory Learning and Action for Rabies Control in Bali}

\author{
Sang Purnama ${ }^{1}$, Arya Utami ${ }^{2}$ and Made Subrata ${ }^{1}$ \\ ${ }^{1}$ Udayana University
}

The first cases of rabies in human and dogs in Bali were confirmed in 2008, and now occurs in 161 villages out of 723 villages. Despite control efforts in 2008-2009, rabies spread across the island. In the following 3 years $>130$ persons died from rabies. The aim of this project is to empower Balinese communities group to understand and address concerns regarding dog health and community health by expanding the International Fund for Animal Welfare (IFAW)/BAWA pilot "Participatory Learning and Action (PLA)" program which operates in Sanur regency, Bali. Project Dharma aims to lobby government seeking change in policy and practice; working together with and influencing stakeholders; and engaging communities, including using the media as a channel to influence decision-makers and communities. All to achieve the objectives of the project. The output of pilot project are to: Ensure at least $70 \%$ of all dogs inhibits every pilot project area are vaccinated. The dogs including domestic dogs and stray dogs. All the village member agrees about none of unnecessary dogs elimination or no culling that will sign into a Memorandum of Understanding (MOU). Help communities to make a term of reference and allocate the same program into Village Development Funds

909

\section{Tackling Rabies in Indonesia with a One Health Approach}

\author{
Anak Agung Gde PUTRA ${ }^{1,2}$, I. Ketut Diarmita ${ }^{3}$ and Helen Scott-Orr ${ }^{4}$ \\ ${ }^{1}$ Indonesia Veterinary Epidemiology Association \\ ${ }^{2}$ National Animal Health Commission \\ ${ }^{3}$ Directorate of Animal Health \\ ${ }^{4}$ Sydney University
}

Rabies has been recorded in Indonesia since 1884, gradually spreading from West Java to 25 provinces, although it was eradicated from a further four provinces in the 1990s. Lessons from attempts to eradicate the incursions into Flores since 
1997 and Bali since 2008 have been used to frame a Masterplan for rabies eradication from Indonesia, which, although expensive, will be cost-beneficial in the long term. It relies heavily on strong structural coordination at senior government levels between the Agriculture, Health and Home Affairs Ministries, as well as an integrated dog bite case management system in the field between local health officers and animal health officers, for early detection and prompt treatment to prevent human deaths. Systematic dog mass vaccination campaigns will progressively eradicate rabies from endemic islands or provinces while risk-based surveillance and dog movement controls will prevent further spread to uninfected areas.

\section{Deciphering Transmission of an Environmental Mycobacterial Infection: Is an Ecohealth Approach Plausible?}

Charles Quaye ${ }^{1}$, Lydia Mosi ${ }^{2}$, Charles Narh ${ }^{3}$, Christelle Dassi ${ }^{4}$, Daniele Konan ${ }^{4}$ and Bassirou Bonfoh ${ }^{5}$

${ }^{1}$ Noguchi Memorial Institute for Medical Research

${ }^{2}$ 1. West African Centre for Cell Biology of Infectious Pathogense, University of Ghana, Ghana. 2. Les Département Environnement et Santé, Center Suisse de Recherches Scientifiques en Cote d'Ivoire, Abidjan, Cote d'Ivoire

${ }^{3}$ 1. Parasitology Department, Noguchi Memorial Institute for Medical Research, University of Ghana, Legon, Ghana 2. Les Département Environnement et Santé, Center Suisse de Recherches Scientifiques en Cote d'Ivoire, Abidjan, Cote d'Ivoire

${ }^{4}$ 2. Les Département Environnement et Santé, Center Suisse de Recherches Scientifiques en Cote d'Ivoire, Abidjan, Cote d'Ivoire

${ }^{5}$ Director, Center Suisse de Recherches Scientifiques en Cote d'Ivoire, Abidjan, Cote d'Ivoire

The exact mode of transmission of environmental mycobacteria remains challenging and has negatively affected the implementation of control and preventive strategies. Buruli ulcer (BU) is an example of such a non-tuberculous mycobacterium infection which also lacks adequate information on the survival and transmission of the causative organism, Mycobacterium ulcerans. With an Ecohealth approach, four BU endemic communities in Ghana were mapped and all water contact areas identified. Using structured questionnaires, activities around the water bodies in relation to water contact areas were determined. Viable number tandem repeats (VNTR) profile types of human disease and environmental samples were then compared for infection overlaps. VNTR profiles of fourteen confirmed human BU disease samples and 140 environmental samples were compared. Four assigned human profiles identified W, X, Y and Z were also detected in water contact sites associated with human activities and a higher risk of infection for swimming and bathing $(\mathrm{OR}=3.2840$, $\mathrm{p}=0.045, \mathrm{n}=224)$ was identified. No type was detected in human disease that was not detected in a community associated waterbody suggesting direct human infection from community associated environments through activities that may cause skin abrasions. The multidisciplinary Ecohealth approach is recommended as an effective tool for ecological studies of mycobacterial infections.

808

\section{Multicriteria Analysis Model Based on Ecosystemic Approach to Reduce Territorial Health Inequalities (MARITSA)}

Anne R

National School of Public Health (EHESP)

Despite numerous uncertainties and the complexity of Territorial Health Inequalities (THI), an important challenge for Scientists is the development of a usable decision making tool to implement concrete and targeted action to reduce THI. The objective of the project is to co-construct, thanks to a multidisciplinary network approach, a predictive tool to assess the sensitivity of a territory to produce and accumulate health inequalities (MARITSA). The results of the modelling will 
allow, firstly, to identify the more sensitive territories and, secondly, to target the actions to be implemented in order to reduce the health inequalities. The development of Maritsa is based on a multicriteria decision analysis approach. The tasks are organized around the following mains steps: 1- Problem structuring phase at the territorial scale which is a long process of collection and analysis of data that ends by generating a preliminary set of alternatives (ex. Neighborhood level) and a set of criteria (determinants of health) against which these alternatives are to be evaluated and compared 2- Modelling and results analysis 3- Comparison of the modeling results (ranking of the territories) with a set of « field data " and - 4Application of the model as a decision-making tool.

955

\title{
A One Health Database for Tracking Antibiotic Resistance
}

\author{
Peter Rabinowitz ${ }^{1}$, Scott Weissman ${ }^{2}$, Sally $\operatorname{Trufan}^{1}$ and Marisa D’Angeli ${ }^{3}$ \\ ${ }^{1}$ Center for One Health Research, University of Washington \\ ${ }^{2}$ Seattle Children's Hospital \\ ${ }^{3}$ Washington State Department of Health
}

Antibiotic resistance in bacteria poses a global health threat to humans, animals, and environments, and is therefore a classic One Health challenge. There is a need for improved stewardship of antibiotics in human medicine, animal medicine, as well as agriculture and aquaculture. Despite this, our traditional methods of tracking the emergence of antibiotic resistant strains of bacteria have been fragmented between human and animal sectors, and generally non-existent in the environmental realm. Efforts such as the US National Antibiotic Resistance Monitoring System (NARMS) track certain pathogens in humans and animals, but tend to include only a small number of pathogens, and not routinely produce data reports on a local level. We report on the efforts of a Statewide One Health steering committee to assemble an ongoing database of antibiotic resistance in humans, animals and the environment in order to detect emerging trends and monitor the impact of antibiotic stewardship activities. The database currently includes data from state human and animal health labs, the NARMS system, private veterinary clinics and laboratories, and human hospitals. Data from our pilot efforts to assemble and analyze this database, will be presented, with implications for One Health antibiotic resistance tracking efforts in other regions.

\section{4}

\section{Cardiovascular, Diabetes Risk, Bodily Pain, Health and Wellbeing Among Australian Farmers/Agricultural Workers - Future Challenges for Food Producers}

\author{
Muhammad Aziz Rahman ${ }^{1}$, Susan Brumby ${ }^{1}$, Tracey Hatherell ${ }^{2}$ \\ ${ }^{1}$ National Centre for Farmer Health, Deakin University and Western District Health Service \\ ${ }^{2}$ National Centre for Farmer Health, Western District Health Service
}

Farmers, being disadvantaged by living in remote locations, face different physical and mental health challenges. The crosssectional study aimed to explore health, wellbeing, behavioural, cardiovascular and diabetes risks among Australian farming communities. Study participants were $>18$ years of age, spoke English and were involved/associated with farming. Among 1697 study participants, $62 \%$ were male and $58 \%$ were actively farming. Although health was perceived as good to excellent by $88 \%$ of the participants, $39 \%$ reported moderate to very severe bodily pain and $45 \%$ indicated having moderate to very high stress in the last four weeks. More than two-thirds (73\%) were overweight or obese. Farmers were more likely to eat vegetables/fruits everyday ( $86 \%$ vs. $79 \%$, RR $1.08,95 \%$ CI $1.03-1.14)$ and performed regular physical activities (90\% vs. 79\%, RR 1.15, 95\% CI 1.09-1.21) compared to non-farmers. Current smoking rate was only $6 \% ; 57 \%$ consumed alcohol at 
short term high risk levels at least monthly. Half of them were at risk of developing type 2 diabetes (AUSDRISK12) within 5 years; males (90\% vs. $79 \%$, RR1.14, 95\% CI 1.09-1.20) and farmers (88\% vs. $83 \%$, RR1.06, 95\% CI 1.01-1.11) were at increased risk compared to their counterparts. Interventions focusing on bodily pain, stress, weight, alcohol use and diabetes risk among farmers should be the primary focus for health promotion strategies.

1037

\title{
Genetic Diversity of ESBL Producing E. coli at Human, Animal and Environment Interface
}

\author{
Shahbaz Raza ${ }^{1}$, Aamir $\mathrm{Ali}^{2}$, Peter Schierack ${ }^{3}$ and Mashkoor Mohsin Gilani ${ }^{1}$ \\ ${ }^{1}$ University of Agriculture Faisalabad \\ ${ }^{2}$ National Institute of Biotechnology and Genetic Engineering \\ ${ }^{3}$ Brandenburg University of Technology Cottbus-Senftenberg
}

The snapshot of extended spectrum $\beta$-lactamases (ESBL) bugs is greatly linked with the E. coli in humans, animals and environment and badly affecting the One Health concept. Proposed study was aimed for the detection of genetic diversity between ESBL producing isolates under One Health initiative. A total of 150 samples, including humans $(\mathrm{n}=50)$, cattle $(n=50)$ and environment $(n=50)$, were collected. All samples were cultured on CHROM agar and CLSI standard criteria was used for screening of ESBL E. coli isolates followed by Vitek-2 system for determining the antimicrobial profile of ESBL isolates. Clinically important $b l a_{C T X-M-1}$ was detected by PCR. Phylogrouping and replicon typing was done while BOXPCR and PFGE were performed for detection of clonal group. A total of 29 CTX-M-1 containing ESBLs E. coli were isolated from human clinical isolates (32\%), cattle (18\%) and poultry (8\%). 28/29 isolates were resistant to more than 4 antibiotics. F-1A and F-11 genes were detected after replicon typing while phylogenetic group A and B1 were common. PFGE and BOX-PCR pattern reveals that the isolates are not identical sharing different clonal groups. The results of the study suggest that the ESBL producing E. coli are equally present in each component of One Health.

\section{8}

\section{Response of Zoonotic Diseases to Global Change}

David Redding and Kate Jones

University College London

Microscopy remains WHO gold standard. It is performed in hospitals as a standard method. Through the external quality assessment programs (EQA) in microbiology conducted. One third of the regional health centers are still performing it. The purpose being to control the microscopy diagnosis quality and technicians performance. Thick blood smears, thin blood film, stained slides were performed by the National Reference Center (NRC) for malaria chemoresistance housed in IPCI. A questionnaire was submitted to participants. Including slide code, clinical information about the patient, the result of thick blood smear with parasite density, the result of thin blood film with identification of species and sexual forms and results interpretation. On a total of twenty-four (24) smears stained slides and notwithstanding the parasitemia, only $30 \%$ of correct answers were recorded for P. falciparum identification and none for other species. Parasitemia was approximate. Microscopy identification of parasite being WHO standard method entails a major problem. It requires a high qualified staff which is not available in areas where malaria is endemic. Unfortunately rapid tests are not satisfactory as well. New tools like PCR strip should be evaluated to replace microscopy for a strategic fight against malaria. 


\title{
Achieving Impact Using One Health: The Governance of Leptospirosis in Fiji
}

\author{
Simon Reid ${ }^{1}$, Mike Kama ${ }^{2}$ and Peter Hill \\ ${ }^{1}$ School of Public Health, University of Queensland, Herston, QLD, Australia \\ ${ }^{2}$ Fiji Centre for Communicable Disease Control, Ministry of Health and Medical Services, Fiji
}

Fiji has one of the highest leptospirosis burdens in the Pacific region with over 45 cases per 100,000 population and approximately 31 deaths each year. Leptospirosis is preventable. However, responsibility for control measures for reservoirs (and possible solutions) lies with institutions and individuals outside the health sector. These strategies include education and targeted awareness campaigns for specific risk groups, environmental modification and control in reservoir populations such as rodents and livestock. To address this, in 2013-2014, the Fijian Ministry of Health and Medical Services (MOHMS) initiated the development of a national strategy that involved two multi-sectoral consultation workshops to define issues and identify solutions. Consultation around the national strategy used institutional advocacy to develop a governance structure and interventions to meet the needs of the different stakeholders. A University of Queensland research collaboration, funded by the Bill and Melinda Gates Foundation, undertook a series of interviews with stakeholders to identify measures of disease impact that would facilitate multi-sectoral planning. Despite demonstrable inter-sectoral impact, their conceptualisation of the problem focused only on human morbidity and mortality. Recognising the perceived legitimacy of MOHMS leadership, the final workshop used the research evidence to structure a 5-year multi-sectoral action plan to reduce leptospirosis mortality.

\section{Overconsumption of Energy and Excessive Discretionary Food Intake Inflates Dietary Greenhouse Gas Emissions in Australia}

\author{
Brad Ridoutt $^{1}$, Gilly A. Hendrie ${ }^{2}$, Danielle Baird ${ }^{2}$, Michalis Hadjikakou ${ }^{3}$ and Manny Noakes ${ }^{4}$ \\ ${ }^{1}$ CSIRO Agriculture, Private Bag 10, Clayton South, Victoria 3169, Australia \\ ${ }^{2}$ CSIRO Food and Nutrition, PO Box 10041, Adelaide, South Australia 5000, Australia \\ ${ }^{3}$ UNSW Sustainability Assessment Program, Sydney 2052, Australia \\ ${ }^{4}$ CSIRO Food and Nurition, PO Box 10041, Adelaide, South Australia 5000, Australia
}

Population dietary guidelines are mainly focussed on nutrition and health, but more quantifiable evidence is needed to understand the environmental impacts of food choices. This paper utilised the 2011-2012 Australian Health Survey food intake data and the updated IOPC input-output model to estimate the greenhouse gas emissions (GHGe) of Australian's dietary intake, and compare current patterns of eating which vary in diet quality and GHGe to the recommended diet. The average dietary GHGe were $18.72 \pm 12.06$ and $13.73 \pm 8.72 \mathrm{kgCO}_{2} \mathrm{e} /$ day for male and female adults respectively. The correlation between total energy and GHGe was $r=0.54$ ( $p<0.001$ ). Core foods contributed $68.4 \%$ and discretionary foods $29.4 \%$ to GHGe. Of the core foods, fresh meat and alternatives (33.9\%) and discretionary foods (29.4\%) were the highest contributors. The modelling of current dietary patterns showed the contribution of GHGe from discretionary foods was $121 \%$ greater in the average diet and 307\% greater in the 'lower quality, higher GHGe' dietary pattern compared to the recommended diet. Reducing discretionary food intake would allow for small increases in emissions from core foods (in particular vegetables, dairy and grains), thereby providing a nutritional benefit at little environmental expense. There is synergy between messages of healthy eating and environmental sustainability, so a consistent public health message could be developed. 


\title{
Economic Analysis of Vaccinating Cattle Against Rabies in Bhutan: Preliminary Evidence
}

\author{
Sangay Rinchen ${ }^{1}$, Tenzin tenzin ${ }^{2}$, Susan Cork $^{1}$ and David Hall ${ }^{1}$ \\ ${ }^{1}$ University of Calgary \\ ${ }^{2}$ National Centre for Animal Health
}

Rabies has been eliminated from northern Bhutan but still remains endemic in the south. Elimination programs are challenged by the porous border with India. Cross border animal movement results in rabies outbreaks in cattle and other livestock along the border. While rabies control in dogs is implemented through regular mass vaccination and sterilization, no prevention measures are advocated for cattle. Consequently, outbreaks in cattle are increasingly reported resulting in direct and indirect economic losses due to loss of animals and costs of human post exposure treatment. In this preliminary study, we evaluated the economic benefits of vaccinating cattle in two high risk sub-districts located in southern Bhutan. The benefit cost ratio (BCR) ranged from 2.1 to 9.8 for various case scenarios. We calculated BCR for three scenarios: $\mathrm{BCR}=2.1$ for vaccinating cattle at Government expense; $\mathrm{BCR}=5$ when farmers are willing to bear vaccine costs; and $\mathrm{BCR}=9.8$ when farmers bear vaccine costs and delivery combined with other vaccination campaigns. Therefore, we conclude that vaccinating cattle against rabies can be economically beneficial in high risk areas of Bhutan and thus can be advocated as a measure to prevent rabies in cattle. However, further work is required.

\section{A Decision-Focused Information-Centric Model for Infectious Disease Forecast: An Example of Cholera in Haiti}

\author{
Matteo Convertino ${ }^{1}$ and Ana Riviere Cinnamond ${ }^{2}$ \\ ${ }^{1}$ HumNat Lab-Center for Systems Intelligence and Strategic Design-School of Public Health, Environmental Health Sciences \\ Division and Public Health Informatics Program, University of Minnesota, Minneapolis, MN, USA \\ ${ }^{2}$ Regional Advisor, Eco-Health; IHR, Alert and Response to Epidemics, Water-borne diseases Unit (IR); Department of Commu- \\ nicable Diseases and Health Analysis (CHA); Pan-American Health Organization/World Health Organization (PAHO/WHO)
}

Most current modeling techniques do not cater for other socio-ecological drivers of cholera other than climatic factors; neither produce a portfolio of optimal cost-efficient interventions. We propose a Decision-Focused, Information-Centric, and Technology-prone (DIT) model for cholera forecast and optimal management. The model aims at (i) mapping endemic and epidemic areas, identifying fundamental socio-environmental drivers and their interactions, and determine the universality of these drivers; (ii) identifying potential recurrence time of outbreaks and performing multi-scale predictions over space and time; and (iii) defining optimal controls for decreasing population incidence by quantifying the budget to be allocated to different ecosystem controls that consider health outcomes. The model is constructed on an entropy-/network-based variance decomposition principle. It is built for maximizing prediction accuracy in cholera dynamics. It predicts spatiotemporal weekly patterns of incidence with an average accuracy - for two weeks before the outbreaks occurrence - of 92, 85, 84 and $72 \%$ for the peak timing, geographical distribution, total cases, and peak magnitude, respectively. The model carries a Value of Information that is superior to current models. It enables the prediction of triggering criteria for public health control strategies with increased accuracy. The DIT model is effective for both science and public health decision-making. 


\title{
A Novel Tool to Guide Health Policy Decision-Taking: Disease-Specific Health Vulnerability Indicators to Climate Change
}

\author{
Marilyn Aparicio ${ }^{1}$, Ivar Arana ${ }^{2}$, James Aparicio ${ }^{3}$, Mauricio Ocampo ${ }^{4}$ and Ana Riviere Cinnamond ${ }^{5}$ \\ ${ }^{1}$ Head of the Climate Change, Environment and Health Unit (UCCLIMAS), Instituto Boliviano de Biologia de Altura (IBBA) \\ ${ }^{2}$ Climate Change and Environmental Health Unit (UCCLIMAS), IBBA, La Paz, Bolivia \\ ${ }^{3}$ Museo Nacional de Historia Natural, La Paz, Bolivia \\ ${ }^{4}$ Colección Boliviana de Fauna \\ ${ }^{5}$ Pan-American Health Organization/World Health Organization
}

Social and environmental determinants of health (SDH) are the first acknowledged root-causes of infectious diseases. They are systematically highlighted in $\mathrm{PAHO} / \mathrm{WHO}$ regional prevention and control strategies. Controlling for SDH would reduce disease burden. How can we determine which non-health sectors contribute the most and how much to health vulnerability? No tool has yet been devised to address, from a complex-systems perspective, the degree of responsibility non-health sectors have in disease occurrence. This is particularly important in a climate change scenario. Health Vulnerability Indicators to Climate Change (HVICC) translate the SDH discourse into a tangible tool. Following multi-criteria decision analysis for variable prioritization, a mathematical formula was developed to cater for the direct and indirect vulnerability factors. Two IPCC climate change scenarios were considered. HVICC are fed from secondary data, are diseasespecific, and focus particularly on vector-borne diseases (VBD). Results obtained map, categorize and quantify the degree of responsibility of non-health sectors on vulnerability to a specific disease. The use of HVICC in regional and/or national VBD control strategies would help in swiftly identifying the most vulnerable locations and target technical cooperation actions towards those non-health sectors acknowledged as responsible of such disease-specific vulnerability.

\section{Building a Collaborative Total Health System Approach to Biosecurity Surveillance}

Catherine Robinson and James Butler CSIRO

Biosecurity relies on strong collaborative partnerships between the government, industry and the community. Natural resource managers in remote regions have a particularly important role to play in the detection of pests to protect against emerging infectious diseases. In this paper we outline a 'total system health approach' to translating and integrating local and scientific information to inform surveillance efforts. Using Indigenous communities in Australia and the Asia Pacific region as a case study, we reflect the importance of understanding how different stakeholder groups source and evaluate information, how they manage and use different kinds of information to make decisions, and if and how the partnerships they use can be improved to build total system health approach to biosecurity surveillance.

\section{Breathing Easier: Maximizing the Use of Clean Cooking Technology to Achieve the Sustainable Development Goals}

\author{
Joshua Rosenthal \\ Division of Epidemiology and Population Studies Fogarty International Center, National Institutes of Health
}


Of the 7 million early deaths per year caused globally by air pollution, approximately 4 million are the result of household air pollution, primarily from combustion of solid fuels for cooking in homes in Low and Middle Income Countries. The Global Alliance for Clean Cookstoves has set a goal of introducing clean cooking technology into 100 million homes around the world by 2020, as part of a massive global effort to modernize household energy use. This generally well-conceived effort dovetails with at least five of the Sustainable Development Goals, including health, environment, and social empowerment objectives. However, most clean cooking programs around the world are still driven by climate change mitigation objectives and financed, at least in part, by carbon credits. The result is that the overwhelming majority of the nearly 49 million households that have participated since the goal was established in 2010 are using relatively low-tech improved cookstoves that burn solid fuels more efficiently, but yield minimal health benefits. A strategic focus on clean fuels, such as liquefied petroleum gas, biogas or ethanol, rather than improved biomass stoves will provide significantly greater benefits to all five relevant Sustainable Development Goals.

307

\title{
One Health Concerns in Social-Ecological Systems and Opportunities for Building Resilience
}

\section{Helen Ross}

The University of Queensland

Social-ecological systems are considered as 'coupled' human and ecological systems, in which people and their environments interact at multiple scales, individual to global. In these complex adaptive systems, processes are not linear, and there is no 'control' or 'determinants'. Rather, one seeks to understand important patterns and risks, and to build adaptive capacity and resilience. This presentation canvasses the opportunities of thinking about One Health concerns in a socialecological systems way, and what a 'resilience' goal might entail. It canvasses the influences of globalisation, which has altered people's customary relationships with wild animals, livestock and companion animals through increased world trade and travel and land clearing; and of poverty, which can involve high dependence on natural resources, high vulnerability to disease and low resistances, high impact of control measures and particular communication challenges. Ecological resilience focuses on the ability to withstand shocks, and social resilience on the strengths that assist a society to do so. What cultural, cognitive and institutional strengths do we need to consider to build resilience and adaptive capacity in relevant societies and environments, and in One Health's preparation and response systems?

792

\section{Field Studies on Aestivation In Umkhanyakude District of South Africa}

\author{
Owen Rubaba, Moses Chimbari and Samson Mukaratirwa \\ University of KwaZulu Natal
}

Schistosomiasis transmitting intermediate host snails survive drought periods through aestivation. It is envisaged that with climate change dry/wet seasons may become shorter or longer, thereby reducing or increasing their chances of survival. This study investigated the ecology of the snails in four rivers in uMkhanyakude district to determine the influence of draught duration on snail survival and breeding. Aestivation patterns in Biomphalaria pffeifferi and Bulinus globosus were studied by first monitoring changes in snail population structure during the period when the habitats were flooded and then digging transects across the floor of their dried habitats at specified points for 12 months. A wide size range for both species was 
found to aestivate. Successfully aestivated snail numbers significantly declined for the first 3 months after the drying of the river beds. The longest period of aestivation for B. globosus snails was 8 months while that of B. pfeifferi was 6 months. More snails were found at the river banks compared to the centre of the riverbeds suggesting they preferred sheltering themselves among the vegetation and did not necessarily follow the receding waters. This study showed that aestivation ability declines with time and occurred in the first $5 \mathrm{~cm}$ from the surface.

\title{
The First Interdisciplinary MOOC (Massive Open Online Course) on Global Health at the Human-Animal-Ecosystem Interface
}

\author{
Rafael Ruiz de Castaneda, Isabelle Bolon and Antoine Flahault \\ Institute of Global Health, Faculty of Medicine, University of Geneva
}

MOOCs bring a new dimension to teaching and learning. Some of world's best Universities use MOOCs to share their knowledge with a global community of learners, opening unprecedented opportunities for career development. MOOCs are increasingly used for dissemination of policy recommendations, as seen during the climate crisis or the Ebola epidemic. The Institute of Global Health (IGH) has produced several MOOCs with an innovative interdisciplinary approach, part of International Geneva's identity (e.g. MOOC on Ebola involved 38 experts from 20 institutions). This approach has a potential added value in terms of the diversity of roles and perspectives needed for these complex global health challenges. In December 2016, we launch on Coursera a new MOOC on Global Health at the Human-Animal-Ecosystem Interface: Interdisciplinary Overview. It is co-produced by the IGH and Institut Pasteur involving also a diversity of experts from other academic institutions, international organisations (e.g. WHO) etc. It will be free and open, targeting primarily an interdisciplinary audience interested in human-animal-ecosystem health interface. Besides its primary educative objective, the MOOC will become a platform for interdisciplinary discussions, encounters online but also offline in different parts of the world (e.g. Meetup, Hackathon), as well as participatory research (Massive Open Online Research).

\section{Using Ecohealth Approaches To Identify Health Challenges: Case Study Of Communities In and Around Queen Elizabeth National Park}

Innocent Rwego ${ }^{1}$, Benon Asiimwe ${ }^{2}$, Catherine Kansiime ${ }^{3}$, Katey Pelican ${ }^{4}$, Cheryl Robertson ${ }^{4}$, Thomas Gillespie ${ }^{5}$, Dominic Travis ${ }^{4}$ and Joel Hartter ${ }^{6}$

${ }^{1}$ University of Minnesota and OHCEA, Makerere University

${ }^{2}$ Makerere University College of Health Sciences

${ }^{3}$ One Health Central and Eastern Africa-Makerere University School of public Health

${ }^{4}$ University of Minnesota

${ }^{5}$ Emory University

${ }^{6}$ University of Colorado

One of the greatest challenges to improving health in Africa has been a lack of long-term and systemic engagement of various stakeholders in vulnerable communities. Interventions are often vertical and intermittent and, often instigated by a crisis. Community capacity is impeded by persistent exposure to complex public health problems not addressed by vertical interventions, including food and water insecurity, the effects of climate change, unsustainable natural resources, and political conflict and mobile populations. The One Health Central and Eastern Africa (OHCEA) network of universities is building on this model of long-term engagement to establish a regional network of One Health Demonstration Sites in six 
countries to address complex health problems faced by communities. The presentation will show community identified priorities and ranked for future interventions in Queen Elizabeth National Park (QENP) in Uganda. Priorities were identified using an Ecohealth approach whereby communities participated in identifying, ranking and prioritizing health and environmental challenges in their population(s), as tell as the variables that drive poor health in humans and animals and/or contribute to environmental degradation. In QENP, traditional pastoralist communities have been forced to become semi-sedentary and, in some places, completely displaced and forced into national parks and other conservation areas because of lack of pastures, watering points and loss of land to agriculturists. Fishing communities have stayed in and around national parks to exploit lakes within these conservation areas. This has led to a perceived increase in humanwildlife conflict in these areas. Interaction between wildlife- domestic animals and humans is relatively higher within fishing enclaves than in surrounding communities. This scenario leads to increased zoonotic diseases, environmental degradation and global change.

635

\section{Zoonotic Tuberculosis in Cattle, Goats and Humans in Bangladesh, 2014-2015 Using a One Health Approach}

Shamim Sarkar ${ }^{1}$, Najmul Haider ${ }^{1}$, Ariful Islam ${ }^{1}$, James D. Heffelfinger ${ }^{2}$, Kamal Hussain ${ }^{1}$, Zeaur Rahim ${ }^{1}$, Nordin S. Zeidner ${ }^{2}$

${ }^{1}$ Programme on Emerging Infections, Infectious Diseases Division, icddr, $b$, Dhaka, Bangladesh

${ }^{2}$ Centers for Diseases Control and Prevention, Atlanta, GA, USA

The objective of this study was to determine the prevalence of Mycobacterium bovis in animals and humans in Bangladesh. We collected blood and tissue samples from cachectic cattle and goats from August 2014 through September 2015 from two abattoirs and a veterinary hospital. We collected sputum from workers and traders at a cattle market and from patients at two government chest diseases hospitals who had history of animal exposure. Blood specimens were tested by ELISA for antibodies to M. bovis. Animal tissue and sputum specimens were screened for acid-fast bacilli (AFB) by Ziehl-Neelsen staining and cultured for Mycobacterium spp. Overall, 393 animals and 412 humans were sampled. No animal specimens were positive for M. bovis antibodies, AFB or Mycocobacterium spp. Twenty-five (6\%) human sputum specimens were positive for AFB, 43 (10\%) for M. tuberculosis and none for other Mycobacterium spp. No evidence of current or past infection with $M$. bovis in animals or humans was found. No evidence of M. bovis may be due to sampling mostly indigenous/native animal which are less susceptible to $M$ bovis infection than exotic. Further assessment of zoonotic TB in areas of Bangladesh with high levels of exotic animal-human interaction and human TB is important.

937

\section{Addressing the Emergence and Dispersion of Leishmaniasis in the Border of Argentina, Brazil and Paraguay and Uruguay}

Oscar Daniel Salomon ${ }^{1}$, Vanete Thomaz-Soccol ${ }^{2}$, Nilsa Gonzalez Britez ${ }^{3}$, Gabriela Willat Real de Azua ${ }^{4}$ and Zaida Estela Yadon ${ }^{5}$

${ }^{1}$ National Institute of Tropical Medicine MOH, Argentina/CONICET

${ }^{2}$ Universidade Federal do Paraná

${ }^{3}$ Institute of Health Sciences, National University (IICS-UNA)

${ }^{4}$ Ministry of Health

${ }^{5}$ Pan American Health Organization 
Cutaneous leishmaniasis (CL) and visceral leishmaniasis (VL) are vector-borne zoonotic parasitic diseases, with more than 2,000,000 new cases/year worldwide. The current control measures have relatively low effectiveness or there are culturally or ecologically unsound. This project look for the social and biological drivers of risk on the Argentina, Brazil and Paraguay border, and Uruguay (IDRC-PAHO). The locations and domestic units visited were around 500 to perform sampling of vectors, dogs, rodent activity, ecological surveys and social interviews. The main outcomes were: (1) The characterization of VL spread and CL risk, with parasite genotypification from humans, vectors, and animal reservoirs (dogs). (2) The spatial segregation of the vectors of each leishmaniasis, associated with variables that could allow the modelling of its distribution in time and space. (3) The evidence-based delimitation of an area for environmental interventions of $400 \times 400 \mathrm{~m}$. (4) The social modulators of risk, mainly of VL and canine VL, due to the human-dog inter-specific relationship and consequent ways of management. Currently we are integrating the multidisciplinary results an to promote an intersectoral discussion in order to develop alternative and feasible strategies of surveillance, prevention and control of leishmaniases in a healthy environment.

\title{
Small Ruminants, Household Food Security and Gender Dynamics: Insights from the Gambia
}

\author{
Isatou Savage, Siobhan Mor ${ }^{1}$, Darryl Stellmach ${ }^{2}$ and Robyn Alders ${ }^{3}$ \\ ${ }^{1}$ The university of Sydney \\ ${ }^{2}$ University of Sydney
}

In the Gambia, undernutrition in children remains problematic with 23.4 percent of children being moderately or severely stunted. Sixty percent of the population live in rural areas and are dependent on agriculture for their livelihoods. The raising of small and large ruminants, donkeys and village poultry is crucial due to poor cropping conditions. Generally, women have responsibility for small ruminants and village poultry with men taking most interest in large ruminants and donkeys. In a mixed methods study of a survey and focus group discussion, involving 120 households in the Kiang East District, competition between livestock species for pasture, fodder and crop residues was well recognised. Our research also highlighted the associated competition between men and women in relation to which animals could access crop residues and how these animals were used within households. Both men and women tended to rear small ruminants for the purpose of household consumption and finance. Other problems raised by women include inadequate access to grazing land, lack of capital to purchase feed supplements and lack of adequate know-how. Our findings demonstrated that despite women 's significant role in small ruminant production, they still lack adequate support to improve their contribution to household food security.

\section{Evaluating a Decade of Australia's Investments in Pandemics and Emerging Infectious Disease Preparedness and Response in Asia Pacific: Are Health Systems Stronger?}

\author{
Irene Wettenhall ${ }^{1}$, Gill Schierhout ${ }^{2}$, Adam Craig, David Slattery ${ }^{1}$ and Laurence Gleeson ${ }^{1}$ \\ ${ }^{1}$ Department of Foreign Affairs and Trade \\ ${ }^{2}$ Kirby Institute, University of NSW
}

There is more to be learned about how aid programs focused on pandemics and emerging infectious diseases (PEID) influence country health systems, both animal and human and the interface between them. This paper presents findings from a major evaluation conducted by the Department of Foreign Affairs and Trade (DFAT) of Australia's efforts to reduce 
the risk of PEIDs across Asia and the Pacific between 2006 and 2015. Led by DFAT's Office of Development Effectiveness, the evaluation covered 30 initiatives, worth over $\$ 190$ million and included data collected from fieldwork in Indonesia, Cambodia, Thailand, Fiji, Solomon Islands and Australia. Drawing on current understandings of health systems strengthening and on the WHO's International Health Regulations and OIE Performance of Veterinary Services, we assess the impact of these investments on country health systems. By exploring implementation characteristics and outcomes achieved in different contexts, we identify 'what works for whom and in what circumstances' in building stronger health systems, and linkages between animal and human health systems. Implications of the findings for future investments in this area will be discussed.

692

\title{
Green Space Versus Wild Space: Are Some Types Of Nature Better for Wellbeing than Others?
}

\author{
Jacki Schirmer, Brigitta Yabsley \\ University of Canberra
}

Internationally there is growing investment in improving human wellbeing through interactive nature experiences. However, while there is a strong body of evidence showing positive linkages between spending time in urban green spaces or natural (wild) spaces and a person's wellbeing, some studies have also found limited connection. Concerns have also been raised that factors such as fear and lack of knowledge about being in nature may act to moderate the potential benefits of spending time in either green spaces or wild spaces. This paper examines evidence from the 2014 Regional Wellbeing Survey, in which 5,000 Australians were asked about the types of green spaces and natural spaces they spent time in, and whether they experienced any of a number of factors considered likely to moderate or mediate the wellbeing benefits of spending time in these places. It focuses in particular on identifying whether spending time in green spaces such as urban parks has different associations with wellbeing compared to spending time in wild spaces such as national parks and wilderness areas.

695

\section{Are Alternative Agricultural Systems Good for Wellbeing as well as for Environmental Sustainability? Evidence from Australian Farmers}

Jacki Schirmer, Kimberly Brown

University of Canberra

In Australia and internationally, growing numbers of farmers are managing their land using alternative agricultural methods intended to increase the sustainability of agriculture. Going under many labels, including biodynamic, organic, regenerative, holistic and natural sequence farming, these farming practices are argued to be better not only for the environment but also for the farmer. While the environmental benefits of these practices have been established to some extent, the claims made regarding the benefits of these farming practices for the wellbeing of agricultural households have not. This paper presents a quantitative analysis of the wellbeing of farmers engaging in these practices, based on a survey of 3,000 Australian farmers conducted as part of the annual Regional Wellbeing Survey. It examines whether alternative farming practices are associated with improved health and wellbeing for farmers compared to traditional farming practices, 
and in what circumstances. Based on this, the authors proposed a new approach to classifying alternative agricultural practices that focuses not only on their environmental outcomes but which uses a socio-ecological systems approach to classify them based on social, economic and environmental outcomes.

\title{
Predictive Modelling of the Change in Spatial Distribution of Japanese Encephalitis in Nepal Due to Climate Change, and the Benefit of Modifying National Control Policies in Response
}

Barun Kumar Sharma ${ }^{1}$, Swagat Nepal ${ }^{1}$, Binod Prasad Gupta ${ }^{1}$, Ishwari Prasad Dhakal ${ }^{2}$, Minu Sharma ${ }^{3}$, Sithar Dorjee $^{4}$, Joanna McKenzie ${ }^{4}$, Peter Jolly ${ }^{4}$, Masako Wada ${ }^{4}$ and Roger Morris ${ }^{5}$

${ }^{1}$ One Health Epidemiology Postgraduate Fellow, Massey University

${ }^{2}$ Agriculture and Forestry University

${ }^{3}$ National Zoonoses and Food Hygiene Research Center

${ }^{4}$ Institute of Veterinary Animal and Biomedical Sciences, Massey University, New Zealand

${ }^{5}$ Morvet Ltd, Consultancy services in health risk management and food safety policy and programs, Masterton, New Zealand

A vaccination program targeting children has reduced the incidence of Japanese encephalitis (JE) in endemic areas of Nepal. However JE risk is expanding from the lowland terai regions into higher altitudes, exposing new human populations to the mosquito vector, Culex tritaeniorhynchus. Pigs are important amplifying hosts, but their distribution within Nepal is influenced by the religious and ethnic composition of local communities. Movement of viraemic pigs into areas where there is sufficient density of the mosquito vector may contribute to the expansion of JE risk areas. Spatial data on pig density, altitude, precipitation, humidity, temperature and land use were combined to create a JE risk map which identified the expanded areas in which JE was likely to occur. The variation in risk was then used to adjust disease transmission in a spatially explicit stochastic model of JE in HandiSpread, which models virus transmission in pigs and vectors, and the exposure of people to the virus. Model parameters were informed through a social network analysis of pig movements conducted in an example area of Nepal where risk of JE is increasing. Cost-effectiveness analysis was used in conjunction with epidemiological predictions from the model to assess potential changes in current control policies.

374

\section{Local Ngo in Nepal Builds Capacity in Rabies Prevention Using One Health Approaches}

\author{
Khageshwaar Sharma, Barbara Webb and Jim Pearson \\ Himalayan Animal Rescue Trust
}

The control and prevention of rabies in Nepal is fragmented and requires improved coordination and enhanced access to post exposure prophylaxis in rural areas. A local non-government organisation, Himalayan Animal Rescue Trust (HART) has set up bases on the premises of the District Livestock Service Office (DLSO) in Pokhara and Bharatpur to generate interest from the authorities, whose primary concern is livestock, in rabies prevention and dog population management. Mobile phone applications have been developed to enable dog census and mass anti-rabies vaccination data to be captured and assessed, ensuring over seventy percent of the dog population are rabies vaccinated. Currently HART is working with the Nepalese Animal Health Directorate and Veterinary Public Health Office to transfer this field surveying technology and expertise to local DLSO's enabling 
rabies prevention programs to be coordinated by the government. Additionally, World Veterinary Services are working with HART to train Nepalese veterinary students in companion animal sterilisation surgery, building capacity in population management and animal welfare. HART has considerable experience and operational knowledge in rabies control which can help both the human and animal health sectors to build a National Rabies Strategy for Nepal to eliminate dog-mediated rabies.

\title{
Multidisciplinary Teams Bring Help and Hope to Farmers in Nepal Following the 2015 Earthquakes
}

\author{
Khageshwaar Sharma ${ }^{1}$, Catherine Sutton ${ }^{2}$ and Andrea Britton ${ }^{3}$ \\ ${ }^{1}$ Himalayan Animal Rescue Trust \\ ${ }^{2}$ Cobram Veterinary Clinic \\ ${ }^{3}$ Ultimate Efficacy Consulting Pty Ltd
}

Immediately following the 25th April 2015 major earthquake in Nepal local non-government organisation (NGO), Himalayan Animal Rescue Trust, suspended all rabies associated programmes to help communities in devastated districts. One base focused on Dhading, Nuwakot, Lamjung and Dolakha districts reaching 164 farmers during May and the second base focused on Gorkha and Sindupalchowk reaching 322 farmers between May and June. Australian and New Zealand veterinarians came to assist providing medicines and expertise especially in treating fractures and recumbent cattle. Multidisciplinary teams consisting of: driver, first aid personnel, local NGO and overseas veterinarian, were formed and went to the worst affected remote villages, at times walking to gain access. The teams were often the first help to arrive, handing out food, clothing and delivering veterinary care. Over-all 1531 animals were treated including multiple fractures in 18 cattle and buffalo, and rabies vaccinating dogs. Livestock are the mainstay of subsistence farmer's economies so the teams brought hope to families where every animal is precious. The Sindupalchowk district where the second earthquake hit was visited four times to recheck cases with many recovering due to twenty-four-hour care given by farmers. Contingency planning with appropriate multidisciplinary teams is recommended for post-disaster responses.

\section{0}

\section{Challenges and Opportunities for Food Security and Health in Rural Africa}

\section{Elizabeth Shayo \\ National Institute for Medical Research}

Food security is achieved, when adequate food (quantity, quality, safety, socio-cultural acceptability) is available and accessible for and satisfactorily utilized by all individuals at all times to live healthy and happy life. It has been revealed that food security assurance and balanced diet uptake translate into a healthy population. If these conditions happen, the healthy population is assured of sufficient growth, ability of their bodies to fight or recover from diseases and other health related conditions. However, there are challenges facing poor households today. Immense literature reveals prevalence of problems associated with food insecurity including their impacts on health. Food security in Sub Saharan Africa especially in rural areas has worsened since 1970 with about 33-35\% of the population being malnourished. Factors attributed to food insecurity include poor agricultural practices, barriers to market access, effects of globalization, diseases and infections and poor policies. Improving agricultural practices, increasing the agricultural profitability of smallholder farmers and creating rural off-farm employment are opportunities to address the gaps. Further attention need to be paid on the underlying 
socio-cultural and contextual drivers and inequities in conditions of daily living that dis-empower individuals from engaging in activities that address food insecurity and malnutrition in an effort to enhance good health. The discussion will help to inform policy makers to develop or improve the existing policy on food security and nutrition, and further help decision makers to take appropriate action.

\title{
Antimicrobial Resistance Threat in Indonesia - A Need for One Health Approach
}

\author{
Selma Siahaan ${ }^{1}$, Rini S. Handayani ${ }^{1}$, Max J. Herman ${ }^{1}$, Retno Gitawati ${ }^{1}$, Siti Isfandari ${ }^{1}$ and Oce Boymau ${ }^{2}$ \\ ${ }^{1}$ National Institute of Health Research and Development \\ ${ }^{2}$ East Nusa Tenggara Province Health Office
}

There is high rates of antimicrobial resistance (AMR) in Indonesia. This situation is caused by inappropriate and irrational use of antimicrobials. A study to examine and assess AMR policy in Indonesia had been conducted from October 2014 to April 2015 in 8 provinces, representing the capital, west, central and eastern part of Indonesia. It covers systematic review of documents on study results and policies, data collection of the most frequently used antimicrobials in health care facilities, in-depth interview with government officers from health, livestock and fisheries sectors regarding the use of antimicrobials for humans, animals and fishes. The results show that AMR situation tends to be deterioting. Amoxicillin is the most widely and frequently used products, either in health cares, farms and communities. The current policies related to managing AMR in each sector cannot solve the AMR problems. There was lack coordination among related sectors contribute to AMR situation in Indonesia. The study recommends that Indonesia needs a "One Health Approach" to directly address the AMR situation. The Government should issue the "National linkage policy on AMR" to foster collaboration among the different sectors and for it to be integrated into regional policies.

1050

\section{Vector-borne Co-infections in an Intensive Human-livestock-wildlife Interface Ecosystem in Northern Tanzania}

\author{
Mary Simwango $^{1}$, Sharadhuli Kimera ${ }^{1}$ and Paul Gwakisa ${ }^{2}$ \\ ${ }^{1}$ Department of Veterinary Medicine and Public Health, Faculty of veterinary Medicine, Sokoine University of Agriculture \\ (SUA), Morogoro, Tanzania \\ ${ }^{2}$ Department of Veterinary Microbiology and Parasitology, Faculty of Veterinary Medicine, Sokoine University of Agriculture, \\ Morogoro, Tanzania
}

The Maasai steppe ecosystem at the wildlife/ livestock/human interface is known to support occurrence of vectors that transmit pathogens to livestock and vulnerable communities in northern Tanzania. The transmission of these pathogens result in increased incidence of vector-borne diseases causing serious livestock and economic losses to the Maasai people. This study aimed at establishing the extent to which human activities affect the abundance of three protozoal pathogens in the Maasai steppe. Polymerase Chain Reaction was performed to detect 3 vector-borne parasites of importance in blood collected from 200 cattle of known East Coast fever (ECF) vaccination status in 2 villages of Simanjiro district. The overall prevalence of blood parasites was $29.5 \%$, of which $16.5 \%$ were infected with trypanosomes, $12.5 \%$ with Theileria parva and 
$4 \%$ with Ehrlichia ruminantium. The study also revealed that ECF vaccinated cattle were $43 \%$ less likely to carry trypanosomes. $11.9 \%$ of infected cattle carried concurrent infections with 2 parasites and only calf carried all three parasites. This study provides evidence to the risk associated with vector-borne diseases in the Maasai steppe ecosystem and suggests importance of comprehensive control measures directed at ecology and transmission of pathogens to livestock and humans in wildlife interface areas of northern Tanzania.

483

\title{
Climate Change and Malaria Transmission in Korhogo
}

\author{
Pewonheta Dramane Soro ${ }^{1}$, Kouassi Richard $\mathrm{M}^{\prime} \mathrm{bra}^{1}$, Brama Kone ${ }^{1}$ and Nagnin Soro ${ }^{2}$ \\ ${ }^{1}$ Centre Suisse de Recherches Scientifiques en Côte d'Ivoire \\ ${ }^{2}$ Université Félix Houphouet Boigny
}

The city of Korhogo in northern Côte d'Ivoire, has suffered during the last decade the throes of climate. It recorded a severe drought between 2004 and 2005 and torrential rains in 2006, causing extensive damage. The present study aims to access the influence of these variations on malaria transmission. Meteorological data were extracted from the database of the International Research Institute for Climate and Society. As for the malaria data, they were collected in public and private health facilities in the city of Korhogo. The analysis of rainfall and temperature data related to the number of malaria cases permit to establish the link between climate and malaria. The correlations $(r)$ are relatively average between rainfall and the incidence of malaria of same month $(r=0.30)$, of first month $(r=0.42)$ and of second month following $(r=0.50)$. The registered determination coefficients $\left(R^{2}\right.$ between $9 \%$ and 25\%) indicate that in addition to the climate, other factors are involved in malaria transmission. The temperature maintains a negative relationship with malaria and explains $10.89 \%$ of malaria cases $\left(r=-0.33\right.$ and $\left.\mathrm{R}^{2}=10.89 \%\right)$. These observations could help decision makers anticipate the importance of mortality attributed to malaria in Korhogo.

851

\section{Large-Scale Soybean Production in the Amazon and Pesticide Use: Ecosystem and Health Concerns}

\author{
Carlos Jose Sousa Passos ${ }^{1}$, Bruna Maria Silva Caldas ${ }^{1}$ and Juliana Valentini ${ }^{2}$ \\ ${ }^{1}$ Faculty UnB at Planaltina, University of Brasilia \\ ${ }^{2}$ Faculty of Health Sciences, University of Brasilia
}

This study assesses human exposure to pesticides at intensive soybean production areas, located in the Brazilian Amazon rainforest. With a cross-sectional study design, we measured the enzyme activity for plasmatic butyrylcholinesterase (BChE) as biomarkers of human exposure, at local and small-scale agricultural households living around huge soybean crops, during two key sampling periods: February 2015 (fumigation period) and August 2015 (non-fumigation period). Plasmatic BuChE activity was determined by Doles ${ }^{\circledR}$ commercial kits, following Brazilian Regulations. A socio-demographic, environmental, health, and occupational questionnaire was applied to 82 villagers, both adult men and women, who read and signed an informed consent form. BuChE activity averaged 9.4 $\pm 2.7 \mathrm{UI} / \mathrm{mL}$ in August (median: 9.2; range: 3.0-15.8 $\mathrm{UI} / \mathrm{mL}$ ) and $5.4 \pm 1.9 \mathrm{UI} / \mathrm{mL}$ (median: 5.0; range: 1.7-11.7 UI/mL) in February. Paired comparative analyses for a subsample of 38 persons indicate an averaged 39.2\% BuchE inhibition (range: 0-80\%), where 12 persons (31.6\% of the 
subsample) present $\mathrm{BuChE}$ inhibition $\geq 50 \%, 27$ persons (71.1\%) show BuChE inhibition $\geq 30 \%$, and 29 persons $(76.3 \%)$ reveal BuChE inhibition $\geq 25 \%$, with many study participants self-reporting toxic symptoms typical of acute pesticide exposures. Our data suggest risks for ecosystem contamination and human exposure to pesticides in the context of large soybean production in the Brazilian Amazonia.

\title{
Global Health and Environmental Implications of Informal Extractive Industries: Insights from the 2010 Outbreak of Severe Acute Lead Poisoning in Northern Nigeria
}

\author{
Darryl Stellmach \\ University of Sydney
}

This presentation employs a qualitative One/EcoHealth perspective to examine long-term global public health implications of artisanal mining and other informal extractive industries in remote or resource-poor settings. In 2010, an outbreak of lead poisoning among artisanal mining communities in Northern Nigeria lead to the deaths of approximately 400 children, and permanent sequelae in large segments of the population. Recognized as the most severe acute mass lead poisoning in recorded medical history, the outbreak is a signal incident that illustrates the broader health and ecological implications of small-scale mining and other informal industrial practices done in situations of economic and social marginality. This presentation provides a summation of the outbreak and the public health and environmental insights that emerged. In recent years, rising international prices for precious minerals have led to a global boom in informal extractive industries. While sometimes portrayed as entrepreneurial and emancipatory, these activities occur at the margins of governmental influence, with little attention paid to personal or environmental protections and often in a context of exploitation. These industrial hazards can be subtle and pervasive, thus the health and environmental effects for individuals and societies can unfold imperceptibly, to emerge on a global and generational scale.

\section{Living Off the Land - The Role of Wild Foods in Food Security}

\author{
Eloise Stephenson ${ }^{1}$, Philip Thornton ${ }^{2}$, Jessica Bogard ${ }^{3}$, Cecile Godde ${ }^{3}$ and Mario Herrero ${ }^{4}$ \\ ${ }^{1}$ CSIRO/Griffith University \\ ${ }^{2}$ CCAFS/ILRI \\ ${ }^{3}$ CSIRO/University of Queensland \\ ${ }^{4}$ CSIRO
}

It is estimated that more than a billion people rely on wild foods as a source of protein, nutrients and income, all contributing to food security. However, because wild foods are not considered food commodities, and therefore don't appear in food balance sheets, their contribution to food security is often overlooked. We reviewed $>80$ studies focusing on wild foods across agriculture, nutrition, social and environmental disciplines to identify how wild foods contribute to the four pillars of food security (availability, access, utilisation and stability). We assessed the location (i.e. country, climatic zone), methodology (i.e. survey, observational) and motivation (i.e. conservation, human health) for each study to identify trends in wild food research. Wild foods directly contribute to food security, particularly 'stability'. Wild foods also indirectly contribute to food security in a number of ways which are synonymous with One Health, such as biodiversity improving nutritional security and human health outcomes. Presently, our understanding of wild foods in a food security context is limited by lack of data on harvest/production, consumption/dietary habits and nutritional value of many wild 
foods. We propose a multidisciplinary framework to address these gaps, allowing better assessments of wild foods and food security in the future.

\title{
210
}

\section{Humoral Immune Responses of Rousettus Aegyptiacus to Marburg Virus}

\author{
Nadia Storm ${ }^{1}$, Petrus Jansen van Vuren ${ }^{1}$, Wanda Markotter ${ }^{2}$ and Janusz Paweska ${ }^{1}$ \\ ${ }^{1}$ Centre for Emerging and Zoonotic Diseases, National Institute for Communicable Diseases of the National Health Laboratory \\ Service \\ ${ }^{2}$ Zoonoses Research Unit, Department of Medical Virology, Faculty of Health Sciences, University of Pretoria
}

The Egyptian fruit bat, Rousettus aegyptiacus, is a reservoir host for Marburg virus (MARV). Characterising the antibody responses of reservoir hosts may provide insights into which antibodies may be important for protection against infection, and which proteins to target for diagnosis and surveillance. Additionally, determining when juvenile bats lose maternal immunity may assist in predicting spill-over events into human and animal populations, as loss of immunity in juveniles increases susceptibility of bat populations to MARV infection. We determined the serological responses of Rousettus aegyptiacus to structural MARV proteins using enzyme-linked immunosorbent assays. In experimentally infected bats, antibodies against the glycoprotein (GP) steadily waned after peaking at 12 days post-infection, while antibodies against the nucleoprotein (NP) reached a plateau between days 12 and 42 post-infection. Results in field collected sera showed the presence of anti-NP antibody in the absence of anti-GP antibody, inferring that anti-GP immunity might have waned below detectable levels at the time of sample collection. The nucleoprotein might therefore be a better immunoreagent for surveillance studies aimed at assessing past exposure to MARV in Rousettus aegyptiacus. In juveniles, maternal anti-GP and anti-NP antibodies were lost within 3 to 5 months after birth. This information will assist in predicting and preventing future spill-over events.

\section{Understanding Historical and Environmental Change for Better Coastal Adaptation Policies Today}

\author{
Celine Surette $^{1}$, Gregory Kennedy ${ }^{1}$, Omer Chouinard ${ }^{1}$, Serge Larochelle ${ }^{2}$ and Alain Patoine \\ ${ }^{1}$ Université de Moncton \\ ${ }^{2}$ Groupe de développement durable du Pays de Cocagne
}

Using a transdisciplinary framework leveraging natural sciences, social sciences, history and art-based approaches towards integrated policy recommendations, we are studying adaptation to climate change in the Cocagne River Watershed communities, New Brunswick, Canada. The inhabitants have demonstrated exceptional resilience through the creation of local non-governmental organizations and the building of rural development plans. By partnering with these groups, we are working together to develop adaptation strategies to tackle climate change and community health. One approach we use is to combine archival and geochemical analysis to reconstitute the evolving communities of the Cocagne River Watershed from the beginning of industrialization to the present day. Specifically, we have reconstituted the local population with the Canadian Censuses and sampled aquatic sediment cores in order to reconstruct ecosystem changes through indicators of aquatic productivity and the presence of metals. Thus, we use two types of data (archival and biogeochemical) towards the same goal of understanding how land use practices impact ecosystem health and community resilience. Our findings 
highlight the vulnerability and the resilience of small communities in constant change. Better understanding of past adaptations as well as local culture allow us to propose specific coastal adaptation policies to increase health and wellbeing of communities.

\title{
Making One Health Work Across Sectors: Scale And Hierarchies in Indonesia
}

\author{
Pebi Suseno ${ }^{1}$, James McGrane ${ }^{2}$, I. Ketut Diarmita ${ }^{1}$, Elly Sawitri ${ }^{2}$, Muhammad Azhar $^{1}$ and Luuk Schoonman ${ }^{2}$ \\ ${ }^{1}$ Directorate of Animal Health, MoA, Indonesia \\ ${ }^{2}$ FAO ECTAD Indonesia
}

Since 2006 FAO has partnered with the Government of Indonesia (GOI) to implement programmes to control highly pathogenic avian influenza (HPAI). The autonomous nature of Indonesia's sub-national government administrations since 2001 required novel engagement methods to ensure local ownership of central government-led animal disease control programmes. The participatory disease surveillance and response (PDSR) system was established in 31 of Indonesia's 34 provinces to detect, report and respond to HPAI. A One Health $(\mathrm{OH})$ approach was successfully fostered between animal health PDSR staff and human health service district surveillance officers to collaborate in joint investigation of HPAI outbreaks and monitor human exposuresnear animal cases; however lack of sufficient district animal health staff hampers this. While cross-sectoral engagement worked well at the district and sub-district level, the collaborative relationship proved more challenging at the higher provincial and central government hierarchical levels. The function of coordinating zoonotic disease control across line ministries was assigned to a National Committee on Pandemic and Avian Influenza/zoonoses control. In order to make One Health work more effectively in Indonesia closer hierarchical alignment of the Ministry of Agriculture and Ministry of Health zoonotic disease control entities and higher investment in veterinary authority are required.

552

\section{Rabies Awareness Rising for Children in Nias Island, Indonesia: Investment for the Future}

Pebi Suseno $^{1}$, Wahid Husein ${ }^{2}$, Ernawati Ernawati ${ }^{1}$, Sarah Jayme ${ }^{2}$ and I. Ketut Diarmita ${ }^{1}$

${ }^{1}$ Directorate of Animal Health, MoA, Indonesia

${ }^{2}$ Global Alliance for Rabies Control

Rabies was confirmed in Nias Island, North Sumatera, Indonesia in 2010. This incident caused a public concern due to the huge impact of rabies to the community. Up to present 61 human deaths was recorded in 5 (five) districts in Nias Island, and many of that cases occurred in children. In addition to mass dog vaccination activities to control the disease, public awareness campaign on rabies transmission and prevention for children are employed to increase the knowledge and awareness of school age children on rabies. Since June 2014 to May 2016, a total of 176 schools were visited and 24,325 students had participated in this program. To see the effect of program, a pre- and post-test was conducted, and resulted in a 75.8\% increase of knowledge in participated children. Participated children also showed higher awareness on animal welfare in dogs and responsible pet ownership. It's concluded that rabies awareness campaign for children is vital and can contribute to a reduction of rabies cases in both adult and children in the long term. 


\title{
Ecology and Health: What Drives Disease
}

\section{Gerardo Suzan \\ Universidad Nacional Autonoma de Mexico}

Through a synthesis of current knowledge on disease ecology, the dynamics of pathogens, species diversity and habitat fragmentation will be explored. Given the high variability in different disease systems (e.g. Hantavirus, Lyme disease), longterm monitoring programs have the potential to improve understanding of disease trends at the human-animal-environment interface. Using examples from research in Latin America, surveillance approaches that could be upscaled for greater understanding will be highlighted, as well as potential policy applications of findings for the conservation, agriculture, land use planning and public health communities.

\section{4}

\section{\#JustClimate - A Multi-media Project}

\author{
Melissa Sweet ${ }^{1}$, Summer May Finlay ${ }^{1}$, Kerry Arabena ${ }^{2}$, Marie McInerney ${ }^{1}$ and Mitchell Ward \\ ${ }^{1}$ Croakey.org \\ ${ }^{2}$ University of Melbourne
}

This multi-media journalism project, \#JustClimate, would profile the contributions of Aboriginal and Torres Strait Islander peoples and knowledges to addressing climate change and sustainability. Our team would attend the conference to produce a series of articles, video clips, podcasts, short films, Twitter essays and other digital communications featuring presenters and presentations. It would be a participatory project, seeking to engage other delegates through a multi-media workshop running throughout the conference. All of these works will be packaged into a freely available \#JustClimate e-publication that will be finalised within six weeks of the event. Some of the works will also be published at the social journalism project for health, Croakey.org. This project can be seen as a continuum of Croakey's work at EcoHealth 2013 and engagement with the Oceania EcoHealth Chapter (http://croakey.org/category/croakey-news-and-projects/croakey-conference-news-service/ oceania-ecohealth-symposium-2013/). We acknowledge the Oak Foundation in the US for their grant towards \#JustClimate. This project is informed by a decolonising methodology for journalism practice, and represents the practice of social journalism as a creative form of both knowledge creation and knowledge dissemination.

\section{Leishmania Gap Analysis Study; Lessons for Vector Borne Disease Control in Pakistan}

\section{Qurat Syeda}

District Health Directorate

Given the high prevalence rate of Leishmania in Pakistan, Pak One Health Alliance (NGO) in partnership with international partners conducted Leishmania Disease Gap Analysis Study during April-Nov. 2015. Objectives of this exercise were threefold (i) First to study the existing situation of Leishmania prevention and control at all administrative levels (ii) An evaluation of opportunities and effectiveness of interventions and (iii) Developing set of recommendations for sustainable measures, capacity building with future action plan. Towards this end clinical case data was collated and analyzed to enable endemic zones and calculation of incidence rates. This was compared with vector distribution and prevalence rates in reservoirs. WHO international protocols were used for this assessment. This study made some startling revelations which 
were found useful not only to address menace of Leishmania Disease, but once implemented, could lay the basis of an effective integrated vector borne disease control program in the country.

\title{
Strengthening One Health Capacity in China as Part of Veterinary and Medical Field Epidemiology Training
}

Hao Tang ${ }^{1}$, Fusheng Guo ${ }^{1}$, John Edwards ${ }^{2}$, Baoxu Huang ${ }^{3}$, Youming Wang ${ }^{3}$, Guoqing Shi ${ }^{4}$, Dirk Pfeiffer ${ }^{5}$ and Julian Drewe ${ }^{5}$

${ }^{1} F A O$

${ }^{2}$ Murdoch University

${ }^{3}$ CAHEC

${ }^{4}$ China $C D C$

${ }^{5} R V C, U K$

Two Field Epidemiology Training Programmes for veterinary and medical professionals (CFETPV and CFETP) aim to assist the Chinese government in training epidemiologists in support of zoonotic disease control and prevention in animals and humans. While both programmes are run separately, each has a curriculum with a strong One Health $(\mathrm{OH})$ dimension. In addition, joint training sessions are conducted regularly involving trainees from both programmes. These include an introductory course on $\mathrm{OH}$ and joint workshops on the zoonotic diseases epidemiology and control. During both training programmes, professionals from both sectors are involved are involved in discussing and exchanging ideas, and collaborating in field work whenever possible, based on a common understanding of $\mathrm{OH}$ concepts and approaches gained from the trainings. Through 'learning by doing', the two programs also facilitate and synergize resources for joint field investigation and intervention for trainees and graduates. As a result, cross-sector cooperation will become more effective, also facilitated by the professional and personal relationships established during the training programme. These joint approaches are necessary for dealing with the continued emergence of high impact zoonotic diseases that have national, regional and global impact.

\section{Ebola Economic Impact Study: A Systems Analysis of Preventative Measures Taken by a Mining Firm During the 2014-15 Ebola Outbreak in Liberia}

\author{
Hisham Tariq ${ }^{1}$ and Zia Sadique ${ }^{2}$ \\ ${ }^{1}$ Centre of Disaster Resilience \\ ${ }^{2}$ LSHTM
}

The 'Ebola Economic Impact' study uses a mixed method approach to document the impacts of the 2014-15 Ebola Virus Disease outbreak on the operations of a mining firm in Liberia and estimate the costs of the preventative measures taken by the firm. The study focuses on a single large extractive firm in Liberia and proposes a framework for assessing the operational resilience of the firm. A total of 20 in-depth interviews and 2 focus group discussions were conducted to identify what key employees understood as the impacts on the firm and its operations. The qualitative data indicated the most critical aspects of operations that were impacted in terms of operations, supply chain impacts and the expansion plans for additional mining capacity as well as impacts on daily activities due to the additional preventative measures. The interview data was used to determine the historical context to the financial data that was collected from key departments of the firm. The economic costs of the preventive measures as incurred by the firm were estimated to be between $\$ 10.58-11.11$ 
million. The magnitude of actual costs incurred by the firm largely conforms to the perceived costs impacts identified by the respondents in the qualitative study.

\title{
Scaling-up Intermittent Rice Irrigation for Malaria Control in Peru: The Role of Farmers' Perception and Social Networks
}

\author{
Renata Távora ${ }^{1}$, Frédéric Mertens ${ }^{1}$, Anita Luján ${ }^{2}$, Alain Santandreu ${ }^{2}$, Ernesto Ráez ${ }^{3}$, Elena Ogusuku ${ }^{4}$ \\ ${ }^{1}$ Centro de Desenvolvimento Sustentável-Universidade de Brasília (CDS-UnB)/Community of Practice in Ecosystem Approaches \\ to Health in Latin America and the Caribbean (CoPEH-LAC), Brasilia/DF, Brazil \\ ${ }^{2}$ Consorcio por la Salud, Ambiente y Desarrollo (ECOSAD)/CoPEH-LAC, Lima, Peru \\ ${ }^{3}$ Universidad Peruana Cayetano Heredia, Lima, Peru \\ ${ }^{4}$ Ministry of Health, Lima, Peru
}

Since the 1970s, large-scale irrigation systems in Peru's North coast have enabled a huge expansion of rice cultivation. Irrigation also brought malaria to the region. A pilot ecohealth research showed that Intermittent Rice Irrigation (IRI) could achieve a $90 \%$ mosquitoes decrease in rice paddies, but also higher rice yields and a 30-60\% reduction in water use. The research aimed at characterizing farmers' perception on the social, health, environmental, and economic advantages and trade-offs associated with the adoption of IRI and to understand the role of information exchange networks in the scaling-up of the irrigation technique at the watershed level. Semi-structured interviews were conducted in April 2016, with 368 farmers, 311 men and 57 women, distributed throughout the Jequetepeque watershed. Data included farmers' characteristics, interpersonal communication on agriculture and health, and perceptions of advantages and trade-offs of adopting IRI. Preliminary analyses showed that men value the economic advantages of IRI, while women establish linkages between the new practices and their health benefits. Information on IRI circulates through two distinct gender-specific diffusion pathways. Furthermore adoption for men is associated with participation in communication networks on agriculture, while discussing health is linked to women's adoption. Implications for scaling-up ecohealth interventions are discussed.

576

\section{The Zoonotic Risks Presented by Novel Australian Swine Influenza A Viruses: Impacts at the One Health Interface}

Joanne Taylor ${ }^{1}$, David W Williams ${ }^{2}$, John Bingham ${ }^{2}$, Jeff Butler ${ }^{2}$, Songhua Shan ${ }^{2}$, Daniel Layton ${ }^{2}$, Matthew Bruce $^{2}$, Vittoria Stevens ${ }^{2}$, Dayna Johnson ${ }^{2}$, Hannah Bender ${ }^{2}$, Joanne Meers ${ }^{3}$ and Frank Wong ${ }^{2}$

${ }^{1}$ CSIRO Australian Animal Health Laboratory, University of Queensland School of Veterinary Science

${ }^{2}$ CSIRO Australian Animal Health Laboratory

${ }^{3}$ University of Queensland School of Veterinary Science

Bidirectional spillover transmission of influenza A viruses (IAV) occurs worldwide between human and domestic swine populations. Introduction of human seasonal IAVs into domestic pigs contributes to the diversity of swine influenza viruses due to the propensity for IAV genes to reassort in pigs. IAV strains tend to subclinically circulate in swine herds, undergoing minimal change for decades, and thus pose an ongoing pandemic risk to the human population that may be immunologically naive to swine strains of virus. Following the 2009 emergence of the reassortant pandemic H1N1 (pH1N1) in people, together with the potential for reassortment of avian, human and swine influenza viruses in pigs, there has been an urgent call for increased global surveillance of IAV strains in pigs. In Australia in 2012, reassortant strains of H3N2 and $\mathrm{H} 1 \mathrm{~N} 2$ with $\mathrm{pH} 1 \mathrm{~N} 1$ genes caused respiratory outbreaks in Australian piggeries, indicating the likely endemic presence of 
novel swine IAV in Australian domestic pig populations. The One Health impacts of potential zoonotic transmission events involving these emergent reassortant swine influenza viruses, an assessment of the potential for new pandemic risk, and the need for better understanding and monitoring of IAVs circulating in domestic pig populations in Australia and globally are discussed.

\title{
Assessing Indigenous Food Insecurity: Psychometric Validation of a Culturally-informed Measure Among Pregnant Inuit Women
}

\author{
Lisa Teh ${ }^{1}$, Catherine Pirkle ${ }^{1}$, Chris Furgal ${ }^{2}$, Myriam Fillion $^{3}$ and Michel Lucas ${ }^{3}$ \\ ${ }^{1}$ University of Hawai'i \\ ${ }^{2}$ Trent University \\ ${ }^{3}$ Laval University
}

Globally, food insecurity is a major public health concern, particularly among Indigenous communities. Most food security questionnaires focus on purchased food. However, many Indigenous and remote communities consume a diet that combines foods from the store and traditional foods obtained from the local environment. This study describes the psychometric evaluation of a modified Household Food Insecurity Access Scale (HFIAS), which was developed for mixed economies, to assess food insecurity among pregnant Inuit participants. A modified HFIAS was administered to 131 pregnant women in Nunavik, Canada. Data were fit to a Rasch Rating Scale Model (RSM) to estimate the construct validity. The RSM was a good fit for the data (Infit MSV = .667 to 1.313); however, items primarily assessed moderate levels of food insecurity. A scoring system was developed based on the RSM to improve the scale's accuracy. Addressing food insecurity among Indigenous people requires rigorous, culturally adapted instruments that capture availability, access, quality and utilization of market and traditional food sources. The modified HFIAS shows potential for measuring Indigenous food insecurity. Future studies should integrate specific items about traditional foods. Accurate, culturally appropriate measurements are necessary to understand and support the sustainability of Indigenous food systems and environments.

\section{1}

\section{Impact of Flood on Waterborne Bacterial Pathogens. A Study in Anambra River: South East, Nigeria}

\section{Tehetna Alemayhu Teklewolde}

Mekelle University

Under conditions of climate change, heavy rain and flood would increase the risk of fecal contamination of surface and ground water which is used as drinking water. A cross sectional and retrospective study was carried out to assess the impact of flood on waterborne bacterial pathogens in Anambra River. Bacterial load and isolation of E. coli and Salmonella were performed following the standard microbiological technique. Questionnaire survey of residents of the communities of Otoucha regarding the periods of flood of Anambra River showed that Jun/July marks rising of flood; Aug/Sept peak; Oct/Nov receding and Dec/Jan back to normal water level. There were a significant difference in the mean Total Viable Bacterial Count (TVBC) (cfu/ml) and mean Coliform Counts (CC) between Aug/Sept and Dec/Jan at point of water collection for household purposes and for washing of meat at slaughter slab. Furthermore, the mean TVBC and CC of meat after washing were significantly higher than before washing. Verotoxigenic E. coli and Salmonella were isolated from the flood water and meat. As to the retrospective study, the result revealed that out of a total of 433 confirmed diarrheic cases registered in two hospitals, 228 (52.66\%) were registered in 2012 when Nigeria experienced the worst flooding, including the Anambra River. 


\title{
YIMBY! Yes in My Back Yard
}

Teresa Teresa Capetola, Claire Henderson-Wilson, Justin Lawson and Berni Murphy School of Health and Social Development Deakin University

An often familiar cry by sustainability opponents is Not In My Back Yard (NIMBY), protesting that sustainability action threatens current lifestyles or landscapes. Between 2014-2016 the Health Nature and Sustainability Research Group, Deakin University, Melbourne, undertook a deliberative program to both profile and model sustainability action within the University's largest campus through two research programs focused on food. This action speaks directly to the social and cultural determinants of health as a means of simultaneously influencing these determinants for positive health and imbuing them with sustainability content. Firstly a feasibility study returned over 500 positive responses to the establishment of a campus based community garden. Critical mass lead to the formation of a student sustainable food society. Following two years of campaigning, the university approved a campus based community garden in early 2016. A second research investigated the "food culture" promulgated across the campus through a survey of staff, student and food outlets and an audit of facilities for food preparation, storage and disposal. Findings revealed hidden food insecurity, dissatisfaction with campus based food options and an ineffective recycling of food waste. Yes In My Back Yard (YIMBY) actions to positively address these concerns and influence the social and cultural determinants of health are being pursued.

\section{5}

\section{Global Dynamics of Highly Pathogenic Avian Influenza Viruses Between 2005 and 2015: Spread and Speed Analysis}

\author{
Paolo Tizzani, Lina Awada, Neo Mapitse and Paula Caceres \\ World Organisation for Animal Health
}

Highly Pathogenic Avian Influenza (HPAI) was the most often reported disease in terms of number of alerts submitted by countries to the World Organisation for Animal Health (OIE) between 2005 and 2015. Knowledge of HPAI viruses' dynamics is important in the prevention of their further spread. To perform such analysis, the OIE World Animal Health Information System (WAHIS) is the global reference source for confirmed information. The aim of the paper is to describe the global dynamics of HPAI viruses over the last 11 years, their capability in terms of spread and speed in order to enhance the capacity of predicting and managing epidemics. An increase in the number of affected countries and the circulating virus subtypes was observed in recent years. The analysis considered 6,546 outbreaks belonging to 289 events (epidemiologically related outbreaks) in 73 countries between 2005 and 2015. The median spread value from the index outbreak was $131 \mathrm{~km}$ and the median speed value was $2.4 \mathrm{~km} /$ day. Significantly higher median values were registered when wild birds were involved: $223 \mathrm{vs} 131 \mathrm{~km}$ for spread and 11.7 vs $1.7 \mathrm{~km} /$ day for speed. Spread and speed results variations over time were described and discussed.

\section{7}

\section{Combining Experts, Government and NGO's to Sustainably Eliminate Disease}

\section{Lien Trinh \\ Rotary Club of Melbourne}

A major problem in disease eradication programs is ensuring that the interests and objectives of all potential partners are combined and harnessed to efficiently achieve the desired outcomes. Following on from its successful participation in the 
global eradication of polio, Rotary in Australia is participating in the WHO Global Elimination of Trachoma by 2020 project. Despite substantial Government funding for this cause to date, Australia remains the only developed country where trachoma is still endemic. Rotary has established partnerships with subject experts, institutions, health authorities and NGO's, to enhance their expertise and delivery approaches with an objective overview. While subject experts and research institutions deliver the medical interventions required to eliminate blinding trachoma, Rotary enhances the practical action and activities related to hygiene, environment and education that are essential to targeting the root cause of disease and preventing the spread of infection. Through Rotary's extensive network of community leaders, professionals, businesses and with organisations such as Plan International and Soapaid, we are targeting the public health interventions that will end trachoma completely, significantly reduce the incidence of concurrent diseases, and lead to the alleviation of poverty among our least advantaged Australians.

\title{
1098
}

\section{One Health - A Hong Kong Perspective}

\author{
Cherry Tse Ling Kit-ching JP \\ Permanent Secretary for Food and Health
}

Hong Kong is no stranger to new and emerging infectious diseases, with the first case of humans infected with H5N1 avian influenza (AI) and severe acute respiratory syndrome (SARS) reported respectively in 1997 and 2003. As our awareness of the vulnerability of human health to practices affecting animal and environmental wholesomeness grows, so must we revisit our approach to managing risks. Discrete risk management must give way to an integrated approach. The "One Health" concept has at its core the recognition of the symbiotic relationships among humans, animals and the world in which we dwell. Like other places, Hong Kong embraces a "One Health" approach to tackling zoonotic diseases and safeguarding public health. The last two decades have seen the increasing integration of veterinary with public health expertise in policy making and in tackling food incidents and threats of infectious diseases. Hong Kong is one of the few jurisdictions where the oversight for both animal and human health is put under one policy roof. Over the years, there have been occasional AI and food safety incidents. We follow up each with a rigorous review of our strategy and operational procedures. No review is complete without the involvement of both veterinary and public health expertise. Looking back, we have come a long way. Still, our system is far from perfect. Safeguarding humans and animals from zoonotic diseases and reaching the lofty food safety target of "from farm to table" is an uphill task. The real world comprises more than just veterinary and public health personnel. Nor is the veterinary and public health sector an island-its proper functioning relies on many other players. As we globalize exchanges of all sorts, threats to human and animal health also transcend jurisdictional boundary. International collaboration is not only desirable, it is essential. These put the "One Health" concept in an even broader perspective. The concomitant diversity of perceptions, interests, values and cultures is what we must grapple with. With illustrations drawn from our experience in handling a number of food safety and infectious disease incidents, Hong Kong would like to share our reflections on the practice of "One Health" that must encompass, but also go well beyond, our local veterinary and public health sector.

\section{Importance of Parasitic Foodborne Diseases in Rural Areas of Southern Laos - A Long Term Case Study Using an Integrated Approach}

Vannaphone Putthana ${ }^{1}$, Panomsak Promburom ${ }^{2}$, Thipphakone Lacksivy ${ }^{3}$, Dethaloun Meunsene ${ }^{1}$, Amphone Keosengthong ${ }^{1}$, Garance Danner ${ }^{4}$, Khao Keonam ${ }^{1}$, Palamy Changleuxai ${ }^{1}$, Nalita Adsanychanh ${ }^{1}$, Daosavanh Sanamxay ${ }^{1}$, Fongsamouth Southammavong ${ }^{1}$, Stephan Herder Stephan Herder ${ }^{5}$ and Fred Unger ${ }^{6}$

${ }^{1} \mathrm{NUoL}$

${ }^{2} \mathrm{CMU}$ 
${ }^{3} K U$

${ }^{4} C I R A D$

${ }^{5} I R D$

${ }^{6} I L R I$

Parasites foodborne diseases (PFBD) are expected to be widely distributed in Laos and can have a significant impact on health but also on economy and livelihood. Detailed information on their distribution is lacking and risky food consumption habits exist (e.g. consumption of raw or rare meat/fish). The objectives of this ongoing study are to assess PFBD distribution and risk-related practices in 3 provinces of southern Laos (Savannakhet, Khammuane and Champasak), and establish a cross-sectorial collaboration platform which aims to develop and promote feasible control options addressing needs of affected communities and stakeholders. To facilitate and monitor community involvement and cross-sectoral collaboration among stakeholders integrated approaches such as companion modelling (ComMod) and One-Health are used synergistically. A multidisciplinary research team consisting of vets, public health, environmental, social and participatory modelling scientists has been established and jointly implemented activities using quantitative (e.g. serological sampling) and qualitative methods such as PRA and Participatory Epidemiology. Villagers had lowest PFBD knowledge while para-meds and teachers had highest. Flood or lack of irrigation were ranked highest by villagers while PFBD were never considered as important issues. Serological data on Trichinelloses and Cysticercoses in pigs are currently analysed. Policy engagement and feedback is facilitated through quarterly meetings.

452

\section{Food Safety Along Informal Pork Value Chains In Vietnam - Achievements and Challenges from an Interdisciplinary Research Team View}

Fred Unger ${ }^{1}$, Hung Nguyen-Viet ${ }^{1}$, Steven Lam $^{2}$, Lucy Lapar ${ }^{1}$, Phuc Pham Duc ${ }^{2}$, Hung Pham Van ${ }^{3}$, Ngan Pham Hong $^{3}$, Max Barot ${ }^{1}$, Sinh Dang-Xuan and Delia Grace ${ }^{1}$

${ }^{1} I L R I$

${ }^{2}$ HSPH/CENPHER

${ }^{3}$ VNUA

Pork is widely consumed meat in Vietnam, making up 56\% of total meat intake. While delivering significant benefits to smallholders who supply $80 \%$ of the market, pork could also cause substantial health consequences due to poor hygiene along the pork chain. To identify feasible interventions along the pork chain, a better understanding of disease risks is needed and addressed in an ongoing research project (PigRISK) presented here. PigRISK uses an interdisciplinary research approach, bringing together livestock economic, animal health, risk assessors, environmental and public health expertise targeting smallholder pig value chains in Hung Yen and Nghe An provinces, Vietnam. Selected technical achievements include value chain maps, hazard estimates ( $>40 \%$ of pork Salmonella contaminated) and health risks which will be presented. From an interdisciplinary research perspective and documented using outcome harvesting/mapping, PigRISK builds capacity on assessing health risks, value chains and also enhances collaboration among teams. This was achieved through providing the opportunity for researchers to work "day to day" across disciplines and institutions, a new experience for most of them. Challenges include high turnover of team members or analysis and synthesis of results outside the team member's common expertise. Interventions, currently developed, will be piloted in a second project phase. 


\section{Assessing the Impact of Treating Commercial Piglets with a 3rd Generation Cephalosporin Injection on Cefotaxime Resistance of Commensal Faecal}

E. Coli

Ana Vale ${ }^{1}$, James Gibbons ${ }^{1}$, Edgar Manzanilla ${ }^{2}$, John Egan ${ }^{3}$, Seamus Fanning ${ }^{4}$, Laura Boyle ${ }^{2}$ and Finola Leonard ${ }^{1}$ ${ }^{1}$ School of Veterinary Medicine University College Dublin

${ }^{2}$ Teagasc Pig Production Development Unit

${ }^{3}$ Central Veterinary Research Laboratory DAFM

${ }^{4} U C D$ School of Public Health, Physiotherapy \& Population Science

Antimicrobial resistance (AMR) poses a complex threat to the human-animal-environment interface. Global use of antimicrobials (AMs) in human medicine, veterinary medicine and plant agriculture compromises antimicrobial efficacy. To safeguard the correct use of antimicrobials WHO and OIE have created lists of antimicrobial classes according to their importance in treating infections: third-generation cephalosporins (TGCs) are included in both lists as critically important AMs.

In Irish pig production TGCs are mainly used in piglets to treat infections such as meningitis. Faecal samples from 38 three-days old piglets (12 untreated piglets in control pens, 14 treated and 12 untreated piglets in treatment pens) from a commercial farm with no previous use of TGCs were investigated to evaluate the impact of a long-acting TGC injection (Ceftiofur) on cefotaxime resistance in commensal E. coli. Faecal swabs were collected at 7 time-points over a 22-day period from piglets, their dams (8 sows) and environment. Samples were screened for the presence of cefotaxime-resistant E. coli using TBX agar supplemented with cefotaxime according to EUCAST guidelines.

All $E$. coli isolates screened during the trial were cefotaxime-susceptible suggesting that single use of TGC on a naïve farm does not increase the number of cefotaxime-resistant E. coli detected.

\section{Important Resistance Genes Carried in Non-pathogenic Escherichia coli in Australian Piglets}

Lechelle van Breda $^{1}$, A. N. Ginn ${ }^{2,3}$, O. P. Dhungyel ${ }^{1}$, S. R. Partridge ${ }^{3}$, J. R. Iredell ${ }^{3}$ and M. P. Ward

${ }^{1}$ The University of Sydney, Faculty of Veterinary Science, Camden, NSW 2570 Australia

${ }^{2}$ Antimicrobial Resistance Reference Laboratory, Centre for Infectious Diseases and Microbiology Laboratory Services, Pathology West, Westmead, NSW 2145, Australia

${ }^{3}$ Centre for Infectious Diseases and Microbiology, The Westmead Institute for Medical Research, The University of Sydney, Westmead Hospital, Westmead, NSW 2145, Australia

The Australian pig industry often uses antibiotics at weaning for treatment of diarrhoea in piglets caused by Escherichia coli. Our aim was to isolate E. coli from healthy and sick piglets to determine resistance to third generation cephalosporin antibiotics (3GC) used in veterinary medicine (e.g. ceftiofur). A survey was conducted in 22 commercial piggeries in South Eastern Australia (New South Wales n = 9; Victoria $n=10$; and South Australia n = 3) from September 2013 to May 2014. From each herd, 10 pre- and 40 post-weaned piglet faceal samples were spread onto Sheep blood agar and CHROMagar Orientation to isolate E. coli. A total of 325 E. coli isolates (15 from each herd) were tested for resistance to 17 veterinary antibiotics (CLSI and EUCAST guidelines) and enterotoxigenic (ETEC) genes (F4, F5, F6, F18, F41 and STa, STb, LT) commonly associated with piglet diarrhea. Phenotypic resistance to $3 \mathrm{GC}$ e.g. ceftiofur $(\geq 8 \mu \mathrm{g} / \mathrm{mL})$ was identifed in $5 \%$ (17/325). Of the 17 resistant isolates 14 lacked ETEC genes suggesting that $3 \mathrm{GC}$ resistance is circulating at higher pro- 
portions within non-pathogenic E. coli isolates at the farm level. Further analysis of 3GC resistance patterns in finisher pigs is essential, due to their relevance to potential zoonotic transmission.

Keywords: Antibiotic resistance; Escherichia coli; third-generation cephalosporin; piglets; Australia

195

\title{
A One Health Approach to Investigation of Zoonotic Hepatitis E Virus in Cape Town, South Africa
}

Lesley van Helden ${ }^{1}$, Stephen Korsman ${ }^{2}$, John Grewar ${ }^{3}$, Peter Thompson ${ }^{4}$, Saarah Isaacs ${ }^{2}$, Kim Picozzi ${ }^{5}$, Monique Andersson ${ }^{6}$ and Wolfgang Preiser ${ }^{6}$

${ }^{1}$ Western Cape Veterinary Services

${ }^{2}$ National Health Laboratory Service

${ }^{3}$ University of Witwatersrand

${ }^{4}$ University of Pretoria

${ }^{5}$ University of Edinburgh

${ }^{6}$ University of Stellenbosch

Genotype 3 of hepatitis E virus (HEV) was recently isolated from three patients with hepatitis in Cape Town, South Africa. As pigs are the reservoir of HEV genotype 3, prevalence of HEV was investigated in a cross-sectional study of 16 commercial pig herds supplying pork to Cape Town. A strain of HEV genotype 3e related to the human strains from Cape Town was identified in serum of one of the sampled pigs. A high HEV seroprevalence was found, with a median within-herd prevalence of 0.93 . Preliminary analysis of factors on farms from which sampled pigs had originated identified several factors which were associated with HEV seropositivity at slaughter, including age-group mixing, increased contact between pigs and manure, inadequate pen resting times, and lack of general biosecurity measures. This is the first study to investigate the presence and epidemiology of HEV in South African pigs. The findings indicate that strategies to prevent cases of the disease can only be approached from a one-health perspective to include prevention of transmission between pigs, prevention of zoonotic spread to animal workers and pork consumers, and adequate control of farm and abattoir waste to prevent contamination of the environment.

\section{Practical Experiences with a One Health Approach in Dairy Farming: International Collaboration Towards Responsible Antibiotic Use and Improved Milk Quality}

\author{
Katrien van't Hooft ${ }^{1}$, M.N.B. Nair ${ }^{2}$, Getachew Gebru' ${ }^{3}$, Elizabeth Katushabe ${ }^{4}$ and Maria Groot ${ }^{5}$ \\ ${ }^{1}$ Dutch Farm Experience \\ ${ }^{2}$ Trans Disciplinary University (TDU) \\ ${ }^{3} E S A P$ \\ ${ }^{4}$ COPACSO \\ ${ }^{5}$ RIKILT
}

Dairy farming is regarded as one of the ways to satisfy the growing demand for high- quality animal protein worldwide. Growing intensification of dairy production systems has also lead to increased use of antibiotics and other chemicals. This negatively affects food quality and the environment, while AMR (Anti Microbial Resistance) is rapidly becoming one of the main challenges for human and animal health. Since 2014 Dutch Farm Experience is collaborating with international partners on improving milk quality and responsible use of antibiotics in dairy farming. Organized as the international 
Natural Livestock Farming (NLF) network farmers, veterinarians and other experts are developing practical solutions, combining the expertise from four countries: India, the Netherlands, Ethiopia and Uganda. After two years of successful exchange programs the so-called NLF five-layered approach was developed: improved animal health practices, strategic use of local breeds, herbal medicine, milk quality control and extra payment to farmers for residue-free milk. Based on a One Health Approach a joint initiative was formulated for the coming years: 'Healthy Cows-Healthy Food-Healthy Environment: Developing the One-Health approach to reduce the use of antibiotics and other chemicals in dairy farming through the implementation of the Natural Livestock Farming five-layered strategy'

\title{
Defining the Epidemiology of Zoonotic Neurological Arboviruses Through a One Health Approach in South Africa, 2008-2016
}

\author{
Marietjie Venter ${ }^{1}$, Marthi Pretorius ${ }^{2}$, Voula Stivaktas ${ }^{2}$, Jumari Steyn ${ }^{2}$, Mpho Rakganto $^{2}$, James Fuller ${ }^{3}$ and June \\ Williams ${ }^{2}$ \\ ${ }^{1}$ Department Medical Virology University of Pretoria \\ ${ }^{2}$ University of Pretoria \\ ${ }^{3}$ Global disease Detection, US-CDC, South Africa
}

We investigate the epidemiology of neurological zoonotic arboviruses in South Africa, through passive surveillance between 2008-2016 for acute febrile and neurological disease in horses, livestock and wildlife. Blood or neurological tissue for fatal cases were screened for alpha-, flavi-, bunya and encephalosis virus (EEV) using polymerase chain reaction plus West Nile Virus (WNV) IgM ELISA and neutralization assays in horses. In total, 274/1654 (22\%) cases tested positive: 249 (20\%) horses, 6 (4.3\%) livestock and 19 (7\%) wildlife. Among horses, WNV was most frequently identified ( $\mathrm{n}=96 ; 38.5 \% \% ; 34 \%$ fatal) followed by $\operatorname{EEV})(\mathrm{n}=73 ; 5.9 \% ; 13.7 \%$ fatal $)$ Middelburg-(MIDV) $(\mathrm{n}=77 ; 29.3 \% ; 33.8 \%$ fatal), Shuni(SHUV) $(\mathrm{n}=23 ; 23.9 \% ; 52.2 \%$ fatal), Sindbis- $(\mathrm{n}=23 ; 23.9 \% ; 10 \%$ fatal $)$ and Wesselsbronvirus $(\mathrm{n}=2 ; 2 \% ; 50 \%$ fatal $)$. Of 19 positive wildlife cases, $15(78.9 \%)$ were fatal. Wildlife tested positive for Middelburg $(n=10 ; 52.6 \%, 80 \%$ fatal); SHUV $(\mathrm{n}=4 ; 21 \%, 100 \%$ fatal $)$, Sindbis $(\mathrm{n}=5 ; 26.3 \% ; 100 \%$ fatal $)$ and EEV $(\mathrm{n}=1 ; 5.2 \% ; 100 \%$ fatal $)$. Positive species included buffalo, rhinoceros, sable, giraffe, warthogs, crocodiles, lion and genet. Of 141 livestock tested 5 Middelburg ( $80 \%$ fatal) and 2 WNV $(100 \%$ fatal) cases were identified in cattle. Cases occurred primarily from February-June, peaking in March throughout the country following peak rainfall. Geographic location of veterinarians seropositive for WNV and SHUV identified in a serosurvey in 2010-2011 correlated with animal cases suggesting these arboviruses are widespread in the country and should be considered in animal and human neurological disease.

683

\section{Extractive Industries Role in One Health}

Francesca Viliani

The extractive sector is an important player within the one health system. Firstly, the extractive sector plays an important role in the economy of many low-and-middle income countries, many of which lie in hotspots for emerging and re-emerging infectious diseases. Secondly, the sector has a strong health and safety culture that lead companies to incorporate infectious disease control programmes as part of their in-country projects. Thirdly, activities related to mega project development and wider natural resource extraction play a key role in altering the factors and risks associated with disease emergence. Land-use, human movement and biodiversity are all fundamentally altered as a direct consequence of initiating extractive projects. The Ebola outbreak in West Africa has been a game changer for the role played by the private sector, including extractive companies, in the management of outbreaks and possibly other health emergencies. The presentation 
will examine how a one health framework might improve EID management in the extractive sector: starting at the project conceptualization and impact phase; developing a robust surveillance and monitoring system for possible impacts, and establishing partnerships to increase resilience for the project and the communities.

962

\title{
Hospital Sentinel Surveillance for MERS-CoV, Zika Virus, and Yet-to-be- Detected Pathogens Through a One-Health Lens
}

\author{
Supaporn Wacharapluesadee ${ }^{1}$, Kevin $\mathrm{J} \mathrm{Olival}^{2}$ and Thiravat Hemachudha \\ ${ }^{1}$ WHO Collaborating Centre for Research and Training on Viral Zoonoses, Thai Red Cross, Faculty of Medicine, Chulalongkorn \\ University \\ ${ }^{2}$ EcoHealth Alliance, New York
}

Developing countries are at risk for outbreaks of emerging infectious diseases due to limited preparedness given economic resources and diagnostic capacity. Targeting certain hospitals for sentinel surveillance allows for earlier detection of diseases. In Thailand, detection of novel and known viruses has been implemented in Chulalongkorn Hospital laboratory (CHL), the country's reference PREDICT laboratory. The experienced well-qualified staffs at CHL have been trained to handle and characterize novel viruses including coronaviruses, flaviviruses and filoviruses in both wildlife and human samples using PREDICT protocol. Recently, two imported MERS cases in Thailand were first diagnosed and confirmed here, within $24 \mathrm{~h}$. No secondary infection followed these cases, and more than 100 close-contacts from each case were tested. In 2016, Zika virus was detected in 9 provinces through active surveillance carried out by the Thai government, tested at CHL. Human transmission was controlled within 1-2 months in all provinces, as a result of early laboratory detection and rapid reporting. The high-quality diagnostic data, as well as the One-Health collaborative approach between hospitals, CHL, Ministry of Public Health, Department of National Parks, and Department of Livestock Development has been key for the success of controlling disease transmission in the country.

\section{6}

\section{Geo-Surveillance of Viral Hemorrhagic Fevers in Cameroon: Case study of Filoviruses in Bats}

\author{
Abel Wade ${ }^{1}$, Rodrigue N Poueme ${ }^{2}$, Abdoulkadiri Souley ${ }^{2}$, Martin Grushop, Mbacham F Wilfred ${ }^{3}$, Michel \\ Warnau ${ }^{3}$ and Hermann Unger ${ }^{4}$ \\ ${ }^{1}$ Laboratoire National Veterinaire (LANAVET) Annexe Yaoundé \\ ${ }^{2}$ LANAVET Garoua \\ ${ }^{3}$ Biotechnology Centre, University of Yaounde I \\ ${ }^{4}$ Friedrich Loeffler Institute (FLI)
}

Viral hemorrhagic fevers (VHFs) refer to diseases caused by several groups of viruses which include zoonotic potentials like Ebola and Marburg. Routes of their transmission are variable, and migratory fruit bats are known to be carrier reservoirs. We induced a geo-monitoring system of these bats and evaluated biorisk factors in Cameroon and across international borders. Blood and swap samples were also collected for molecular screening of filoviruses. Our findings indicate that bats in Cameroon are illegally hunted for human consumption with high biorisk of viral spread to human. Age estimate was based on wing measurements. Global Positioning System (GPS) chips were fixed on the neck of some colony-distributionbased bats for geo-monitoring at the national and international transboundary levels using computer software system. This geo-surveillance allowed monitoring of bats movement in the countries with some colonies crossing international borders 
to Nigeria and Chad. Laboratory screening of 298 sampled bats using 'RealStar Filovirus Screen RT-PCR Kit' indicate total absence of Ebola and Marburg viruses in Cameroon. This eating habit should be corrected to reduce the biorisk. Such study should be conducted yearly in many countries worldwide, taking into account all VHF that can be transmitted by through bats.

162

\section{Bats and Viruses: A Rich Source Of One Health Lessons}

\section{Linfa Wang}

Programme in Emerging Infectious Diseases, Duke-NUS Medical School

Approximately $75 \%$ of emerging infectious diseases are zoonoses. The rate of emergence of zoonotic viruses appears to be increasing and/or our ability to detect new viruses is improving. Bats are being increasingly recognised as an important reservoir of zoonotic viruses of different families, including SARS coronavirus, Nipah virus, Hendra virus and Ebola virus. Several recent studies hypothesized that bats, an ancient group of flying mammals, are the major reservoir of several important RNA virus families from which most (if not all) other known mammalian viruses of livestock animals and human were derived. Although this hypothesis needs further proof, the fact that bats carry a large number of viruses is commonly accepted. The question of whether bats have unique biological features making them ideal reservoir hosts has been the focus of research in our research group for the past few years. Our latest findings will be presented to demonstrate that the bat-virus continuum is a rich source for One Health research and there are potentially many lessons we can learn from bats to better respond to future emerging infectious disease events.

\section{Rabies in Northern Australia: Preparedness and Response}

Michael Ward ${ }^{1}$, Victoria Brookes ${ }^{1}$, Salome Dürr ${ }^{2}$, Courtenay Bombara ${ }^{1}$, Emily Hudson ${ }^{1}$, Vanesaa GabrieleRivet $^{1}$, Beth Cookson ${ }^{3}$ and Nick Harris ${ }^{3}$

${ }^{1}$ The University of Sydney

${ }^{2}$ University of Bern

${ }^{3}$ Department of Agriculture \& Water Resources

The risk of exotic disease incursion is an increasing problem, driven by human-mediated transport of animals. Rabies is endemic in Indonesia; Australia's extensive and remote northern borders are difficult to regulate, and a rabies infected (but nonclinical) dog on a fishing vessel or pleasure craft poses a risk. The impact of a rabies incursion on domestic dog and dingo populations could be enormous, and would cause health impacts and social disruption within indigenous communities. Beginning 2012, a research program was initiated to investigate how rabies might spread to northern Australia and its potential impact. It has focused on incursion pathways and risk assessment in PNG, the Torres Strait and Cape York Peninsula. The demographics of free roaming dog populations within indigenous communities on the tip of Cape York Peninsula have been studied intensively, using sight-resight methods, GPS and video collars, genetics and questionnaire surveys. Estimation of the size and distribution of surrounding wild dog and dingo populations has recently commenced, using motion-activated cameras and genetics. To inform plans for response and control, risk assessment and disease spread models are being developed. This decision-support system will facilitate planning for a worst-case scenario-a rabies incursion in remote northern Australia. 


\title{
Environmental Water: The Lifeblood of Aboriginal Health and Wellbeing
}

\author{
Neil Ward and Susan Goff \\ Murray-Darling Basin Authority
}

The assertion that connection to Country underpins Aboriginal wellbeing is at once obvious and contested. Using participatory approaches to socioeconomic and environmental research the Aboriginal Partnerships team at the Murray-Darling Basin Authority (MDBA) are developing tools with Aboriginal Nations to communicate the value of environmental water in terms consistent with Chapter 10 Part 14 of the Basin Plan. There is a direct relationship between environmental water and cultural, natural, physical, financial, social and human assets. But how can this connection be convincingly explained to nonAboriginal decision-makers in the water game? Will arguments about Ancestral rights result in allocations of water substantial enough to redress injustices and drive self-determination? Representatives of the Partnership will present findings from an extensive literature review regarding the dimensions of health and wellbeing that are evident in the many ways in which Aboriginal Nations relate to their Country. Drawing on these insights they will then foreshadow a new five-year inquiry into the social impacts of the ultimate source of environmental water, the weather, on Aboriginal lives in the Basin.

1010

\section{Climate and Health Co-benefits from Changes in Diet. A Case Study from Austria}

Ulli Weisz ${ }^{1}$, Hans-Peter Hutter ${ }^{2}$, Cem Ekmekcioglu ${ }^{2}$, Michael Kundi ${ }^{2}$, Peter Wallner ${ }^{2}$, Christian Lauk ${ }^{1}$, Willi Haas $^{1}$, Jennifer Delcour ${ }^{3}$, Robert Griebler ${ }^{4}$, Peter Nowak $^{4}$, Karl Steininger ${ }^{3}$, Brigitte Wolkinger ${ }^{3}$ and Michaela Theurl $^{5}$

${ }^{1}$ Institute of Social Ecology Vienna, Alpen-Adria University Klagenfurt

${ }^{2}$ Medical University Vienna, Institute for Environmental Health

${ }^{3}$ Wegener Center for Climate and Global Change, University of Graz

${ }^{4}$ Austrian Public Health Institute ( $\left.G O ̈ G G m b H\right)$

${ }^{5}$ Institute of Social Ecology Vienna, Alpen-Adria University Klagenfurt and Research Institute of Organic Agriculture Austria (FIBL)

Diets are crucial for the environment and for health. We examine co-benefits from changes in diet using Austria as case study. As a novel aspect, our analysis covers the entire causal chain from feasibility of implementing a policy via the effectiveness of the measures, their impacts on diets, up to the resulting climate and health benefits. Leverage points for achieving co-benefits are examined with two scenarios and benchmarked against the baseline year. The scenarios focus on reducing meat intake, known to yield strongest effects. The happier animal scenario (HA) introduces recommended animal welfare standards. Additionally, the healthier people scenario (HP) implements a meat tax used to subsidize fruits and vegetables. The results reveal that meat intake can be reduced by $20 \%$ (HA) and $50 \%$ (HP) leading to a $26 \%$ and $60 \%$ reduction of greenhouse gas emissions respectively ( 2 and 5 million tons/year, calculated as 50 years average). The health benefits show strongest effects in overall mortality in the HP-scenario, where 110.000 life years are saved annually. The incidences of colon cancer and diabetes decline by $10 \%$ and $15 \%$ respectively. The study stresses the importance of integrated policies that strive for both protecting the climate and promoting health. 


\section{Metagenomic Studies of Infection Transmission Between Multiple Species in Rural Uganda}

Janelle Wierenga $^{1}$, Kristene Gedye ${ }^{1}$, Gladys Kalema-Zikusoka ${ }^{2}$, Willy Valdivia ${ }^{3}$, Richard Hall ${ }^{4}$, Patrick J. Biggs ${ }^{1}$ and David T.S. Hayman

${ }^{1}$ Massey University

${ }^{2}$ Conservation Through Public Health

${ }^{3}$ Orion Integrated Biosciences, Inc.

${ }^{4} E S R$

Transmission of infections between species can contribute to significant outbreaks of disease in human and animal populations. Factors required for infectious disease transmission to occur between species are usually inferred after serious outbreaks but typically go unnoticed and rely on observable disease and its notification. We investigated populations on the border of Bwindi Impenetrable Forest in southwestern Uganda where endangered mountain gorillas, human and livestock interactions have resulted in transmission of zoonoses and reverse zoonoses, sometimes with serious consequences such as with scabies mortality in critically endangered gorillas. Faecal samples from mountain gorillas, regional cattle and clinicallyunwell humans were analysed by next-generation sequencing to evaluate for viral, bacterial, protozoan and parasitic pathogen presence. We hypothesized that pathogens would be shared between these populations, but that particular host (e.g. relatedness) and pathogen traits (e.g. occupying the gastrointestinal tract niche) and ecological processes (e.g. contact rates) would drive different infection-sharing patterns. Preliminary analyses using k-mer based motif searches revealed similar pathogen signatures in samples from humans, mountain gorillas and cattle. Putative pathogen motifs were either shared between humans and cattle or humans and gorillas, suggesting both contact rates and host phylogeny may be important factors in facilitating pathogen sharing.

445

\section{Clinical One Health - A Medical Perspective}

\section{Kathryn Wilks \\ Queensland Health}

The One health approach includes communication and interrelation between physicians, ecologists and veterinarians to monitor and control public health threats. Public health threats are not just limited to communicable diseases and zoonoses, but also threats to the food chain and safe environment.

The contribution by physicians to the One Health concept may be hampered by a lack of appreciation of the inherent interrelatedness of all species and the environment due to limited undergraduate exposure to these concepts. Undergraduate teaching often focuses on zoonoses, but less on the benefits of animal exposure. Humans are often seen in isolation and distinct from other animals and the environment. The sub-specialization of human medicine also limits the practice of One Health concepts.

One Health can be incorporated into medical practice at a clinical level. Examples of this including the linking of humans brucellosis to canine brucellosis cases with veterinary consultation and the impact of the 2011 Queensland floods on human illness. 


\title{
Epidemic and Endemic Mosquito-borne Flaviviruses of the Asia-Pacific Region: Their Importance in Diseases of Humans and Animals
}

\author{
David Williams ${ }^{1}$ and John Mackenzie ${ }^{2}$ \\ ${ }^{1}$ CSIRO, Australian Animal Health Laboratory, Geelong \\ ${ }^{2}$ Curtin University, Perth
}

There are approximately 20 known species of mosquito-borne flaviviruses that circulate in the Asia-Pacific region, including a number of known pathogens. Normally most of these viruses exist in animal-mosquito transmission cycles. Over the past 20 years, a number of flaviviruses have emerged unexpectedly to cause outbreaks of human and/or animal disease. Japanese encephalitis emerged in northern Australia in 1995, causing human cases of disease; Zika virus emerged in the Western Pacific from SE Asia in 2007 as a significant human pathogen, and has since spread to several Pacific islands as well as to the Americas; and Murray Valley encephalitis re-emerged in south eastern Australia to cause human and equine disease, together with West Nile virus, which caused unprecedented numbers of equine disease. In each of these examples, a confluence of favourable vertebrate host reservoir and environmental factors occurred to allow these viruses to become established, including the presence of immunologically naïve human and/or animal populations and competent vectors. These examples of flavivirus diseases and the factors underlying their emergence or re-emergence will be presented in the context of One Health, and their potential for pandemic spread will be explored.

\section{7}

\section{Promoting the Direct Human Consumption of Mutton and Sheep Offal to Benefit Human Nutrition, Environmental Sustainability and Sheep Welfare in Australia}

\author{
Kate Wingett ${ }^{1}$, Margaret Allman-Farinelli ${ }^{2}$ and Robyn Alders ${ }^{1}$ \\ ${ }^{1}$ Faculty of Veterinary Science, University of Sydney \\ ${ }^{2}$ Faculty of Science, University of Sydney
}

Two major challenges facing the global community are the widespread prevalence of diet related non-communicable disease and climate change. With the world population projected to reach over 9 billion by 2050 these issues will only heighten in the coming decades. The reduction of food loss and waste is one strategy that can assist in meeting these challenges. In 2011, 9,900 tonnes of edible offal produced in Australia were wasted and a further 19,600 tonnes of edible offal and 23,800 tonnes of mutton were used for purposes other than direct human consumption. Greater utilisation of sheep carcases and carcase parts for direct human consumption has the potential to benefit human health and the natural environment. This would be achieved through improving the micronutrient status of populations, in particular iron levels, and by increasing the yields of sheep producers, hence lowering greenhouse gas emission intensities and land clearance rates. This approach supports improved sheep health and greater gross margins in the sheep meat value chain. Quantifying and qualifying the loss of mutton and sheep offal from the human food chain, together with refining the communication pathways in the Australian sheep meat value chain, will contribute to sustainable and healthy domestic food systems. 


\title{
245
}

\section{Australia's Bat Health Focus Group - One Health in Action}

\author{
Keren Cox-Witton, Rupert Woods and Victoria (Tiggy) Grillo \\ Wildlife Health Australia
}

The One Health concept has grown out of the recognition that human health, domestic animal health and wildlife health are strongly interlinked with each other and the environment. Recent disease outbreaks have raised the profile of bats within this paradigm. Wildlife Health Australia (WHA), the national peak body for wildlife health, is an example of One Health in action, coordinating a growing network of over 600 wildlife health professionals around Australia from a range of disciplines. WHA's Bat Health Focus Group is a successful illustration of this approach. Members include representatives from commonwealth and state/territory government agencies dealing with public health, agriculture and environment, university researchers, ecologists, virologists, epidemiologists, veterinarians, wildlife/bat carers, cavers, and the Australasian Bat Society. Using a collaborative One Health approach, the group considers bat health issues in relation to the broader context of public health, biosecurity, livestock health, biodiversity and environmental impacts in Australia. Focus is on endemic zoonotic diseases such as Australian bat lyssavirus and Hendra virus, as well as preparedness for exotic diseases such as rabies and white-nose syndrome. Improved communication and collaboration across sectors allows for a more coordinated approach to investigation and management of wildlife health in Australia.

\section{Wildlife Health Australia and One Health: What's Working and Why?}

\section{Rupert Woods \\ Wildife Health Australia}

Australia has one of the best biosecurity systems in the World. Wildlife is part of that system. Wildlife Health Australia Incorporated (Wildlife Health Australia; WHA) is an initiative of the Australian government comprising a network of stakeholders across Australia with an interest in wildlife health. The core business activity is coordination of wildlife health surveillance information that can be used to improve decision making, management and policy development to protect Australia's natural environment, trade, human health, livestock health, biodiversity and tourism. Funding is primarily from the Australian Government Department of Agriculture and Water Resources (DAWR) through the Caring for Our Country (CFoC) program, with the understanding that, with other funding, WHA could also become more involved with biodiversity, human health and environmental issues. However, most of WHA's programs and activities function within a One Health space. This paper presents some of the activities that are working well and explains why. It discusses the future for wildlife health in Australia, opportunities for potential collaboration, and concludes that though much good work has been done, much is still to do and there is the need for sustained direction and focus.

\section{Building Strong One-Health Collaborations in Academia: Key Building Blocks}

Agnes Yawe ${ }^{1}$, Timothy Wakabi ${ }^{2}$, Irene Naigaga ${ }^{2}$ and William Bazeyo

${ }^{1}$ One Health Central and Eastern Africa

${ }^{2}$ OHCEA Secretariat

Increasing disease burdens with human-animal-ecosystem interface calls for robust approaches to deal with intertwined nature of diseases. While one-health approach gained prominence globally, the art of multi-disciplinary collaboration 
among health professionals is still a challenge with minimal efforts during outbreak response. Successful multi-disciplinary collaborations are needed now than ever to handle recurrent disease burdens. This abstract is about a successful one-health collaboration in higher institutions of learning "One Health Central and Eastern Africa (OHCEA)" working across human and animal health disciplines in diverse geographic areas. It enumerates key building blocks behind OHCEA model focusing on "soft elements". OHCEA is a mega coalition model going by Canadian Coalition for Global Health Research. It is a complex collaboration involving southern, northern institutions of learning working to re-construct learning and teaching at universities. Key building blocks for its success are: strong belief in common goal as a shared responsibility by leaders; mutual trust among individuals behind the initiative; unwavering support and conviction from sponsors of the pivotal positioning of universities in producing new cadre of health professionals; all backed by strong personal conviction and effort by founder member(s) in making the complex collaboration a success.

\title{
251
}

\section{Importance of Ecological Education for Anti-dengue Efforts: An Outreach Exercise at Japanese Primary Schools in Singapore}

\author{
Minako Jen Yoshikawa ${ }^{1}$, Christina Liew ${ }^{2}$, Shinji Fukushima ${ }^{3}$, Yuki Tada ${ }^{3}$ and Atsuo Hamada ${ }^{3}$ \\ ${ }^{1}$ Kyoto University \\ ${ }^{2}$ Environmental Health Institute, National Environment Agency \\ ${ }^{3}$ Travellers' Medical Centre, Tokyo Medical University Hospital
}

Mosquito-borne dengue virus infection is an increasing public health risk of global concern. Contributory factors include global warming, people movement, urbanisation, and the diminishing effectiveness of traditional vector control methods. Despite a well-established national dengue control programme, including comprehensive mosquito control and rigorous public health education conducted in all four official languages, the disease is endemic in Singapore-a vibrant business hub and tourist destination. Our recent investigation amongst a segment of the Japanese residents in Singapore, have revealed insufficiency in both knowledge of the disease and frequency of preventative actions taken. It is possible that the abundant information disseminated by the Singapore government has neither reached this demographic, nor been properly understood. We thus conducted outreach efforts in January 2016, involving 1,105 Japanese schoolchildren, to highlight the important linkage between ecology and public health. Our ecological education included the life cycle of mosquitoes in relation to the environment, and the effects of environmental parameters and climate change, employing talks, posters, videos and live mosquito exhibits. Such education is imperative for raising environmental awareness, and encouraging schoolchildren to become advocates of anti-dengue measures, to thus share the importance of source reduction and the role that each individual can play.

\section{8}

\section{Improving Food Security in Africa by Enhancing Resistance to Newcastle Disease and Heat Stress in Chickens: Genomics to Improve Poultry}

Huaijun Zhou ${ }^{1}$, David Bunn ${ }^{1}$, Rodrigo Gallardo ${ }^{1}$, Susan Lamont ${ }^{2}$, Jack Dekkers ${ }^{2}$, Amandus Muhairwa ${ }^{3}$, Peter Msoffe $^{3}$, Boniface Kayang ${ }^{4}$, Augustine Naazie ${ }^{4}$, George Aning ${ }^{4}$, Carl Schmidt ${ }^{5}$ and Terra Kelly

${ }^{1}$ University of California, Davis

${ }^{2}$ Iowa State University

${ }^{3}$ Sokoine University of Agriculture

${ }^{4}$ University of Ghana

${ }^{5}$ University of Delaware 
Homestead and small-scale poultry production has tremendous potential for alleviation of malnutrition and poverty in climate-stressed rural communities in Africa. Poor animal health and husbandry practices limit village poultry production throughout Africa. Newcastle disease (ND) is the number one constraint of raising poultry in Africa, causing mortality as high as $80 \%$ among village flocks. The goal of this USAID-supported program is to utilize deep phenotyping combined with advanced genomic selection to sustainably enhance innate resistance to ND and heat stress in indigenous African chickens to improve production. Genes and signal pathways associated with genetic resistance to NDV infection and heat stress were identified by RNA sequencing in well-established ND-resistant and susceptible inbred lines in the US by challenging chickens with a lentogenic ND virus strain in temperate or hot environments. Six African chicken ecotypes and one commercial layer line widely used in Africa were challenged. The chicken $600 \mathrm{~K}$ SNP chip was used to identify SNP or regions associated with resistance to NDV and heat stress. Enhanced resistant birds will be selected and bred for distribution to smallholder farmers and households. The expected results will help achieve the USAID Feed the Future Program goals to reduce hunger and strengthen food security.

1011

\section{Bats and Bushmeat: Targeting High Risk Taxa and Behaviours for Prevention of Viral Spillover}

Sijali Zikankuba ${ }^{1}$, Jonna Mazet ${ }^{2}$, David Wolking ${ }^{2}$, Elizabeth VanWormer ${ }^{3}$, Christopher Kilonzo ${ }^{2}$, Tracey Goldstein $^{2}$ and Rudovick Kazwala

${ }^{1}$ Sokoine University of Agriculture, Morogoro, Tanzania and Health for Animals and Livelihood Improvement (HALI) Project, Tanzania

${ }^{2}$ One Health Institute, University of California Davis, Davis, California and Health for Animals and Livelihood Improvement (HALI) Project, Tanzania

${ }^{3}$ Health for Animals and Livelihood Improvement (HALI) Project, Tanzania and School of Veterinary Medicine and Biomedical Sciences, School of Natural Resources, University of Nebraska-Lincoln, Lincoln, Nebraska, USA

Climate change and human population growth have the potential to influence the distribution and population dynamics of bats and other wild animals leading to increased human-wildlife interactions. The launch of the PREDICT project in 2009 in Tanzania improved awareness of the presence of high-risk human-animal interfaces, resulted in targeted wildlife sampling of key taxa at prioritized sites representative of Tanzania's biological and geographical diversity and has helped to improve the infrastructure and human resource base for the detection of emerging viruses of pandemic potential. More than 64 viruses were detected in 268 animals (bats, rodents and non-human primates), and 75\% of these viruses were from bat hosts. In addition to investigating connections between people and key taxa like bats that harbor diverse viruses, we also focused on the viral transmission risks associated with common routes of contact with diverse wildlife taxa, like hunting and examined practices associated with bushmeat hunting, targeting villages bordering the Ruaha ecosystem. Ethnographic data collected from community members revealed risky practices associated with hunting and meat preparation and consumption. Results also indicated that the communities had a limited awareness of the potential risk for zoonotic disease transmission associated with wildlife hunting and consumption. 


\section{Determinants of the Effectiveness of Mass Dog Vaccination to Eliminate Rabies in African Cities}

Jakob Zinsstag, Monique Léchenne, Mirjam Laager, Rolande Mindekem, Service Naïssengar, Assandi Oussigéré, Kebkiba Bidjeh, Germain Rives, Julie Teissier, Daugla D. Moto, Idriss O. Alfaroukh, Yvonne Muthiani, Abdallah Traoré, Jan Hattendorf, Anthony Lepelltier, Hervé Bourhy, Laurent Dacheux, Tanja Stadler and Nakul Chitnis Swiss Tropical and Public Health Institute, University of Basel

After polio, dog transmitted rabies is one of the most promising viral diseases to be targeted for elimination. Two consecutive dog mass vaccination campaigns in 2012 and 2013, which were co-funded by the Chadian government and external donors, were sufficient to interrupt transmission for more than two years in N'Djamena, Chad. A deterministic dog-human rabies transmission model, fitted to routine weekly data on rabid dogs and exposed human cases demonstrated the elimination, and it was confirmed by a phylo-dynamic estimation of the reproductive number from dog related rabies virus (RAV) genetic sequences. Similar small scale mass vaccinations in Bamako, Mali did not reach sufficient coverage to interrupt transmission. The low coverage in Bamako was assessed by a unique mixed method effectiveness model showing that the lack of information in households was a main factor for the poor vaccination coverage in the study area. These results show that dog rabies can be eliminated in African cities with currently-available dog vaccines provided that the communities are well informed and engaged. 